\title{
Decarboxylative Alkyl Coupling Promoted by NADH and Blue Light
}

Rajdip Chowdhury ${ }^{\ddagger}$, Zhunzhun $\mathrm{Yu}^{\ddagger}$, My Linh Tong, Stefanie V. Kohlhepp, Xiang Yin, and Abraham Mendoza*

Dept. of Organic Chemistry, Arrhenius laboratory, Stockholm University, 10691 Stockholm (Sweden)

‡These authors contributed equally

Corresponding author email: abraham.mendoza@su.se

Raw data for this article can be downloaded from Zenodo https://dx.doi.org/10.5281/zenodo.4106400 


\section{Table of Contents}

1. General Information 1

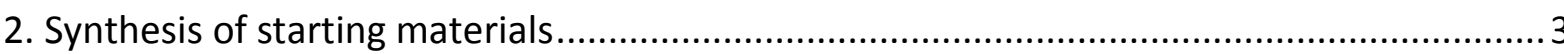

General procedure (A1): Synthesis of redox-active esters from solid carboxylic acids......... 3

General procedure (A2): Synthesis of redox-active esters from liquid carboxylic acids ....... 3

Synthesis of 1,3-dioxoisoindolin-2-yl (1R,4R)-bicyclo[2.2.1] hept-5-ene-2-carboxylate (2q) ..........5

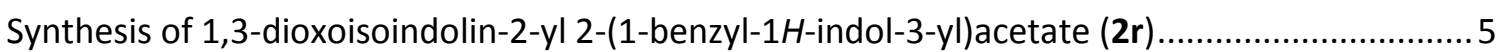

Synthesis of 1,3-dioxoisoindolin-2-yl (1R,4R)-7,7-dimethyl-2-oxobicyclo[2.2.1]heptane-1-

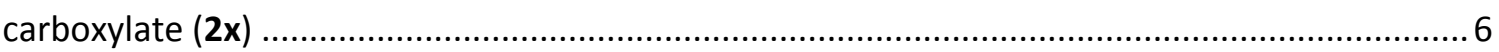

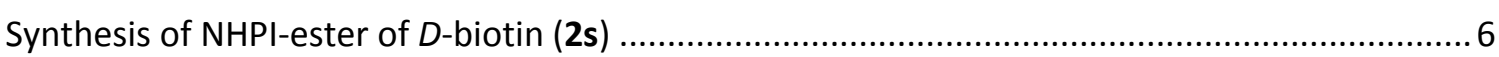

Synthesis of NHPI ester of derivative of 186-Glycyrrhetinic acid (2aa) ........................................ 7

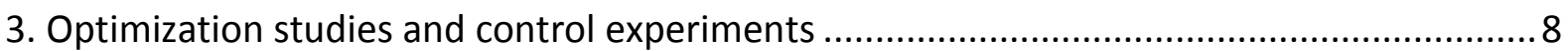

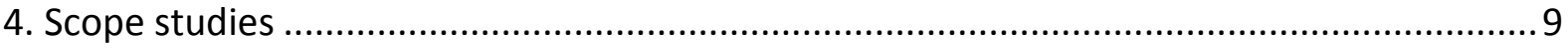

General Procedure (B1): Decarboxylative coupling of redox-active carboxylates with electron-poor olefins using BuNAH (10) and blue light ................................................. 9

General Procedure (B2): Decarboxylative coupling of redox-active carboxylates with electron-poor olefins using BuNAH (10) and blue light NADH (11) and blue ..................... 10

General procedure (B3): Preparative decarboxylative coupling of redox-active carboxylates with electron-poor olefins BuNAH (10) and blue light. ............................... 10

Synthesis of benzyl 3-(1-methylcyclohexyl)propanoate (4a).......................................................11

Synthesis of methyl 3-(1-methylcyclohexyl)propanoate (4b).................................................. 11

Synthesis of N,N-dimethyl-3-(1-methylcyclohexyl)propanamide (4c)........................................ 12

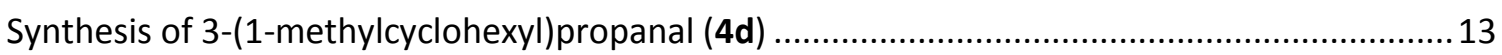

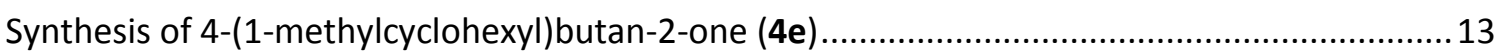

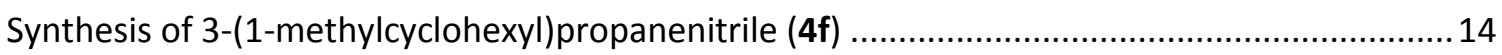

Synthesis of ((2-(1-methylcyclohexyl)ethyl)sulfonyl)benzene (4g) ............................................ 14

Synthesis of 3-(2-(1-methylcyclohexyl)ethyl)pyrrolidine-2,5-dione (4h) ..................................... 15

Synthesis of 1-pentyl-4-(2-(phenylsulfonyl)ethyl)bicyclo[2.2.2]octane (4i) ................................ 16

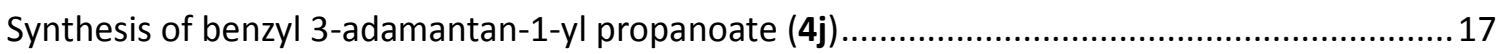

Synthesis of tert-butyl 4-(3-(benzyloxy)-3-oxopropyl)-4-methylpiperidine-1-carboxylate (4k) ... 17

Synthesis of ((2-(1-phenylcyclopropyl)ethyl)sulfonyl)benzene (4I) ............................................ 18

Synthesis of benzyl 7-(2,5-dimethylphenoxy)-4,4-dimethylheptanoate (4m) ........................... 19

Synthesis of 1-(7-(2,5-dimethylphenoxy)-4,4-dimethylheptanoyl)piperidine-4-carboxylic acid (4n)

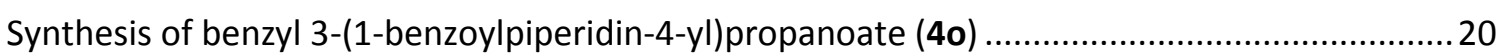


Synthesis of 2-(2-(phenylsulfonyl)ethyl)-2,3-dihydro-1H-indene (4p)

Synthesis of $(1 S, 4 S)$-5-(2-(phenylsulfonyl)ethyl)bicyclo[2.2.1]hept-2-ene (4q) and 3-(2-

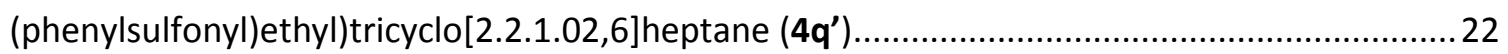

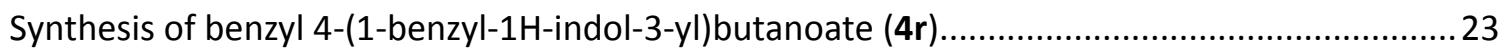

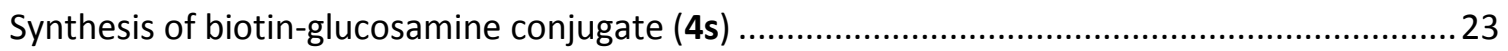

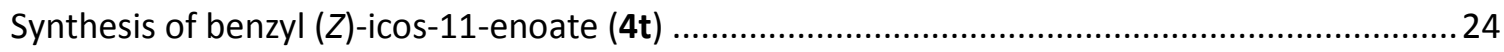

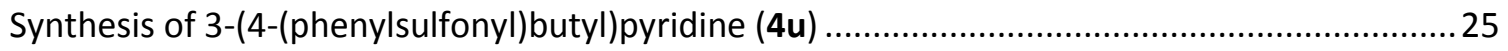

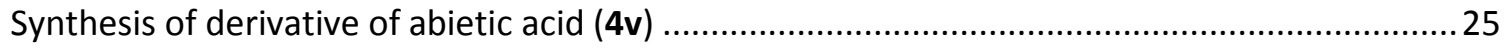

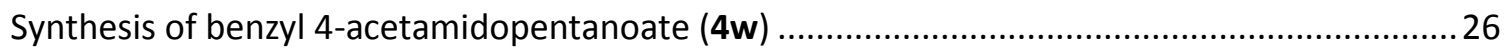

Synthesis of benzyl 3-((1R,4R)-7,7-dimethyl-2-oxobicyclo[2.2.1] heptan-1-yl)propanoate (4x) ...27

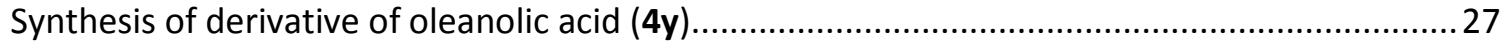

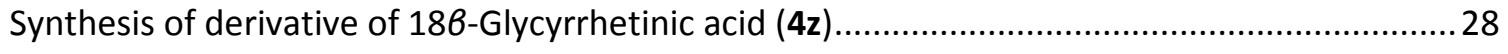

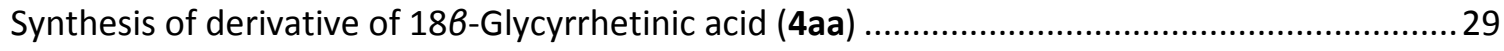

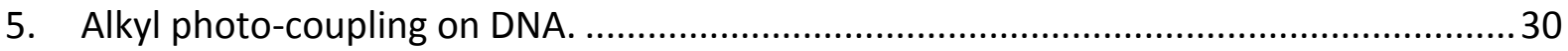

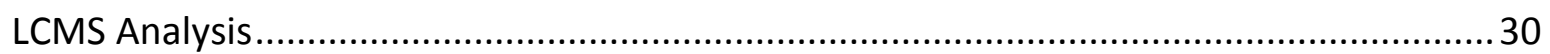

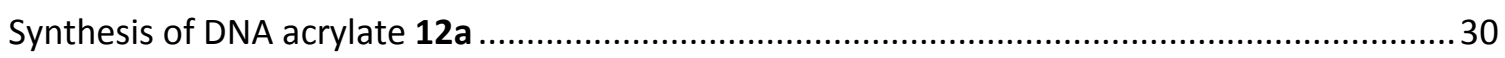

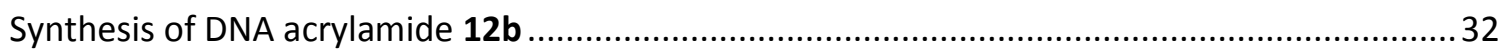

General procedure (C): Photo-mediated decarboxylative coupling of redox-active esters

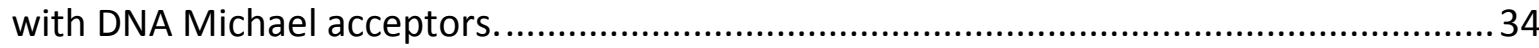

Yield determination of the photo mediated decarboxylative coupling reaction of DNA

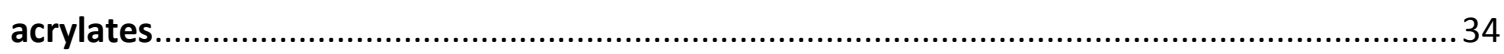

Synthesis of DNA functionalized product 13aa using BuNAH (10) as a reductant ......................... 35

Synthesis of DNA functionalized product 13aa using NADH (11) as a reductant ..........................36

Synthesis of DNA functionalized product 13ab using BuNAH (10) as a reductant ...........................

Synthesis of DNA functionalized product 13ab using NADH (11) as a reductant...........................38

Synthesis of DNA functionalized product 13ac using BuNAH (10) as a reductant ........................39

Synthesis of DNA functionalized product 13ac using NADH (11) as a reductant ...........................40

Synthesis of DNA functionalized product 13ad using BuNAH (10) as a reductant ........................4 41

Synthesis of DNA functionalized product 13ad using NADH (11) as a reductant.........................42

Synthesis of DNA functionalized product 13ae using BuNAH (10) as a reductant ....................... 43

Synthesis of DNA functionalized product 13ad using NADH (11) as a reductant......................... 44

Synthesis of DNA functionalized product 13ba using BuNAH (10) as a reductant ........................45

Synthesis of DNA functionalized product 13ba using NADH (11) as a reductant ..........................46

Synthesis of DNA functionalized product 13bb using BuNAH (10) as a reductant........................47

Synthesis of DNA functionalized product 13bb using NADH (11) as a reductant......................... 48

Synthesis of DNA functionalized product 13bc using BuNAH (10) as a reductant .......................49 
Synthesis of DNA functionalized product 13 bc using NADH (11) as a reductant ........................50

Synthesis of DNA functionalized product 13be using BuNAH (10) as a reductant ........................53

Synthesis of DNA functionalized product 13be using NADH (11) as a reductant.........................54

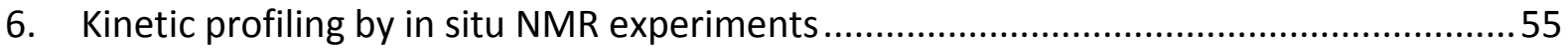

Procedure for the in situ NMR experiment under standard reaction conditions (no-D

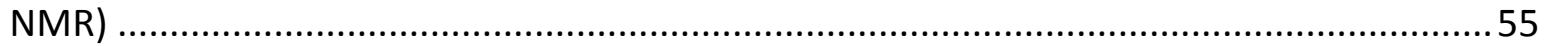

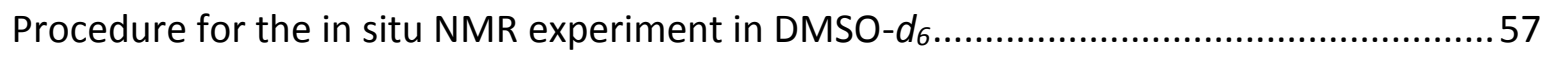

Procedure for the in situ NMR experiment to profile the background and deactivation of

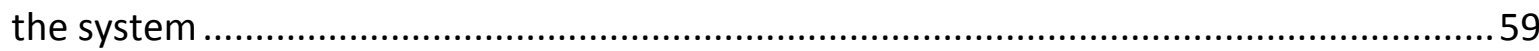

Procedure for the in situ NMR experiment to study the kinetic effect of the initial

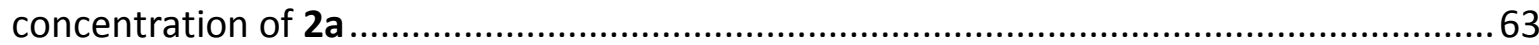

Procedure for the control reaction for the inner filter effect.........................................65

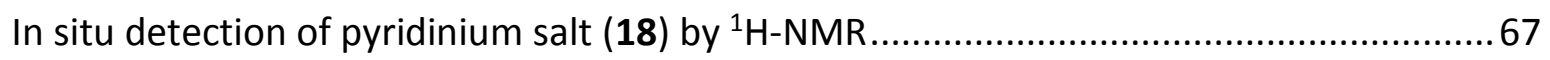

7. Preliminary evaluation of the photo-coupling with NADH (11) ......................................69

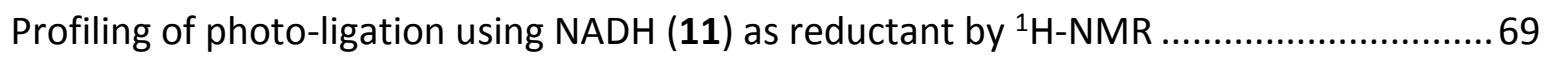

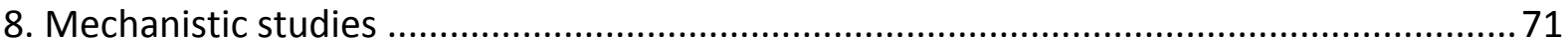

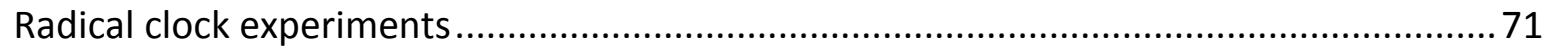

Syntheses of benzyl 4-cyclopentylbutanoate (4ab) and benzyl non-8-enoate (4ab') ..................71

Characterization of benzyl 4-cyclopentylbutanoate (4ab) ........................................................ 71

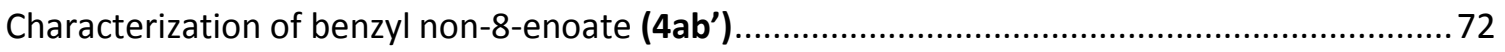

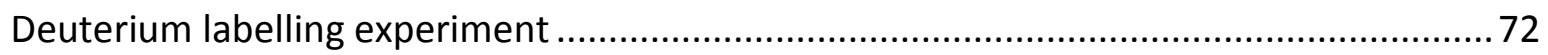

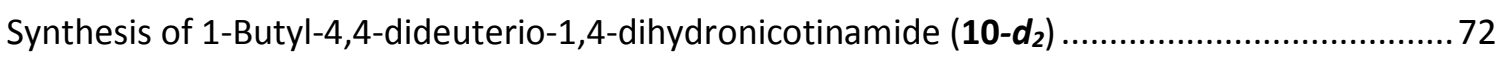

Determination of the quantum yield of the photo-ligation .......................................... 73

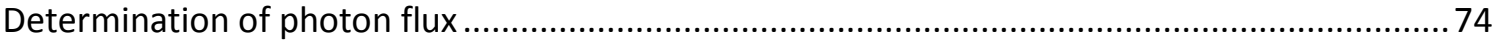

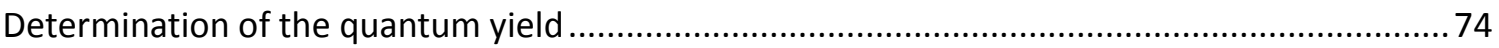

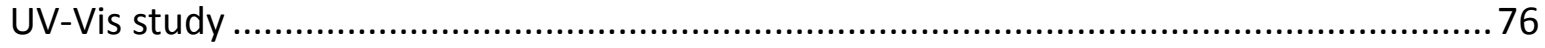

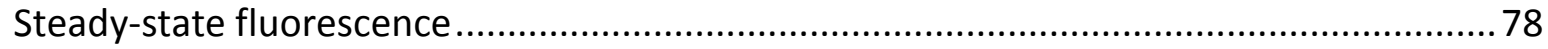

Emission spectrum of BuNAH (10) and BuNAH (10) - redox-active ester 2a mixture ...................78

Excitation spectrum of BuNAH (10) and BuNAH (10) - redox-active ester 2a mixture.................79

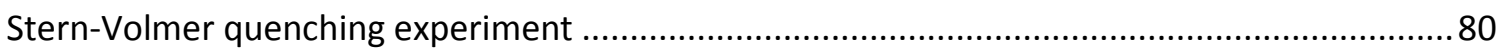

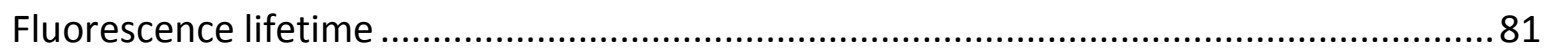

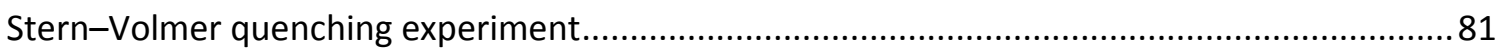

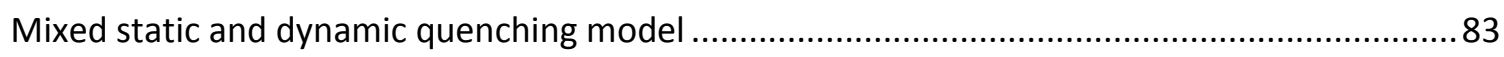

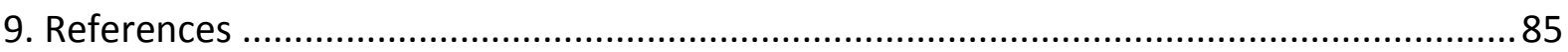


10. NMR spectra of synthesised compounds .86

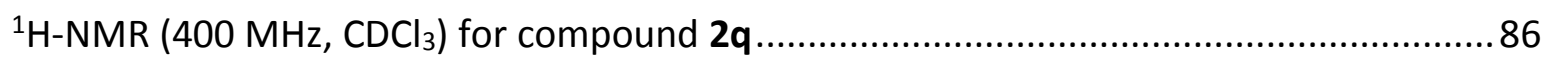

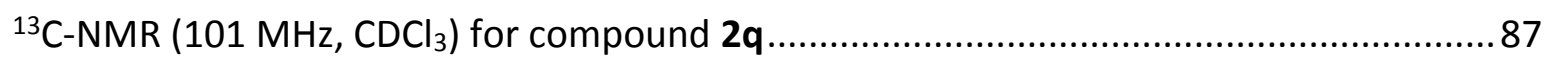

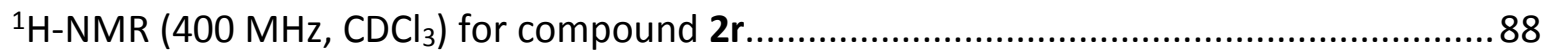

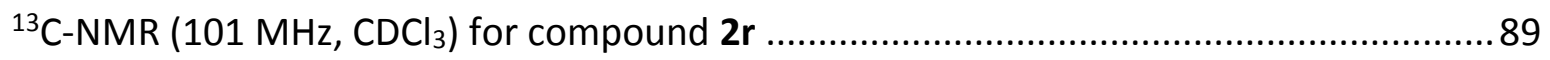

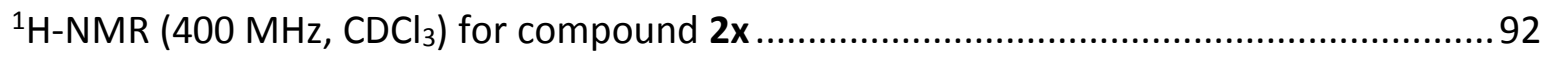

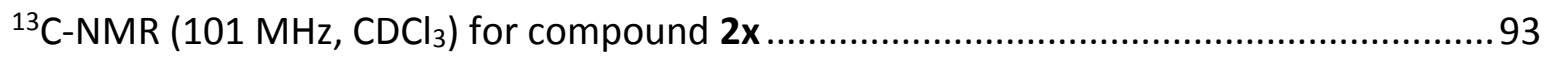

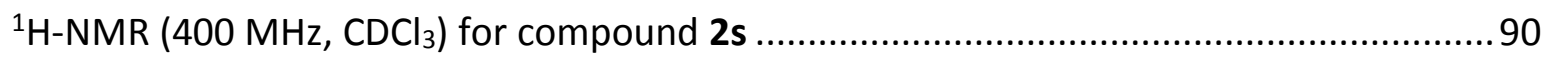

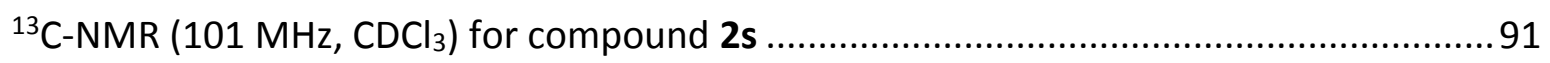

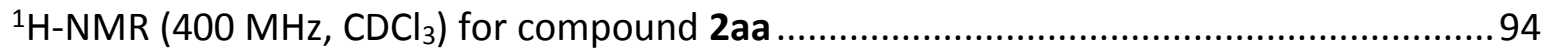

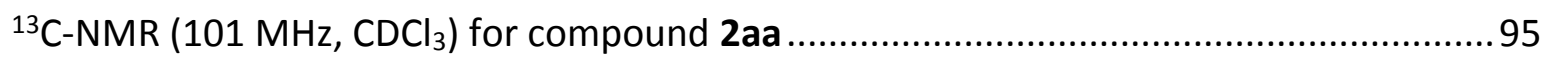

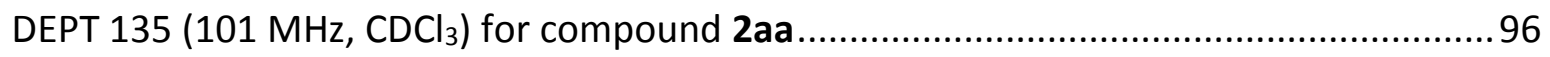

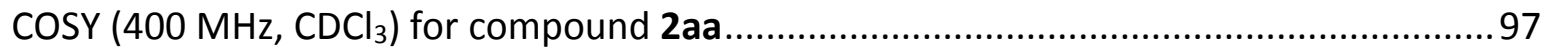

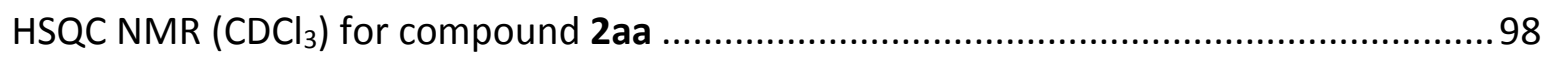

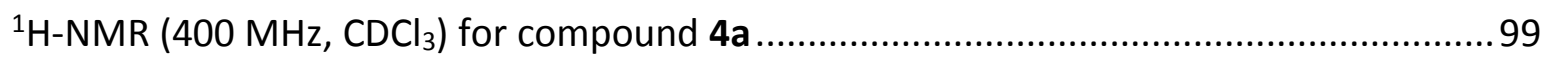

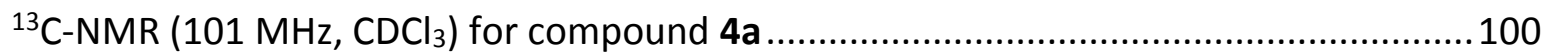

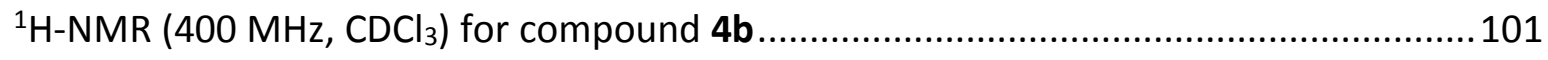

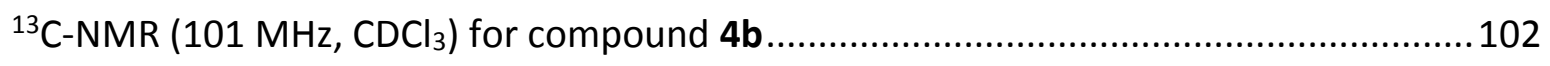

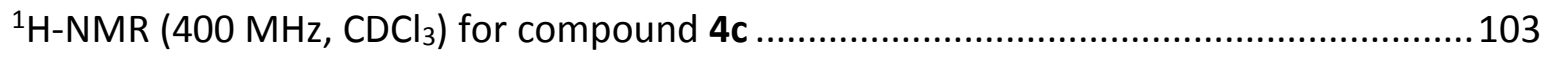

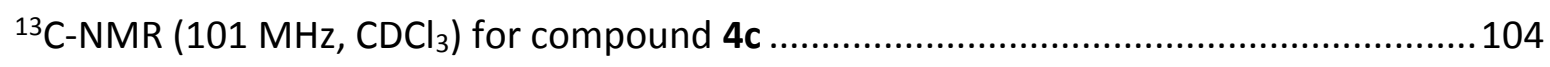

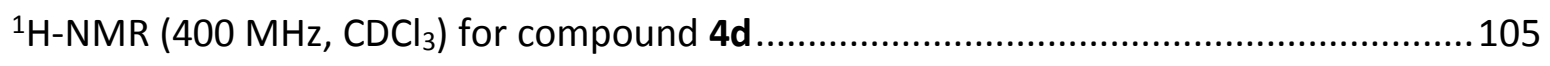

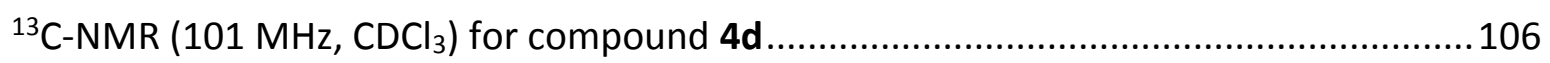

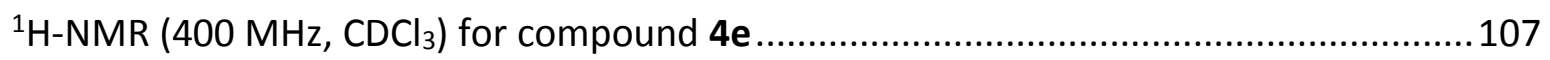

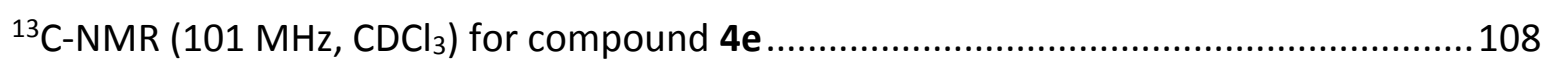

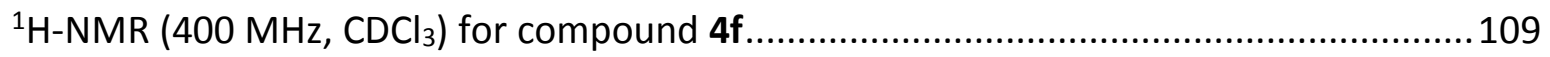

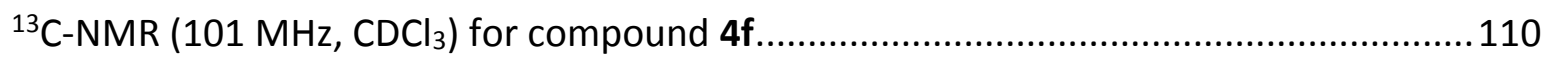

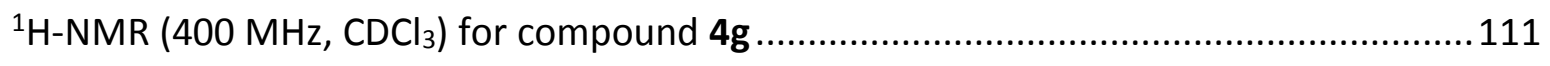

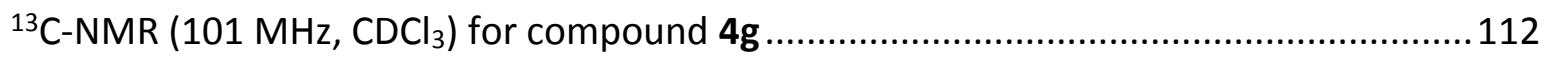

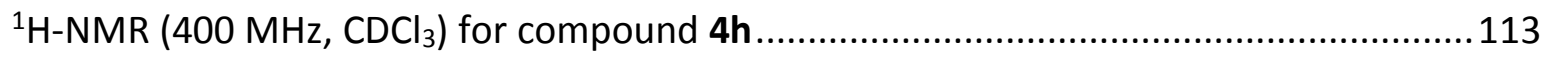

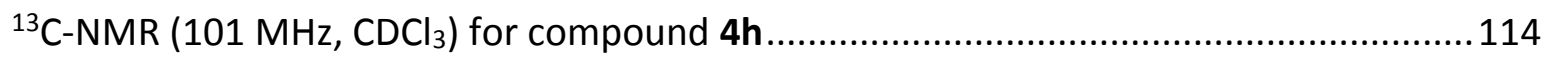

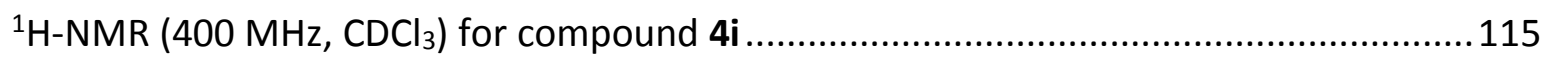

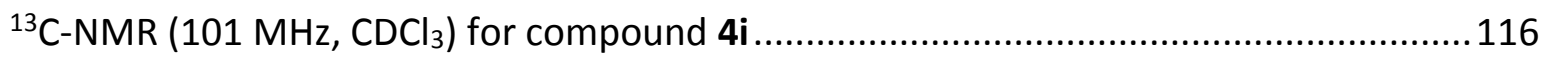


${ }^{1} \mathrm{H}-\mathrm{NMR}\left(400 \mathrm{MHz}, \mathrm{CDCl}_{3}\right)$ for compound $\mathbf{4 j}$ 117

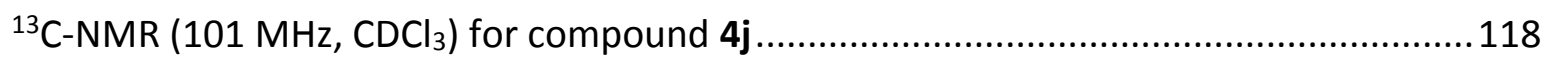

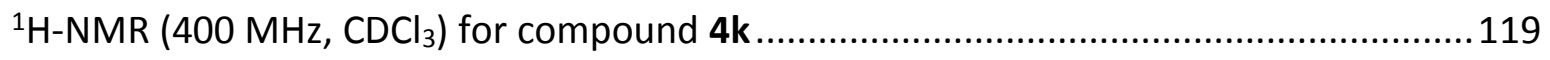

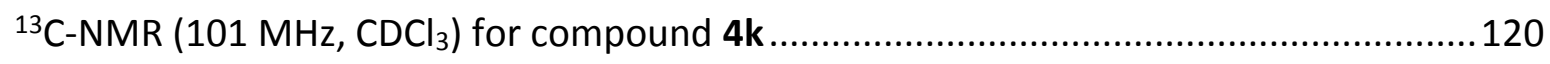

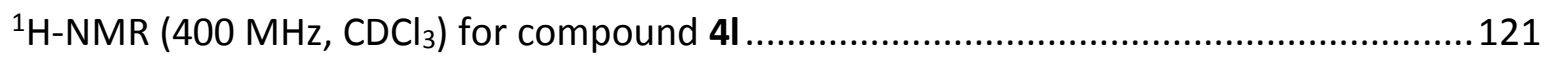

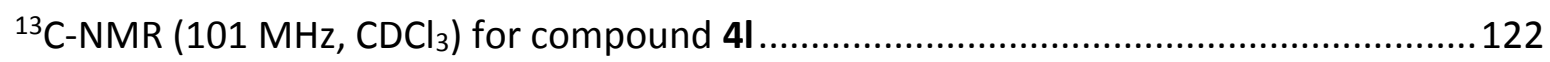

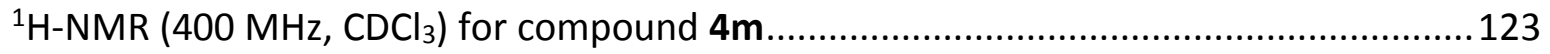

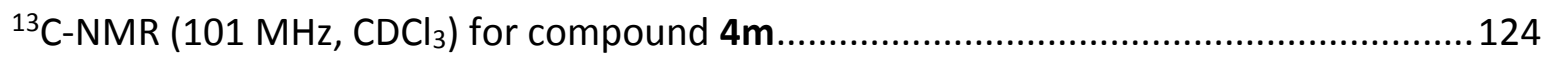

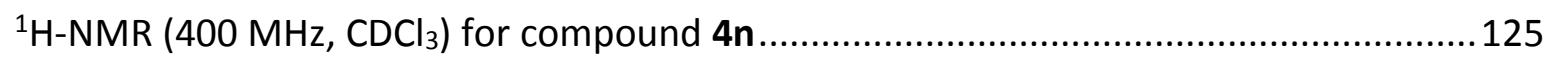

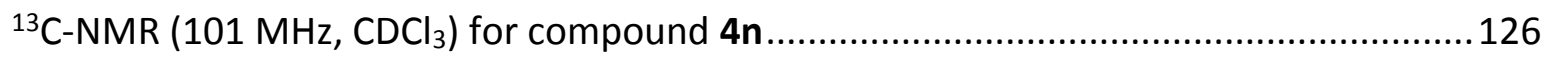

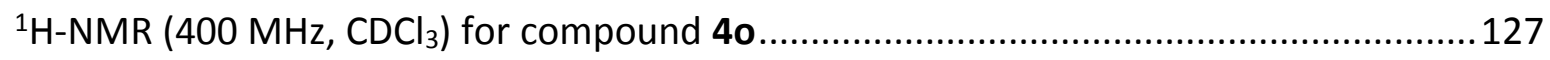

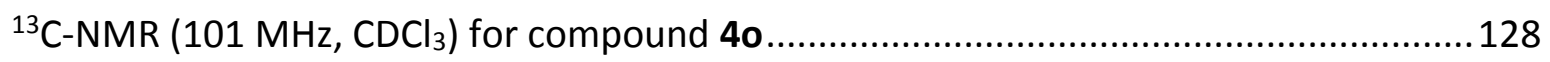

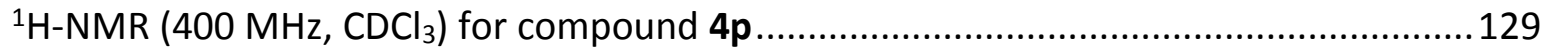

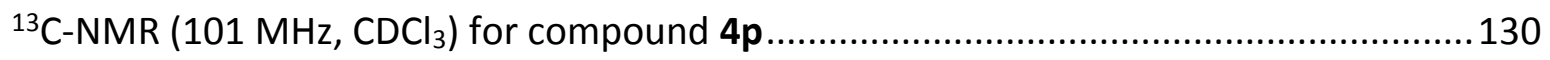

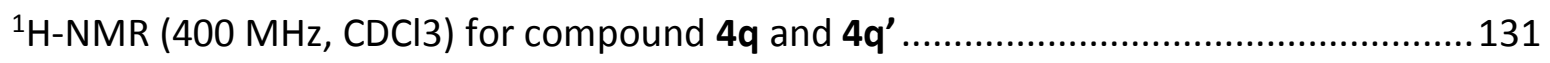

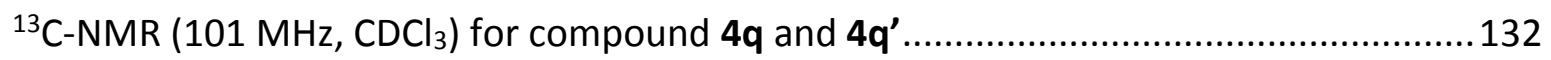

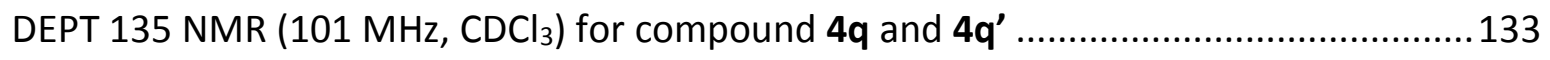

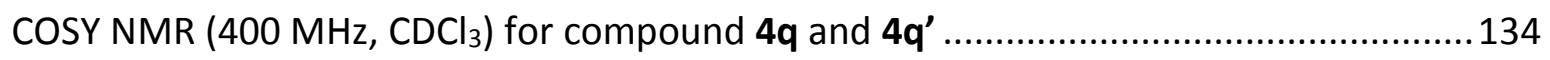

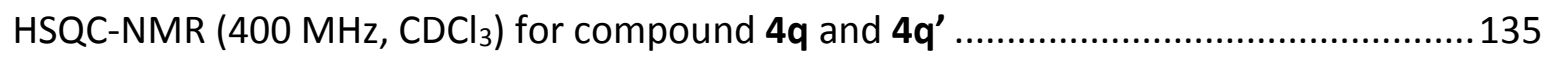

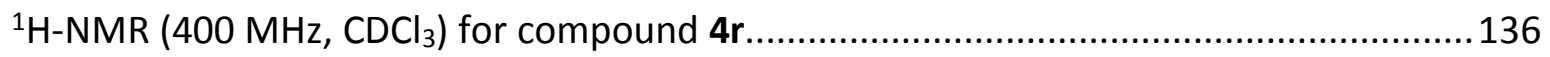

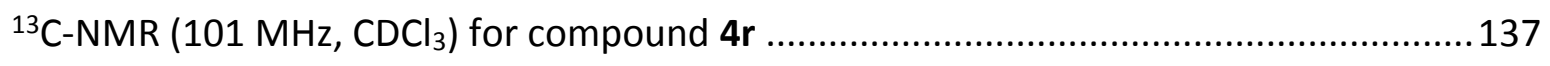

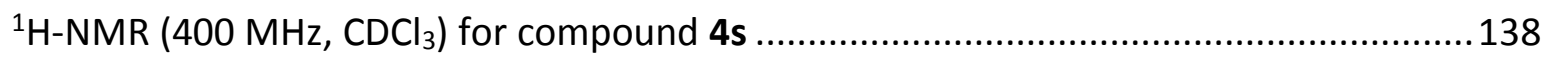

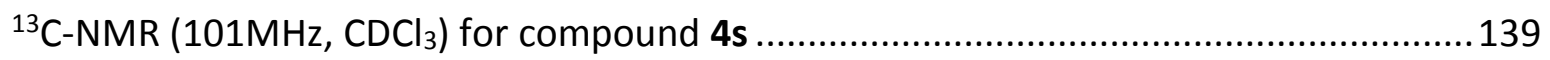

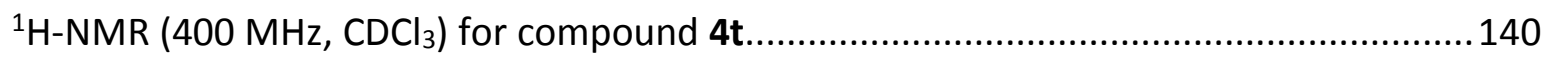

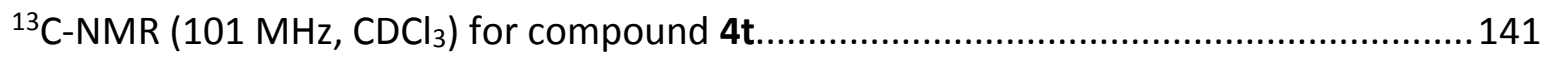

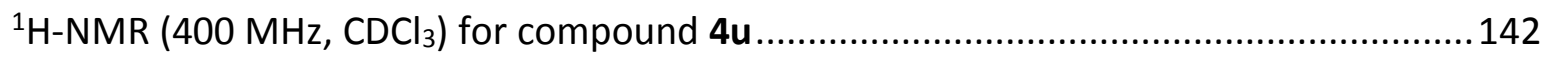

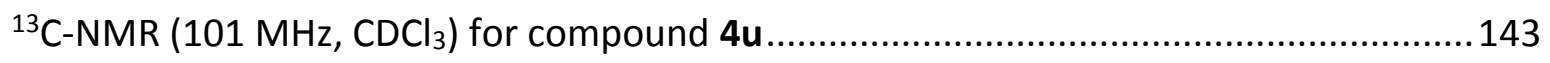

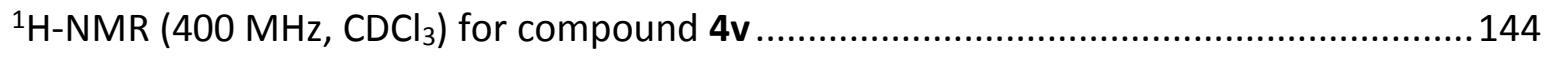

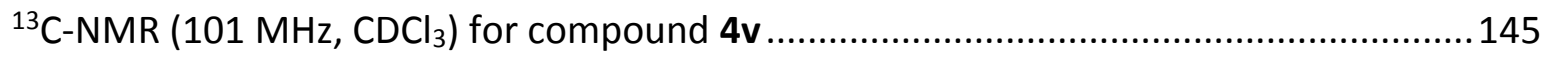

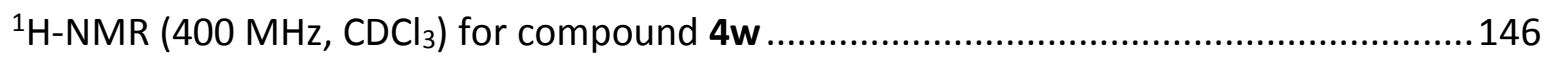

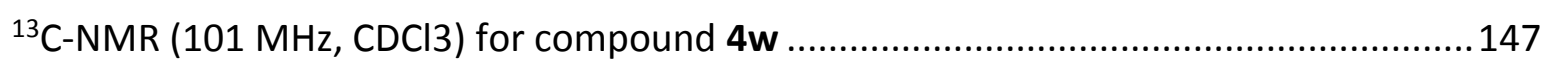

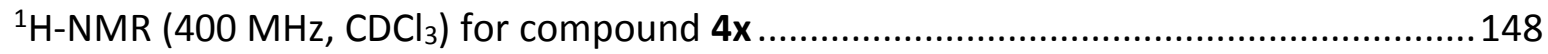




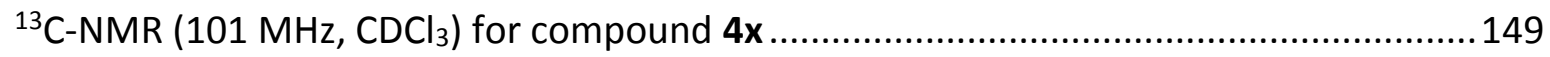

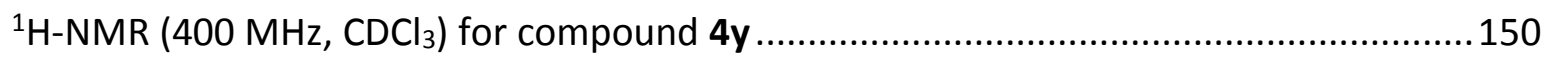

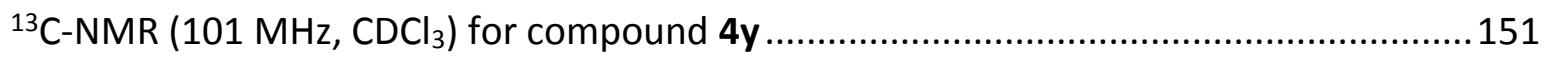

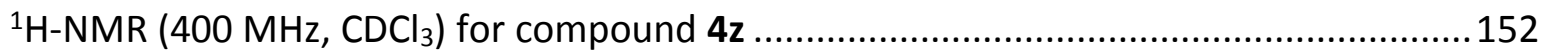

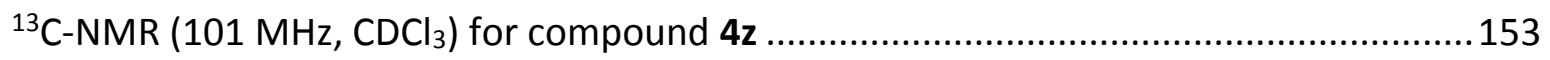

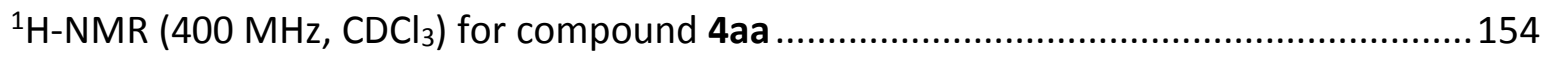

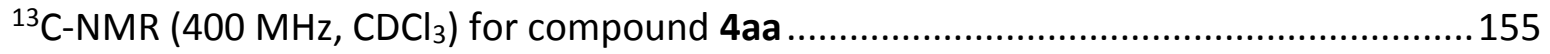

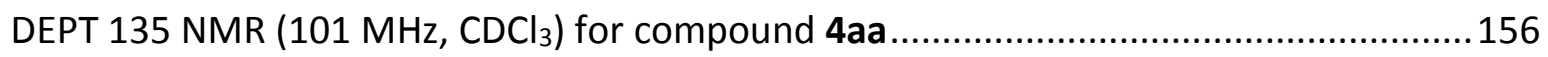

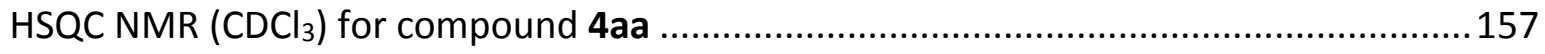

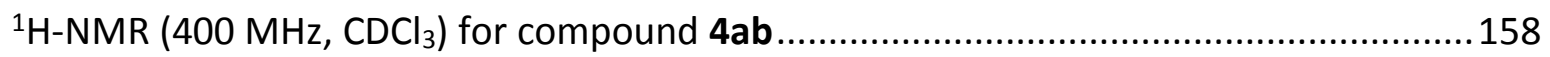

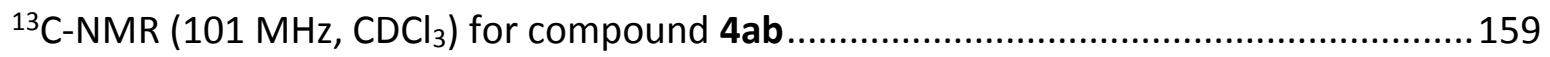

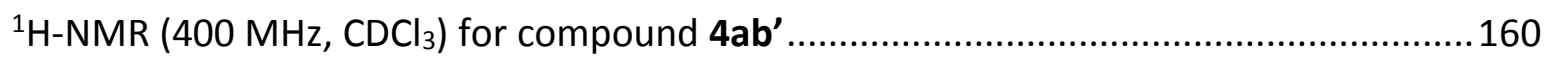

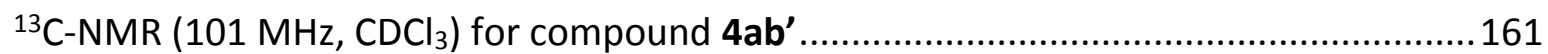

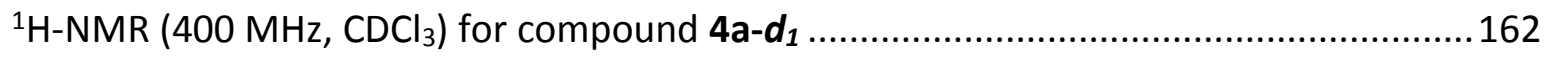

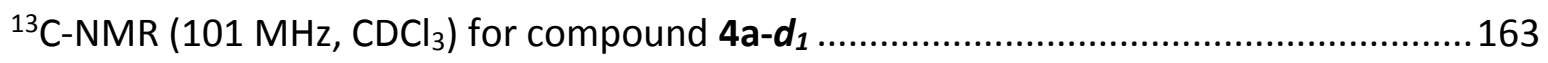




\section{General Information}

Materials: All reactions were carried out using oven-dried glassware under an atmosphere of argon unless otherwise specified. Carboxylic acids were purchased from Sigma Aldrich, Fluorochem and TCl Chemicals. Dry solvents were obtained by passing them through activated alumina columns. Anhydrous dimethyl sulfoxide was purchased from Sigma Aldrich. The solvents used in column chromatography, petroleum ether, pentane, dichloromethane, methanol and ethyl acetate, were obtained from commercial suppliers in HPLC grade and used without further purification. Dihydropyridine was synthesized using the literature procedure reported by Hollmann and co-workers. ${ }^{[12]}$ S2-DNA headpiece $\left(5^{\prime} \mathrm{d}\right.$ Phos-GAGTCASpacer 9-Amino C7-Spacer 9-TGACTCCC 3') was purchased from LGC Biosearch Technologies.

Chromatography: Thin layer chromatography (TLC) was carried out on $0.25 \mathrm{~mm}$ E. Merck silica plates $\left(60 \mathrm{~F}_{254}\right)$ using UV light $(\lambda=254 \mathrm{~nm})$ as visualizing agent and a vanillin, phosphomolybdic acid (PMA) or $\mathrm{KMnO}_{4}$ solution and heat as developing agents, as specified. Flash column chromatography on $\mathrm{SiO}_{2}$ was performed using E. Merck silica gel $(60 \AA$, particle size $0.043-0.063 \mathrm{~mm}$ ).

Spectroscopy: NMR spectra for the characterization of compounds were recorded at room temperature on a Bruker instrument $400 \mathrm{MHz}\left({ }^{1} \mathrm{H}\right)$ and at $101 \mathrm{MHz}\left({ }^{13} \mathrm{C}\right)$ and $376 \mathrm{MHz}\left({ }^{19} \mathrm{~F}\right)$, or $500 \mathrm{MHz}\left({ }^{1} \mathrm{H}\right)$ and at $126 \mathrm{MHz}\left({ }^{13} \mathrm{C}\right)$. Chemical shifts $(\delta)$ are reported in ppm, using the residual solvent peak in $\mathrm{CDCl}_{3}\left(\delta_{\mathrm{H}}=7.26\right.$ and $\left.\delta_{\mathrm{C}}=77.16 \mathrm{ppm}\right)$, coupling constants $(J)$ are given in hertz $(\mathrm{Hz})$. Data are reported as follows: chemical shift, multiplicity (s: singlet, d: doublet, $\mathrm{t}$ : triplet, q: quartet, br: broad, m: multiplet), coupling constants and integration. Highresolution mass spectra (HRMS) were determined with a Bruker Daltonics microTOF Mass Spectrometer using an ESI ion source. UV-visible experiments were done using a Cary-50 spectrometer in advanced mode (settings: average time (sec): 0.250; data interval (nm): 1.00; scan rate (nm/min): 240.00 ) at wavelengths $300-800 \mathrm{~nm}$.

Experimental details: Starting materials were synthesized using common Pyrex ${ }^{\circledR}$ round bottom flasks. Photo-induced reactions were carried out in $5 \mathrm{~mL}$ flat bottomed vials crimped on top with $20 \mathrm{~mm}$ Sil/PTFE Septa (Cronus, SMI-LabHut Ltd.). Reactions were illuminated at $1 \mathrm{~cm}$ distance from the bottom of the reaction vial with a single Osram OSLON SSL 80 LED operating at $700 \mathrm{~mA}$ current. Reaction temperature was maintained using an aluminium block connected to a cooling Huber Minichiller 300 OLÉ⿴囗十

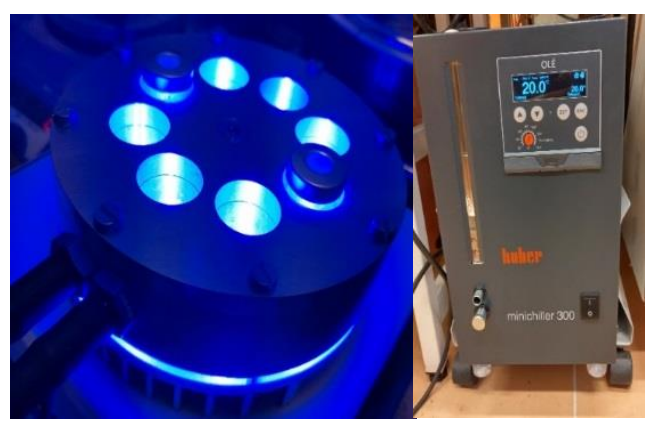

Figure S1: Experimental set up. 


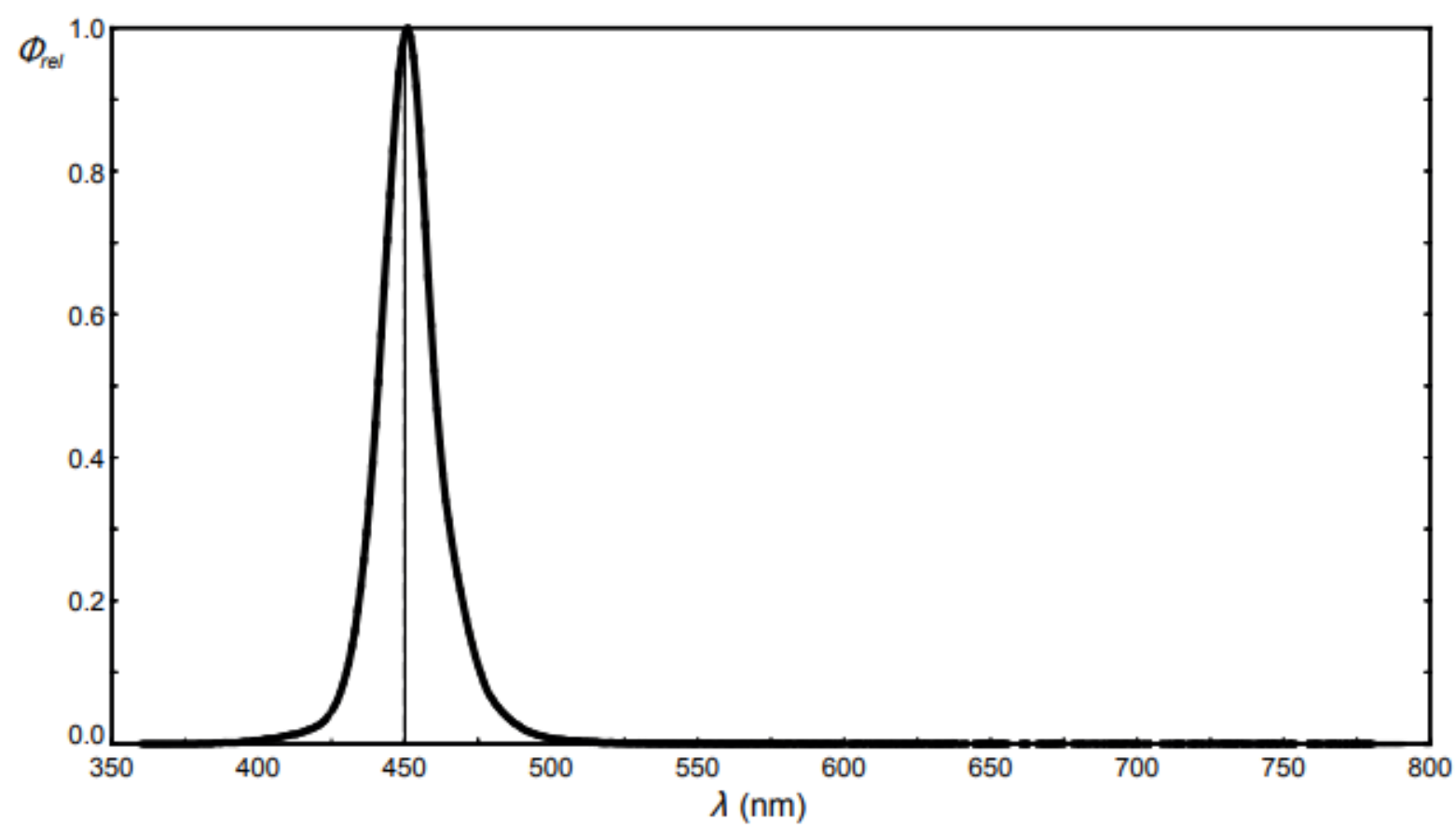

Figure S2: Normalized emission spectrum of the light source used in this study. 


\section{Synthesis of starting materials}

General procedure (A1): Synthesis of redox-active esters from solid carboxylic acids
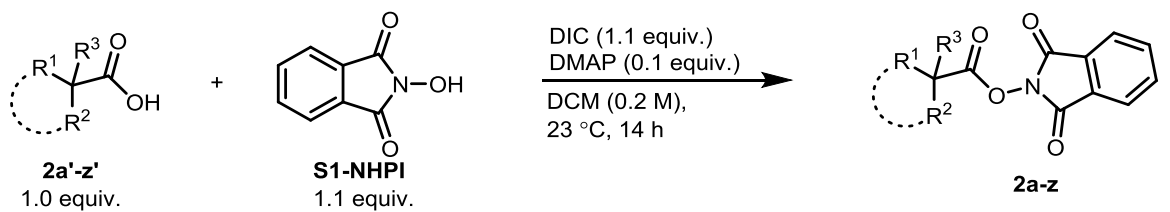

$N$-hydroxyphthalimide (S1; 1.1 equiv.), DMAP (0.1 equiv.) and carboxylic acid (solid) (1.0 equiv.) were charged in a round bottomed flask. DCM $(0.2 \mathrm{M})$ was added followed by dropwise addition of $N, N^{\prime}$-diisopropylcarbodiimide (1.1 equiv.). The resulted mixture was stirred overnight at room temperature. After completion of the reaction (as monitored by $\mathrm{TLC}$ ) the reaction mixture was filtered through a Celite pad $(1.5-2.0 \mathrm{~cm})$ and the solvent was removed under reduced pressure. The crude product was purified by column chromatography on $\mathrm{SiO}_{2}$ using a mixture of petroleum ether and EtOAc or DCM as the eluent.

General procedure (A2): Synthesis of redox-active esters from liquid carboxylic acids
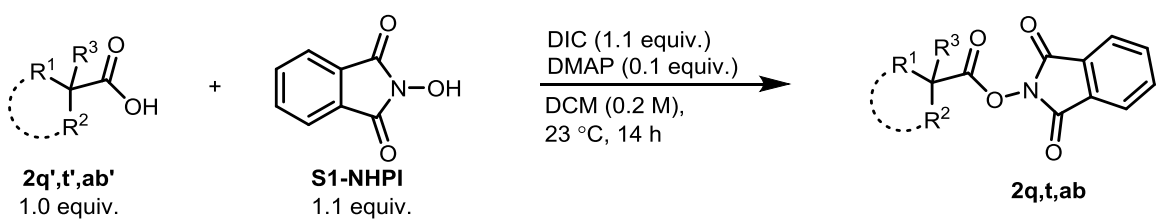

$N$-hydroxyphthalimide (S1; 1.1 equiv.) and DMAP (0.1 equiv.) were charged in a round bottomed flask. Carboxylic acid (1.0 equiv.) dissolved in DCM ( $0.2 \mathrm{M}$ ) was added followed by dropwise addition of $N, N^{\prime}$-diisopropylcarbodiimide (1.1 equiv.). The resulting mixture was stirred overnight at room temperature. After completion of the reaction (as monitored by TLC) the reaction mixture was filtered through a Celite pad $(1.5-2.0 \mathrm{~cm})$ and the solvent was removed under reduced pressure. The crude product was purified by column chromatography on $\mathrm{SiO}_{2}$ using a mixture of petroleum ether and EtOAc or DCM as the eluent. 
The following redox-active esters were synthesized according to the literature procedures. All the data are in accordance with the literature. ${ }^{[1-7]}$

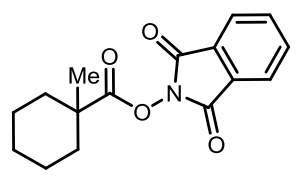

2a

synthesized by using procedure $\mathrm{A} 1$ ref. 2

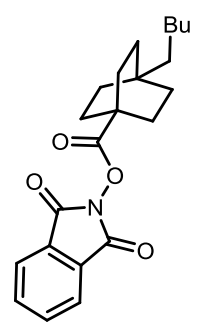

2i

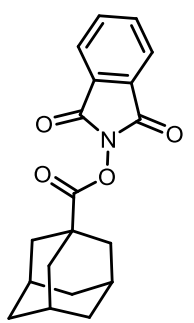

2j

synthesized by using procedure A1 ref. 2

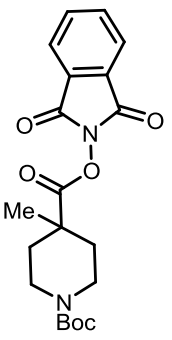

2k

synthesized by using procedure $\mathrm{A} 1$ ref. 5

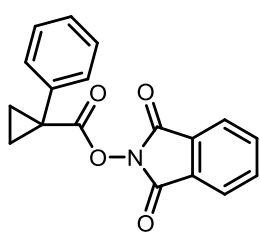

2I

synthesized by using procedure $\mathrm{A} 1$ ref. 1

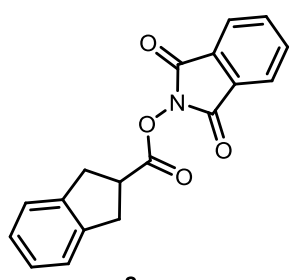

$2 p$

synthesized by using procedure A1 ref. 1

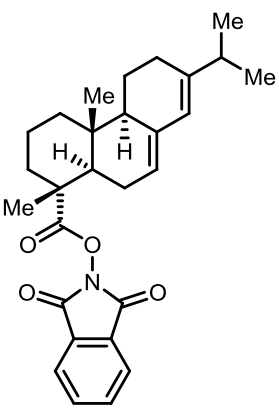

2v

synthesized by using

procedure A1 ref. 1

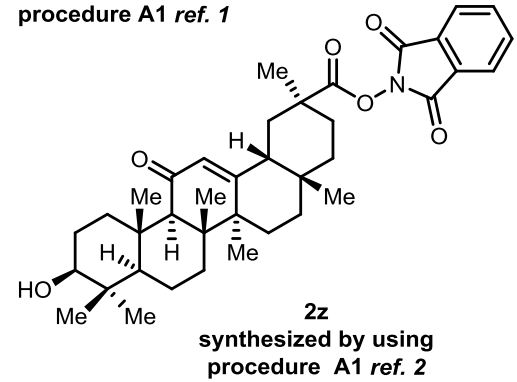

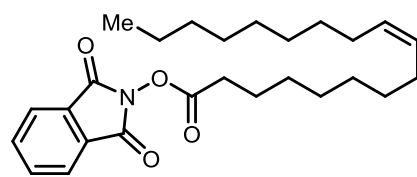

$2 t$

synthesized by using procedure A2 ref. 1

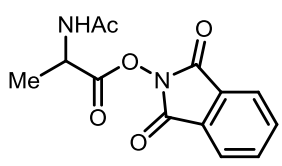

$2 w$

synthesized by using procedure $\mathrm{A} 1$ ref. 4<smiles>O=C(ON1C(=O)c2ccccc2C1=O)C1CCN(C(=O)c2ccccc2)CC1</smiles>

20

synthesized by using procedure A1 ref. 3

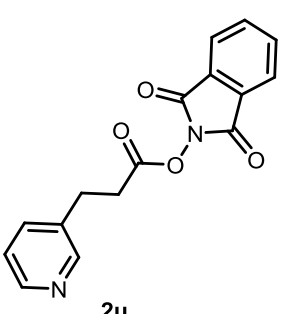

synthesized by using procedure A1 ref. 1

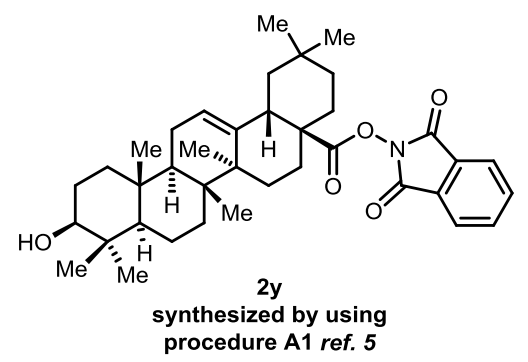

Figure S3: Reported NHPI-esters.

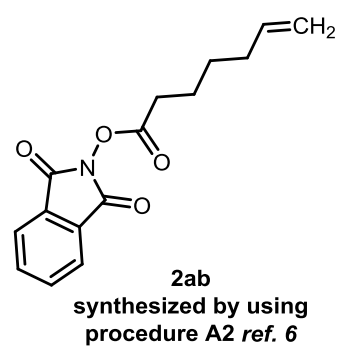


Synthesis of 1,3-dioxoisoindolin-2-yl (1R,4R)-bicyclo[2.2.1] hept-5-ene-2-carboxylate (2q)
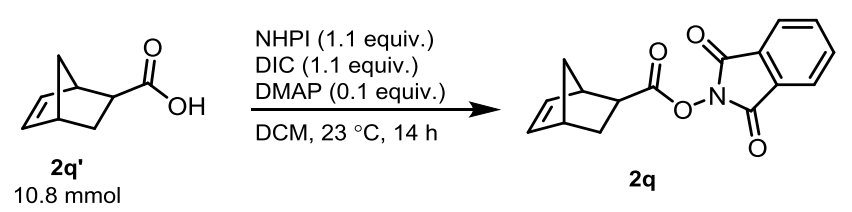

General procedure A2 was applied using $(1 R, 4 R)$-bicyclo[2.2.1] hept-5-ene-2-carboxylic acid (98\% endo) (2q; $1.5 \mathrm{~g}, 10.8 \mathrm{mmol}, 1.0$ equiv.), $N$-hydroxyphthalimide (S1; $1.9 \mathrm{~g}, 11.9 \mathrm{mmol}$, 1.1 equiv.), $N, N^{\prime}$-diisopropylcarbodiimide $(1.5 \mathrm{~g}, 11.9 \mathrm{mmol}, 1.1$ equiv.) and DMAP (133 mg, $1.1 \mathrm{mmol}, 0.1$ equiv.) in $50 \mathrm{~mL} \mathrm{DCM}$ for $14 \mathrm{~h}$. The crude product was purified by column chromatography on $\mathrm{SiO}_{2}$ (petroleum ether/DCM $\left.=1: 1\right)$ to afford pure $2 \mathrm{q}(2.3 \mathrm{~g}, 8.12 \mathrm{mmol}$, $75 \%)$.

Appearance: white solid.

TLC: $R_{f}=0.55$ (petroleum ether/EtOAc $=85: 15$, UV-active and stains in permanganate).

m.pt. $=105.8-110.6^{\circ} \mathrm{C}$.

${ }^{1} \mathrm{H}-\mathrm{NMR}\left(400 \mathrm{MHz}, \mathrm{CDCl}_{3}\right) \delta(\mathrm{ppm})=7.90-7.84(\mathrm{~m}, 2 \mathrm{H}), 7.81-7.75(\mathrm{~m}, 2 \mathrm{H}), 6.28-6.18(\mathrm{~m}$, $2 \mathrm{H}), 3.49-3.46(\mathrm{~m}, 1 \mathrm{H}), 3.31(\mathrm{dt}, J=9.2,3.8 \mathrm{~Hz}, 1 \mathrm{H}), 3.05-2.98(\mathrm{~m}, 1 \mathrm{H}), 2.13-2.01(\mathrm{~m}, 1 \mathrm{H})$, $1.60-1.52(\mathrm{~m}, 2 \mathrm{H}), 1.41-1.34(\mathrm{~m}, 1 \mathrm{H})$.

${ }^{13} \mathrm{C}-\mathrm{NMR}\left(101 \mathrm{MHz}, \mathrm{CDCl}_{3}\right) \delta(\mathrm{ppm})=170.9,162.1,138.2,134.7,134.6,132.2,129.0,123.9$, $123.9,49.7,46.5,42.6,40.7,29.6$.

HRMS: could not be obtained due to poor ionization.

Synthesis of 1,3-dioxoisoindolin-2-yl 2-(1-benzyl-1H-indol-3-yl)acetate (2r)
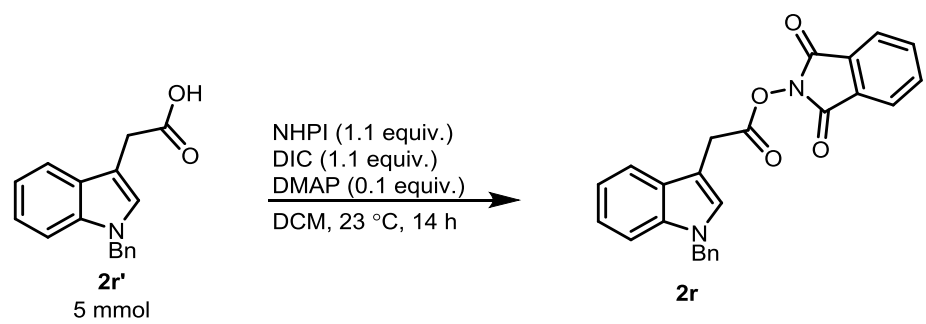

General procedure A1 was applied using 2-(1-benzyl-1H-indol-3-yl)acetic acid (2r'; $1.325 \mathrm{~g}$, $5.0 \mathrm{mmol}, 1.0$ equiv.), $N$-hydroxyphthalimide (S1; $0.896 \mathrm{~g}, 5.5 \mathrm{mmol}, 1.1$ equiv.), $N, N^{\prime}-$ diisopropylcarbodiimide ( $0.694 \mathrm{~g}, 5.5 \mathrm{mmol}, 1.1$ equiv.) and DMAP (61 mg, $0.5 \mathrm{mmol}, 0.1$ equiv.) in $30 \mathrm{~mL} \mathrm{DCM}$ for $14 \mathrm{~h}$. The crude product was purified by column chromatography on $\mathrm{SiO}_{2}$ (petroleum ether/DCM = 1:1) to afford pure $2 \mathrm{r}(1.8 \mathrm{~g}, 4.38 \mathrm{mmol}, 88 \%$ ).

Appearance: white solid.

TLC: $R_{f}=0.4$ (petroleum ether/EtOAc $=7: 3, \mathrm{UV}$-active and stains violet in vanillin). m.pt. $=142.8-146.1^{\circ} \mathrm{C}$

${ }^{1} \mathrm{H}-\mathrm{NMR}\left(400 \mathrm{MHz}, \mathrm{CDCl}_{3}\right) \delta(\mathrm{ppm})=7.89-7.87(\mathrm{~m}, 2 \mathrm{H}), 7.79-7.77(\mathrm{~m}, 2 \mathrm{H}), 7.69(\mathrm{dd}, J=7.1$, $2.0 \mathrm{~Hz}, 1 \mathrm{H}), 7.33-7.22(\mathrm{~m}, 5 \mathrm{H}), 7.21-7.13(\mathrm{~m}, 4 \mathrm{H}), 5.32(\mathrm{~s}, 2 \mathrm{H}), 4.16(\mathrm{~s}, 2 \mathrm{H})$.

${ }^{13} \mathrm{C}-\mathrm{NMR}\left(101 \mathrm{MHz}, \mathrm{CDCl}_{3}\right) \delta(\mathrm{ppm})=167.9,161.9,137.3,136.5,134.7,129.0,128.8,127.7$, $127.5,126.9,124.0,122.3,119.8,118.9,109.9,105.0,50.2,28.1$. 
HRMS (ESI-TOF) calc'd for $\mathrm{C}_{25} \mathrm{H}_{18} \mathrm{~N}_{2} \mathrm{O}_{4} \mathrm{Na}[\mathrm{M}+\mathrm{Na}]^{+}: 433.1159$, found: 433.1155 .

Synthesis of NHPI-ester of D-biotin (2s)
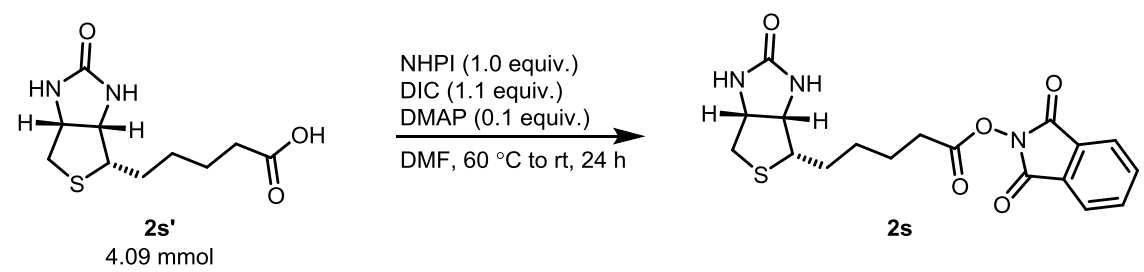

D-biotin (2s; $1.0 \mathrm{~g}, 4.09 \mathrm{mmol}, 1.0$ equiv.), $N$-hydroxyphthalimide (S1; $0.67 \mathrm{~g}, 4.09 \mathrm{mmol}, 1.0$ equiv.) and DMAP (50 mg, $0.04 \mathrm{mmol}, 0.1$ equiv.) were dissolved in hot DMF $\left(60^{\circ} \mathrm{C}\right)(0.13 \mathrm{M})$ in a round bottomed flask. $N, N^{\prime}$-diisopropylcarbodiimide $(0.93 \mathrm{~g}, 4.502 \mathrm{mmol}, 1.1$ equiv.) was added and the solution was stirred for $24 \mathrm{~h}$ during which time a white precipitate was formed. The reaction mixture was filtered, the solvent was evaporated $\left(70{ }^{\circ} \mathrm{C}\right.$ water bath, $\left.13 \mathrm{mbar}\right)$, and the residue was triturated with diethyl ether. The white precipitate was filtered and washed with diethyl ether to give $2 \mathrm{~s}$ ( $1.35 \mathrm{~g}, 3.46 \mathrm{mmol}, 85 \%$ ).

Appearance: white powder.

TLC: $R_{f}=0.55$ (DCM/ methanol = 9:1, UV-active and stains in permanganate).

m.pt. $=180.2-185.5^{\circ} \mathrm{C}$.

${ }^{1} \mathrm{H}-\mathrm{NMR}\left(400 \mathrm{MHz}, \mathrm{CDCl}_{3}\right) \delta(\mathrm{ppm})=7.89-7.86(\mathrm{~m}, 2 \mathrm{H}), 7.81-7.77(\mathrm{~m}, 2 \mathrm{H}), 6.08(\mathrm{~s}, 1 \mathrm{H}), 5.26$ $(\mathrm{s}, 1 \mathrm{H}), 4.55-4.51(\mathrm{~m}, 1 \mathrm{H}), 4.37-4.33(\mathrm{~m}, 1 \mathrm{H}), 3.20(\mathrm{ddd}, J=8.2,6.7,4.6 \mathrm{~Hz}, 1 \mathrm{H}), 2.95-2.88$ $(\mathrm{m}, 2 \mathrm{H}), 2.77-2.70(\mathrm{~m}, 2 \mathrm{H}), 1.86(\mathrm{p}, J=7.4 \mathrm{~Hz}, 2 \mathrm{H}), 1.80-1.71(\mathrm{~m}, 2 \mathrm{H}), 1.61-1.53(\mathrm{~m}, 2 \mathrm{H})$.

${ }^{13} \mathrm{C}-\mathrm{NMR}\left(101 \mathrm{MHz}, \mathrm{CDCl}_{3}\right) \delta(\mathrm{ppm})=169.8,163.8,162.2,135.0,129.0,123.7,62.0,60.3,55.6$, 40.7, 30.8, 28.3, 28.1, 24.7.

HRMS: could not be obtained due to poor ionization.

Synthesis of 1,3-dioxoisoindolin-2-yl (1R,4R)-7,7-dimethyl-2-oxobicyclo[2.2.1]heptane-1carboxylate $(2 \mathrm{x})$
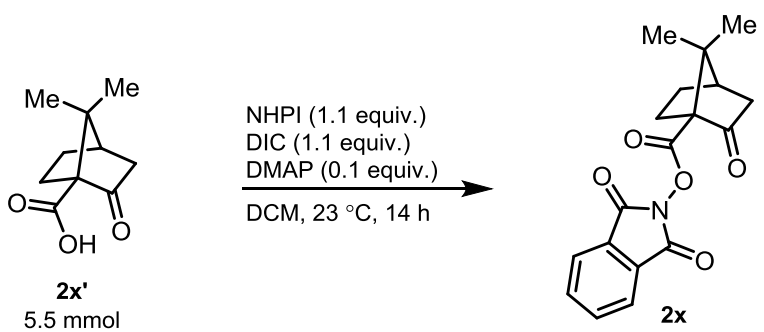

General procedure A1 was applied using (1R,4R)-7,7-dimethyl-2-oxobicyclo[2.2.1] heptane-1carboxylic acid (2x'; $1.0 \mathrm{~g}, 5.5 \mathrm{mmol}, 1.0$ equiv.), $N$-hydroxyphthalimide (S1; $1.0 \mathrm{~g}, 6.0 \mathrm{mmol}$, 1.1 equiv.), $N, N^{\prime}$-diisopropylcarbodiimide ( $0.8 \mathrm{~g}, 6.0 \mathrm{mmol}, 1.1$ equiv.) and DMAP (67 mg, 0.55 mmol, 0.1 equiv.) in $50 \mathrm{~mL}$ DCM for $14 \mathrm{~h}$. The crude product was purified by column chromatography on $\mathrm{SiO}_{2}$ (petroleum ether/DCM = 1:1) to afford pure $\mathbf{2 x}(1.6 \mathrm{~g}, 4.89 \mathrm{mmol}$, $89 \%)$.

Appearance: white solid. 
TLC: $R_{f}=0.55$ (petroleum ether/EtOAc $=6: 4$, UV-active).

m.pt. $=128.2-133.8^{\circ} \mathrm{C}$.

${ }^{1} \mathrm{H}-\mathrm{NMR}\left(400 \mathrm{MHz}, \mathrm{CDCl}_{3}\right) \delta(\mathrm{ppm})=7.90-7.87(\mathrm{~m}, 2 \mathrm{H}), 7.81-7.77(\mathrm{~m}, 2 \mathrm{H}), 2.69-2.56(\mathrm{~m}$, $2 \mathrm{H}), 2.23(\mathrm{t}, J=4.4 \mathrm{~Hz}, 1 \mathrm{H}), 2.20-2.11(\mathrm{~m}, 1 \mathrm{H}), 2.09(\mathrm{~d}, J=18.4 \mathrm{~Hz}, 1 \mathrm{H}), 2.03-1.97(\mathrm{~m}, 1 \mathrm{H})$, $1.55(\mathrm{td}, J=9.1,4.7 \mathrm{~Hz}, 1 \mathrm{H}), 1.27(\mathrm{~d}, J=9.5 \mathrm{~Hz}, 6 \mathrm{H})$.

${ }^{13} \mathrm{C}-\mathrm{NMR}\left(101 \mathrm{MHz}, \mathrm{CDCl}_{3}\right) \delta(\mathrm{ppm})=207.7,166.2,161.7,134.7,129.0,123.9,66.8,51.0,44.5$, 43.9, 26.7, 26.3, 20.9, 19.6.

HRMS (ESI-TOF) calc'd for $\mathrm{C}_{18} \mathrm{H}_{17} \mathrm{NO}_{5} \mathrm{Na}[\mathrm{M}+\mathrm{Na}]^{+}: 350.0999$, found: 350.0995 .

Synthesis of NHPI ester of derivative of 186-Glycyrrhetinic acid (2aa)
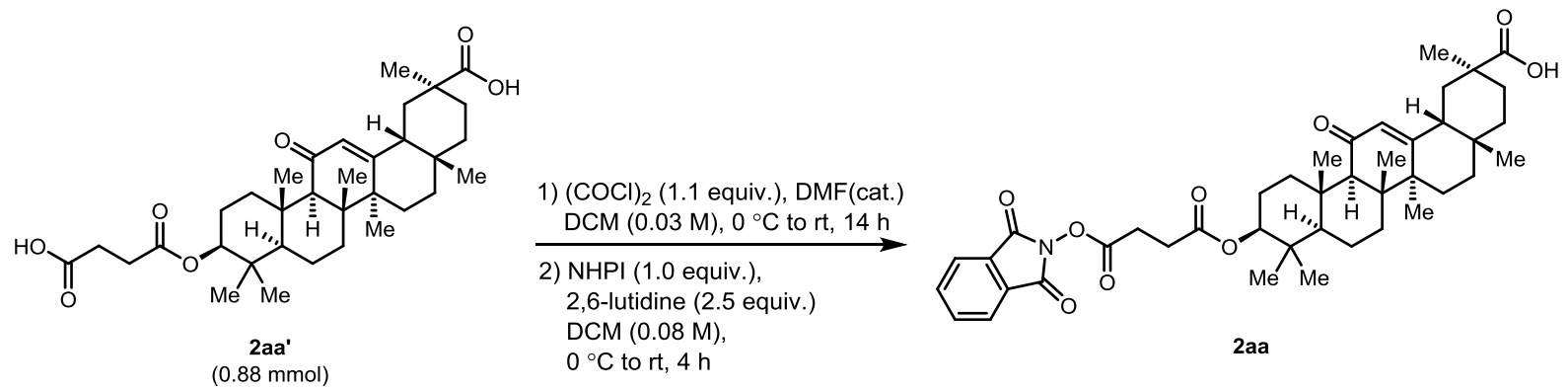

To a suspension of $(3 \beta, 18 \beta, 20 \beta)$-3-(3-Carboxy-1-oxopropoxy)-11-oxo-olean-12-en-29-oic acid (2aa', $500 \mathrm{mg}, 0.88 \mathrm{mmol}, 1.0$ equiv.) in dry DCM ( $25 \mathrm{~mL}$ ), DMF ( $3 \mu \mathrm{L}, 0.04 \mathrm{mmol}, 0.04$ equiv.) was added under argon. The suspension was placed in an ice-water bath and stirred for 10 min. Then, oxalyl chloride ( $83 \mu \mathrm{L}, 0.96 \mathrm{mmol}, 1.1$ equiv.) was added dropwise over $30 \mathrm{~min}$ (Note: gas evolution was observed and this step should be performed in a well-ventilated fume hood). After the addition, the mixture was stirred at $0{ }^{\circ} \mathrm{C}$ for $20 \mathrm{~min}$ before the ice-bath was removed. The mixture was stirred at room temperature overnight. Volatiles were removed under reduced pressure and fresh dry DCM $(15 \mathrm{~mL})$ was added. The resulting solution was placed in an ice-bath under argon. Then, a briefly sonicated solution of $\mathrm{N}$ hydroxyphthalimide S1 (143 mg, $0.88 \mathrm{mmol}, 1.0$ equiv.) and 2,6-lutidine ( $254 \mu \mathrm{L}, 2.2 \mathrm{mmol}$, 2.5 equiv.) in dry DCM (10 mL) was added slowly. The reaction was allowed to reach room temperature over $30 \mathrm{~min}$ and further stirred for $4 \mathrm{~h}$ at this temperature. Then the volume of DCM was reduced to approx. $20 \%$ under reduced pressure and the crude yellow oil was purified by column chromatography on $\mathrm{SiO}_{2}(\mathrm{DCM} / \mathrm{EtOAc}=10: 1$ to $5: 1)$ to obtain the title compound 2 aa (200 mg, $0.28 \mathrm{mmol}, 32 \%)$.

Appearance: white solid.

TLC: $R_{f}=0.45(D C M / M e O H=10: 1$, UV-active).

m.pt. $=248.9-251.3^{\circ} \mathrm{C}$.

${ }^{1} \mathrm{H}-\mathrm{NMR}\left(400 \mathrm{MHz}, \mathrm{CDCl}_{3}\right) \delta(\mathrm{ppm})=7.90-7.85(\mathrm{~m}, 2 \mathrm{H}), 7.81-7.75(\mathrm{~m}, 2 \mathrm{H}), 5.71(\mathrm{~s}, 1 \mathrm{H}), 4.58$ $(\mathrm{dd}, J=11.8,4.7 \mathrm{~Hz}, 1 \mathrm{H}), 3.09-2.96(\mathrm{~m}, 2 \mathrm{H}), 2.84-2.71(\mathrm{~m}, 3 \mathrm{H}), 2.36(\mathrm{~s}, 1 \mathrm{H}), 2.25-2.11(\mathrm{~m}$, $1 \mathrm{H}), 2.09-1.53(\mathrm{~m}, 9 \mathrm{H}), 1.48-1.29(\mathrm{~m}, 8 \mathrm{H}), 1.25-0.97(\mathrm{~m}, 13 \mathrm{H}), 0.92-0.75(\mathrm{~m}, 10 \mathrm{H})$.

${ }^{13} \mathrm{C}-\mathrm{NMR}\left(101 \mathrm{MHz}, \mathrm{CDCl}_{3}\right) \delta(\mathrm{ppm})=200.5,182.1,170.9,169.7,168.8,161.8,134.9,129.0$, 128.5, 124.1, 81.7, 61.8, 55.1, 48.4, 45.6, 43.9, 43.3, 40.9, 38.9, 38.2, 37.8, 37.0, 32.8, 32.0, $31.0,29.3,28.7,28.6,28.2,26.6,26.5,23.6,23.5,18.8,17.5,16.9,16.5$. 
HRMS (ESI-TOF) calc'd for $\mathrm{C}_{42} \mathrm{H}_{53} \mathrm{NO}{ }_{9} \mathrm{Na}[\mathrm{M}+\mathrm{Na}]^{+}:$738.3613, found: 738.3617 .

\section{Optimization studies and control experiments}

Table S1: Initial exploration of the reaction parameters

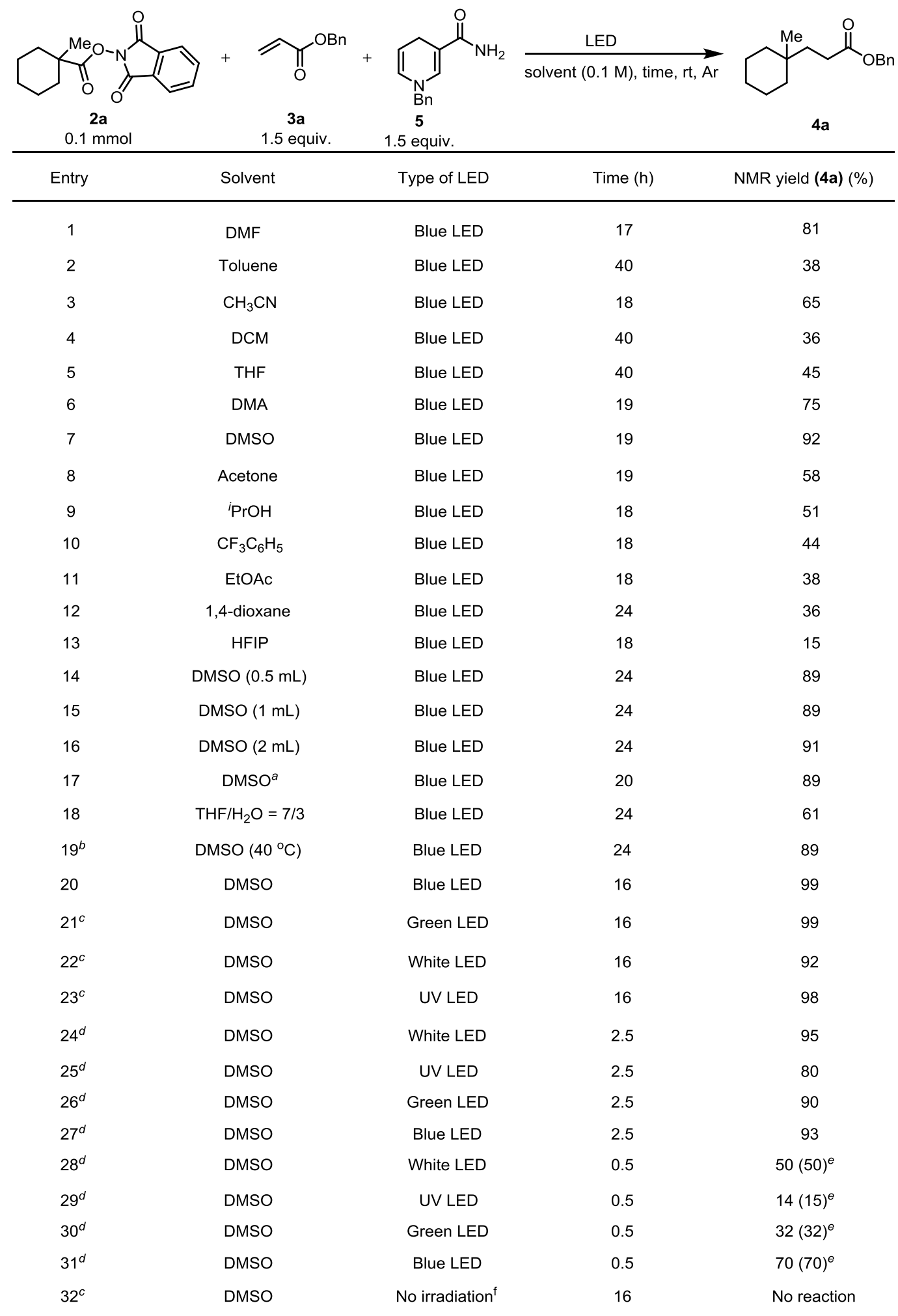

a) not degassed via sonication; b) $\left[\mathrm{Ru}(\mathrm{bpy})_{3}\right] \mathrm{Cl}_{2}(2 \mathrm{~mol} \%)$; c) I $=0.3 \mathrm{~A}$; d) I=0.1 A;

e) conversion $\mathrm{f}$ ) reaction was covered in aluminium foil 
Table S2: Study of control experiments

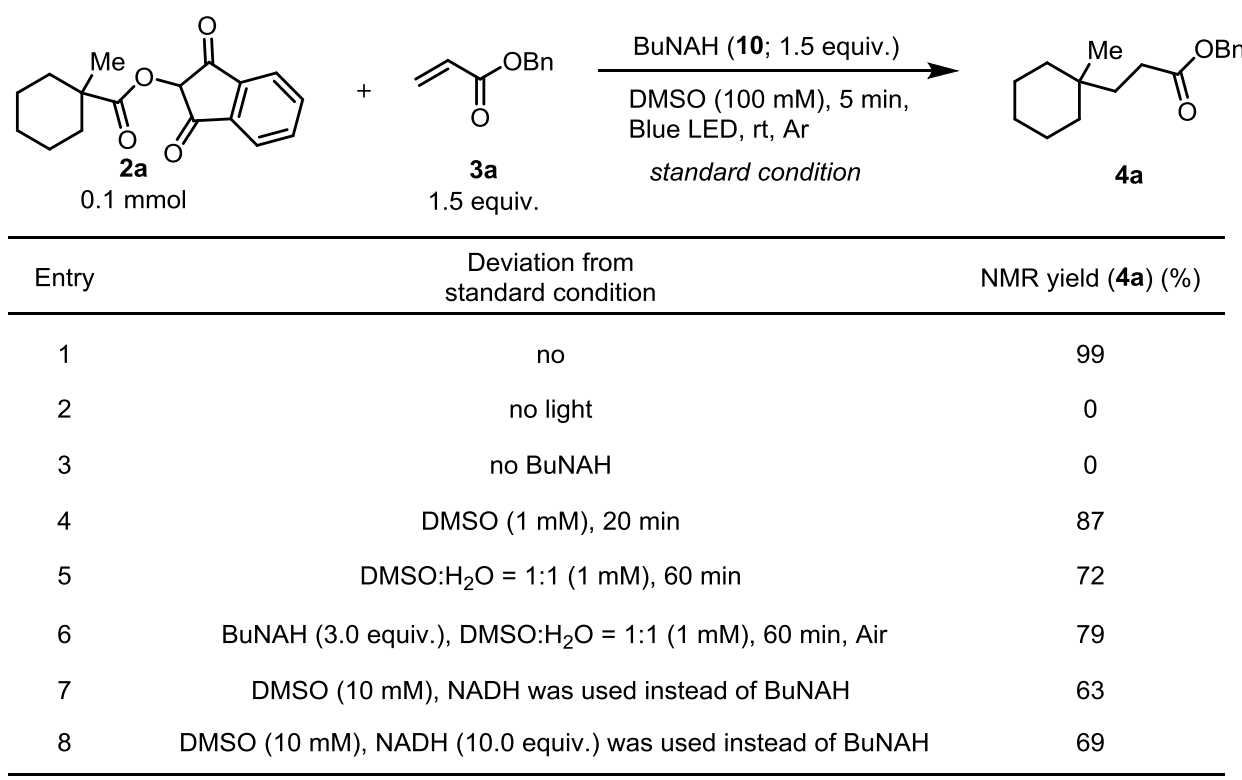

Table S3: Stability of the BuNAH (10) as a solution in DMSO

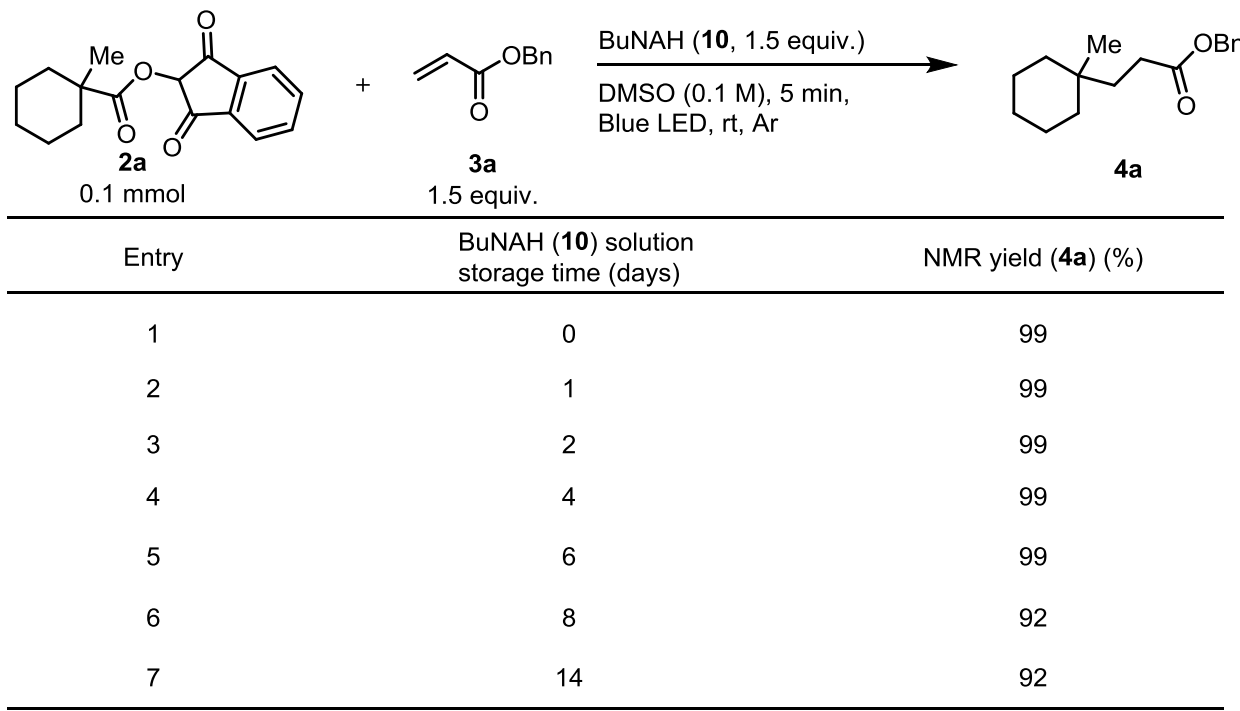

BuNAH 10 was dissolved in DMSO $(0.1 \mathrm{M})$ and stored in the freezer at $-20^{\circ} \mathrm{C}$

\section{Scope studies}

General Procedure (B1): Decarboxylative coupling of redox-active carboxylates with electron-poor olefins using BuNAH (10) and blue light

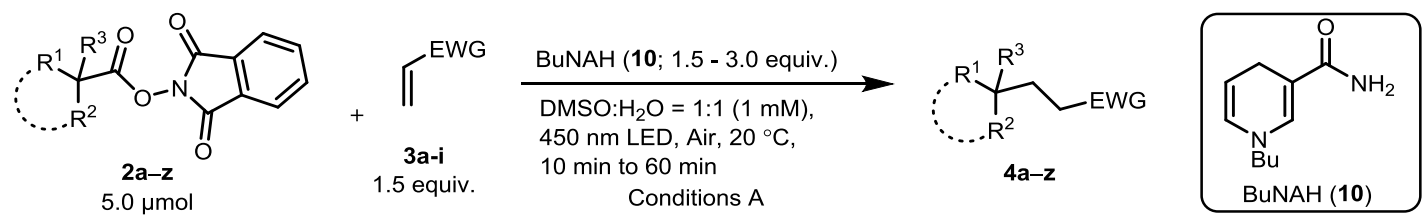

The stock solution of the redox-active ester $\mathbf{2 a - z}(5.0 \mu \mathrm{mol}, 1.0$ equiv.), Michael acceptor $3 \mathrm{a}-\mathbf{i}$ (7.5 $\mu \mathrm{mol}, 1.5$ equiv.), and 1-butyl-1,4-dihydropyridine-3-carboxamide (10; $16.2 \mathrm{mg}, 15.0$ 
$\mu$ mol, 3.0 equiv.) were prepared in DMSO $(1.2 \mathrm{~mL})$ in three different $2 \mathrm{~mL}$ vials. Next, an aliquot $(0.2 \mathrm{~mL}$, unless otherwise specified) of each stock solution was injected in a $7 \mathrm{~mL}$ vial equipped with a stirring bar. DMSO $(1.9 \mathrm{~mL})$ was added to the mixture, followed by the addition of ice-cold water $(2.5 \mathrm{~mL})$. The vial was closed with a sure seal septum, the mixture was stirred, and illuminated under blue light for the specified time at $20^{\circ} \mathrm{C}$. After completion, the reaction mixture was diluted with water $(10 \mathrm{~mL})$ and EtOAc $(15 \mathrm{~mL})$ was added. The organic layer was separated and the aqueous layer was extracted with EtOAc ( $2 \times 15 \mathrm{~mL})$. The combined organic layer was washed with brine $(10 \mathrm{~mL})$, dried over $\mathrm{Na}_{2} \mathrm{SO}_{4}$, and concentrated under reduced pressure to afford the crude 4a-z. ${ }^{1} \mathrm{H}-\mathrm{NMR}$ was measured in the presence of 1,1,2,2-tetrachloroethane as an internal standard to determine the yield of the crude product.

General Procedure (B2): Decarboxylative coupling of redox-active carboxylates with electron-poor olefins using BuNAH (10) and blue light NADH (11) and blue
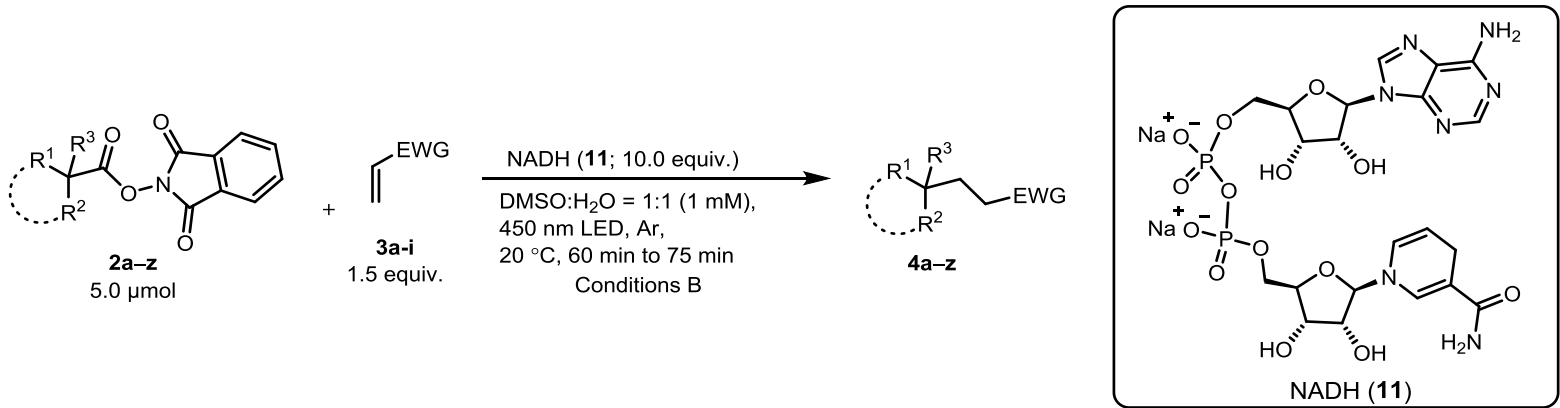

A $7 \mathrm{~mL}$ vial equipped with a stirring bar was evacuated and refilled with argon (three cycles).

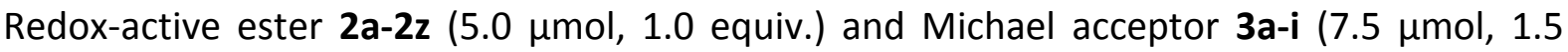
equiv.) were added in form of a stock solution (0.2 mL in DMSO). NADH (11; $35.0 \mathrm{mg}, 50.0$ $\mu \mathrm{mol}, 10.0$ equiv.) dissolved in DMSO $(2.1 \mathrm{~mL})$ was added to the mixture, followed by the addition of ice-cold water $(2.5 \mathrm{~mL})$. The resulting mixture was stirred and illuminated under blue light for the specified time at $20^{\circ} \mathrm{C}$. The reaction mixture was diluted with water $(10 \mathrm{~mL})$ and EtOAc $(15 \mathrm{~mL})$ was added. The organic layer was separated and the aqueous layer was extracted with EtOAc $(2 \times 15 \mathrm{~mL})$. The combined organic layer was washed with brine $(10 \mathrm{~mL})$, dried over $\mathrm{Na}_{2} \mathrm{SO}_{4}$, and concentrated under reduced pressure to afford the crude $4 \mathbf{a}-\mathbf{z} .{ }^{1} \mathrm{H}-$ NMR was measured in the presence of 1,1,2,2-tetrachloroethane as an internal standard to determine the yield of the crude product.

General procedure (B3): Preparative decarboxylative coupling of redox-active carboxylates with electron-poor olefins BuNAH (10) and blue light.
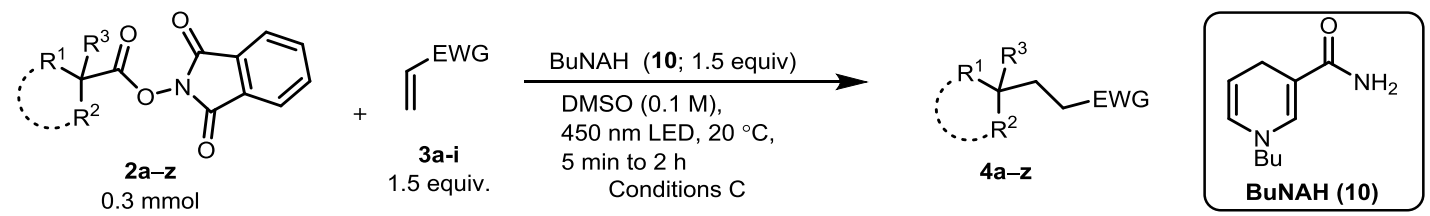

Redox-active ester $\mathbf{2 a - 2 z}$ (0.30 mmol, 1.0 equiv.) and 1-butyl-1,4-dihydropyridine-3carboxamide (10; $81.0 \mathrm{mg}, 0.45 \mathrm{mmol}, 1.5$ equiv.) were weighed in a $7 \mathrm{~mL}$ oven-dried vial equipped with a magnetic stirring bar. The vial was closed with a sure seal septum, followed by evacuation and refilling with argon (three times). Then anhydrous DMSO ( $3 \mathrm{~mL}$ ) was added 
followed by addition of Michael acceptor 3a-i ( $0.45 \mathrm{mmol}, 1.5$ equiv.). The mixture was then irradiated with blue LEDs for $5 \mathrm{~min}$ to $2 \mathrm{~h}$ at $20^{\circ} \mathrm{C}$. The reaction mixture was diluted with DCM $(15 \mathrm{~mL})$, then water $(15 \mathrm{~mL})$ and brine $(10 \mathrm{~mL})$ were added to it. The organic layer was separated and the aqueous layer was extracted with DCM $(2 \times 15 \mathrm{~mL})$. The combined organic layer was dried over $\mathrm{Na}_{2} \mathrm{SO}_{4}$ and concentrated under reduced pressure. The crude was purified by column chromatography on $\mathrm{SiO}_{2}$ to afford $4 \mathrm{a}-4 \mathrm{aa}$.

Synthesis of benzyl 3-(1-methylcyclohexyl)propanoate (4a)

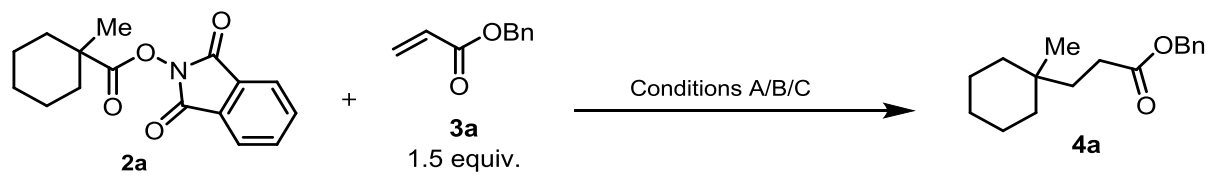

General procedure B1 was applied using 1,3-dioxoisoindolin-2-yl 1-methylcyclohexane-1carboxylate ( $2 \mathrm{a} ; 1.43 \mathrm{mg}, 5.0 \mu \mathrm{mol}, 1.0$ equiv.), benzyl acrylate (3a; $1.21 \mathrm{mg}, 7.5 \mu \mathrm{mol}, 1.5$ equiv.), 1-butyl-1,4-dihydropyridine-3-carboxamide (10; $2.71 \mathrm{mg}, 0.015 \mathrm{mmol}, 3.0$ equiv.) in a mixture of DMSO $(2.5 \mathrm{~mL})$ and water $(2.5 \mathrm{~mL})$ for 60 min under air. The crude yield was measured by ${ }^{1} \mathrm{H}-\mathrm{NMR}$ in presence of 1,1,2,2-tetrachloroethane to obtain $4 \mathrm{a}$ in $79 \%$.

General procedure B2 was applied using 1,3-dioxoisoindolin-2-yl 1-methylcyclohexane-1carboxylate (2a; $1.43 \mathrm{mg}, 5.0 \mu \mathrm{mol}, 1.0$ equiv.), benzyl acrylate (3a; $1.21 \mathrm{mg}, 7.5 \mu \mathrm{mol}, 1.5$ equiv.), NADH (11; $35.0 \mathrm{mg}, 0.05 \mathrm{mmol}, 10.0$ equiv.) in a mixture of DMSO (2.5 mL) and water $(2.5 \mathrm{~mL})$ for $60 \mathrm{~min}$ under argon. The crude yield was measured by ${ }^{1} \mathrm{H}-\mathrm{NMR}$ in presence of 1,1,2,2-tetrachloroethane to obtain 4 a in $69 \%$.

Preparative procedure B3 was applied using 1,3-dioxoisoindolin-2-yl 1-methylcyclohexane-1carboxylate (2a; $86 \mathrm{mg}, 0.30 \mathrm{mmol}, 1.0$ equiv.), benzyl acrylate (3a; $73 \mathrm{mg}, 0.45 \mathrm{mmol}, 1.5$ equiv.), 1-butyl-1,4-dihydropyridine-3-carboxamide (10; $81 \mathrm{mg}, 0.45 \mathrm{mmol}, 1.5$ equiv.) in 3 $\mathrm{mL}$ DMSO for $5 \mathrm{~min}$. The crude product was purified by column chromatography on $\mathrm{SiO}_{2}$ (petroleum ether/DCM = 5:1 to $2: 1$ ) to afford $4 a(68.0 \mathrm{mg}, 0.26 \mathrm{mmol}, 87 \%)$.

Appearance: colorless oil.

TLC: $R_{f}=0.22$ (pentane/DCM = 2:1, UV-active and stains in PMA).

${ }^{1} \mathrm{H}-\mathrm{NMR}\left(400 \mathrm{MHz}, \mathrm{CDCl}_{3}\right) \delta(\mathrm{ppm})=7.40-7.30(\mathrm{~m}, 5 \mathrm{H}), 5.12(\mathrm{~s}, 2 \mathrm{H}), 2.35-2.27(\mathrm{~m}, 2 \mathrm{H}), 1.64$ $-1.58(\mathrm{~m}, 2 \mathrm{H}), 1.48-1.37(\mathrm{~m}, 5 \mathrm{H}), 1.36-1.28(\mathrm{~m}, 1 \mathrm{H}), 1.26-1.21(\mathrm{~m}, 4 \mathrm{H}), 0.85(\mathrm{~s}, 3 \mathrm{H})$.

${ }^{13} \mathrm{C}-\mathrm{NMR}\left(101 \mathrm{MHz}, \mathrm{CDCl}_{3}\right) \delta(\mathrm{ppm})=174.4,136.1,128.5,128.2,128.2,66.2,37.5,36.7,32.3$, 29.0, 26.4, 24.4, 22.0.

All the data are in accordance with the literature. ${ }^{[2]}$

Synthesis of methyl 3-(1-methylcyclohexyl)propanoate (4b)<smiles>[M]C1(C(=O)ON2C(=O)c3ccccc3C2=O)CCCCC1</smiles>

$2 a$

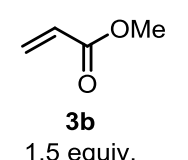

Conditions A/B/C

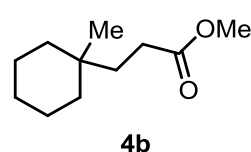

$4 b$

General procedure B1 was applied using 1,3-dioxoisoindolin-2-yl 1-methylcyclohexane-1carboxylate (2a; $1.43 \mathrm{mg}, 5.0 \mu \mathrm{mol}, 1.0$ equiv.), methyl acrylate (3b; $0.65 \mathrm{mg}, 7.5 \mu \mathrm{mol}, 1.5$ 
equiv.), 1-butyl-1,4-dihydropyridine-3-carboxamide (10; $2.71 \mathrm{mg}, 0.015 \mathrm{mmol}, 3.0$ equiv.) in a mixture of DMSO $(2.5 \mathrm{~mL})$ and water $(2.5 \mathrm{~mL})$ for 60 min under argon. The crude yield was measured by ${ }^{1} \mathrm{H}-\mathrm{NMR}$ in presence of 1,1,2,2-tetrachloroethane to obtain $\mathbf{4 b}$ in $\mathbf{7 2} \%$.

General procedure B2 was applied using 1,3-dioxoisoindolin-2-yl 1-methylcyclohexane-1carboxylat (2a; $1.43 \mathrm{mg}, 5.0 \mu \mathrm{mol}, 1.0$ equiv.), methyl acrylate (3b; $0.65 \mathrm{mg}, 7.5 \mu \mathrm{mol}, 1.5$ equiv.), NADH (11; $35.0 \mathrm{mg}, 0.05 \mathrm{mmol}, 10.0$ equiv.) in a mixture of DMSO (2.5 mL) and water $(2.5 \mathrm{~mL})$ for $60 \mathrm{~min}$ under argon. The crude yield was measured by ${ }^{1} \mathrm{H}-\mathrm{NMR}$ in presence of 1,1,2,2-tetrachloroethane to obtain $\mathbf{4 b}$ in $51 \%$.

General procedure B3 was applied using 1,3-dioxoisoindolin-2-yl 1-methylcyclohexane-1carboxylate (2a; $86 \mathrm{mg}, 0.30 \mathrm{mmol}, 1.0$ equiv.), methyl acrylate (3b; $39 \mathrm{mg}, 0.45 \mathrm{mmol}, 1.5$ equiv.), 1-butyl-1,4-dihydropyridine-3-carboxamide (10; $81 \mathrm{mg}, 0.45 \mathrm{mmol}, 1.5$ equiv.) in 3 $\mathrm{mL}$ DMSO for $5 \mathrm{~min}$. The crude product was purified by column chromatography on $\mathrm{SiO}_{2}$ (pentane/DCM = 5:1 to $2: 1$ ) to afford $4 \mathbf{b}(42.0 \mathrm{mg}, 0.23 \mathrm{mmol}, 76 \%)$.

Appearance: colorless oil.

TLC: $R_{f}=0.65$ (pentane/EtOAc $=20: 1$, stains in PMA).

${ }^{1} \mathrm{H}-\mathrm{NMR}\left(400 \mathrm{MHz}, \mathrm{CDCl}_{3}\right) \delta(\mathrm{ppm})=3.66(\mathrm{~s}, 3 \mathrm{H}), 2.31-2.21(\mathrm{~m}, 2 \mathrm{H}), 1.60-1.55(\mathrm{~m}, 2 \mathrm{H}), 1.48$ $-1.38(\mathrm{~m}, 5 \mathrm{H}), 1.35-1.27(\mathrm{~m}, 1 \mathrm{H}), 1.25-1.20(\mathrm{~m}, 4 \mathrm{H}), 0.85(\mathrm{~s}, 3 \mathrm{H})$.

${ }^{13} \mathrm{C}-\mathrm{NMR}\left(101 \mathrm{MHz}, \mathrm{CDCl}_{3}\right) \delta(\mathrm{ppm})=175.1,51.5,37.5,36.7,32.3,28.8,26.4,24.4,21.9$.

All the data are in accordance with the literature. ${ }^{[9]}$

Synthesis of N,N-dimethyl-3-(1-methylcyclohexyl)propanamide (4c)

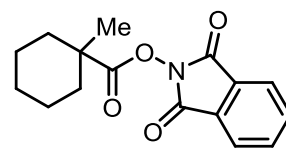

$2 a$

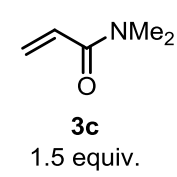

Conditions $\mathrm{A} / \mathrm{B} / \mathrm{C}$

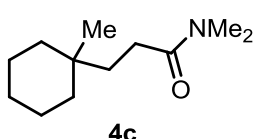

4c

General procedure B1 was applied using 1,3-dioxoisoindolin-2-yl 1-methylcyclohexane-1carboxylate (2a; $1.43 \mathrm{mg}, 5.0 \mu \mathrm{mol}, 1.0$ equiv.), $\mathrm{N}, \mathrm{N}$-dimethylacrylamide (3c; $0.75 \mathrm{mg}, 7.5$ $\mu \mathrm{mol}, 1.5$ equiv.), 1-butyl-1,4-dihydropyridine-3-carboxamide (10; $2.71 \mathrm{mg}, 0.015 \mathrm{mmol}, 3.0$ equiv.) in a mixture of DMSO $(2.5 \mathrm{~mL})$ and water $(2.5 \mathrm{~mL})$ for 60 min under argon. The crude yield was measured by ${ }^{1} \mathrm{H}-\mathrm{NMR}$ in presence of $1,1,2,2$-tetrachloroethane to obtain $4 \mathrm{c}$ in $86 \%$.

General procedure B2 was applied using 1,3-dioxoisoindolin-2-yl 1-methylcyclohexane-1carboxylate (2a; $1.43 \mathrm{mg}, 5.0 \mu \mathrm{mol}, 1.0$ equiv.), $\mathrm{N}, \mathrm{N}$-dimethylacrylamide (3c; $0.75 \mathrm{mg}, 7.5$ $\mu$ mol, 1.5 equiv.), NADH (11; $35.0 \mathrm{mg}, 0.05 \mathrm{mmol}, 10.0$ equiv.) in a mixture of DMSO (2.5 mL) and water $(2.5 \mathrm{~mL})$ for 60 min under argon. The crude yield was measured by ${ }^{1} \mathrm{H}-\mathrm{NMR}$ in presence of 1,1,2,2-tetrachloroethane to obtain $4 \mathrm{c}$ in $85 \%$.

General procedure B3 was applied using 1,3-dioxoisoindolin-2-yl 1-methylcyclohexane-1carboxylate (2a; $86 \mathrm{mg}, 0.30 \mathrm{mmol}, 1.0$ equiv.), $\mathrm{N}, \mathrm{N}$-dimethylacrylamide (3c; $45 \mathrm{mg}, 0.45$ mmol, 1.5 equiv.), 1-butyl-1,4-dihydropyridine-3-carboxamide (10; $81 \mathrm{mg}, 0.45 \mathrm{mmol}, 1.5$ equiv.) in $3 \mathrm{~mL}$ DMSO for $10 \mathrm{~min}$. The crude product was purified by column chromatography on $\mathrm{SiO}_{2}$ (petroleum ether/EtOAc = 1:1 to 1:2) to afford 4c (44 mg, $\left.0.22 \mathrm{mmol}, 74 \%\right)$.

Appearance: colorless oil. 
TLC: $R_{f}=0.50$ (pentane/EtOAC $=1: 2$, stains in PMA).

${ }^{1} \mathrm{H}-\mathrm{NMR}\left(400 \mathrm{MHz}, \mathrm{CDCl}_{3}\right) \delta(\mathrm{ppm})=3.01(\mathrm{~s}, 3 \mathrm{H}), 2.93(\mathrm{~s}, 3 \mathrm{H}), 2.29-2.21(\mathrm{~m}, 2 \mathrm{H}), 1.59-1.53$ $(\mathrm{m}, 2 \mathrm{H}), 1.48-1.37(\mathrm{~m}, 5 \mathrm{H}), 1.34-1.28(\mathrm{~m}, 1 \mathrm{H}), 1.25(\mathrm{~m}, 4 \mathrm{H}), 0.87(\mathrm{~s}, 3 \mathrm{H})$.

${ }^{13} \mathrm{C}-\mathrm{NMR}\left(101 \mathrm{MHz}, \mathrm{CDCl}_{3}\right) \delta(\mathrm{ppm})=174.0,37.6,37.4,36.9,35.4,32.4,27.9,26.4,24.6,22.0$. All the data are in accordance with the literature. ${ }^{[13]}$

Synthesis of 3-(1-methylcyclohexyl)propanal (4d)

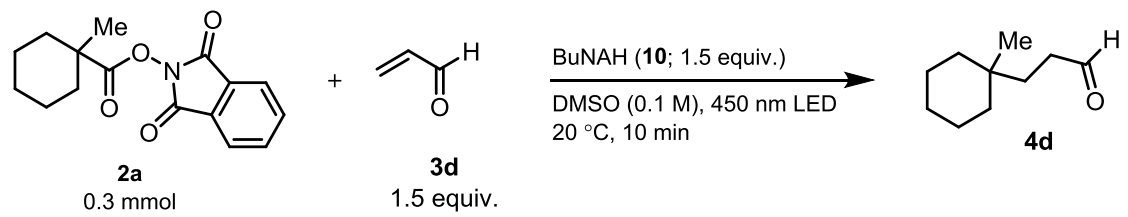

General procedure B3 was applied using 1,3-dioxoisoindolin-2-yl 1-methylcyclohexane-1carboxylate (2a; $86 \mathrm{mg}, 0.30 \mathrm{mmol}, 1.0$ equiv.), acrylaldehyde (3d; $25 \mathrm{mg}, 0.45 \mathrm{mmol}, 1.5$ equiv.), 1-butyl-1,4-dihydropyridine-3-carboxamide (10; $81 \mathrm{mg}, 0.45 \mathrm{mmol}, 1.5$ equiv.) in DMSO (3 mL) for $10 \mathrm{~min}$. The crude product was purified by column chromatography on $\mathrm{SiO}_{2}$ (petroleum ether/DCM $=2: 1$ ) to afford $4 \mathrm{~d}(19 \mathrm{mg}, 0.123 \mathrm{mmol}, 41 \%$ ).

Appearance: colorless oil.

TLC: $R_{f}=0.33$ (pentane/EtOAc $=20: 1$, stains in PMA).

${ }^{1} \mathrm{H}-\mathrm{NMR}\left(400 \mathrm{MHz}, \mathrm{CDCl}_{3}\right) \delta(\mathrm{ppm})=9.79(\mathrm{t}, J=1.9 \mathrm{~Hz}, 1 \mathrm{H}), 2.38(\mathrm{ddd}, J=10.0,6.3,2.0 \mathrm{~Hz}$, $2 \mathrm{H}), 1.57-1.53(\mathrm{~m}, 2 \mathrm{H}), 1.44(\mathrm{~m}, 5 \mathrm{H}), 1.30(\mathrm{~m}, 1 \mathrm{H}), 1.27-1.20(\mathrm{~m}, 4 \mathrm{H}), 0.85(\mathrm{~s}, 3 \mathrm{H})$.

${ }^{13} \mathrm{C}-\mathrm{NMR}\left(101 \mathrm{MHz}, \mathrm{CDCl}_{3}\right) \delta(\mathrm{ppm})=203.3,38.7,37.6,33.7,32.2,26.4,24.5,21.9$.

All the data are in accordance with the literature. ${ }^{[9]}$

Synthesis of 4-(1-methylcyclohexyl)butan-2-one (4e)

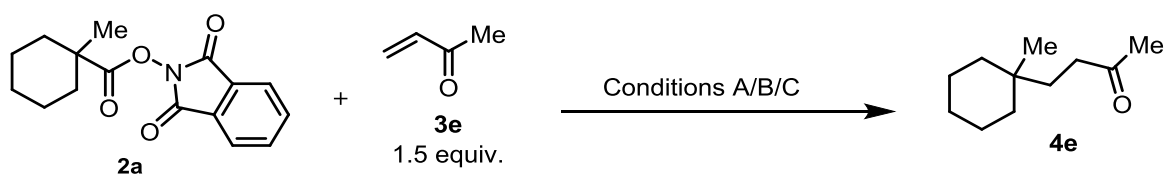

General procedure B1 was applied using 1,3-dioxoisoindolin-2-yl 1-methylcyclohexane-1carboxylate (2a; $1.43 \mathrm{mg}, 5.0 \mu \mathrm{mol}, 1.0$ equiv.), methyl vinyl ketone (3e; $0.53 \mathrm{mg}, 7.5 \mu \mathrm{mol}$, 1.5 equiv.), 1-butyl-1,4-dihydropyridine-3-carboxamide (10; $2.71 \mathrm{mg}, 0.015 \mathrm{mmol}, 3.0$ equiv.) in a mixture of DMSO $(2.5 \mathrm{~mL})$ and water $(2.5 \mathrm{~mL})$ for 60 min under air. The crude yield was measured by ${ }^{1} \mathrm{H}-\mathrm{NMR}$ in presence of 1,1,2,2-tetrachloroethane to obtain $4 \mathrm{e}$ in $68 \%$.

General procedure B2 was applied using 1,3-dioxoisoindolin-2-yl 1-methylcyclohexane-1carboxylate (2a; $1.43 \mathrm{mg}, 5.0 \mu \mathrm{mol}, 1.0$ equiv.), methyl vinyl ketone (3e; $0.53 \mathrm{mg}, 7.5 \mu \mathrm{mol}$, 1.5 equiv.), NADH (11; $35.0 \mathrm{mg}, 0.05 \mathrm{mmol}, 10.0$ equiv.) in a mixture of DMSO (2.5 mL) and water $(2.5 \mathrm{~mL})$ for 60 min under argon. The crude yield was measured by ${ }^{1} \mathrm{H}-\mathrm{NMR}$ in presence of 1,1,2,2-tetrachloroethane to obtain $4 \mathbf{e}$ in $68 \%$.

General procedure B3 was applied using 1,3-dioxoisoindolin-2-yl 1-methylcyclohexane-1carboxylate (2a; $86 \mathrm{mg}, 0.30 \mathrm{mmol}, 1.0$ equiv.), methyl vinyl ketone (3e; $32 \mathrm{mg}, 0.45 \mathrm{mmol}$, 1.5 equiv.), 1-butyl-1,4-dihydropyridine-3-carboxamide (10; $81 \mathrm{mg}, 0.45 \mathrm{mmol}, 1.5$ equiv.) in 
DMSO ( $3 \mathrm{~mL}$ ) for $5 \mathrm{~min}$. The crude product was purified by column chromatography on $\mathrm{SiO}_{2}$ (petroleum ether/DCM = 5:1 to $2: 1$ ) to afford $4 \mathrm{e}(43.9 \mathrm{mg}, 0.26 \mathrm{mmol}, 87 \%)$.

Appearance: colorless oil.

TLC: $R_{f}=0.33$ (pentane/EtOAC $=10: 1$, stains in PMA).

${ }^{1} \mathrm{H}-\mathrm{NMR}\left(400 \mathrm{MHz}, \mathrm{CDCl}_{3}\right) \delta(\mathrm{ppm})=2.39-2.33(\mathrm{~m}, 2 \mathrm{H}), 2.15(\mathrm{~s}, 3 \mathrm{H}), 1.52-1.47(\mathrm{~m}, 2 \mathrm{H}), 1.43$ $(\mathrm{m}, 5 \mathrm{H}), 1.30(\mathrm{~m}, J=8.1,4.6,2.5 \mathrm{~Hz}, 1 \mathrm{H}), 1.22(\mathrm{~m}, 4 \mathrm{H}), 0.83(\mathrm{~s}, 3 \mathrm{H})$.

${ }^{13} \mathrm{C}-\mathrm{NMR}\left(101 \mathrm{MHz} \mathrm{CDCl}_{3}\right) \delta(\mathrm{ppm})=209.9,38.3,37.6,35.5,32.2,29.9,26.4,24.6,22.0$.

All the data are in accordance with the literature. ${ }^{[2]}$

Synthesis of 3-(1-methylcyclohexyl)propanenitrile (4f)

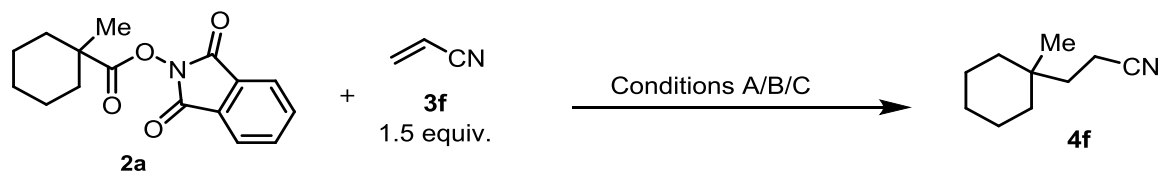

General procedure B1 was applied using 1,3-dioxoisoindolin-2-yl 1-methylcyclohexane-1carboxylate (2a; $1.43 \mathrm{mg}, 5.0 \mu \mathrm{mol}, 1.0$ equiv.), acrylonitrile (3f; $0.39 \mathrm{mg}, 7.5 \mu \mathrm{mol}, 1.5$ equiv.), 1-butyl-1,4-dihydropyridine-3-carboxamide $(10 ; 2.71 \mathrm{mg}, 0.015 \mathrm{mmol}, 3.0$ equiv.) in a mixture of DMSO $(2.5 \mathrm{~mL})$ and water $(2.5 \mathrm{~mL})$ for 60 min under air. The crude yield was measured by ${ }^{1} \mathrm{H}-\mathrm{NMR}$ in presence of 1,1,2,2-tetrachloroethane to obtain $\mathbf{4 f}$ in $66 \%$.

General procedure B2 was applied using 1,3-dioxoisoindolin-2-yl 1-methylcyclohexane-1carboxylate (2a; $1.43 \mathrm{mg}, 5.0 \mu \mathrm{mol}, 1.0$ equiv.), acrylonitrile (3f; $0.39 \mathrm{mg}, 7.5 \mu \mathrm{mol}, 1.5$ equiv.), $\operatorname{NADH}(11 ; 35.0 \mathrm{mg}, 0.05 \mathrm{mmol}, 10.0$ equiv.) in a mixture of DMSO (2.5 mL) and water (2.5 $\mathrm{mL}$ ) for 60 min under argon. The crude yield was measured by ${ }^{1} \mathrm{H}-\mathrm{NMR}$ in presence of $1,1,2,2-$ tetrachloroethane to obtain $\mathbf{4 f}$ in $68 \%$.

General procedure B3 was applied using 1,3-dioxoisoindolin-2-yl 1-methylcyclohexane-1carboxylate ( $2 \mathrm{a} ; 86 \mathrm{mg}, 0.30 \mathrm{mmol}, 1.0$ equiv.), acrylonitrile ( $3 \mathrm{f} ; 24 \mathrm{mg}, 0.45 \mathrm{mmol}, 1.5$ equiv.), 1-butyl-1,4-dihydropyridine-3-carboxamide (10; $81 \mathrm{mg}, 0.45 \mathrm{mmol}, 1.5$ equiv.) in DMSO (3 $\mathrm{mL}$ ) for $5 \mathrm{~min}$. The crude product was purified by column chromatography on $\mathrm{SiO}_{2}$ (petroleum ether/DCM = 5:1 to $2: 1$ ) to afford $\mathbf{4 f}$ (43.1 $\mathrm{mg}, 0.29 \mathrm{mmol}, 95 \%)$.

Appearance: colorless oil.

TLC: $R_{f}=0.33$ (pentane/EtOAc $=10: 1$, stains in PMA).

${ }^{1} \mathrm{H}-\mathrm{NMR}\left(400 \mathrm{MHz}, \mathrm{CDCl}_{3}\right) \delta(\mathrm{ppm})=2.30-2.22(\mathrm{~m}, 2 \mathrm{H}), 1.68-1.61(\mathrm{~m}, 2 \mathrm{H}), 1.53-1.40(\mathrm{~m}$, $5 \mathrm{H}), 1.37-1.18(\mathrm{~m}, 5 \mathrm{H}), 0.88(\mathrm{~s}, 3 \mathrm{H})$.

${ }^{13} \mathrm{C}-\mathrm{NMR}\left(101 \mathrm{MHz}, \mathrm{CDCl}_{3}\right) \delta(\mathrm{ppm})=120.7,37.4,37.1,32.6,26.2,24.1,21.8,11.7$.

All the data are in accordance with the literature. ${ }^{[2]}$

Synthesis of ((2-(1-methylcyclohexyl)ethyl)sulfonyl)benzene (4g)<smiles>CC1(C(=O)ON2C(=O)c3ccccc3C2=O)CCCCC1</smiles>

2a

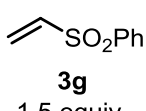
1.5 equiv.
Conditions $\mathrm{A} / \mathrm{B} / \mathrm{C}$<smiles>CC1(CCS(=O)(=O)c2ccccc2)CCCCC1</smiles> 
General procedure B1 was applied using 1,3-dioxoisoindolin-2-yl 1-methylcyclohexane-1carboxylate (2a; $1.43 \mathrm{mg}, 5.0 \mu \mathrm{mol}, 1.0$ equiv.), phenyl vinyl sulfone ( $3 \mathrm{~g} ; 1.26 \mathrm{mg}, 7.5 \mu \mathrm{mol}$, 1.5 equiv.), 1-butyl-1,4-dihydropyridine-3-carboxamide (10; $2.71 \mathrm{mg}, 0.015 \mathrm{mmol}, 3.0$ equiv.) in a mixture of DMSO $(2.5 \mathrm{~mL})$ and water $(2.5 \mathrm{~mL})$ for 60 min under air. The crude yield was measured by ${ }^{1} \mathrm{H}-\mathrm{NMR}$ in presence of 1,1,2,2-tetrachloroethane to obtain $\mathbf{4 g}$ in $70 \%$.

General procedure B2 was applied using 1,3-dioxoisoindolin-2-yl 1-methylcyclohexane-1carboxylate (2a; $1.43 \mathrm{mg}, 5.0 \mu \mathrm{mol}, 1.0$ equiv.), phenyl vinyl sulfone (3g; $1.26 \mathrm{mg}, 7.5 \mu \mathrm{mol}$, 1.5 equiv.), NADH (11; $35.0 \mathrm{mg}, 0.05 \mathrm{mmol}, 10.0$ equiv.) in a mixture of DMSO (2.5 mL) and water $(2.5 \mathrm{~mL})$ for 60 min under argon. The crude yield was measured by ${ }^{1} \mathrm{H}$-NMR in presence of 1,1,2,2-tetrachloroethane to obtain $\mathbf{4 g}$ in $96 \%$.

General procedure B3 was applied using 1,3-dioxoisoindolin-2-yl 1-methylcyclohexane-1carboxylate (2a; $86 \mathrm{mg}, 0.30 \mathrm{mmol}, 1.0$ equiv.), phenyl vinyl sulfone (3g; $75 \mathrm{mg}, 0.45 \mathrm{mmol}$, 1.5 equiv.), 1-butyl-1,4-dihydropyridine-3-carboxamide (10; $81 \mathrm{mg}, 0.45 \mathrm{mmol}, 1.5$ equiv.) in DMSO ( $3 \mathrm{~mL}$ ) for $5 \mathrm{~min}$. The crude product was purified by column chromatography on $\mathrm{SiO}_{2}$ (petroleum ether/EtOAc $=10: 1$ to $5: 1)$ to afford $4 \mathrm{~g}(66.2 \mathrm{mg}, 0.25 \mathrm{mmol}, 83 \%)$.

Appearance: colorless oil.

TLC: $R_{f}=0.51$ (pentane/EtOAC $=5: 1$, UV-active).

${ }^{1} \mathrm{H}-\mathrm{NMR}\left(400 \mathrm{MHz}, \mathrm{CDCl}_{3}\right) \delta(\mathrm{ppm})=7.94-7.86(\mathrm{~m}, 2 \mathrm{H}), 7.69-7.61(\mathrm{~m}, 1 \mathrm{H}), 7.60-7.54(\mathrm{~m}$, $2 \mathrm{H}), 3.08-2.98(\mathrm{~m}, 2 \mathrm{H}), 1.64-1.60(\mathrm{~m}, 2 \mathrm{H}), 1.41-1.33(\mathrm{~m}, 5 \mathrm{H}), 1.29-1.23(\mathrm{~m}, 1 \mathrm{H}), 1.23-$ $1.13(\mathrm{~m}, 4 \mathrm{H}), 0.80(\mathrm{~s}, 3 \mathrm{H})$.

${ }^{13} \mathrm{C}-\mathrm{NMR}\left(101 \mathrm{MHz}, \mathrm{CDCl}_{3}\right) \delta(\mathrm{ppm})=139.3,133.6,129.2,128.0,52.0,37.4,33.6,32.3,26.1$, 24.4, 21.7.

All the data are in accordance with the literature. ${ }^{[13]}$

Synthesis of 3-(2-(1-methylcyclohexyl)ethyl)pyrrolidine-2,5-dione (4h)

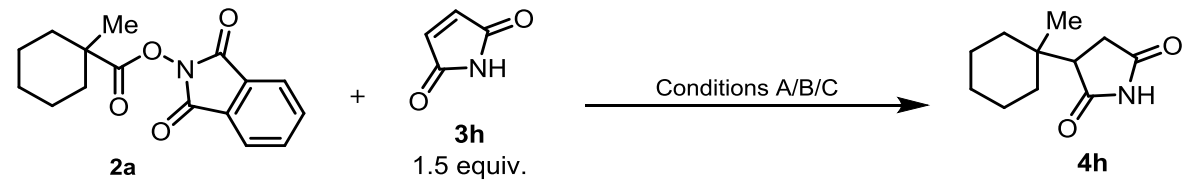

General procedure B1 was applied using 1,3-dioxoisoindolin-2-yl 1-methylcyclohexane-1carboxylate (2a; $1.43 \mathrm{mg}, 5.0 \mu \mathrm{mol}, 1.0$ equiv.), $1 \mathrm{H}$-pyrrole-2,5-dione ( $3 \mathrm{~h} ; 0.73 \mathrm{mg}, 7.5 \mu \mathrm{mol}$, 1.5 equiv.), 1-butyl-1,4-dihydropyridine-3-carboxamide (10; $2.71 \mathrm{mg}, 0.015 \mathrm{mmol}, 3.0$ equiv.) in DMSO $(20 \mathrm{mM})$ for $60 \mathrm{~min}$ under argon. The crude yield was measured by ${ }^{1} \mathrm{H}-\mathrm{NMR}$ in presence of 1,1,2,2-tetrachloroethane to obtain $\mathbf{4 h}$ in $82 \%$.

General procedure B2 was applied using 1,3-dioxoisoindolin-2-yl 1-methylcyclohexane-1carboxylate (2a; $1.43 \mathrm{mg}, 5.0 \mu \mathrm{mol}, 1.0$ equiv.), $1 \mathrm{H}$-pyrrole-2,5-dione (3h; $0.73 \mathrm{mg}, 7.5 \mu \mathrm{mol}$, 1.5 equiv.), NADH (11; $35.0 \mathrm{mg}, 0.05 \mathrm{mmol}, 10.0$ equiv.) in DMSO (20 mM) for $60 \mathrm{~min}$ under argon. The crude yield was measured by ${ }^{1} \mathrm{H}-\mathrm{NMR}$ in presence of 1,1,2,2-tetrachloroethane to obtain $\mathbf{4 h}$ in $71 \%$.

General procedure B3 was applied using 1,3-dioxoisoindolin-2-yl 1-methylcyclohexane-1carboxylate (2a; $86 \mathrm{mg}, 0.30 \mathrm{mmol}, 1.0$ equiv.), $1 \mathrm{H}$-pyrrole-2,5-dione ( $3 \mathrm{~h} ; 44 \mathrm{mg}, 0.45 \mathrm{mmol}$, 1.5 equiv.), 1-butyl-1,4-dihydropyridine-3-carboxamide (10; $81 \mathrm{mg}, 0.45 \mathrm{mmol}, 1.5$ equiv.) in 
DMSO ( $3 \mathrm{~mL}$ ) for $10 \mathrm{~min}$. The crude product was purified by column chromatography on $\mathrm{SiO}_{2}$ (petroleum ether/EtOAc $=5: 1$ to $2: 1$ ) to afford $4 \mathrm{~h}(43.1 \mathrm{mg}, 0.22 \mathrm{mmol}, 74 \%)$.

Appearance: white solid.

TLC: $R_{f}=0.42$ (Pentane/EtOAC $=2: 1$, stains in PMA).

m.pt. $=132.2-135.6^{\circ} \mathrm{C}$.

${ }^{1} \mathrm{H}-\mathrm{NMR}\left(400 \mathrm{MHz}, \mathrm{CDCl}_{3}\right) \delta(\mathrm{ppm})=8.30(\mathrm{~s}, 1 \mathrm{H}), 2.90(\mathrm{dd}, J=9.0,5.3 \mathrm{~Hz}, 1 \mathrm{H}), 2.71(\mathrm{dd}, J=$ 18.0, $9.0 \mathrm{~Hz}, 1 \mathrm{H}), 2.60(\mathrm{dd}, J=18.5,5.3 \mathrm{~Hz}, 1 \mathrm{H}), 1.69-1.40(\mathrm{~m}, 8 \mathrm{H}), 1.32(\mathrm{~m}, 2 \mathrm{H}), 0.98(\mathrm{~s}, 3 \mathrm{H})$.

${ }^{13} \mathrm{C}-\mathrm{NMR}\left(101 \mathrm{MHz}, \mathrm{CDCl}_{3}\right) \delta(\mathrm{ppm})=178.9,176.8,36.0,35.7,35.4,32.0,25.9,21.7,21.4$.

HRMS (ESI-TOF) calc'd for $\mathrm{C}_{11} \mathrm{H}_{17} \mathrm{NO}_{2}[\mathrm{M}-\mathrm{H}]::$ : 194.1187, found: 194.1185 .

Synthesis of 1-pentyl-4-(2-(phenylsulfonyl)ethyl)bicyclo[2.2.2]octane (4i)

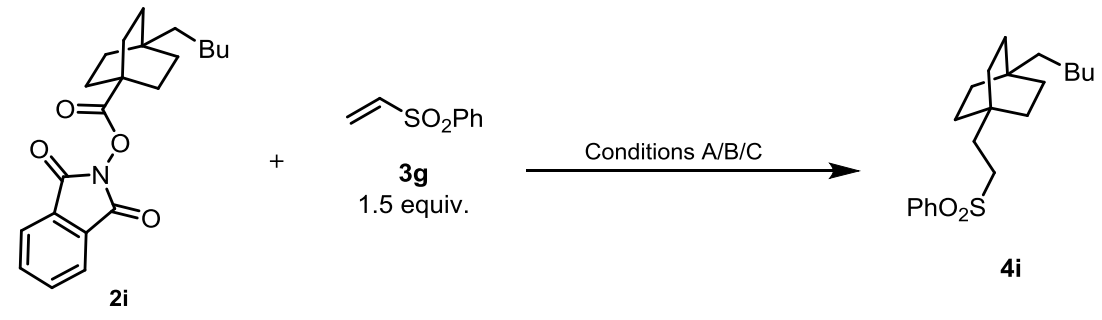

General procedure B1 was applied using 1,3-dioxoisoindolin-2-yl 4pentylbicyclo[2.2.2] octane-1-carboxylate ( $2 \mathrm{i} ; 1.84 \mathrm{mg}, 5.0 \mu \mathrm{mol}, 1.0$ equiv.), phenyl vinyl sulfone (3g; $1.26 \mathrm{mg}, 7.5 \mu \mathrm{mol}, 1.5$ equiv.), 1-butyl-1,4-dihydropyridine-3-carboxamide (10; $2.71 \mathrm{mg}, 0.015 \mathrm{mmol}, 3.0$ equiv.) in a mixture of DMSO $(2.5 \mathrm{~mL})$ and water $(2.5 \mathrm{~mL})$ for $60 \mathrm{~min}$ under air. The crude yield was measured by ${ }^{1} \mathrm{H}-\mathrm{NMR}$ in presence of 1,1,2,2-tetrachloroethane to obtain $4 \mathbf{i}$ in $47 \%$.

General procedure B2 was applied using 1,3-dioxoisoindolin-2-yl 4pentylbicyclo[2.2.2] octane-1-carboxylate ( $2 \mathrm{i} ; 1.84 \mathrm{mg}, 5.0 \mu \mathrm{mol}, 1.0$ equiv.), phenyl vinyl sulfone (3g; $1.26 \mathrm{mg}, 7.5 \mu \mathrm{mol}, 1.5$ equiv.), NADH (11; $35.0 \mathrm{mg}, 0.05 \mathrm{mmol}, 10.0$ equiv.) in a mixture of DMSO $(2.5 \mathrm{~mL})$ and water $(2.5 \mathrm{~mL})$ for $60 \mathrm{~min}$ under argon. The crude yield was measured by ${ }^{1} \mathrm{H}-\mathrm{NMR}$ in presence of 1,1,2,2-tetrachloroethane to obtain $4 \mathbf{i}$ in $58 \%$.

General procedure B3 was applied using 1,3-dioxoisoindolin-2-yl 4pentylbicyclo[2.2.2] octane-1-carboxylate (2i; $110.8 \mathrm{mg}, 0.30 \mathrm{mmol}, 1.0$ equiv.), phenyl vinyl sulfone (3g; $75 \mathrm{mg}, 0.45 \mathrm{mmol}, 1.5$ equiv.), 1-butyl-1,4-dihydropyridine-3-carboxamide (10; $81 \mathrm{mg}, 0.45 \mathrm{mmol}, 1.5$ equiv.) in $3 \mathrm{~mL}$ DMSO for $15 \mathrm{~min}$. The crude product was purified by column chromatography on $\mathrm{SiO}_{2}$ (pentane/EtOAc $=19: 1$ to 9:1) to afford $4 \mathbf{i}$ (77 $\mathrm{mg}, 0.22$ $\mathrm{mmol}, 73 \%)$.

Appearance: white solid.

TLC: $R_{f}=0.65$ (pentane/EtOAc $=5: 1$, UV-active).

m.p.: $85.7-87.7^{\circ} \mathrm{C}$.

${ }^{1} \mathrm{H}-\mathrm{NMR}\left(400 \mathrm{MHz}, \mathrm{CDCl}_{3}\right) \delta(\mathrm{ppm})=7.91-7.86(\mathrm{~m}, 2 \mathrm{H}), 7.67-7.62(\mathrm{~m}, 1 \mathrm{H}), 7.59-7.53(\mathrm{~m}$, $2 \mathrm{H}), 3.03-2.95(\mathrm{~m}, 2 \mathrm{H}), 1.52-1.45(\mathrm{~m}, 2 \mathrm{H}), 1.33-1.23(\mathrm{~m}, 14 \mathrm{H}), 1.22-1.07(\mathrm{~m}, 5 \mathrm{H}), 1.06-$ $0.97(\mathrm{~m}, 2 \mathrm{H}), 0.85(\mathrm{t}, J=7.2 \mathrm{~Hz}, 3 \mathrm{H})$. 
${ }^{13} \mathrm{C}-\mathrm{NMR}(101 \mathrm{MHz}, \mathrm{CDCl}) \delta(\mathrm{ppm})=139.3,133.5,129.2,128.0,52.2,41.5,33.3,32.8,31.0$, 30.9, 30.4, 30.4, 23.3, 22.7, 14.1.

HRMS (ESI-TOF) calc'd for $\mathrm{C}_{21} \mathrm{H}_{32} \mathrm{O}_{2} \mathrm{SNa}[\mathrm{M}+\mathrm{Na}]^{+}: 371.2015$, found: 371.2010.

Synthesis of benzyl 3-adamantan-1-yl propanoate (4j)

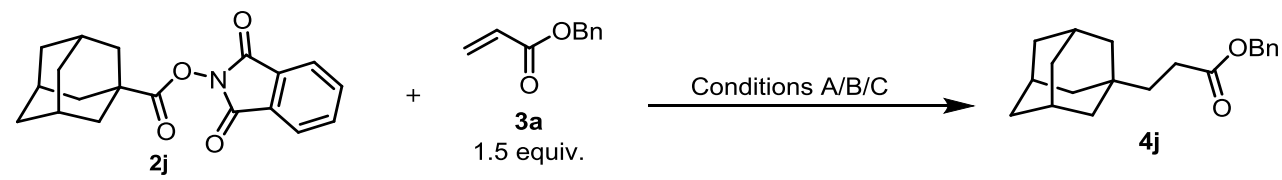

General procedure B1 was applied using 1,3-dioxoisoindolin-2-yl $(3 r, 5 r, 7 r)$-adamantane-1carboxylate (2j; $1.62 \mathrm{mg}, 5.0 \mu \mathrm{mol}, 1.0$ equiv.), benzyl acrylate (3a; $1.21 \mathrm{mg}, 7.5 \mu \mathrm{mol}, 1.5$ equiv.), 1-butyl-1,4-dihydropyridine-3-carboxamide (10; $2.71 \mathrm{mg}, 0.015 \mathrm{mmol}, 3.0$ equiv.) in a mixture of DMSO $(2.5 \mathrm{~mL})$ and water $(2.5 \mathrm{~mL})$ for 60 min under air. The crude yield was measured by ${ }^{1} \mathrm{H}-\mathrm{NMR}$ in presence of 1,1,2,2-tetrachloroethane to obtain $\mathbf{4} \mathbf{j}$ in $50 \%$.

General procedure B2 was applied using 1,3-dioxoisoindolin-2-yl (3r,5r,7r)-adamantane-1carboxylate (2j; $1.62 \mathrm{mg}, 5.0 \mu \mathrm{mol}, 1.0$ equiv.), benzyl acrylate (3a; $1.21 \mathrm{mg}, 7.5 \mu \mathrm{mol}, 1.5$ equiv.), NADH (11; $35.0 \mathrm{mg}, 0.05 \mathrm{mmol}, 10.0$ equiv.) in a mixture of DMSO (2.5 mL) and water $(2.5 \mathrm{~mL})$ for $60 \mathrm{~min}$ under argon. The crude yield was measured by ${ }^{1} \mathrm{H}-\mathrm{NMR}$ in presence of 1,1,2,2-tetrachloroethane to obtain $\mathbf{4 j}$ in $46 \%$.

General procedure B3 was applied using 1,3-dioxoisoindolin-2-yl $(3 r, 5 r, 7 r)$-adamantane-1carboxylate (2j; $97.6 \mathrm{mg}, 0.30 \mathrm{mmol}, 1.0$ equiv.), benzyl acrylate (3a; $73 \mathrm{mg}, 0.45 \mathrm{mmol}, 1.5$ equiv.), 1-butyl-1,4-dihydropyridine-3-carboxamide (10; $81 \mathrm{mg}, 0.45 \mathrm{mmol}, 1.5$ equiv.) in DMSO ( $3 \mathrm{~mL}$ ) for $5 \mathrm{~min}$. The crude product was purified by column chromatography on $\mathrm{SiO}_{2}$ (pentane/EtOAc $=99: 1$ ) to afford $4 \mathbf{j}(71 \mathrm{mg}, 0.23 \mathrm{mmol}, 79 \%)$.

Appearance: colorless oil.

TLC: $R_{f}=0.7$ (pentane/EtOAc = 19:1, UV-active and stains blue in PMA or in vanillin).

${ }^{1} \mathrm{H}-\mathrm{NMR}\left(400 \mathrm{MHz}, \mathrm{CDCl}_{3}\right) \delta(\mathrm{ppm})=7.40-7.29(\mathrm{~m}, 5 \mathrm{H}), 5.11(\mathrm{~s}, 2 \mathrm{H}), 2.35-2.28(\mathrm{~m}, 2 \mathrm{H}), 1.94$ $(p, J=3.0 \mathrm{~Hz}, 3 \mathrm{H}), 1.73-1.66(\mathrm{~m}, 3 \mathrm{H}), 1.64-1.58(\mathrm{~m}, 3 \mathrm{H}), 1.49-1.41(\mathrm{~m}, 8 \mathrm{H})$.

${ }^{13} \mathrm{C}-\mathrm{NMR}\left(101 \mathrm{MHz}, \mathrm{CDCl}_{3}\right) \delta(\mathrm{ppm})=174.5,136.1,128.5,128.2,128.1,66.1,42.0,38.9,37.1$, 31.9, 28.6, 28.2.

HRMS (ESI-TOF) calc'd for $\mathrm{C}_{20} \mathrm{H}_{26} \mathrm{O}_{2} \mathrm{Na}[\mathrm{M}+\mathrm{Na}]^{+}: 321.1825$, found: 321.1826 .

Synthesis of tert-butyl 4-(3-(benzyloxy)-3-oxopropyl)-4-methylpiperidine-1-carboxylate (4k)<smiles>[Y4]C1(C(=O)ON2C(=O)c3ccccc3C2=O)CCN(C(=O)OC(C)(C)C)CC1</smiles>

$2 \mathbf{k}$

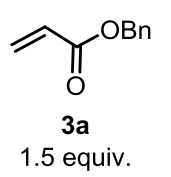

Conditions $A / B / C$

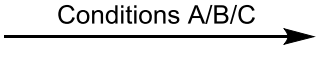

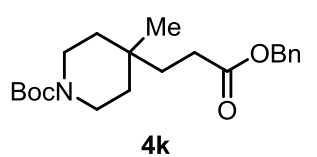

4k

General procedure B1 was applied using 1-(tert-butyl) 4-(1,3-dioxoisoindolin-2-yl) 4methylpiperidine-1,4-dicarboxylate (2k; $1.94 \mathrm{mg}, 5.0 \mu \mathrm{mol}, 1.0$ equiv.), benzyl acrylate (3a; $1.21 \mathrm{mg}, 7.5 \mu \mathrm{mol}, 1.5$ equiv.), 1-butyl-1,4-dihydropyridine-3-carboxamide (10; $2.71 \mathrm{mg}$, $0.015 \mathrm{mmol}, 3.0$ equiv.) in a mixture of DMSO $(2.5 \mathrm{~mL})$ and water $(2.5 \mathrm{~mL})$ for $60 \mathrm{~min}$ under 
argon. The crude yield was measured by ${ }^{1} \mathrm{H}-\mathrm{NMR}$ in presence of 1,1,2,2-tetrachloroethane to obtain $\mathbf{4 k}$ in $99 \%$.

General procedure B2 was applied using 1-(tert-butyl) 4-(1,3-dioxoisoindolin-2-yl) 4methylpiperidine-1,4-dicarboxylate (2k; $1.94 \mathrm{mg}, 5.0 \mu \mathrm{mol}, 1.0$ equiv.), benzyl acrylate (3a; $1.21 \mathrm{mg}, 7.5$ umol, 1.5 equiv.), NADH (11; $35.0 \mathrm{mg}, 0.05 \mathrm{mmol}, 10.0$ equiv.) in a mixture of DMSO $(2.5 \mathrm{~mL})$ and water $(2.5 \mathrm{~mL})$ for 60 min under argon. The crude yield was measured by ${ }^{1} \mathrm{H}$-NMR in presence of 1,1,2,2-tetrachloroethane to obtain $\mathbf{4 k}$ in $64 \%$.

General procedure B3 was applied using 1-(tert-butyl) 4-(1,3-dioxoisoindolin-2-yl) 4methylpiperidine-1,4-dicarboxylate ( $2 \mathbf{k} ; 58.3 \mathrm{mg}, 0.15 \mathrm{mmol}, 1.0$ equiv.), benzyl acrylate (3a; $36.5 \mathrm{mg}, 0.26 \mathrm{mmol}, 1.5$ equiv.), and $\mathrm{NADH}(11 ; 159 \mathrm{mg}, 0.26 \mathrm{mmol}, 1.5$ equiv.) in anhydrous DMSO $(1.5 \mathrm{~mL})$ for $30 \mathrm{~min}$ at $20{ }^{\circ} \mathrm{C}$ under argon. The crude was purified by column chromatography on $\mathrm{SiO}_{2}$ (pentane/EtOAc $=19: 1$ to $10: 1$ ) to afford $\mathbf{4 k}$ ( $29 \mathrm{mg}, 80 \mu \mathrm{mol}, 53 \%$ ).

Appearance: colorless oil.

TLC: $R_{f}=0.3$ (pentane/EtOAC $=10: 1$, UV-active and stains blue in vanillin).

${ }^{1} \mathrm{H}-\mathrm{NMR}\left(400 \mathrm{MHz}, \mathrm{CDCl}_{3}\right) \delta(\mathrm{ppm})=7.40-7.30(\mathrm{~m}, 5 \mathrm{H}), 5.11(\mathrm{~s}, 2 \mathrm{H}), 3.56(\mathrm{~d}, J=14.4 \mathrm{~Hz}, 2 \mathrm{H})$, 3.17 (ddd, $J=13.4,9.1,4.0 \mathrm{~Hz}, 2 \mathrm{H}), 2.36-2.30(\mathrm{~m}, 2 \mathrm{H}), 1.67-1.62(\mathrm{~m}, 2 \mathrm{H}), 1.45(\mathrm{~s}, 9 \mathrm{H}), 1.38$ $-1.26(\mathrm{~m}, 4 \mathrm{H}), 0.91(\mathrm{~s}, 3 \mathrm{H})$.

${ }^{13} \mathrm{C}-\mathrm{NMR}\left(101 \mathrm{MHz}, \mathrm{CDCl}_{3}\right) \delta(\mathrm{ppm})=173.9,154.9,135.9,128.6,128.3,128.3,79.3,66.3,39.7$, $36.5,36.3,31.1,28.8,28.5,22.8$.

HRMS (ESI-TOF) calc'd for $\mathrm{C}_{21} \mathrm{H}_{31} \mathrm{NO}_{4} \mathrm{Na}[\mathrm{M}+\mathrm{Na}]^{+}$: 384.2147; found: 384.2145 .

Synthesis of ((2-(1-phenylcyclopropyl)ethyl)sulfonyl)benzene (4I)

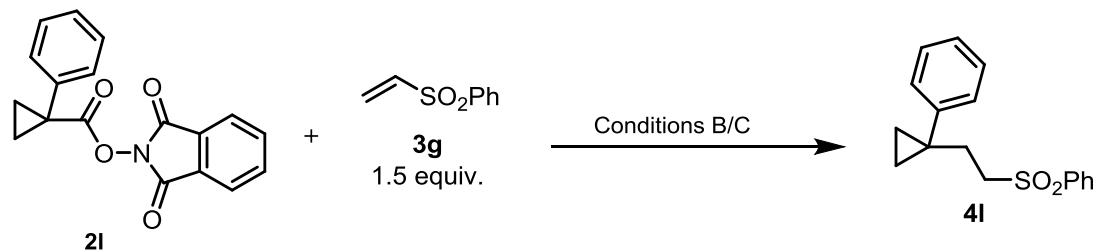

General procedure B2 was applied using 1,3-dioxoisoindolin-2-yl 1-phenylcyclopropane-1carboxylate (2l; $1.53 \mathrm{mg}, 5.0 \mu \mathrm{mol}, 1.0$ equiv.), phenyl vinyl sulfone (3g; $1.26 \mathrm{mg}, 7.5 \mu \mathrm{mol}$, 1.5 equiv.), NADH (11; $35.0 \mathrm{mg}, 0.05 \mathrm{mmol}, 10.0$ equiv.) in a mixture of DMSO (2.5 mL) and water $(2.5 \mathrm{~mL})$ for $60 \mathrm{~min}$ under argon. The crude yield was measured by ${ }^{1} \mathrm{H}-\mathrm{NMR}$ in presence of 1,1,2,2-tetrachloroethane to obtain 41 in $64 \%$.

General procedure B3 was applied using 1,3-dioxoisoindolin-2-yl 1-phenylcyclopropane-1carboxylate (2l; $92.8 \mathrm{mg}, 0.30 \mathrm{mmol}, 1.0$ equiv.), phenyl vinyl sulfone ( $3 \mathrm{~g} ; 75 \mathrm{mg}, 0.45 \mathrm{mmol}$, 1.5 equiv.), 1-butyl-1,4-dihydropyridine-3-carboxamide (10; $81 \mathrm{mg}, 0.45 \mathrm{mmol}, 1.5$ equiv.) in $3 \mathrm{~mL}$ DMSO for $5 \mathrm{~min}$. The crude product was purified by column chromatography on $\mathrm{SiO}_{2}$ (pentane/EtOAC = 19:1 to 9:1) to afford $4 \mathrm{l}$ ( $73 \mathrm{mg}, 0.25 \mathrm{mmol}, 85 \%$ ).

Appearance: colorless oil.

TLC: $R_{f}=0.5$ (pentane/EtOAc $=6: 1$, UV-active). 
${ }^{1} \mathrm{H}-\mathrm{NMR}\left(400 \mathrm{MHz}, \mathrm{CDCl}_{3}\right) \delta(\mathrm{ppm})=7.86-7.79(\mathrm{~m}, 2 \mathrm{H}), 7.67-7.60(\mathrm{~m}, 1 \mathrm{H}), 7.58-7.50(\mathrm{~m}$, $2 \mathrm{H}), 7.27-7.22(\mathrm{~m}, 2 \mathrm{H}), 7.20-7.13(\mathrm{~m}, 3 \mathrm{H}), 3.09-3.01(\mathrm{~m}, 2 \mathrm{H}), 2.00-1.93(\mathrm{~m}, 2 \mathrm{H}), 0.87-$ $0.82(\mathrm{~m}, 2 \mathrm{H}), 0.73-0.68(\mathrm{~m}, 2 \mathrm{H})$.

${ }^{13} \mathrm{C}-\mathrm{NMR}\left(100 \mathrm{MHz}, \mathrm{CDCl}_{3}\right) \delta(\mathrm{ppm})=142.8,139.2,133.6,129.2,128.6,128.5,127.9,126.6$, 54.4, 33.0, 24.4, 13.3 .

HRMS (ESI-TOF) calc'd for $\mathrm{C}_{17} \mathrm{H}_{18} \mathrm{O}_{2} \mathrm{SNa}[\mathrm{M}+\mathrm{Na}]^{+}: 309.0920$, found: 309.0927.

Synthesis of benzyl 7-(2,5-dimethylphenoxy)-4,4-dimethylheptanoate (4m)

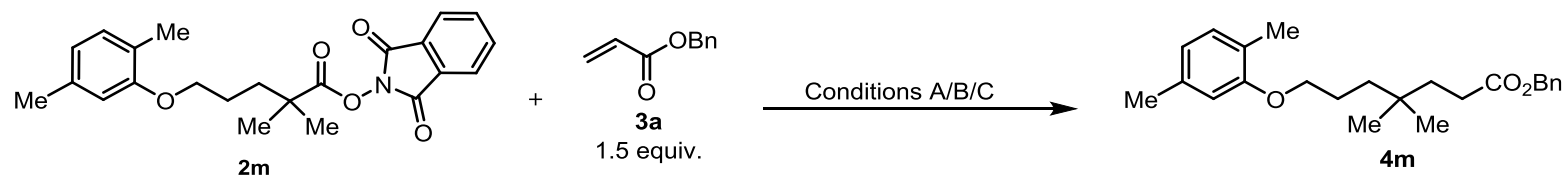

General procedure B1 was applied using 1,3-dioxoisoindolin-2-yl 5-(2,5-dimethylphenoxy)2,2-dimethylpentanoate ( $2 \mathrm{~m} ; 1.97 \mathrm{mg}, 5.0 \mu \mathrm{mol}, 1.0$ equiv.), benzyl acrylate (3a; $1.21 \mathrm{mg}, 7.5$ umol, 1.5 equiv.), 1-butyl-1,4-dihydropyridine-3-carboxamide (10; $2.71 \mathrm{mg}, 0.015 \mathrm{mmol}, 3.0$ equiv.) in a mixture of DMSO $(2.5 \mathrm{~mL})$ and water $(2.5 \mathrm{~mL})$ for 60 min under air. The crude yield was measured by ${ }^{1} \mathrm{H}-\mathrm{NMR}$ in presence of 1,1,2,2-tetrachloroethane to obtain $4 \mathrm{~m}$ in $95 \%$.

General procedure B2 was applied using 1,3-dioxoisoindolin-2-yl 5-(2,5-dimethylphenoxy)2,2-dimethylpentanoate ( $2 \mathrm{~m} ; 1.97 \mathrm{mg}, 5.0 \mu \mathrm{mol}, 1.0$ equiv.), benzyl acrylate (3a; $1.21 \mathrm{mg}, 7.5$ $\mu \mathrm{mol}, 1.5$ equiv.), NADH (11; $35.0 \mathrm{mg}, 0.05 \mathrm{mmol}, 10.0$ equiv.) in a mixture of DMSO ( $2.5 \mathrm{~mL})$ and water $(2.5 \mathrm{~mL})$ for 60 min under argon. The crude yield was measured by ${ }^{1} \mathrm{H}-\mathrm{NMR}$ in presence of 1,1,2,2-tetrachloroethane to obtain $4 \mathrm{~m}$ in $81 \%$.

General procedure B3 was applied using 1,3-dioxoisoindolin-2-yl 5-(2,5-dimethylphenoxy)2,2-dimethylpentanoate ( $2 \mathrm{~m} ; 118.6 \mathrm{mg}, 0.30 \mathrm{mmol}, 1.0$ equiv.), benzyl acrylate (3a; $73 \mathrm{mg}$, $0.45 \mathrm{mmol}, 1.5$ equiv.), 1-butyl-1,4-dihydropyridine-3-carboxamide (10; $81 \mathrm{mg}, 0.45 \mathrm{mmol}$, 1.5 equiv.) in $3 \mathrm{~mL}$ DMSO for $5 \mathrm{~min}$. The crude product was purified by column chromatography on $\mathrm{SiO}_{2}$ (pentane to pentane/EtOAc $=20: 1$ ) to afford $4 \mathrm{~m}(103 \mathrm{mg}, 0.28$ $\mathrm{mmol}, 93 \%)$.

Appearance: colorless oil.

TLC: $R_{f}=0.55$ (pentane/EtOAC $=20: 1$, UV-active and stains red in vanillin).

${ }^{1} \mathrm{H}-\mathrm{NMR}\left(400 \mathrm{MHz}, \mathrm{CDCl}_{3}\right) \delta(\mathrm{ppm})=7.40-7.30(\mathrm{~m}, 5 \mathrm{H}), 7.01(\mathrm{~d}, J=8.0 \mathrm{~Hz}, 1 \mathrm{H}), 6.67(\mathrm{~d}, J=$ $8.0 \mathrm{~Hz}, 1 \mathrm{H}), 6.62(\mathrm{~s}, 1 \mathrm{H}), 5.12(\mathrm{~s}, 2 \mathrm{H}), 3.91(\mathrm{t}, J=6.4 \mathrm{~Hz}, 2 \mathrm{H}), 2.39-2.33(\mathrm{~m}, 2 \mathrm{H}), 2.31(\mathrm{~s}, 3 \mathrm{H})$, $2.17(\mathrm{~s}, 3 \mathrm{H}), 1.79-1.70(\mathrm{~m}, 2 \mathrm{H}), 1.65-1.59(\mathrm{~m}, 2 \mathrm{H}), 1.40-1.32(\mathrm{~m}, 2 \mathrm{H}), 0.91(\mathrm{~s}, 6 \mathrm{H})$.

${ }^{13} \mathrm{C}-\mathrm{NMR}\left(101 \mathrm{MHz}, \mathrm{CDCl}_{3}\right) \delta(\mathrm{ppm})=174.1,157.0,136.4,136.1,130.3,128.6,128.2,128.2$, 123.6, 120.6, 112.0, 68.4, 66.2, 37.9, 36.3, 32.3, 29.6, 26.8, 24.2, 21.4, 15.8.

All the data are in accordance with the literature. ${ }^{[10]}$ 
Synthesis of 1-(7-(2,5-dimethylphenoxy)-4,4-dimethylheptanoyl)piperidine-4-carboxylic acid (4n)

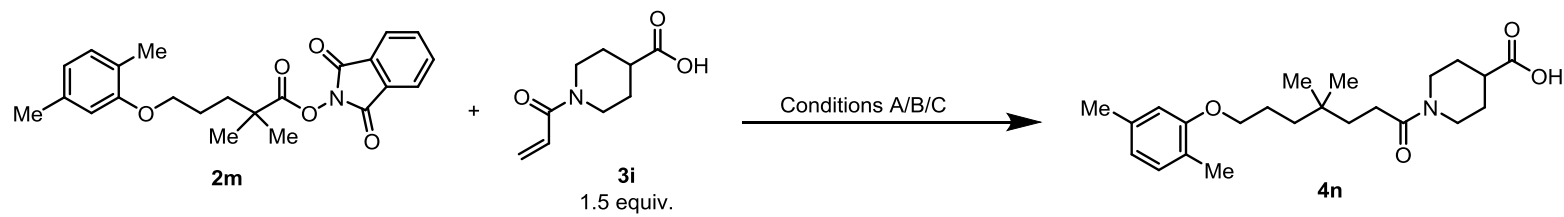

General procedure B1 was applied using 1,3-dioxoisoindolin-2-yl 5-(2,5-dimethylphenoxy)2,2-dimethylpentanoate (2m; $1.97 \mathrm{mg}, 5.0 \mu \mathrm{mol}, 1.0$ equiv.), 1-acryloylpiperidine-4carboxylic acid (3i; $1.37 \mathrm{mg}, 7.5 \mu \mathrm{mol}, 1.5$ equiv.), 1-butyl-1,4-dihydropyridine-3-carboxamide (10; $2.71 \mathrm{mg}, 0.015 \mathrm{mmol}, 3.0$ equiv.) in a mixture of DMSO $(2.5 \mathrm{~mL})$ and water $(2.5 \mathrm{~mL})$ for 60 min under argon. The crude yield was measured by ${ }^{1} \mathrm{H}-\mathrm{NMR}$ in presence of $1,1,2,2-$ tetrachloroethane to obtain $4 \mathrm{n}$ in $88 \%$.

General procedure B2 was applied using 1,3-dioxoisoindolin-2-yl 5-(2,5-dimethylphenoxy)2,2-dimethylpentanoate ( $2 \mathrm{~m} ; 1.97 \mathrm{mg}, 5.0 \mu \mathrm{mol}, 1.0$ equiv.), 1-acryloylpiperidine-4carboxylic acid (3i; $1.37 \mathrm{mg}, 7.5 \mu \mathrm{mol}, 1.5$ equiv.), NADH (11; $35.0 \mathrm{mg}, 0.05 \mathrm{mmol}, 10.0$ equiv.) in a mixture of DMSO $(2.5 \mathrm{~mL})$ and water $(2.5 \mathrm{~mL})$ for 60 min under argon. The crude yield was measured by ${ }^{1} \mathrm{H}-\mathrm{NMR}$ in presence of 1,1,2,2-tetrachloroethane to obtain $4 \mathbf{n}$ in $50 \%$.

General procedure B3 was applied using 1,3-dioxoisoindolin-2-yl 5-(2,5-dimethylphenoxy)2,2-dimethylpentanoate (2m; $59.0 \mathrm{mg}, 0.15 \mathrm{mmol}, 1.0$ equiv.), 1-acryloylpiperidine-4carboxylic acid (3i; $41.0 \mathrm{mg}, 0.23 \mathrm{mmol}, 1.5$ equiv.), 1-butyl-1,4-dihydropyridine-3carboxamide (10; $41.0 \mathrm{mg}, 0.23 \mathrm{mmol}, 1.5$ equiv.) in DMSO (1.5 mL) for $1 \mathrm{~h}$. The crude product was purified by column chromatography on $\mathrm{SiO}_{2}$ (pentane/EtOAc $=3: 2$ to $3: 7+1 \% \mathrm{AcOH}$ ) to afford 4n (45 mg, $0.12 \mathrm{mmol}, 77 \%$ ).

Appearance: colorless oil.

TLC: $\mathrm{Rf}=0.27$ (Pentane/EtOAC $=1: 1+1 \% \mathrm{AcOH}, \mathrm{UV}$-active and stains in PMA).

${ }^{1} \mathrm{H} \mathrm{MR}\left(400 \mathrm{MHz}, \mathrm{CDCl}_{3}\right) \delta(\mathrm{ppm})=7.00(\mathrm{~d}, J=7.4 \mathrm{~Hz}, 1 \mathrm{H}), 6.65(\mathrm{~d}, J=7.6 \mathrm{~Hz}, 1 \mathrm{H}), 6.62(\mathrm{~s}, 1 \mathrm{H})$, $4.47-4.34(\mathrm{~m}, 1 \mathrm{H}), 3.93(\mathrm{t}, J=6.3 \mathrm{~Hz}, 2 \mathrm{H}), 3.82(\mathrm{~d}, J=13.7 \mathrm{~Hz}, 1 \mathrm{H}$ ), 3.12 (ddd, $J=14.0,11.1$, $2.9 \mathrm{~Hz}, 1 \mathrm{H}), 2.84(\mathrm{ddd}, J=13.9,11.0,3.0 \mathrm{~Hz}, 1 \mathrm{H}), 2.57(\mathrm{tt}, J=10.6,4.0 \mathrm{~Hz}, 1 \mathrm{H}), 2.36-2.26(\mathrm{~m}$, $5 \mathrm{H}), 2.17(\mathrm{~s}, 3 \mathrm{H}), 2.02-1.90(\mathrm{~m}, 2 \mathrm{H}), 1.84-1.62(\mathrm{~m}, 4 \mathrm{H}), 1.60-1.52(\mathrm{~m}, 2 \mathrm{H}), 1.43-1.34(\mathrm{~m}$, $2 \mathrm{H}), 0.92(\mathrm{~s}, 6 \mathrm{H})$.

${ }^{13} \mathrm{C}-\mathrm{NMR}\left(101 \mathrm{MHz}, \mathrm{CDCl}_{3}\right) \delta(\mathrm{ppm})=179.2,172.6,157.1,136.6,130.4,123.6,120.8,112.1$, 68.5, 45.1, 41.2, 40.8, 38.0, 37.1, 32.5, 28.6, 28.5, 27.8, 27.0, 24.4, 21.5, 15.9.

HRMS (ESI-TOF) calc'd for $\mathrm{C}_{23} \mathrm{H}_{34} \mathrm{NO}_{4}[\mathrm{M}-\mathrm{H}]^{-}: 388.2482$, found: 388.2489 .

Synthesis of benzyl 3-(1-benzoy/piperidin-4-yl)propanoate (4o)

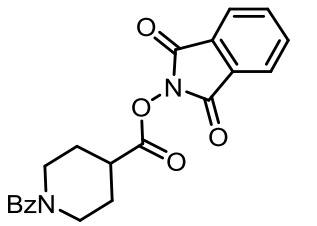

20

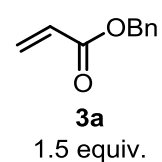

Conditions A/B/C

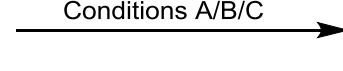

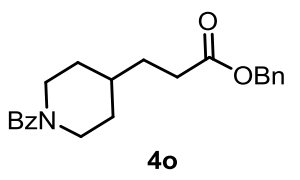

40 
General procedure B1 was applied using 1,3-dioxoisoindolin-2-yl 1-benzoylpiperidine-4carboxylate (2o; $1.89 \mathrm{mg}, 5.0 \mu \mathrm{mol}, 1.0$ equiv.), benzyl acrylate (3a; $1.21 \mathrm{mg}, 7.5 \mu \mathrm{mol}, 1.5$ equiv.), 1-butyl-1,4-dihydropyridine-3-carboxamide (10; $2.71 \mathrm{mg}, 0.015 \mathrm{mmol}, 3.0$ equiv.) in a mixture of DMSO $(2.5 \mathrm{~mL})$ and water $(2.5 \mathrm{~mL})$ for 60 min under air. The crude yield was measured by ${ }^{1} \mathrm{H}-\mathrm{NMR}$ in presence of 1,1,2,2-tetrachloroethane to obtain $4 \mathrm{o}$ in $90 \%$.

General procedure B2 was applied using 1,3-dioxoisoindolin-2-yl 1-benzoylpiperidine-4carboxylate (2o; $1.89 \mathrm{mg}, 5.0 \mu \mathrm{mol}, 1.0$ equiv.), benzyl acrylate (3a; $1.21 \mathrm{mg}, 7.5 \mu \mathrm{mol}, 1.5$ equiv.), NADH (11; $35.0 \mathrm{mg}, 0.05 \mathrm{mmol}, 10.0$ equiv.) in a mixture of DMSO $(2.5 \mathrm{~mL})$ and water $(2.5 \mathrm{~mL})$ for $60 \mathrm{~min}$ under argon. The crude yield was measured by ${ }^{1} \mathrm{H}-\mathrm{NMR}$ in presence of 1,1,2,2-tetrachloroethane to obtain 40 in $72 \%$.

General procedure B3 was applied using 1,3-dioxoisoindolin-2-yl 1-benzoylpiperidine-4carboxylate (2o; $98.2 \mathrm{mg}, 0.30 \mathrm{mmol}, 1.0$ equiv.), benzyl acrylate (3a; $73 \mathrm{mg}, 0.45 \mathrm{mmol}, 1.5$ equiv.), 1-butyl-1,4-dihydropyridine-3-carboxamide (10; $81 \mathrm{mg}, 0.45 \mathrm{mmol}, 1.5$ equiv.) in DMSO ( $3 \mathrm{~mL}$ ) for $5 \mathrm{~min}$. The crude product was purified by column chromatography on $\mathrm{SiO}_{2}$ (pentane/EtOAc $=8: 2$ to $6: 4)$ to afford $40(77 \mathrm{mg}, 0.22 \mathrm{mmol}, 73 \%){ }^{[10,11]}$

Appearance: colorless oil.

TLC: $R_{f}=0.45$ (pentane/EtOAc $=6: 4$, UV-active).

${ }^{1} \mathrm{H}-\mathrm{NMR}\left(400 \mathrm{MHz}, \mathrm{CDCl}_{3}\right) \delta(\mathrm{ppm})=7.41-7.30(\mathrm{~m}, 10 \mathrm{H}), 5.12(\mathrm{~s}, 2 \mathrm{H}), 4.69$ (br.s., $\left.1 \mathrm{H}\right), 3.72$ (br.s., $1 \mathrm{H}), 3.01-2.63(\mathrm{~m}, 2 \mathrm{H}), 2.39(\mathrm{t}, J=7.6 \mathrm{~Hz}, 2 \mathrm{H}), 1.86-1.46(\mathrm{~m}, 5 \mathrm{H}), 1.32-1.05(\mathrm{~m}, 2 \mathrm{H})$.

${ }^{13} \mathrm{C}-\mathrm{NMR}\left(101 \mathrm{MHz}, \mathrm{CDCl}_{3}\right) \delta(\mathrm{ppm})=173.3,170.3,136.4,135.9,129.4,128.6,128.6,128.4$, $128.3,126.8,66.3,47.9,42.3,35.6,31.5,31.2$.

HRMS (ESI-TOF) calc'd for $\mathrm{C}_{22} \mathrm{H}_{25} \mathrm{NO}_{3} \mathrm{Na}[\mathrm{M}+\mathrm{Na}]^{+}$: 374.1727; found: 374.1724 .

Synthesis of 2-(2-(phenylsulfonyl)ethyl)-2,3-dihydro-1H-indene (4p)

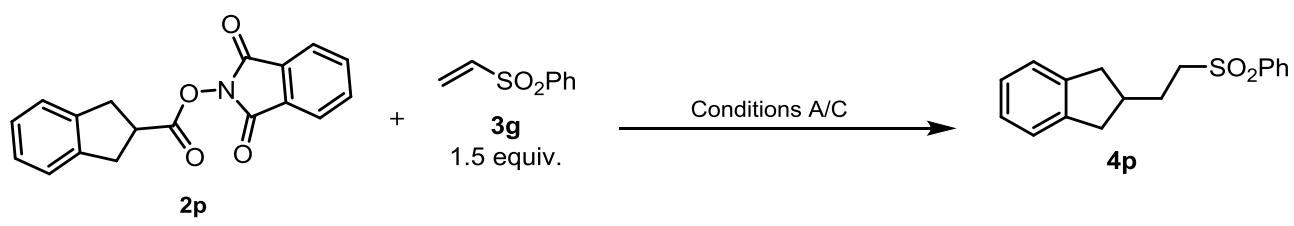

General procedure B1 was applied using 1,3-dioxoisoindolin-2-yl 2,3-dihydro-1H-indene-2carboxylate (2p; $1.53 \mathrm{mg}, 5.0 \mu \mathrm{mol}, 1.0$ equiv.), phenyl vinyl sulfone (3g; $1.26 \mathrm{mg}, 7.5 \mu \mathrm{mol}$, 1.5 equiv.), 1-butyl-1,4-dihydropyridine-3-carboxamide (10; $2.71 \mathrm{mg}, 0.015 \mathrm{mmol}, 3.0$ equiv.) in a mixture of DMSO $(2.5 \mathrm{~mL})$ and water $(2.5 \mathrm{~mL})$ for 60 min under air. The crude yield was measured by ${ }^{1} \mathrm{H}-\mathrm{NMR}$ in presence of 1,1,2,2-tetrachloroethane to obtain $4 \mathrm{p}$ in $61 \%$.

General procedure B3 was applied using 1,3-dioxoisoindolin-2-yl 2,3-dihydro-1H-indene-2carboxylate (2p; $92 \mathrm{mg}, 0.30 \mathrm{mmol}, 1.0$ equiv.), phenyl vinyl sulfone (3g; $76 \mathrm{mg}, 0.45 \mathrm{mmol}$, 1.5 equiv.), 1-butyl-1,4-dihydropyridine-3-carboxamide (10; $81 \mathrm{mg}, 0.45 \mathrm{mmol}, 1.5$ equiv.) in $3 \mathrm{~mL}$ DMSO for $5 \mathrm{~min}$. The crude product was purified by column chromatography on $\mathrm{SiO}_{2}$ (petroleum ether/EtOAc $=5: 1)$ to afford $4 p(45.0 \mathrm{mg}, 0.16 \mathrm{mmol}, 52 \%)$.

Appearance: white solid.

TLC: $R_{f}=0.50$ (petroleum ether $/$ EtOAc $=5: 1$, UV-active). 
${ }^{1} \mathrm{H}-\mathrm{NMR}\left(400 \mathrm{MHz}, \mathrm{CDCl}_{3}\right) \delta(\mathrm{ppm})=7.99-7.91(\mathrm{~m}, 2 \mathrm{H}), 7.74-7.66(\mathrm{~m}, 1 \mathrm{H}), 7.65-7.56(\mathrm{~m}$, $2 \mathrm{H}), 7.21-7.10(\mathrm{~m}, 4 \mathrm{H}), 3.23-3.15(\mathrm{~m}, 2 \mathrm{H}), 3.06(\mathrm{dd}, J=14.6,7.2 \mathrm{~Hz}, 2 \mathrm{H}), 2.63-2.46(\mathrm{~m}$, $3 \mathrm{H}), 2.02-1.90(\mathrm{~m}, 2 \mathrm{H})$.

${ }^{13} \mathrm{C}-\mathrm{NMR}\left(101 \mathrm{MHz}, \mathrm{CDCl}_{3}\right) \delta(\mathrm{ppm})=142.3,139.1,133.6,129.2,128.0,126.3,124.3,55.2$, 38.7, 38.6, 28.2.

All the data are in accordance with the literature. ${ }^{[10]}$

Synthesis of (1S,4S)-5-(2-(pheny/sulfonyl)ethyl)bicyclo[2.2.1]hept-2-ene (4q) and 3-(2(phenylsulfonyl)ethyl)tricyclo[2.2.1.02,6]heptane (4q')

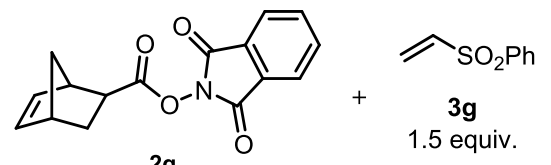

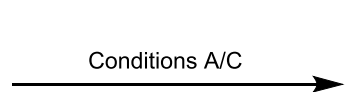

2q

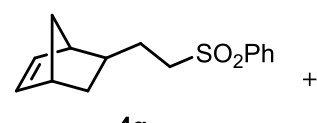

$4 q$

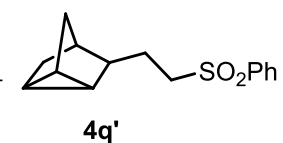

$4 q^{\prime}$

General procedure B1 was applied using 1,3-dioxoisoindolin-2-yl $(1 R, 4 R)$-bicyclo[2.2.1]hept5-ene-2-carboxylate (2q; $1.41 \mathrm{mg}, 5.0 \mu \mathrm{mol}, 1.0$ equiv.), phenyl vinyl sulfone (3g; $1.26 \mathrm{mg}, 7.5$ $\mu \mathrm{mol}, 1.5$ equiv.), and 1-butyl-1,4-dihydropyridine-3-carboxamide (10; $2.71 \mathrm{mg}, 0.015 \mathrm{mmol}$, 3.0 equiv.) in a mixture of DMSO $(2.5 \mathrm{~mL})$ and water $(2.5 \mathrm{~mL})$ for 60 min under air. The crude yield was measured by ${ }^{1} \mathrm{H}-\mathrm{NMR}$ in presence of $1,1,2,2$-tetrachloroethane to obtain $\mathbf{4} \mathbf{q}, \mathbf{q}^{\mathbf{\prime}}$ in $78 \%$ as a mixture (1.2:1).

General procedure B3 was applied using 1,3-dioxoisoindolin-2-yl $(1 R, 4 R)$-bicyclo[2.2.1]hept5-ene-2-carboxylate (2q; 85 mg, 0.30 mmol, 1.0 equiv.), phenyl vinyl sulfone (3g; 75 mg, 0.45 mmol, 1.5 equiv.), and 1-butyl-1,4-dihydropyridine-3-carboxamide (10; $81 \mathrm{mg}, 0.45 \mathrm{mmol}, 1.5$ equiv.) in DMSO ( $3 \mathrm{~mL}$ ) for $15 \mathrm{~min}$. The crude product was purified by column chromatography on $\mathrm{SiO}_{2}$ (pentane/EtOAc $=19: 1$ to 9:1) to afford $\mathbf{4 q}, \mathbf{q}^{\prime}(43 \mathrm{mg}, 0.16 \mathrm{mmol}, 57 \%$ ) as inseparable mixture (1.2:1).

Appearance: colorless oil.

TLC: $R_{f}=0.55$ (pentane/EtOAC $=6: 1, \mathrm{UV}$-active and stains in permanganate).

${ }^{1} \mathrm{H}-\mathrm{NMR}\left(400 \mathrm{MHz}, \mathrm{CDCl}_{3}\right) \delta(\mathrm{ppm})=7.94-7.87(\mathrm{~m}, 4 \mathrm{H}), 7.69-7.62(\mathrm{~m}, 2 \mathrm{H}), 7.61-7.54(\mathrm{~m}$, 4H), $6.02(\mathrm{dt}, J=2.5,1.1 \mathrm{~Hz}, 2 \mathrm{H} ; 4 \mathrm{q}), 3.17-3.00(\mathrm{~m}, 4 \mathrm{H}), 2.79(\mathrm{dq}, J=3.5,1.6 \mathrm{~Hz}, 1 \mathrm{H} ; 4 \mathrm{q}), 2.47$ $(\mathrm{dt}, J=3.0,1.4 \mathrm{~Hz}, 1 \mathrm{H} ; 4 \mathrm{q}), 1.87-1.70(\mathrm{~m}, 2 \mathrm{H}), 1.67-1.64\left(\mathrm{~m}, 1 \mathrm{H} ; 4 \mathrm{q}^{\prime}\right), 1.63-1.50(\mathrm{~m}, 2 \mathrm{H})$, 1.46 (qt, $\left.J=7.5,1.8 \mathrm{~Hz}, 1 \mathrm{H} ; \mathbf{4 q}^{\prime}\right), 1.37-1.18(\mathrm{~m}, 7 \mathrm{H}), 1.12-0.98(\mathrm{~m}, 4 \mathrm{H}), 0.86(\mathrm{tt}, J=5.2,1.2$ $\left.\mathrm{Hz}, 1 \mathrm{H} ; 4 \mathrm{q}^{\prime}\right)$.

${ }^{13} \mathrm{C}-\mathrm{NMR}\left(101 \mathrm{MHz}, \mathrm{CDCl}_{3}\right) \delta(\mathrm{ppm})=139.3,139.2,136.6,136.3,133.6,133.6,129.3,129.2$, 128.0, 128.0, 55.8, 55.3, 46.1, 45.1, 43.8, 41.9, 37.8, 34.0, 32.7, 32.6, 28.9, 28.9, 22.7, 14.5, 11.5, 9.6.

HRMS (ESI-TOF) calc'd for $\mathrm{C}_{15} \mathrm{H}_{18} \mathrm{O}_{2} \mathrm{SNa}[\mathrm{M}+\mathrm{Na}]^{+}:$285.0920; found: 285.0929. 

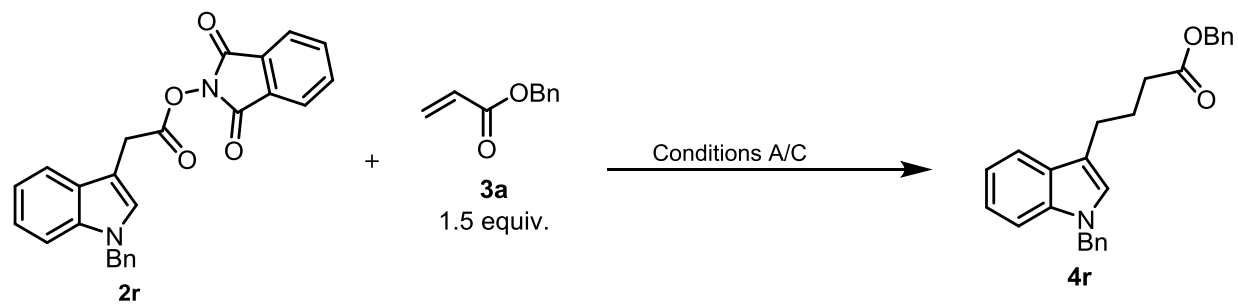

General procedure B1 was applied using 1,3-dioxoisoindolin-2-yl 2-(1-benzyl-1H-indol-3yl)acetate (2r; $2.05 \mathrm{mg}, 5.0 \mu \mathrm{mol}, 1.0$ equiv.), benzyl acrylate (3a; $1.21 \mathrm{mg}, 7.5 \mu \mathrm{mol}, 1.5$ equiv.), and 1-butyl-1,4-dihydropyridine-3-carboxamide (10; $2.71 \mathrm{mg}, 0.015 \mathrm{mmol}, 3.0$ equiv.) in a mixture of DMSO $(2.5 \mathrm{~mL})$ and water $(2.5 \mathrm{~mL})$ for 60 min under argon. The crude yield was measured by ${ }^{1} \mathrm{H}-\mathrm{NMR}$ in presence of 1,1,2,2-tetrachloroethane to obtain $4 \mathbf{r}$ in $50 \%$. The reaction was also performed in water $(1 \mathrm{mM})$, which gave rise to $51 \%{ }^{1} \mathrm{H}-\mathrm{NMR}$ yield of $4 \mathbf{r}$.

General procedure B3 was applied using 1,3-dioxoisoindolin-2-yl 2-(1-benzyl-1H-indol-3yl)acetate (2r; $123.1 \mathrm{mg}, 0.30 \mathrm{mmol}, 1.0$ equiv.), benzyl acrylate (3a; $73 \mathrm{mg}, 0.45 \mathrm{mmol}, 1.5$ equiv.), and 1-butyl-1,4-dihydropyridine-3-carboxamide (10; $81 \mathrm{mg}, 0.45 \mathrm{mmol}, 1.5$ equiv.) in DMSO ( $3 \mathrm{~mL}$ ) for $2 \mathrm{~h}$. The crude product was purified by column chromatography on $\mathrm{SiO}_{2}$ (pentane/EtOAc = 99:1 to 98:2) to afford $4 \mathrm{r}(35 \mathrm{mg}, 0.09 \mathrm{mmol}, 30 \%$ ).

Appearance: white solid.

TLC: $R_{f}=0.47$ (pentane/EtOAc $=19: 1$, UV-active and stains violet in vanillin).

m.p.: $40.1-43.1^{\circ} \mathrm{C}$.

${ }^{1} \mathrm{H}-\mathrm{NMR}\left(400 \mathrm{MHz}, \mathrm{CDCl}_{3}\right) \delta(\mathrm{ppm})=7.59(\mathrm{dd}, J=7.8,1.0 \mathrm{~Hz}, 1 \mathrm{H}), 7.39-7.30(\mathrm{~m}, 5 \mathrm{H}), 7.30-$ $7.22(\mathrm{~m}, 4 \mathrm{H}), 7.16$ (ddd, $J=8.3,7.0,1.3 \mathrm{~Hz}, 1 \mathrm{H}), 7.13-7.06(\mathrm{~m}, 3 \mathrm{H}), 6.88(\mathrm{~s}, 1 \mathrm{H}), 5.26(\mathrm{~s}, 2 \mathrm{H})$, $5.11(\mathrm{~s}, 2 \mathrm{H}), 2.81(\mathrm{td}, J=7.5,0.9 \mathrm{~Hz}, 2 \mathrm{H}), 2.44(\mathrm{t}, J=7.4 \mathrm{~Hz}, 2 \mathrm{H}), 2.07(\mathrm{p}, J=7.5 \mathrm{~Hz}, 2 \mathrm{H})$.

${ }^{13} \mathrm{C}-\mathrm{NMR}\left(101 \mathrm{MHz}, \mathrm{CDCl}_{3}\right) \delta(\mathrm{ppm})=173.5,137.7,136.7,136.1,128.7,128.5,128.2,128.2$, $128.1,127.5,126.8,125.7,121.7,119.1,118.9,114.8,109.6,66.1,49.9,33.9,25.5,24.4$.

HRMS (ESI-TOF) calc'd for $\mathrm{C}_{26} \mathrm{H}_{25} \mathrm{NO}_{2} \mathrm{Na}[\mathrm{M}+\mathrm{Na}]^{+}$: 406.1778; found: 406.1772 .

Synthesis of biotin-glucosamine conjugate (4s)
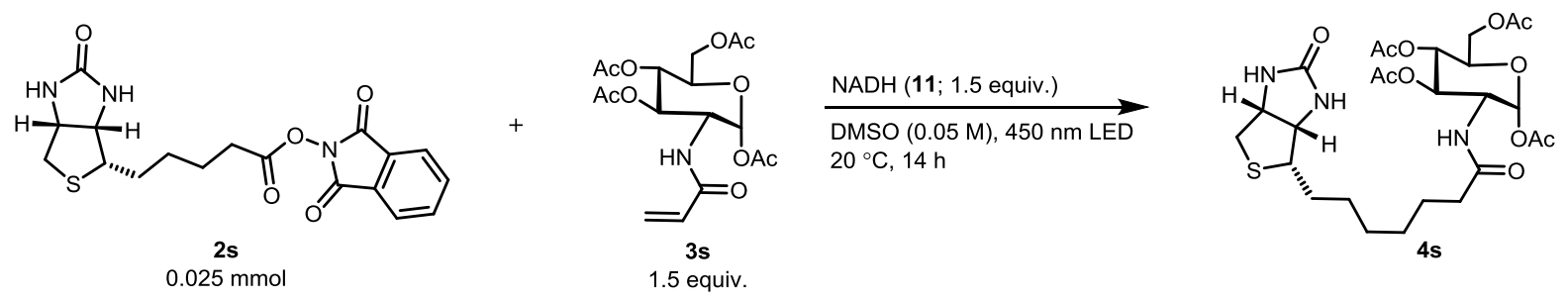

Biotin NHPI-ester (2s; 9.7 mg, $25.0 \mu \mathrm{mol}, 1.0$ equiv.), $\mathrm{N}$-acroloyl glucosamine derivative (3s; $15.1 \mathrm{mg}, 38.0 \mu \mathrm{mol}, 1.5$ equiv.), and $\mathrm{NADH}$ (11; $26.6 \mathrm{mg}, 38.0 \mu \mathrm{mol}, 1.5$ equiv.) were carefully added in a vial. The vial was then evacuated and refilled with argon (three cycles). DMSO (0.5 $\mathrm{mL}$ ) was added to the vial. The mixture was irradiated with blue light for $14 \mathrm{~h}$. Upon completion, water $(10 \mathrm{~mL})$ and $\mathrm{CHCl}_{3}(10 \mathrm{~mL})$ were added to the reaction mixture. The organic layer was separated and the aqueous layer was extracted with $\mathrm{CHCl}_{3}(2 \times 10 \mathrm{~mL})$. The combined organic layer was washed with brine $(10 \mathrm{~mL})$, dried over $\mathrm{Na}_{2} \mathrm{SO}_{4}$, and concentrated under 
reduced pressure. The crude was purified by column chromatography on $\mathrm{SiO}_{2}$ (DCM/methanol = 99:1 to 9:1) followed by recrystallization with $\mathrm{DCM} /$ petroleum ether to afford 4s.

${ }^{1} \mathrm{H}-\mathrm{NMR}$ yield: $49 \%$

Appearance: white solid.

TLC: $R_{f}=0.5$ (DCM/methanol = 9:1, stains in permanganate) .

m.p.: $133.4-137.6^{\circ} \mathrm{C}$.

${ }^{1} \mathrm{H}-\mathrm{NMR}\left(400 \mathrm{MHz}^{\mathrm{CDCl}}{ }_{3}\right) \delta(\mathrm{ppm})=6.60(\mathrm{~d}, J=9.6 \mathrm{~Hz}, 1 \mathrm{H}), 5.99(\mathrm{~s}, 1 \mathrm{H}), 5.73(\mathrm{~d}, J=8.8 \mathrm{~Hz}$, $1 \mathrm{H}), 5.31-5.09(\mathrm{~m}, 3 \mathrm{H}), 4.54(\mathrm{dd}, J=7.8,5.0 \mathrm{~Hz}, 1 \mathrm{H}), 4.41-4.24(\mathrm{~m}, 3 \mathrm{H}), 4.11(\mathrm{dd}, J=12.5$, $2.2 \mathrm{~Hz}, 1 \mathrm{H}$ ), 3.82 (ddd, $J=9.5,4.6,2.2 \mathrm{~Hz}, 1 \mathrm{H}), 3.16$ (q, $J=7.1,6.2 \mathrm{~Hz}, 1 \mathrm{H}), 2.94$ (dd, $J=12.8$, $5.0 \mathrm{~Hz}, 1 \mathrm{H}), 2.74(\mathrm{~d}, J=12.6 \mathrm{~Hz}, 1 \mathrm{H}), 2.19-1.97(\mathrm{~m}, 14 \mathrm{H}), 1.76-1.68(\mathrm{~m}, 1 \mathrm{H}), 1.45-1.19(\mathrm{~m}$, $7 \mathrm{H})$.

${ }^{13} \mathrm{C}-\mathrm{NMR}\left(101 \mathrm{MHz}, \mathrm{CDCl}_{3}\right) \delta(\mathrm{ppm})=173.5,171.3,170.7,169.6,169.3,163.6,92.7,73.0,72.7$, 67.9, 62.0, 61.7, 60.2, 55.6, 52.6, 40.4, 36.6, 28.9, 28.8, 28.6, 28.4, 25.6, 21.0, 20.8, 20.8, 20.6.

HRMS (ESI-TOF) calc'd for $\mathrm{C}_{26} \mathrm{H}_{39} \mathrm{~N}_{3} \mathrm{O}_{11} \mathrm{SNa}[\mathrm{M}+\mathrm{Na}]^{+}: 624.2198$; found: 624.2199.

Synthesis of benzyl (Z)-icos-11-enoate (4t)
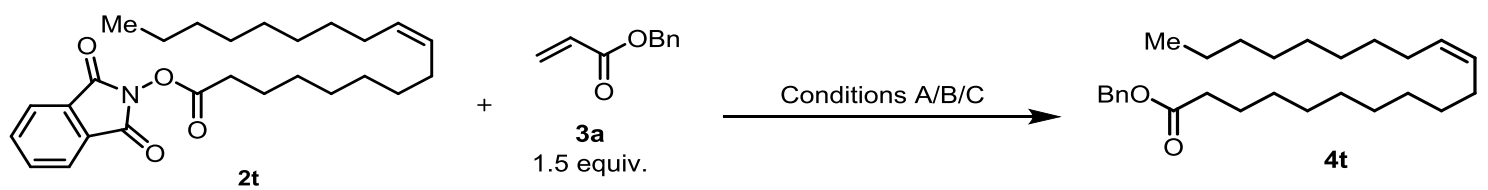

General procedure B1 was applied using 1,3-dioxoisoindolin-2-yl 1-benzoylpiperidine-4carboxylate (2t; $2.13 \mathrm{mg}, 5.0 \mu \mathrm{mol}, 1.0$ equiv.), benzyl acrylate (3a; $1.21 \mathrm{mg}, 7.5 \mu \mathrm{mol}, 1.5$ equiv.), 1-butyl-1,4-dihydropyridine-3-carboxamide (10; $2.71 \mathrm{mg}, 0.015 \mathrm{mmol}, 3.0$ equiv.) in DMSO ( $5 \mathrm{~mL}$ ) for $60 \mathrm{~min}$ under argon. The crude yield was measured by ${ }^{1} \mathrm{H}-\mathrm{NMR}$ in presence of 1,1,2,2-tetrachloroethane to obtain $4 \mathrm{t}$ in $61 \%$.

General procedure B2 was applied using 1,3-dioxoisoindolin-2-yl 1-benzoylpiperidine-4carboxylate (2t; $2.13 \mathrm{mg}, 5.0 \mu \mathrm{mol}, 1.0$ equiv.), benzyl acrylate (3a; $1.21 \mathrm{mg}, 7.5 \mu \mathrm{mol}, 1.5$ equiv.), NADH (11; $35.0 \mathrm{mg}, 0.05 \mathrm{mmol}, 10.0$ equiv.) in DMSO (0.25 mL) for $75 \mathrm{~min}$ under argon. The crude yield was measured by ${ }^{1} \mathrm{H}-\mathrm{NMR}$ in presence of 1,1,2,2-tetrachloroethane to obtain $4 t$ in $64 \%$.

General procedure B3 was applied using 1,3-dioxoisoindolin-2-yl oleate (2t; $128 \mathrm{mg}, 0.30$ mmol, 1.0 equiv.), benzyl acrylate (3a; $73 \mathrm{mg}, 0.45 \mathrm{mmol}, 1.5$ equiv.), 1-butyl-1,4dihydropyridine-3-carboxamide (10; $81 \mathrm{mg}, 0.45 \mathrm{mmol}, 1.5$ equiv.) in DMSO (3 mL) for $5 \mathrm{~min}$. The crude product was purified by column chromatography on $\mathrm{SiO}_{2}$ (petroleum ether/EtOAc $=20: 1)$ to afford $4 \mathrm{t}$ ( $85.0 \mathrm{mg}, 0.21 \mathrm{mmol}, 71 \%)$.

Appearance: colorless oil.

TLC: $R_{f}=0.41$ (petroleum ether/EtOAc $=10: 1$, UV-active and stains in PMA).

${ }^{1} \mathrm{H}-\mathrm{NMR}\left(\mathbf{4 0 0} \mathrm{MHz}, \mathrm{CDCl}_{3}\right) \delta(\mathrm{ppm})=7.39-7.30(\mathrm{~m}, 5 \mathrm{H}), 5.39-5.30(\mathrm{~m}, 2 \mathrm{H}), 5.14(\mathrm{~s}, 2 \mathrm{H}), 2.38$ $(\mathrm{t}, J=7.5 \mathrm{~Hz}, 2 \mathrm{H}), 2.12-1.97(\mathrm{~m}, 4 \mathrm{H}), 1.64(\mathrm{q}, J=7.3 \mathrm{~Hz}, 1 \mathrm{H}), 1.40-1.24(\mathrm{~m}, 24 \mathrm{H}), 0.94-0.87$ $(\mathrm{m}, 3 \mathrm{H})$. 
${ }^{13} \mathrm{C}-\mathrm{NMR}\left(101 \mathrm{MHz}, \mathrm{CDCl}_{3}\right) \delta(\mathrm{ppm})=173.6,136.1,129.9,129.8,128.5,128.1,128.1,66.0$, $34.3,31.9,29.7,29.7,29.5,29.4,29.4,29.3,29.2,29.2,29.1,27.2,24.9,22.6,14.0$.

All the data are in accordance with the literature. ${ }^{[10]}$

Synthesis of 3-(4-(phenylsulfonyl)butyl)pyridine (4u)
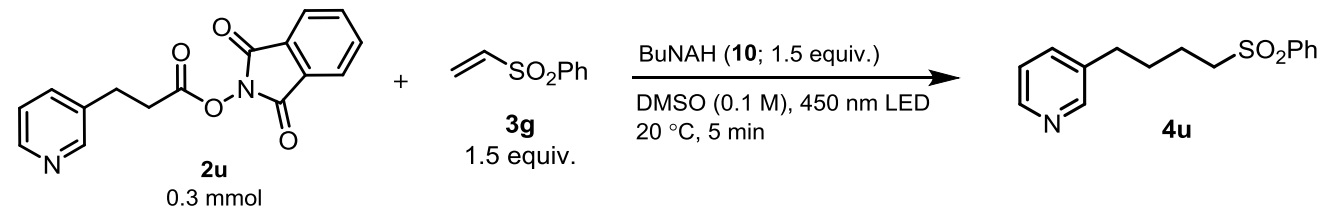

General procedure B3 was applied using 1,3-dioxoisoindolin-2-yl 3-(pyridin-3-yl)propanoate (2u; $89 \mathrm{mg}, 0.30 \mathrm{mmol}, 1.0$ equiv.), phenyl vinyl sulfone (3g; $76 \mathrm{mg}, 0.45 \mathrm{mmol}, 1.5$ equiv.), 1 butyl-1,4-dihydropyridine-3-carboxamide (10; $0.45 \mathrm{mmol}, 81 \mathrm{mg}, 1.5$ equiv.) in DMSO (3 mL) for $5 \mathrm{~min}$. The crude product was purified by column chromatography on $\mathrm{SiO}_{2}$ (petroleum ether $/$ EtOAc $=1: 1)$ to afford $\mathbf{4 u}(44.0 \mathrm{mg}, 0.16 \mathrm{mmol}, 53 \%)$.

Appearance: yellow oil.

TLC: $R_{f}=0.15$ (petroleum ether/EtOAC $=1: 1$, UV-active).

${ }^{1} \mathrm{H}$-NMR (400 MHz, CDCl $\left.{ }_{3}\right) \delta(\mathrm{ppm})=8.44(\mathrm{dd}, \mathrm{J}=4.8,1.7 \mathrm{~Hz}, 1 \mathrm{H}), 8.39(\mathrm{~d}, \mathrm{~J}=2.3 \mathrm{~Hz}, 1 \mathrm{H}), 7.97$ $-7.83(\mathrm{~m}, 2 \mathrm{H}), 7.71-7.63(\mathrm{~m}, 1 \mathrm{H}), 7.61-7.53(\mathrm{~m}, 2 \mathrm{H}), 7.45(\mathrm{dt}, \mathrm{J}=7.8,2.0 \mathrm{~Hz}, 1 \mathrm{H}), 7.21$ (ddd, $\mathrm{J}=7.8,4.8,0.9 \mathrm{~Hz}, 1 \mathrm{H}), 3.20-2.98(\mathrm{~m}, 2 \mathrm{H}), 2.61(\mathrm{t}, \mathrm{J}=7.3 \mathrm{~Hz}, 2 \mathrm{H}), 1.87-1.64(\mathrm{~m}, 4 \mathrm{H})$.

${ }^{13} \mathrm{C}-\mathrm{NMR}\left(101 \mathrm{MHz}, \mathrm{CDCl}_{3}\right) \delta(\mathrm{ppm})=149.7,147.6,139.2,136.6,135.9,133.8,129.4,128.1$, $123.5,56.0,32.5,29.7,22.3$.

HRMS (ESI-TOF) calc'd for $\mathrm{C}_{15} \mathrm{H}_{17} \mathrm{NO}_{2} \mathrm{SH}[\mathrm{M}+\mathrm{H}]^{+}:$276.1053; found: 276.1055 .

Synthesis of derivative of abietic acid (4v)
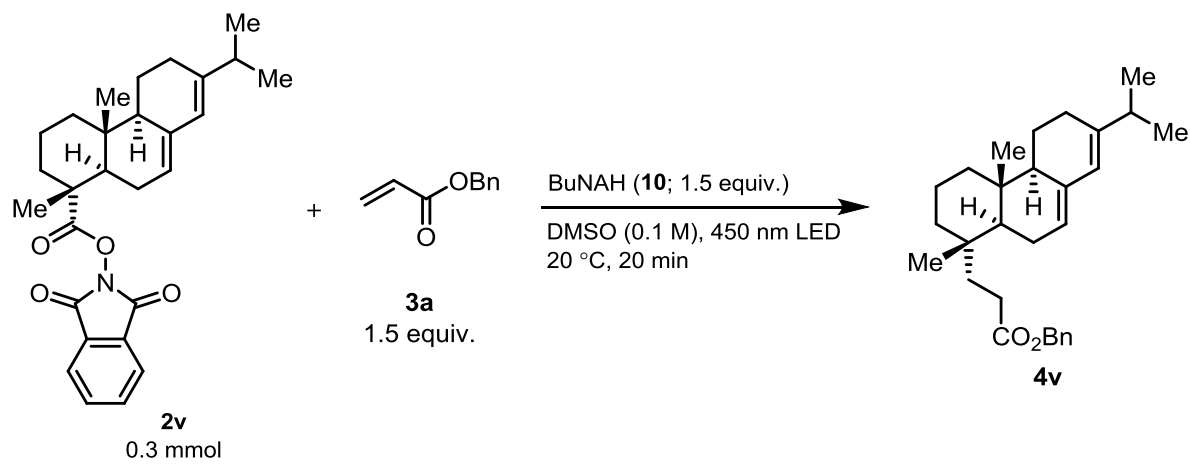

General procedure B3 was applied using redox-active ester of abietic acid (2v; $134.3 \mathrm{mg}, 0.30$ mmol, 1.0 equiv.), benzyl acrylate (3a; $73 \mathrm{mg}, 0.45 \mathrm{mmol}, 1.5$ equiv.), 1-butyl-1,4dihydropyridine-3-carboxamide (10; $81 \mathrm{mg}, 0.45 \mathrm{mmol}, 1.5$ equiv.) in DMSO (3 mL) for $20 \mathrm{~min}$. The crude product was purified by column chromatography on $\mathrm{SiO}_{2}$ (pentane to pentane/EtOAc $=99: 1)$ to afford $4 \mathbf{v}(87 \mathrm{mg}, 0.20 \mathrm{mmol}, 69 \%)$ with d.r. $>$ 20:1.

Appearance: colorless oil.

TLC: $R_{f}=0.64$ (pentane/EtOAc $=98: 2$, UV-active and stains green in vanillin). 
${ }^{1} \mathrm{H}-\mathrm{NMR}\left(400 \mathrm{MHz}, \mathrm{CDCl}_{3}\right) \delta(\mathrm{ppm})=7.40-7.29(\mathrm{~m}, 5 \mathrm{H}), 5.78(\mathrm{~s}, 1 \mathrm{H}), 5.40(\mathrm{dt}, J=5.2,2.4 \mathrm{~Hz}$, $1 \mathrm{H}), 5.09(\mathrm{~s}, 2 \mathrm{H}), 2.30-2.19(\mathrm{~m}, 3 \mathrm{H}), 2.13-2.04(\mathrm{~m}, 3 \mathrm{H}), 2.01-1.94(\mathrm{~m}, 1 \mathrm{H}), 1.88-1.76(\mathrm{~m}$, $3 \mathrm{H}), 1.66-1.45(\mathrm{~m}, 5 \mathrm{H}), 1.36(\mathrm{dtd}, J=12.8,3.2,1.6 \mathrm{~Hz}, 1 \mathrm{H}), 1.31-1.25(\mathrm{~m}, 1 \mathrm{H}), 1.24-1.14$ $(\mathrm{m}, 2 \mathrm{H}), 1.01$ (ddd, $J=7.2,3.7,1.8 \mathrm{~Hz}, 6 \mathrm{H}), 0.93(\mathrm{~s}, 3 \mathrm{H}), 0.82(\mathrm{~s}, 3 \mathrm{H})$.

${ }^{13} \mathrm{C}-\mathrm{NMR}\left(101 \mathrm{MHz}, \mathrm{CDCl}_{3}\right) \delta(\mathrm{ppm})=174.3,145.2,136.1,135.4,128.5,128.2,128.2,122.4$, 121.1, 66.2, 51.1, 47.9, 39.0, 38.6, 37.6, 35.1, 34.9, 34.9, 28.9, 27.5, 23.7, 22.7, 21.4, 20.8, 20.6, 18.5, 14.1.

HRMS (ESI-TOF) calc'd for $\mathrm{C}_{29} \mathrm{H}_{40} \mathrm{O}_{2} \mathrm{Na}[\mathrm{M}+\mathrm{Na}]^{+}: 443.2931$; found: 443.2930 .

Synthesis of benzyl 4-acetamidopentanoate (4w)

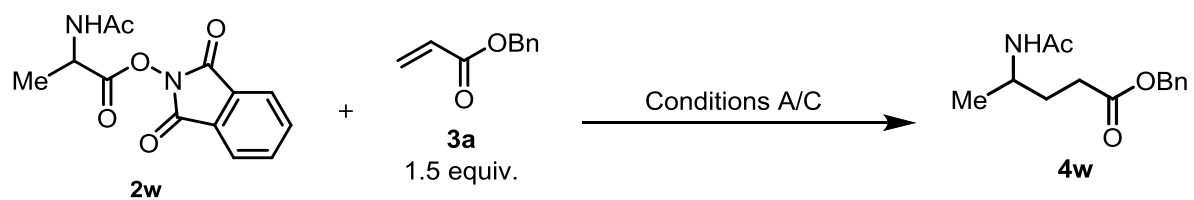

General procedure B1 was applied using 1,3-dioxoisoindolin-2-yl 2-(1-benzyl-1H-indol-3yl)acetate ( $2 \mathrm{w} ; 1.38 \mathrm{mg}, 5.0 \mu \mathrm{mol}, 1.0$ equiv.), benzyl acrylate (3a; $1.21 \mathrm{mg}, 7.5 \mu \mathrm{mol}, 1.5$ equiv.), and 1-butyl-1,4-dihydropyridine-3-carboxamide (10; $2.71 \mathrm{mg}, 0.015 \mathrm{mmol}, 3.0$ equiv.) in a mixture of DMSO $(2.5 \mathrm{~mL})$ and water $(2.5 \mathrm{~mL})$ for 60 min under air. The crude yield was measured by ${ }^{1} \mathrm{H}$-NMR in presence of $1,1,2,2$-tetrachloroethane to obtain $4 \mathbf{w}$ in $75 \%$. The reaction was also performed in water $(1 \mathrm{mM})$, which gave rise to $60 \%{ }^{1} \mathrm{H}-\mathrm{NMR}$ yield of $4 \mathbf{w}$.

General procedure B3 was applied using 1,3-dioxoisoindolin-2-yl acetylalaninate (2w; $83 \mathrm{mg}$, $0.30 \mathrm{mmol}, 1.0$ equiv.), benzyl acrylate (3a; $73 \mathrm{mg}, 0.45 \mathrm{mmol}, 1.5$ equiv.), 1-butyl-1,4dihydropyridine-3-carboxamide (10; $81 \mathrm{mg}, 0.45 \mathrm{mmol}, 1.5$ equiv.) in DMSO (3 mL) for $5 \mathrm{~min}$. The crude product was purified by preparative TLC (DCM/MeOH/Et $3 \mathrm{~N}=50: 1: 1)$ to afford $4 \mathbf{w}$ (27.0 mg, $0.11 \mathrm{mmol}, 36 \%$ ).

Appearance: white solid.

TLC: $R_{f}=0.20$ (petroleum ether/EtOAc $=1: 1$, UV-active and stains in iodine).

m.p.: $64.3-66.5^{\circ} \mathrm{C}$.

${ }^{1} \mathrm{H}-\mathrm{NMR}\left(400 \mathrm{MHz}, \mathrm{CDCl}_{3}\right) \delta(\mathrm{ppm})=7.45-7.29(\mathrm{~m}, 5 \mathrm{H}), 5.48-5.36(\mathrm{~m}, 1 \mathrm{H}), 5.19-5.09(\mathrm{~m}$, $2 \mathrm{H}), 4.11-3.94(\mathrm{~m}, 1 \mathrm{H}), 2.54-2.33(\mathrm{~m}, 2 \mathrm{H}), 1.92(\mathrm{~s}, 3 \mathrm{H}), 1.87-1.73(\mathrm{~m}, 2 \mathrm{H}), 1.17(\mathrm{~d}, J=6.6$ $\mathrm{Hz}, 3 \mathrm{H})$.

${ }^{13} \mathrm{C}-\mathrm{NMR}\left(101 \mathrm{MHz}, \mathrm{CDCl}_{3}\right) \delta(\mathrm{ppm})=173.6,169.6,136.0,128.7,128.4,128.3,66.5,45.3$, 31.6, 31.3, 23.5, 21.2.

HRMS (ESI-TOF) calc'd for $\mathrm{C}_{14} \mathrm{H}_{19} \mathrm{NO}_{3} \mathrm{Na}[\mathrm{M}+\mathrm{Na}]^{+}: 272.1257$; found: 272.1250 . 

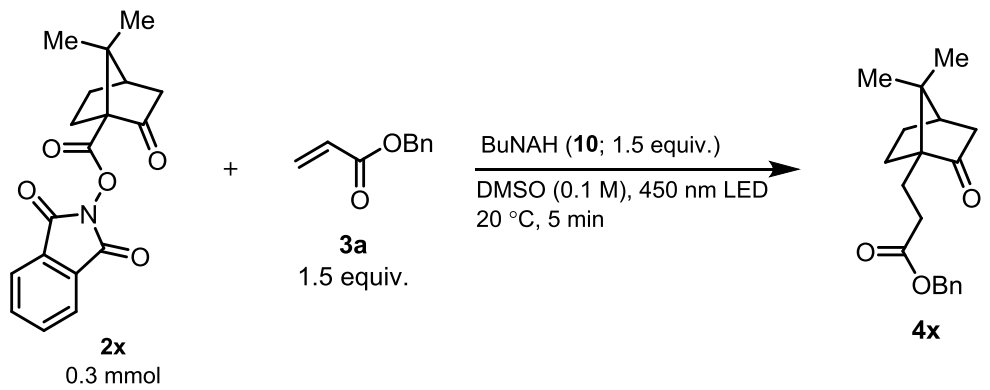

General procedure B3 was applied using 1,3-dioxoisoindolin-2-yl (1R,4R)-7,7-dimethyl-2oxobicyclo[2.2.1] heptane-1-carboxylate (2x; $98.2 \mathrm{mg}, 0.30 \mathrm{mmol}, 1.0$ equiv.), benzyl acrylate (3a; $73 \mathrm{mg}, 0.45 \mathrm{mmol}, 1.5$ equiv.), 1-butyl-1,4-dihydropyridine-3-carboxamide (10; $81 \mathrm{mg}$, $0.45 \mathrm{mmol}, 1.5$ equiv.) in DMSO ( $3 \mathrm{~mL}$ ) for $5 \mathrm{~min}$. The crude product was purified by column chromatography on $\mathrm{SiO}_{2}$ (pentane/EtOAc $=98: 2$ to $19: 1$ ) to afford $4 \mathbf{x}(51 \mathrm{mg}, 0.17 \mathrm{mmol}$, $56 \%)$.

Appearance: white solid.

TLC: $R_{f}=0.45$ (Pentane/EtOAc $=12: 1$, UV-active and stains blue in vanillin).

m.p.: $45.7-49.4^{\circ} \mathrm{C}$.

${ }^{1} \mathrm{H}-\mathrm{NMR}\left(400 \mathrm{MHz}, \mathrm{CDCl}_{3}\right) \delta(\mathrm{ppm})=7.40-7.29(\mathrm{~m}, 5 \mathrm{H}), 5.12(\mathrm{~s}, 2 \mathrm{H}), 2.88$ (ddd, $J=16.6,11.5$, $5.2 \mathrm{~Hz}, 1 \mathrm{H}), 2.39-2.27(\mathrm{~m}, 2 \mathrm{H}), 2.05(\mathrm{t}, J=4.5 \mathrm{~Hz}, 1 \mathrm{H}), 2.00-1.92(\mathrm{~m}, 1 \mathrm{H}), 1.92-1.85(\mathrm{~m}$, $1 \mathrm{H}), 1.81(\mathrm{~d}, J=18.3 \mathrm{~Hz}, 1 \mathrm{H}), 1.75-1.67(\mathrm{td}, J=16.0,4.0 \mathrm{~Hz}, 1 \mathrm{H}), 1.64-1.55(\mathrm{~m}, 1 \mathrm{H}), 1.45$ (ddd, $J=13.3,9.3,4.4 \mathrm{~Hz}, 1 \mathrm{H}$ ), 1.35 (ddd, $J=12.9,9.3,3.7 \mathrm{~Hz}, 1 \mathrm{H}), 0.96(\mathrm{~s}, 3 \mathrm{H}), 0.88(\mathrm{~s}, 3 \mathrm{H}$ ).

${ }^{13} \mathrm{C}-\mathrm{NMR}\left(101 \mathrm{MHz}, \mathrm{CDCl}_{3}\right) \delta(\mathrm{ppm})=218.9,173.9,136.1,128.5,128.3,128.2,66.2,59.2,47.5$, 43.4, 43.3, 29.8, 26.9, 26.7, 20.9, 20.2, 19.5.

HRMS (ESI-TOF) calc'd for $\mathrm{C}_{19} \mathrm{H}_{24} \mathrm{O}_{3} \mathrm{Na}[\mathrm{M}+\mathrm{Na}]^{+}$: 323.1618; found: 323.1620 .

Synthesis of derivative of oleanolic acid (4y)
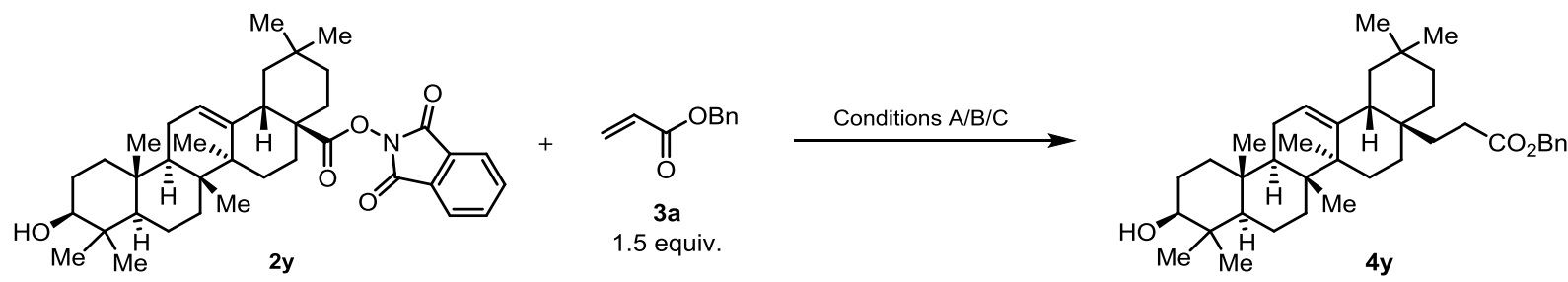

General procedure B1 was applied using 1,3-dioxoisoindolin-2-yl 1-benzoylpiperidine-4carboxylate (2y; $3.0 \mathrm{mg}, 5.0 \mu \mathrm{mol}, 1.0$ equiv.), benzyl acrylate (3a; $1.21 \mathrm{mg}, 7.5 \mu \mathrm{mol}, 1.5$ equiv.), 1-butyl-1,4-dihydropyridine-3-carboxamide (10; $2.71 \mathrm{mg}, 0.015 \mathrm{mmol}, 3.0$ equiv.) in DMSO ( $5 \mathrm{~mL}$ ) for $60 \mathrm{~min}$ under argon. The crude yield was measured by ${ }^{1} \mathrm{H}-\mathrm{NMR}$ in presence of 1,1,2,2-tetrachloroethane to obtain $4 \mathbf{y}$ in $74 \%$.

General procedure B2 was applied using 1,3-dioxoisoindolin-2-yl 1-benzoylpiperidine-4carboxylate (2y; $2.13 \mathrm{mg}, 5.0 \mu \mathrm{mol}, 1.0$ equiv.), benzyl acrylate (3a; $1.21 \mathrm{mg}, 7.5 \mu \mathrm{mol}, 1.5$ equiv.), NADH (11; $35.0 \mathrm{mg}, 0.05 \mathrm{mmol}, 10.0$ equiv.) in DMSO (0.02 M) for $75 \mathrm{~min}$ under argon. 
The crude yield was measured by ${ }^{1} \mathrm{H}-\mathrm{NMR}$ in presence of 1,1,2,2-tetrachloroethane to obtain $4 \mathbf{y}$ in $71 \%$.

General procedure B3 was applied using redox-active ester of oleanolic acid (2y; $180.5 \mathrm{mg}$, $0.30 \mathrm{mmol}, 1.0$ equiv.), benzyl acrylate (3a; $73 \mathrm{mg}, 0.45 \mathrm{mmol}, 1.5$ equiv.), 1-butyl-1,4dihydropyridine-3-carboxamide (10; $81 \mathrm{mg}, 0.45 \mathrm{mmol}, 1.5$ equiv.) in DMSO (3 mL) for $25 \mathrm{~min}$. The crude product was purified by column chromatography on $\mathrm{SiO}_{2}$ (pentane/EtOAc $=19: 1$ to 9:1) to afford $4 \mathbf{y}(138 \mathrm{mg}, 0.24 \mathrm{mmol}, 80 \%)$ with d.r. $>20: 1$.

Appearance: white solid.

TLC: $R_{f}=0.53$ (pentane/EtOAc $=8: 2$, UV-active and stains blue in vanillin).

${ }^{1} \mathrm{H}-\mathrm{NMR}\left(400 \mathrm{MHz}, \mathrm{CDCl}_{3}\right) \delta(\mathrm{ppm})=7.39-7.29(\mathrm{~m}, 5 \mathrm{H}), 5.19(\mathrm{t}, J=3.6 \mathrm{~Hz}, 1 \mathrm{H}), 5.09(\mathrm{~d}, J=$ $0.9 \mathrm{~Hz}, 2 \mathrm{H}), 3.22(\mathrm{~d}, J=9.2 \mathrm{~Hz}, 1 \mathrm{H}), 2.33-2.18(\mathrm{~m}, 2 \mathrm{H}), 2.00-1.79(\mathrm{~m}, 5 \mathrm{H}), 1.71(\mathrm{t}, J=13.6$ $\mathrm{Hz}, 1 \mathrm{H}), 1.67-1.53(\mathrm{~m}, 4 \mathrm{H}), 1.56-1.52(\mathrm{~m}, 2 \mathrm{H}), 1.52-1.33(\mathrm{~m}, 2 \mathrm{H}), 1.33-1.22(\mathrm{~m}, 6 \mathrm{H}), 1.14$ $(\mathrm{s}, 3 \mathrm{H}), 1.13-1.08(\mathrm{~m}, 1 \mathrm{H}), 1.07-1.01(\mathrm{~m}, 1 \mathrm{H}), 1.00(\mathrm{~s}, 3 \mathrm{H}), 0.97-0.88(\mathrm{~m}, 9 \mathrm{H}), 0.87(\mathrm{~s}, 3 \mathrm{H})$, $0.85(\mathrm{~s}, 3 \mathrm{H}), 0.80(\mathrm{~s}, 3 \mathrm{H}), 0.73(\mathrm{dd}, \mathrm{J}=11.5,1.9 \mathrm{~Hz}, 1 \mathrm{H})$.

${ }^{13} \mathrm{C}-\mathrm{NMR}\left(101 \mathrm{MHz}, \mathrm{CDCl}_{3}\right) \delta(\mathrm{ppm})=174.3,144.3,136.1,128.5,128.2,128.1,122.5,79.0$, $66.1,55.2,47.7,47.0,46.6$, 41.6, 39.8, 38.8, 38.6, 36.9, 34.8, 34.4, 33.2, 32.8, 32.5, 30.9, 28.4, $28.1,27.3,26.1,25.7,23.6,23.0,22.3,18.3,16.7,15.6,15.5,14.0$.

All the data are in accordance with the literature. ${ }^{[5]}$

Synthesis of derivative of 18B-Glycyrrhetinic acid (4z)
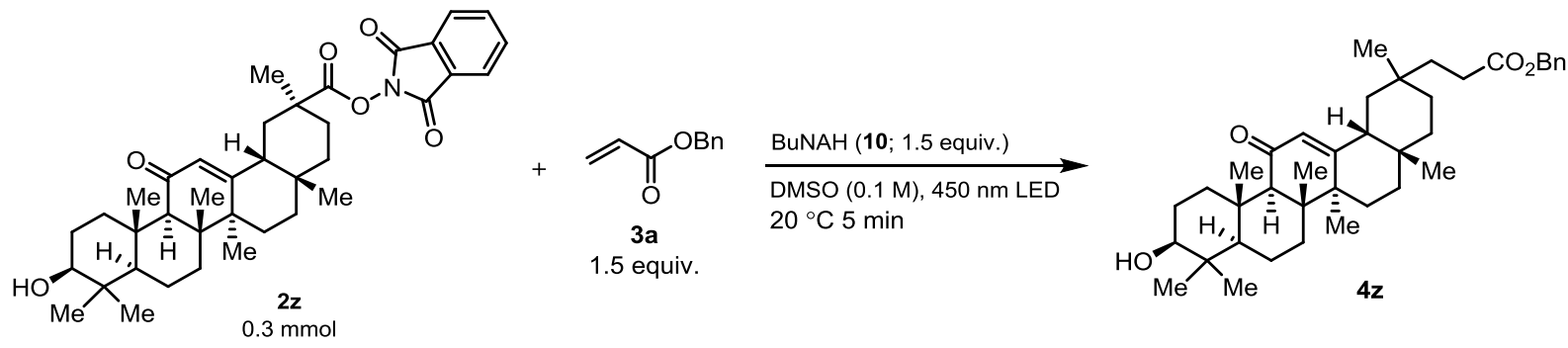

General procedure B3 was applied using redox-active ester of $18 \beta$-Glycyrrhetinic acid (2z; $184.7 \mathrm{mg}, 0.30 \mathrm{mmol}, 1.0$ equiv.), benzyl acrylate (3a; $73 \mathrm{mg}, 0.45 \mathrm{mmol}, 1.5$ equiv.), 1-butyl1,4-dihydropyridine-3-carboxamide (10; $81 \mathrm{mg}, 0.45 \mathrm{mmol}, 1.5$ equiv.) in DMSO (3 mL) for 5 min. The crude product was purified by column chromatography on $\mathrm{SiO}_{2}$ (pentane/EtOAc $=$ 9:1 to $8: 2$ ) to afford $\mathbf{4 z}(140 \mathrm{mg}, 0.23 \mathrm{mmol}, 79 \%)$ with d.r. $=1.3: 1$.

Appearance: white foam.

TLC: $R_{f}=0.68$ (pentane/EtOAc $=7: 3$, UV-active and stains blue in vanillin).

${ }^{1} \mathrm{H}-\mathrm{NMR}\left(400 \mathrm{MHz} \mathrm{CDCl}_{3}\right.$; mixture of diastereomers) $\delta(\mathrm{ppm})=7.39-7.29(\mathrm{~m}, 5 \mathrm{H}), 5.57(\mathrm{~s}$, $1 \mathrm{H}), 5.11(\mathrm{dd}, J=8.0,1.3 \mathrm{~Hz}, 2 \mathrm{H}), 3.25-3.19(\mathrm{~m}, 1 \mathrm{H}), 2.79(\mathrm{dq}, J=13.5,3.4 \mathrm{~Hz}, 1 \mathrm{H}), 2.37-$ $2.29(\mathrm{~m}, 2 \mathrm{H}), 2.24(\mathrm{t}, J=8.4 \mathrm{~Hz}, 1 \mathrm{H}), 2.15-1.96(\mathrm{~m}, 2 \mathrm{H}), 1.81(\mathrm{tt}, J=13.6,4.2 \mathrm{~Hz}, 1 \mathrm{H}), 1.71-$ $1.55(\mathrm{~m}, 7 \mathrm{H}), 1.49-1.39(\mathrm{~m}, 3 \mathrm{H}), 1.36-1.25(\mathrm{~m}, 6 \mathrm{H}), 1.22-1.04(\mathrm{~m}, 9 \mathrm{H}), 1.00(\mathrm{~s}, 3 \mathrm{H}), 0.99-$ $0.92(\mathrm{~m}, 2 \mathrm{H}), 0.85(\mathrm{~m}, 6 \mathrm{H}), 0.80(\mathrm{~m}, 3 \mathrm{H}), 0.73-0.65(\mathrm{~m}, 1 \mathrm{H})$.

${ }^{13} \mathrm{C}-\mathrm{NMR}$ (101 MHz, CDCl ; mixture of diastereomers) $\delta(\mathrm{ppm})=200.2,200.1,174.1,174.0$, $170.1,169.9,136.0,128.6,128.3,128.2,128.2$, 78.8, 77.2, 66.3, 66.3, 61.8, 55.0, 54.9, 47.1, 
$46.8,45.4,45.4,43.4,43.4,43.2,43.1,40.4,39.1,37.1,37.1,36.0,35.8,33.3,33.0,32.8,32.8$, $32.7,32.6,32.3,32.2,29.7,29.0,28.9,28.8,28.7,28.6,28.1,27.3,26.7,26.4,26.4,26.4,23.5$, 23.4, 20.5, 18.7, 17.5, 16.4, 15.6 .

HRMS (ESI-TOF) calc'd for $\mathrm{C}_{39} \mathrm{H}_{56} \mathrm{O}_{4} \mathrm{Na}[\mathrm{M}+\mathrm{Na}]^{+}: 611.4071$; found: 611.4085 .

Synthesis of derivative of 186-Glycyrrhetinic acid (4aa)
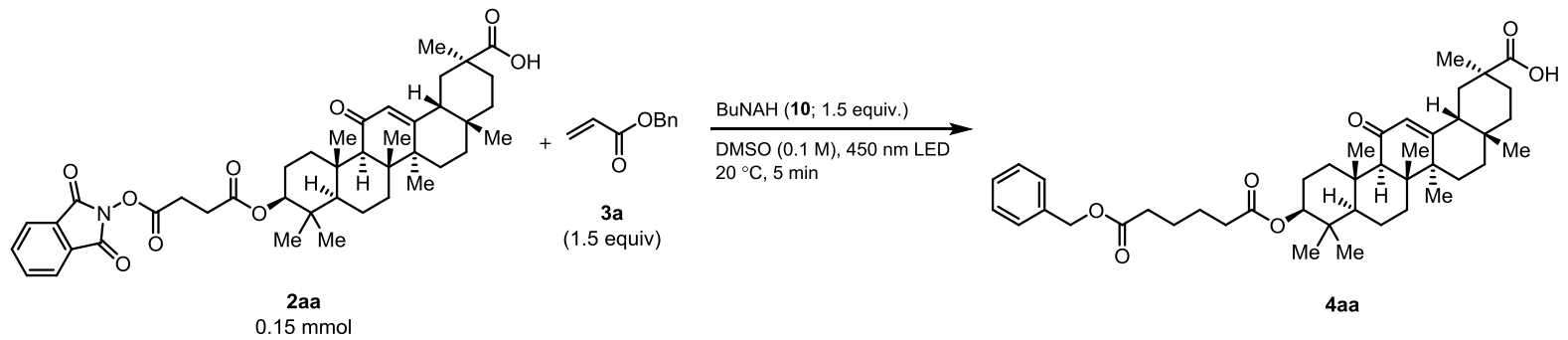

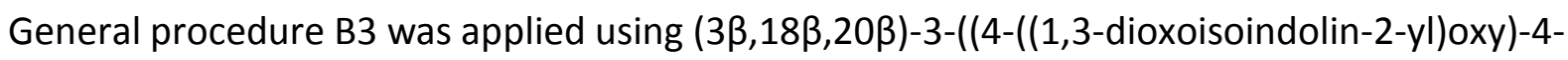
oxobutanoyl)oxy)-11-oxo-olean-12-en-29-oic acid (2aa; $110 \mathrm{mg}, 0.15 \mathrm{mmol}, 1.0$ equiv.), benzyl acrylate (3a; $37.4 \mathrm{mg}, 0.23 \mathrm{mmol}, 1.5$ equiv.), and 1-butyl-1,4-dihydropyridine-3carboxamide (10; $41.5 \mathrm{mg}, 0.23 \mathrm{mmol}, 1.5$ equiv.) in DMSO $(1.5 \mathrm{~mL})$ for $5 \mathrm{~min}$. The crude product was purified by preparative TLC $($ DCM/EtOAC $=1: 1)$ to afford 4aa $(48.0 \mathrm{mg}, 0.07$ $\mathrm{mmol}, 45 \%)$.

Appearance: white solid.

TLC: $R_{f}=0.34(D C M / M e O H=20: 1$, UV-active).

M.pt. $=229.5-230.6^{\circ} \mathrm{C}$.

${ }^{1} \mathrm{H}-\mathrm{NMR}\left(400 \mathrm{MHz}, \mathrm{CDCl}_{3}\right) \delta(\mathrm{ppm})=7.40-7.29(\mathrm{~m}, 5 \mathrm{H}), 5.71(\mathrm{~s}, 1 \mathrm{H}), 5.11(\mathrm{~s}, 2 \mathrm{H}), 4.52(\mathrm{dd}, J$ $=11.6,4.9 \mathrm{~Hz}, 1 \mathrm{H}), 2.79(\mathrm{dt}, J=13.6,3.7 \mathrm{~Hz}, 1 \mathrm{H}), 2.44-2.26(\mathrm{~m}, 5 \mathrm{H}), 2.23-2.13(\mathrm{~m}, 1 \mathrm{H}), 2.08$ $-1.91(\mathrm{~m}, 3 \mathrm{H}), 1.88-1.77(\mathrm{~m}, 1 \mathrm{H}), 1.75-1.52(\mathrm{~m}, 8 \mathrm{H}), 1.51-1.33(\mathrm{~m}, 8 \mathrm{H}), 1.31(\mathrm{~d}, J=6.1 \mathrm{~Hz}$, $1 \mathrm{H}), 1.28-1.19(\mathrm{~m}, 5 \mathrm{H}), 1.16(\mathrm{~s}, 3 \mathrm{H}), 1.13(\mathrm{~s}, 3 \mathrm{H}), 1.08-1.01(\mathrm{~m}, 2 \mathrm{H}), 0.86(\mathrm{~d}, J=2.9 \mathrm{~Hz}, 6 \mathrm{H})$, $0.84(\mathrm{~s}, 3 \mathrm{H}), 0.82-0.77(\mathrm{~m}, 1 \mathrm{H})$.

${ }^{13} \mathrm{C}-\mathrm{NMR}\left(101 \mathrm{MHz}, \mathrm{CDCl}_{3}\right) \delta(\mathrm{ppm})=200.5,181.6,173.3,173.2,169.6,136.1,128.7,128.6$, $128.4,128.3$, 80.7, 66.4, 61.8, 55.2, 48.4, 45.6, 43.9, 43.4, 41.0, 38.9, 38.2, 37.9, 37.1, 34.5, $34.1,32.8,32.0,31.1,28.7,28.6,28.2,26.6,26.6,24.7,24.6,23.7,23.5,18.8,17.5,16.9,16.5$. HRMS (ESI-TOF) calc'd for $\mathrm{C}_{43} \mathrm{H}_{60} \mathrm{NO}{ }_{7} \mathrm{Na}[\mathrm{M}+\mathrm{Na}]^{+}:$711.4231; found: 711.4222 . 


\section{Alkyl photo-coupling on DNA.}

\section{LCMS Analysis}

Liquid chromatography-mass spectrometry (HPLC-MS) analyses were performed on Agilent 1260 Infinity II system using an Electrospray ionization (ESI) ion source.

Column: Reverse phase (YMC-Triart C18, $5 \mu \mathrm{m}$ particle size, $4.6 \mathrm{~mm} \times 150 \mathrm{~mm}$ ); column temperature: $40{ }^{\circ} \mathrm{C}$; flow rate: $0.8 \mathrm{~mL} / \mathrm{min}$.

Mobile phase: (A). $0.038 \%$ v/v Et ${ }_{3} \mathrm{~N}, 0.75 \%$ v/v HFIP in $\mathrm{H}_{2} \mathrm{O}$; (B). $0.038 \%$ v/v Et $\mathrm{t}_{3} \mathrm{~N}, 0.75 \%$ v/v HFIP in 9:1 $\mathrm{MeOH} / \mathrm{H}_{2} \mathrm{O}$.

Gradient: 5 to $95 \%$ B over $10 \mathrm{~min}$.

UV detection: $260 \mathrm{~nm}$.

MS data: range: $500-2000 \mathrm{~m} / \mathrm{z}$; ionization polarity: negative, triply charged mass was observed $(\mathrm{M}-3) / 3$.

Sample preparation: $10-20 \mu \mathrm{L}$ of DNA stock solution $(0.1 \mathrm{mM})$ in a mixture of $0.038 \% \mathrm{v} / \mathrm{v} \mathrm{Et} 3 \mathrm{~N}, 0.75 \%$ v/v HFIP in $\mathrm{H}_{2} \mathrm{O} / \mathrm{MeOH}(95.5: 4.5)(130$ $\mu \mathrm{L})$

\section{Injection amount: $20 \mu \mathrm{L}$.}

Synthesis of DNA acrylate $\mathbf{1 2 a}$
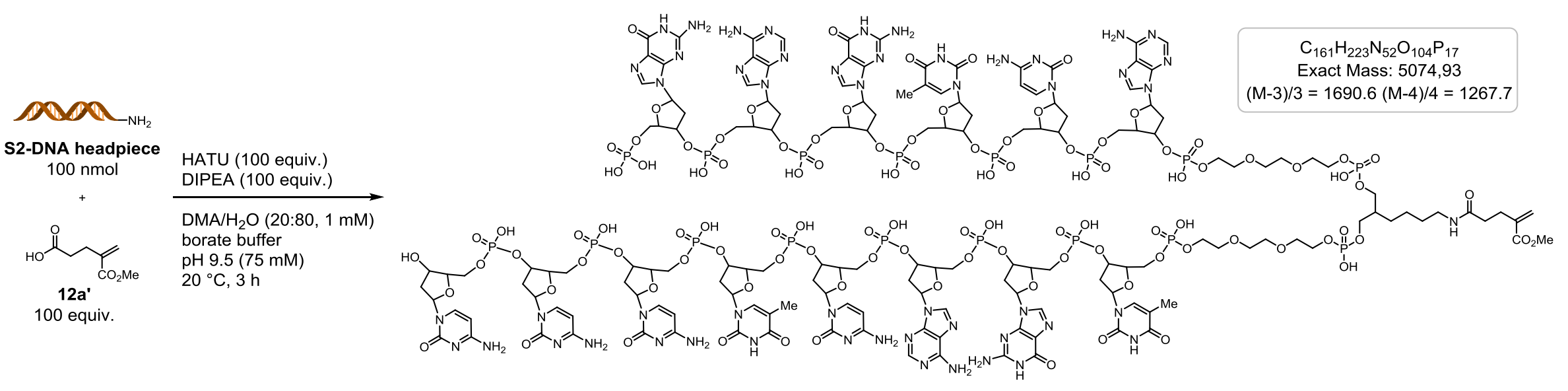

12 
The DNA acrylate 12a was synthesized according to the literature procedure. ${ }^{[17]}$ A 1.0 M solution of 4-(methoxycarbonyl)pent-4-enoic acid (12a'; $10 \mu \mathrm{L}, 10 \mu \mathrm{mol}, 100$ equiv.) in $\mathrm{H}_{2} \mathrm{O}$ and $\mathrm{N}, \mathrm{N}$-diisopropylethylamine (DIPEA; $10 \mu \mathrm{L}, 10 \mu \mathrm{mol}, 1.0 \mathrm{M}$ in DMA, 100 equiv.) were added in an Eppendorf tube containing 1-[bis(dimethylamino)methylene]-1H-1,2,3-triazolo[4,5-b]pyridinium 3-oxide hexafluorophosphate (HATU; $3.8 \mathrm{mg}, 10 \mu \mathrm{mol}, 100$ equiv.). The reaction mixture was vortexed and allowed to stand at room temperature for $1 \mathrm{~h}$, followed by the addition to a solution of the DNA headpiece (S2; $5 \mu \mathrm{L}, 20 \mathrm{mM}$ in $\mathrm{H}_{2} \mathrm{O}, 0.1 \mu \mathrm{mol}, 1.0$ equiv.) in borate buffer ( $\left.75 \mu \mathrm{L}, 100 \mathrm{mM}, \mathrm{pH} 9.5\right)$. The mixture was vortexed and allowed to stand at room temperature for $2 \mathrm{~h}$. Sodium chloride $\left(10 \mu \mathrm{L}, 5.0 \mathrm{M}\right.$ in $\left.\mathrm{H}_{2} \mathrm{O}\right)$ and cold ethanol $(0.3 \mathrm{~mL})$ were added. The resulting mixture was vortexed and allowed to stand at $-20^{\circ} \mathrm{C}$ for $30 \mathrm{~min}$. The suspension was centrifuged at $12000 \mathrm{RPM}$ for $3 \mathrm{~min}$. After discarding the supernatant, the pellet was dried under reduced pressure and redissolved in $\mathrm{H}_{2} \mathrm{O}(200 \mu \mathrm{L})$ to afford a stock solution of DNA acrylate $12 \mathrm{a}(0.5 \mathrm{mM})$.
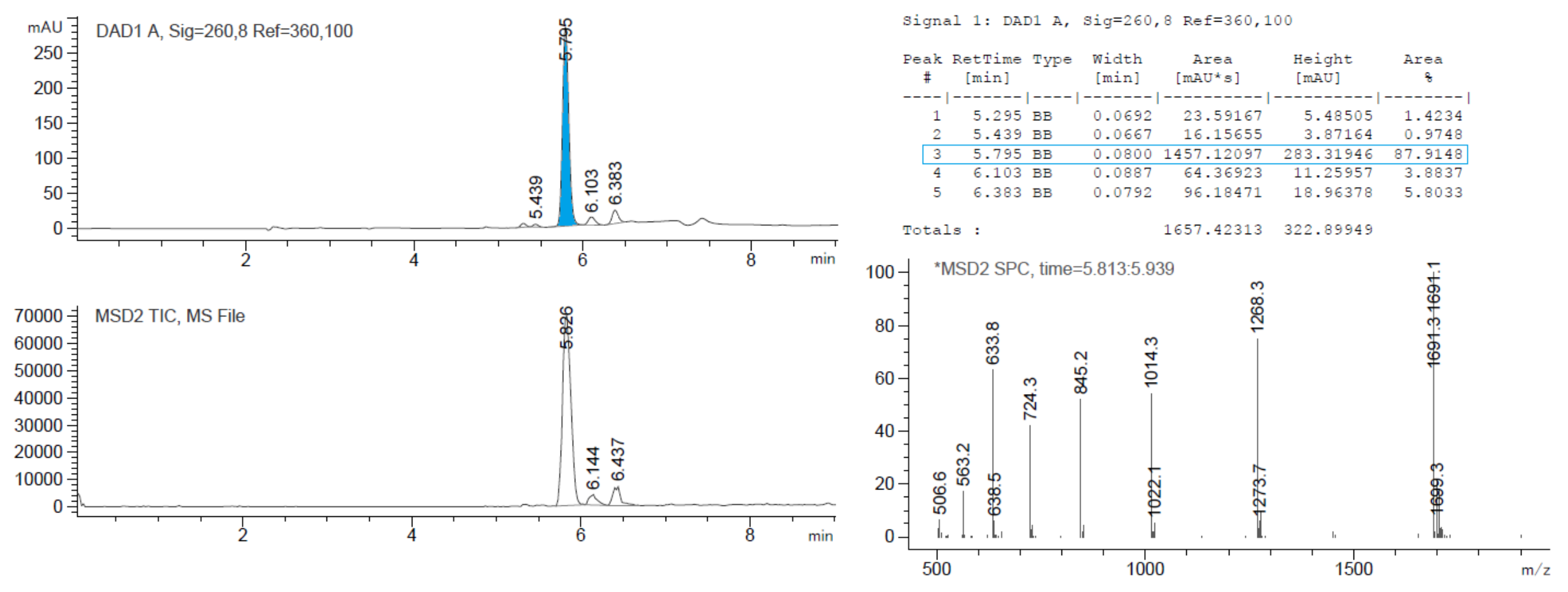

Figure S4: LCMS traces of DNA acrylate $12 a$.

The by-products formed in the synthesis of DNA acrylates were not taken into account for the yield determination of the following photo-ligated coupling reactions. 

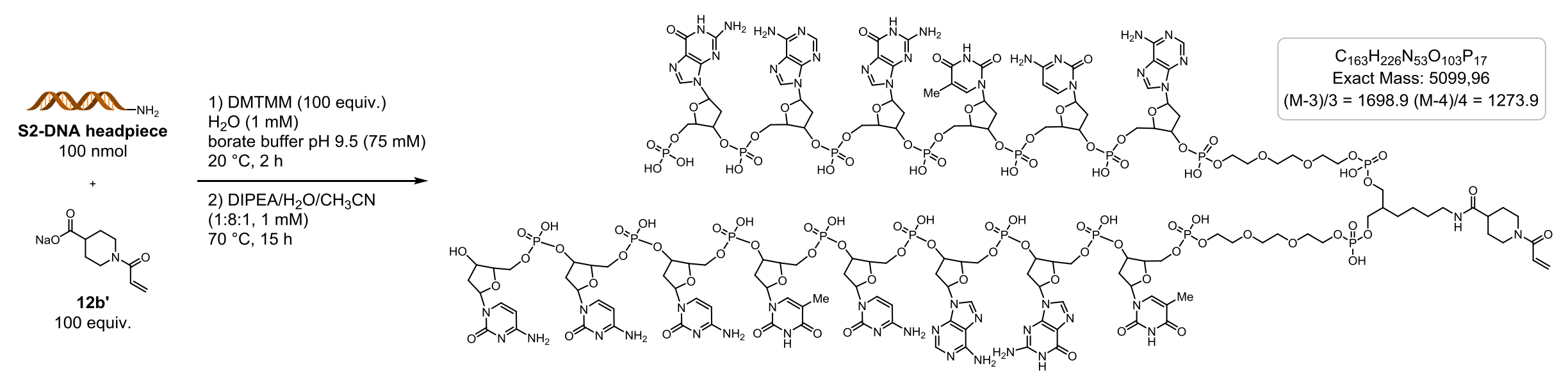

12b

The DNA amide $\mathbf{1 2} \mathbf{b}$ was synthesized according to the literature procedure. ${ }^{[17]}$ Sodium 1-acryloylpiperidine-4-carboxylate (12b') was prepared as a $1.0 \mathrm{M}$ solution in $\mathrm{H}_{2} \mathrm{O}$ using 1-acryloylpiperidine-4-carboxylic acid ( $1.8 \mathrm{mg}, 10 \mu \mathrm{mol}, 100$ equiv.) and sodium hydroxide ( $0.40 \mathrm{mg}, 10 \mu \mathrm{mol}, 100$ equiv.). Sodium 1-acryloylpiperidine-4-carboxylate (12b', $10 \mu \mathrm{L}, 10 \mu \mathrm{mol}, 1.0 \mathrm{M}$ in $\mathrm{H}_{2} \mathrm{O}, 100$ equiv.), 4-(4,6-dimethoxy-1,3,5-triazin-2-yl)-4methylmorpholinium chloride (DMTMM; $10 \mu \mathrm{L}, 10 \mu \mathrm{mol}, 1.0 \mathrm{M}$ in $\mathrm{H}_{2} \mathrm{O}, 100$ equiv.), borate buffer (75 $\left.\mu \mathrm{L}, 100 \mathrm{mM}, \mathrm{pH} 9.5\right)$, and DNA headpiece (S2; $5 \mu \mathrm{L}, 20 \mathrm{mM}$ in $\mathrm{H}_{2} \mathrm{O}, 0.1 \mu \mathrm{mol}, 1.0$ equiv.) were added in a $1.5 \mathrm{~mL}$ Eppendorf tube. The reaction mixture was vortexed and allowed to stand at room temperature for $2 \mathrm{~h}$. Sodium chloride $\left(10 \mu \mathrm{L}, 5.0 \mathrm{M}\right.$ in $\left.\mathrm{H}_{2} \mathrm{O}\right)$ and cold ethanol $(0.3 \mathrm{~mL})$ were added to the mixture. The suspension was mixed by vortex and allowed to stand at $-20^{\circ} \mathrm{C}$ for $30 \mathrm{~min}$. Then, the mixture was centrifuged at $12000 \mathrm{RPM}$ for 3 min and the supernatant was discarded. The pellet obtained was dried under reduced pressure and dissolved in a mixture of $\mathrm{H}_{2} \mathrm{O}(80 \mu \mathrm{L}), \mathrm{MeCN}(10 \mu \mathrm{L}), \mathrm{and} N, N$ diisopropylethylamine (DIPEA ; $10 \mu \mathrm{L}$ ). The resulting mixture was heated at $70{ }^{\circ} \mathrm{C}$ for $15 \mathrm{~h}$. After cooling to room temperature, sodium chloride $\left(10 \mu \mathrm{L}, 5.0 \mathrm{M} \mathrm{in} \mathrm{H}_{2} \mathrm{O}\right)$ and cold ethanol $(0.3 \mathrm{~mL})$ were added. The mixture was vortexed and allowed to stand at $-20^{\circ} \mathrm{C}$ for $30 \mathrm{~min}$. The suspension was centrifuged at 12000 RPM for 3 min and after discarding the supernatant, the pellet was dried under reduced pressure and redissolved in $\mathrm{H}_{2} \mathrm{O}(200 \mu \mathrm{L})$ to afford a stock solution of DNA acrylamide $12 \mathbf{b}(0.5 \mathrm{mM})$. 


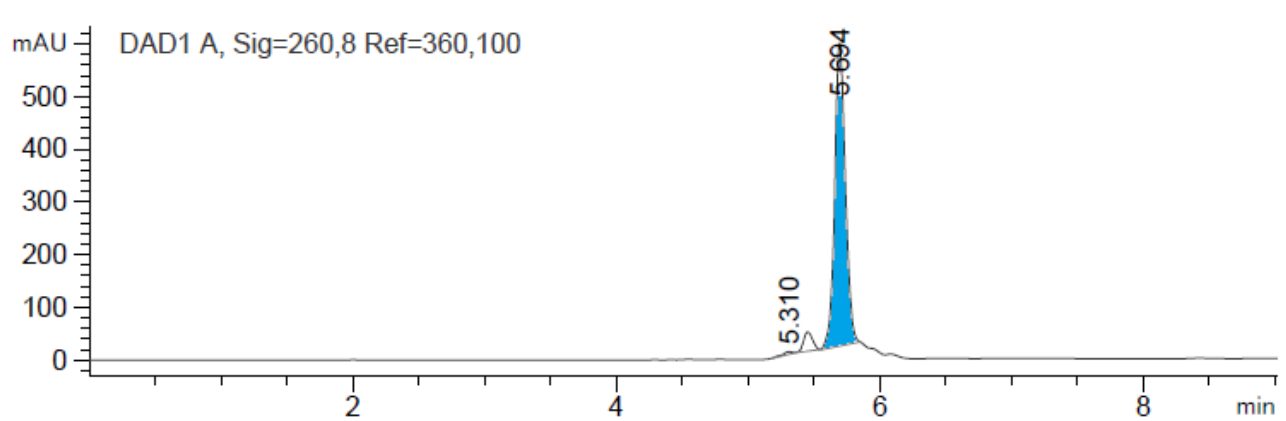

Signal 1: DAD1 A, Sig $=260,8$ Ref $=360,100$

\begin{tabular}{|c|c|c|c|c|c|c|}
\hline $\begin{array}{c}\text { Peak } \\
\#\end{array}$ & $\begin{array}{c}\text { RetTime } \\
{[\mathrm{min}]}\end{array}$ & Type & $\begin{array}{l}\text { Width } \\
\text { [min] }\end{array}$ & $\begin{array}{c}\text { Area } \\
{\left[\mathrm{MAU}^{*} \mathrm{~s}\right]}\end{array}$ & $\begin{array}{l}\text { Height } \\
{[\mathrm{MAU}]}\end{array}$ & $\begin{array}{c}\text { Area } \\
\&\end{array}$ \\
\hline 1 & 5.310 & $\mathrm{BB}$ & 0.0818 & 27.02697 & 4.86410 & 0.7942 \\
\hline 2 & 5.453 & BB & 0.0728 & 169.27588 & 36.69874 & 4.9744 \\
\hline 3 & 5.694 & MM & 0.0928 & 3206.64648 & 575.70593 & 94.2314 \\
\hline
\end{tabular}
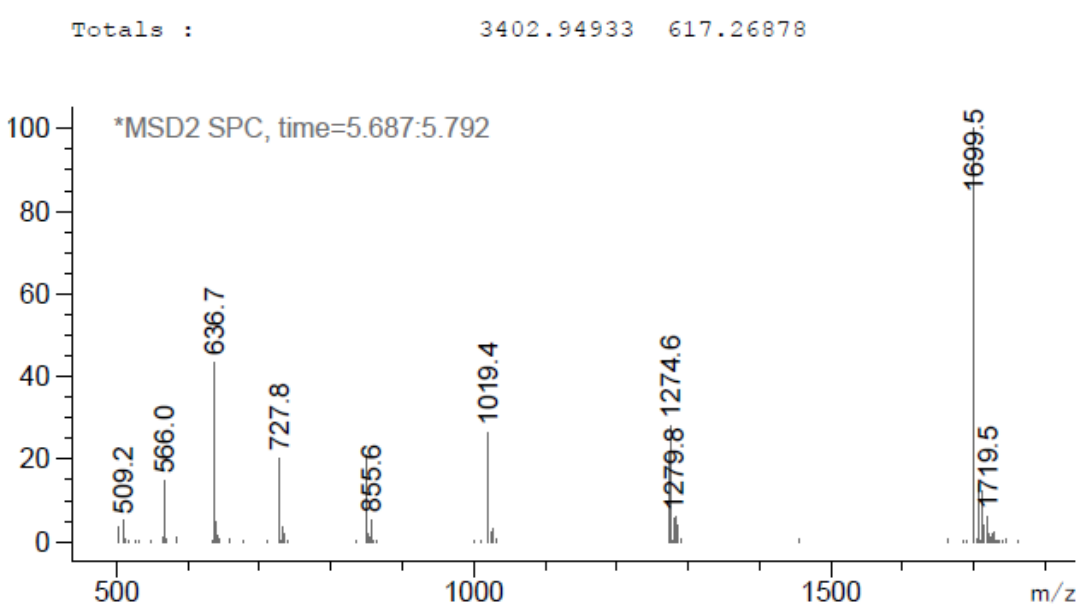

Figure S5: LCMS traces of DNA acrylamide $\mathbf{1 2 b}$. 
General procedure (C): Photo-mediated decarboxylative coupling of redox-active esters with DNA Michael acceptors.

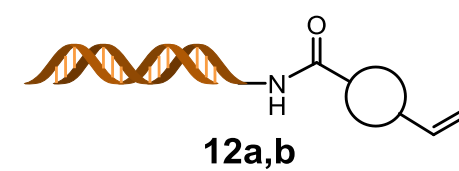

$12 \mathbf{a}, \mathbf{b}$
$10 \mathrm{nmol}$

$+$

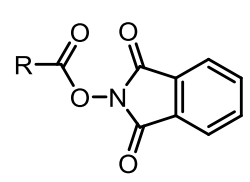

2

30 equiv.

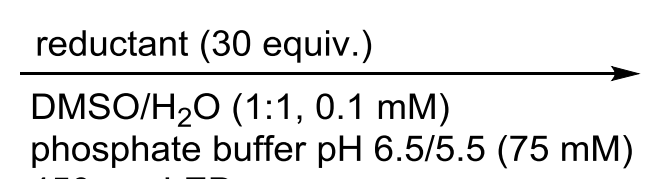

phosphate buffer pH 6.5/5.5 (75 mM)

$450 \mathrm{~nm}$ LED

$20^{\circ} \mathrm{C}, 1$ to $4 \mathrm{~h}$

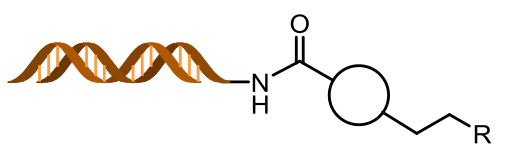

13aa-ae,ba-be

DNA containing electron poor olefin 12a,b (20 $\mu \mathrm{L}, 0.5 \mathrm{mM}$ in $\mathrm{H}_{2} \mathrm{O}, 10 \mathrm{nmol}, 1$ equiv.), redox-active ester 2 ( $3 \mathrm{mM}$ in DMSO, $300 \mathrm{nmol}, 30$ equiv.), BuNAH (10; 3 mM in DMSO, $300 \mathrm{nmol}, 30$ equiv.) or NADH (11; $3 \mathrm{mM}$ in DMSO, $300 \mathrm{nmol}, 30$ equiv.), DMSO (30 $\mu \mathrm{L}$ ), and phosphate buffer (30 $\mu \mathrm{L} 250 \mathrm{mM}, \mathrm{pH}$ 6.5) were added to a screw top vial. The vial was closed with a septum, evacuated for $2 \mathrm{~s}$ and refilled with argon (six times). The mixture was then irradiated with blue LEDs for 1 to $4 \mathrm{~h}$ at room temperature (using a fan from the top for cooling the reaction mixture). Sodium chloride $\left(10 \mu \mathrm{L}, 5.0 \mathrm{M}\right.$ in $\left.\mathrm{H}_{2} \mathrm{O}\right)$ and cold ethanol $(0.3 \mathrm{~mL})$ were added. The mixture was vortexed and allowed to stand at $-20^{\circ} \mathrm{C}$ for $30 \mathrm{~min}$. The suspension was centrifuged at 12000 RPM for $3 \mathrm{~min}$, and after discarding the supernatant, the pellet was dried under reduced pressure and redissolved in $\mathrm{H}_{2} \mathrm{O}(100 \mu \mathrm{L})$ to give a stock solution of DNA product 13aa-ae,ba-be $(0.1 \mathrm{mM})$.

\section{Yield determination of the photo mediated decarboxylative coupling reaction of DNA acrylates}

The yield of the photo-mediated decarboxylative coupling reaction of DNA acrylates was determined by LC-MS as reported in the literature for DEL synthesis ${ }^{[17-20]}$ by integration of the UV absorbance at $260 \mathrm{~nm}$, assuming total DNA recovery and that all DNA compounds have similar UV absorbance. To calculate the yield the area of the product was divided by the summed up area of all DNA containing compounds. Non-DNA impurities (molecular weight $<1000 \mathrm{~g} / \mathrm{mol}$ ) were not included in the yield calculation.

$$
\text { Yield }(\%)=\frac{\text { Area }(\text { peak of the DNA product })}{\text { Area }(\text { all DNA containing compounds })}
$$




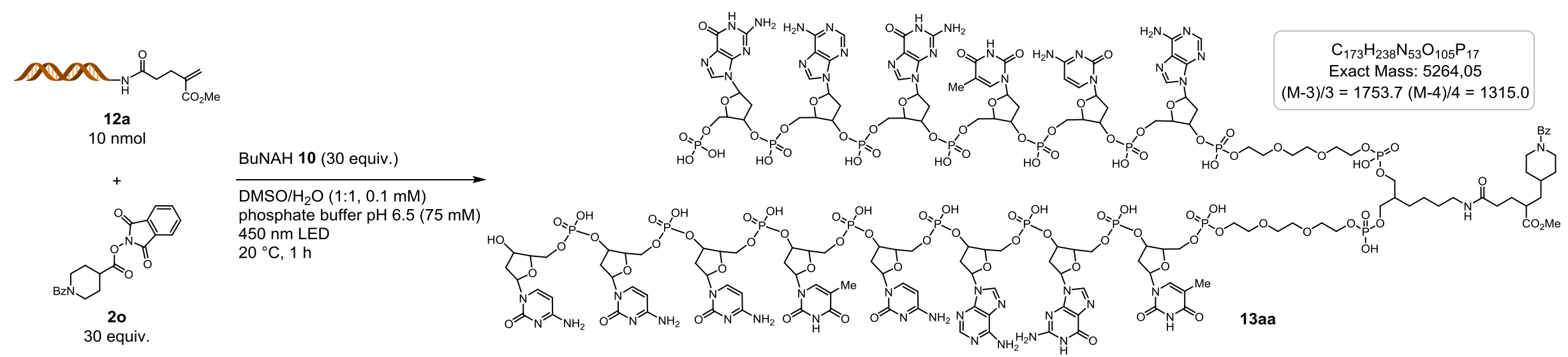

General procedure $\mathrm{C}$ was applied using DNA acrylate $12 \mathrm{a}\left(20 \mu \mathrm{L}, 0.5 \mathrm{mM}\right.$ in $\mathrm{H}_{2} \mathrm{O}, 10 \mathrm{nmol}, 1.0$ equiv.), redox-active ester 20 (10 $\mu \mathrm{L}, 30 \mathrm{mM}$ in DMSO, $0.30 \mu \mathrm{mol}, 30$ equiv.), BuNAH (10; $10 \mu \mathrm{L}, 30 \mathrm{mM}$ in DMSO, $0.30 \mu \mathrm{mol}, 30$ equiv.), and phosphate buffer (30 $\mu \mathrm{L}, 250 \mathrm{mM}, \mathrm{pH} 6.5)$ in DMSO $(30 \mu \mathrm{L})$ for $1 \mathrm{~h}$. Yield: $78 \%$ (4\% s.m.)
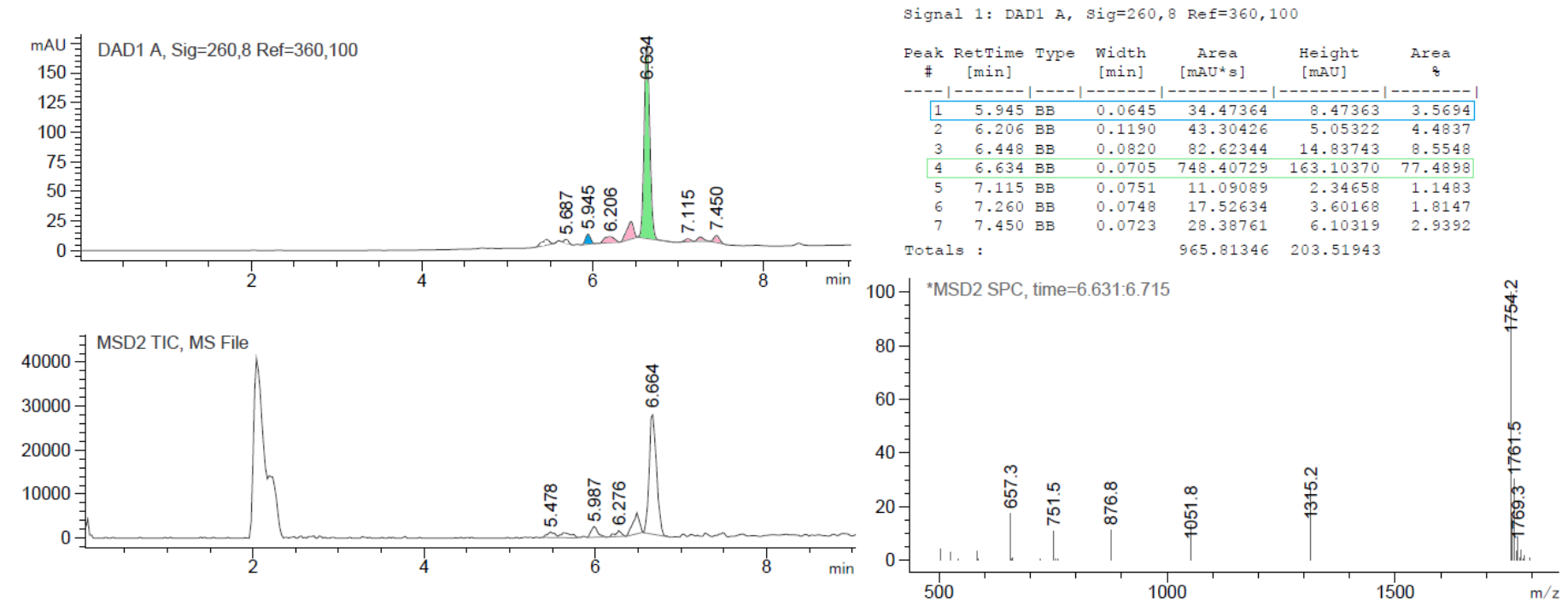

Figure S6: LCMS trace of BUNAH (10) promoted DNA coupled product 13aa (green) 


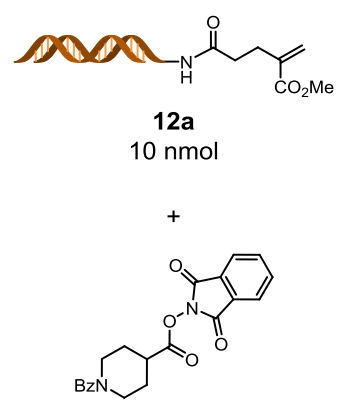

20

\section{NADH 11 (30 equiv.)}

$\mathrm{DMSO} / \mathrm{H}_{2} \mathrm{O}(1: 1,0.1 \mathrm{mM})$

phosphate buffer pH $6.5(75 \mathrm{mM})$ $40^{\circ} \mathrm{C}, 2 \mathrm{~h}$ ${ }^{\circ} \mathrm{C}, 2 \mathrm{~h}$

General procedure $C$ was applied using DNA acrylate $12 \mathrm{a}\left(20 \mu \mathrm{L}, 0.5 \mathrm{mM}\right.$ in $\mathrm{H}_{2} \mathrm{O}, 10 \mathrm{nmol}, 1.0$ equiv.), redox-active ester 20 (10 $\mu \mathrm{L}, 30 \mathrm{mM}$ in DMSO, $0.30 \mu \mathrm{mol}, 30$ equiv.), NADH (11; $10 \mu \mathrm{L}, 30 \mathrm{mM}$ in DMSO, $0.30 \mu \mathrm{mol}, 30$ equiv.), and phosphate buffer (30 $\mu \mathrm{L}, 250 \mathrm{mM}, \mathrm{pH} 6.5)$ in DMSO (30 $\mu \mathrm{L}$ ) for $2 \mathrm{~h}$. Yield: $88 \%$ (2\% s.m.)
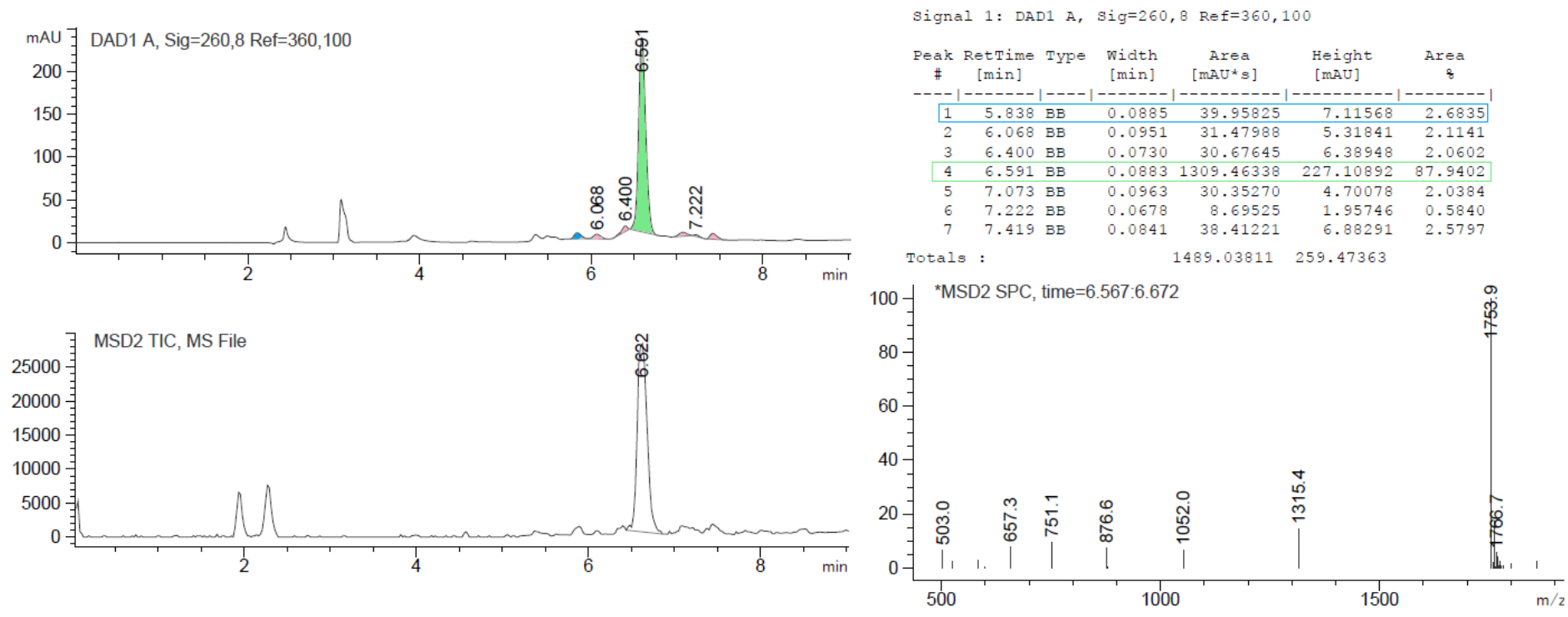

Figure S7: LCMS trace of NADH (11) promoted DNA coupled product 13aa (green) 


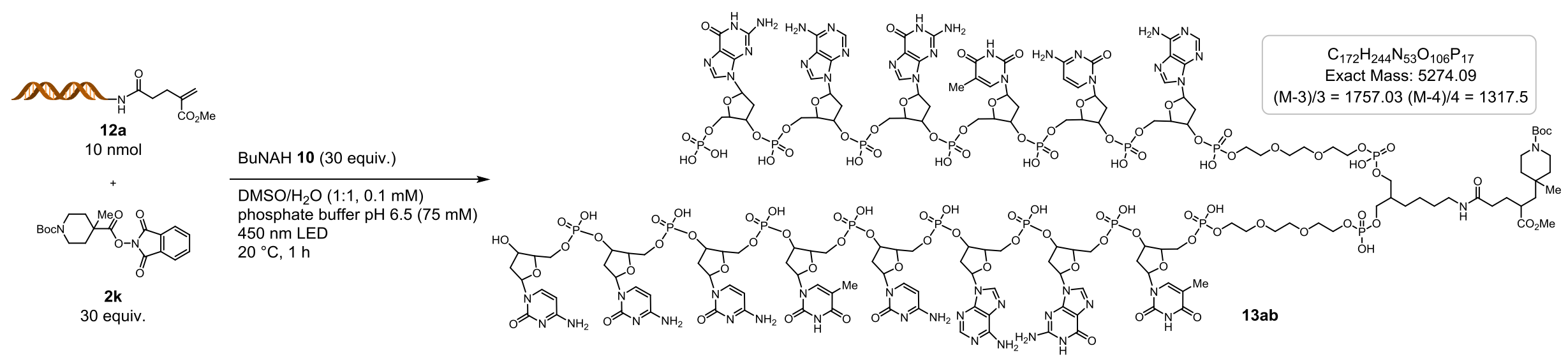

General procedure $C$ was applied using DNA acrylate $12 \mathrm{a}\left(20 \mu \mathrm{L}, 0.5 \mathrm{mM}\right.$ in $\mathrm{H}_{2} \mathrm{O}, 10 \mathrm{nmol}, 1.0$ equiv.), redox-active ester $\mathbf{2 k}(10 \mu \mathrm{L}, 30 \mathrm{mM}$ in DMSO, $0.30 \mu \mathrm{mol}, 30$ equiv.), BuNAH (10; $10 \mu \mathrm{L}, 30 \mathrm{mM}$ in DMSO, $0.30 \mu \mathrm{mol}, 30$ equiv.), and phosphate buffer (30 $\mu \mathrm{L}, 250 \mathrm{mM}, \mathrm{pH} 6.5)$ in DMSO $(30 \mu \mathrm{L})$ for $1 \mathrm{~h}$. Yield: $81 \%$ (0\% s.m.)
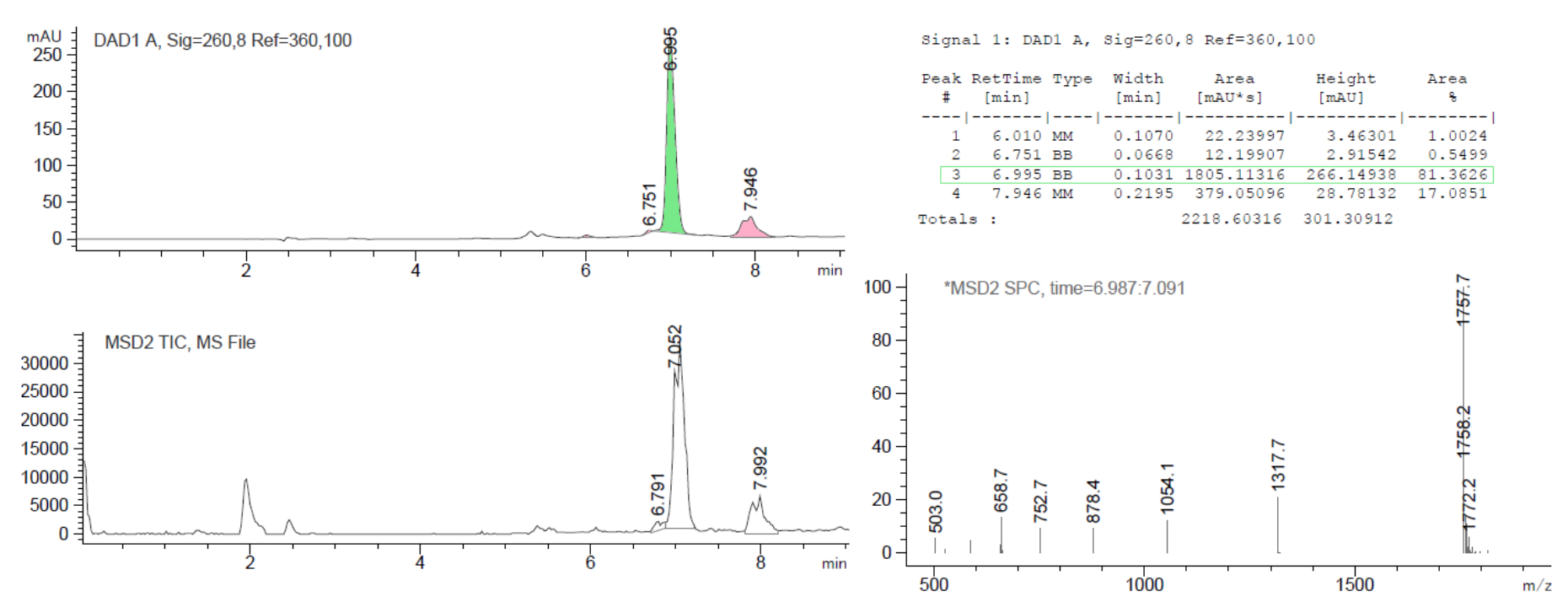

Figure S8: LCMS trace of BUNAH (10) promoted DNA coupled product 13ab (green) 


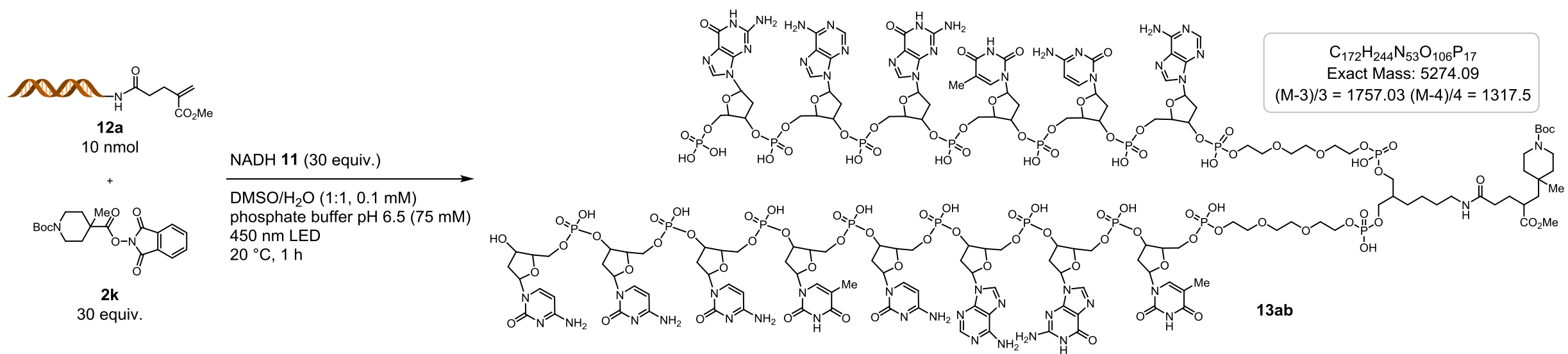

General procedure $C$ was applied using DNA acrylate $12 \mathrm{a}\left(20 \mu \mathrm{L}, 0.5 \mathrm{mM}\right.$ in $\mathrm{H}_{2} \mathrm{O}, 10 \mathrm{nmol}, 1.0$ equiv.), redox-active ester $\mathbf{2 k}(10 \mu \mathrm{L}, 30 \mathrm{mM}$ in DMSO, $0.30 \mu \mathrm{mol}, 30$ equiv.), NADH (11; $10 \mu \mathrm{L}, 30 \mathrm{mM}$ in DMSO, $0.30 \mu \mathrm{mol}, 30$ equiv.), and phosphate buffer (30 $\mu \mathrm{L}, 250 \mathrm{mM}, \mathrm{pH} 6.5)$ in DMSO (30 $\mu$ L) for $1 \mathrm{~h}$. Yield: $77 \%$ (0\% s.m.)
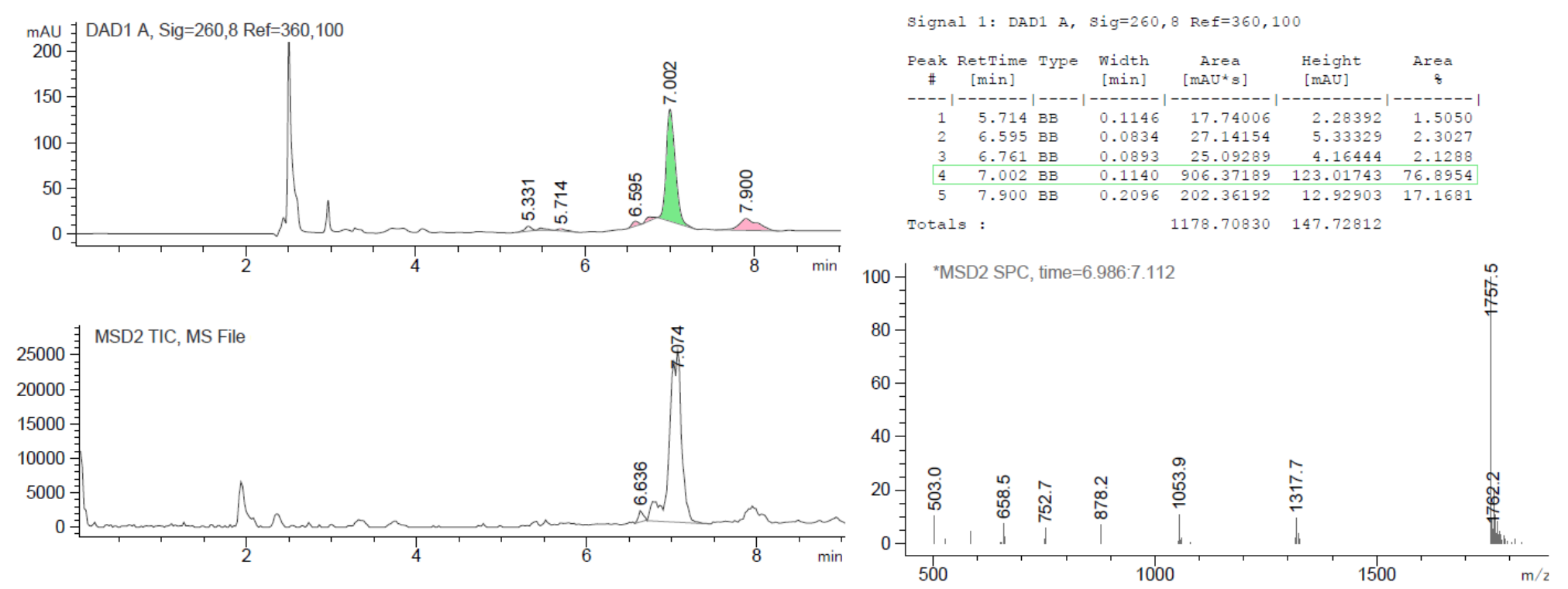

Figure S9: LCMS trace of NADH (11) promoted DNA coupled product 13ab (green) 
Synthesis of DNA functionalized product 13ac using BuNAH (10) as a reductant

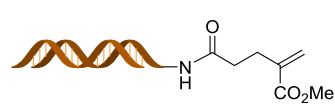

$12 \mathrm{a}$
$10 \mathrm{nmol}$

\section{BUNAH 10 (30 equiv.)}

DMSO/ $\mathrm{H}_{2} \mathrm{O}(1: 1,0.1 \mathrm{mM})$

phosphate buffer pH $6.5(75 \mathrm{mM})$

$20^{\circ} \mathrm{C}, 1 \mathrm{~h}$
$40 \mathrm{~nm}$

2a

30 equiv.
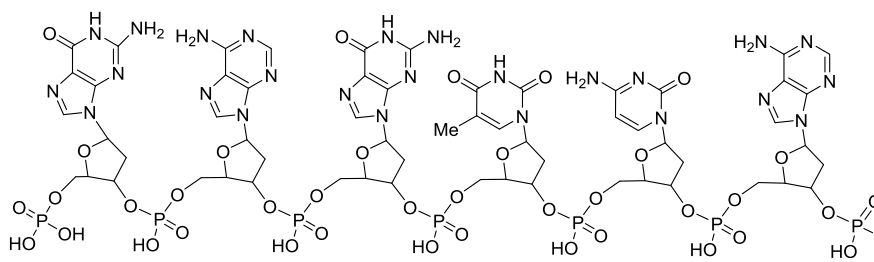

$\mathrm{C}_{168} \mathrm{H}_{237} \mathrm{~N}_{52} \mathrm{O}_{104} \mathrm{P}_{17}$

Exact Mass: 5173.04

$(M-3) / 3=1723.3(M-4) / 4=1292.3$

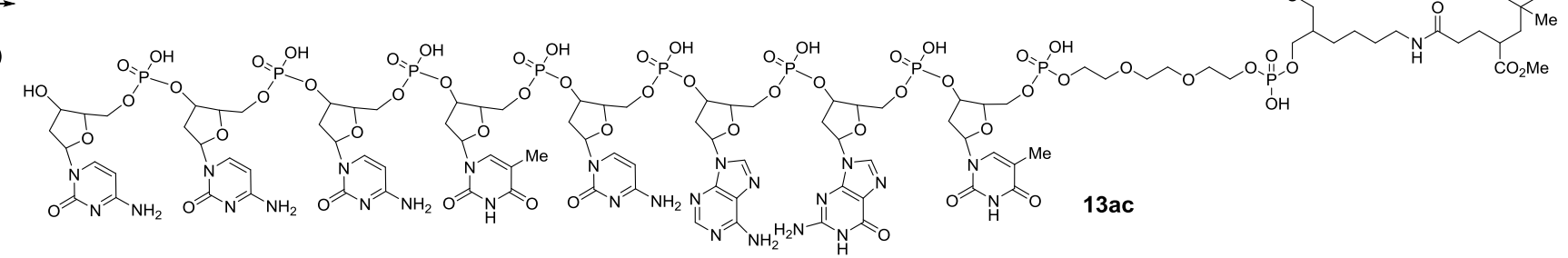

General procedure $\mathrm{C}$ was applied using DNA acrylate $12 \mathrm{a}\left(20 \mu \mathrm{L}, 0.5 \mathrm{mM}\right.$ in $\mathrm{H}_{2} \mathrm{O}, 10 \mathrm{nmol}, 1.0$ equiv.), redox-active ester $2 \mathrm{a}(10 \mu \mathrm{L}, 30 \mathrm{mM}$ in DMSO, $0.30 \mu \mathrm{mol}, 30$ equiv.), BuNAH (10; $10 \mu \mathrm{L}, 30 \mathrm{mM}$ in DMSO, $0.30 \mu \mathrm{mol}, 30$ equiv.) and phosphate buffer (30 $\mu \mathrm{L}, 250 \mathrm{mM}, \mathrm{pH} 6.5)$ in DMSO (30 $\mu$ L), for 1 h. Yield: 91\% (0\% s.m.)
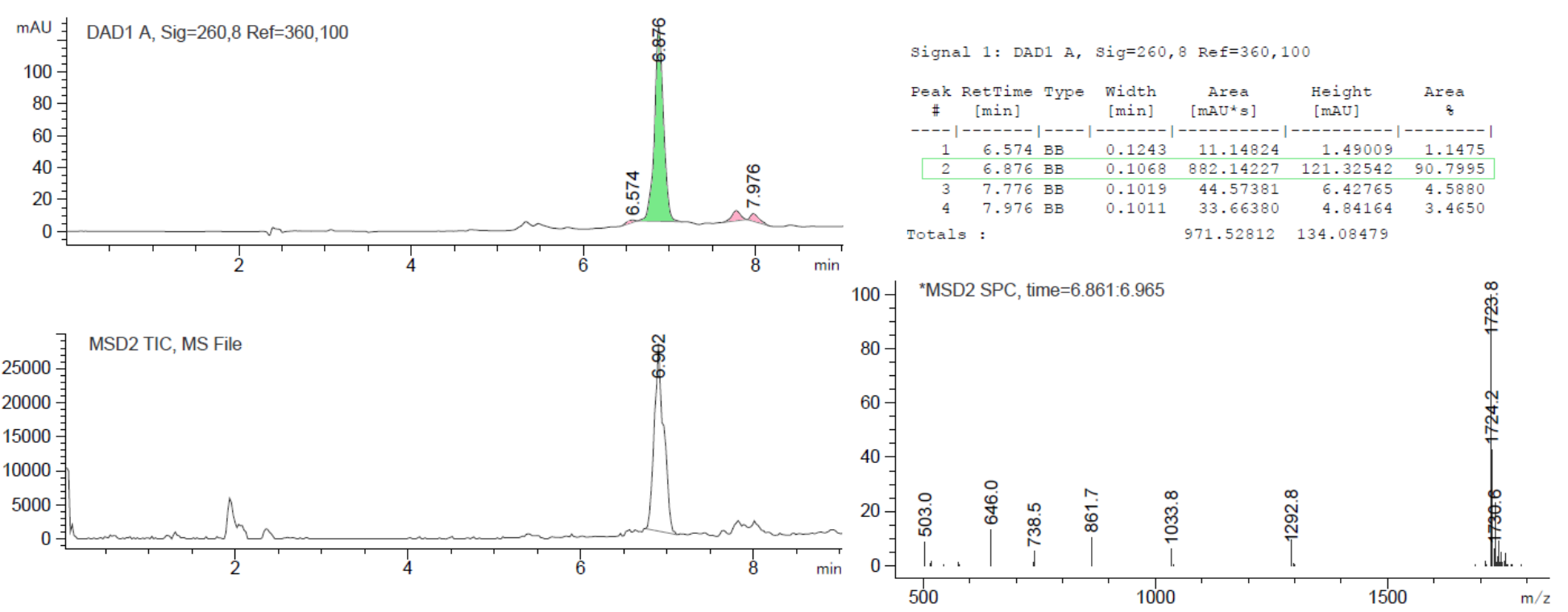

Figure S10: LCMS trace of BUNAH (10) promoted DNA coupled product 13ac (green) 
Synthesis of DNA functionalized product 13ac using NADH (11) as a reductant

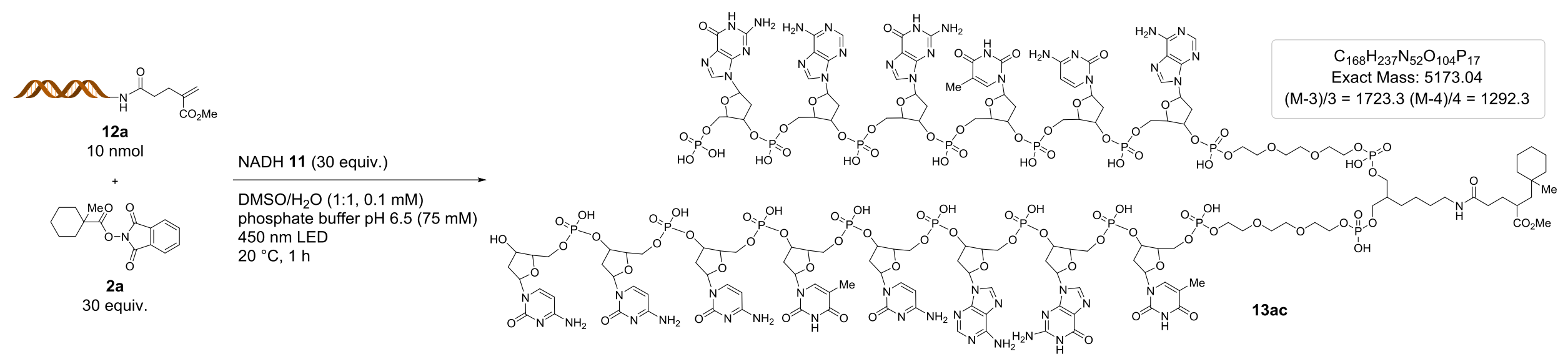

General procedure $C$ was applied using DNA acrylate $12 \mathrm{a}\left(20 \mu \mathrm{L}, 0.5 \mathrm{mM}\right.$ in $\mathrm{H}_{2} \mathrm{O}, 10 \mathrm{nmol}, 1.0$ equiv.), redox-active ester $2 \mathrm{a}$ (10 $\mu \mathrm{L}, 30 \mathrm{mM}$ in DMSO, $0.30 \mu \mathrm{mol}, 30$ equiv.), NADH (11; $10 \mu \mathrm{L}, 30 \mathrm{mM}$ in DMSO, $0.30 \mu \mathrm{mol}, 30$ equiv.), and phosphate buffer (30 $\mu \mathrm{L}, 250 \mathrm{mM}, \mathrm{pH} 6.5)$ in DMSO (30 $\mu \mathrm{L}$ ) for $1 \mathrm{~h}$. Yield: $83 \%$ (0\% s.m.)
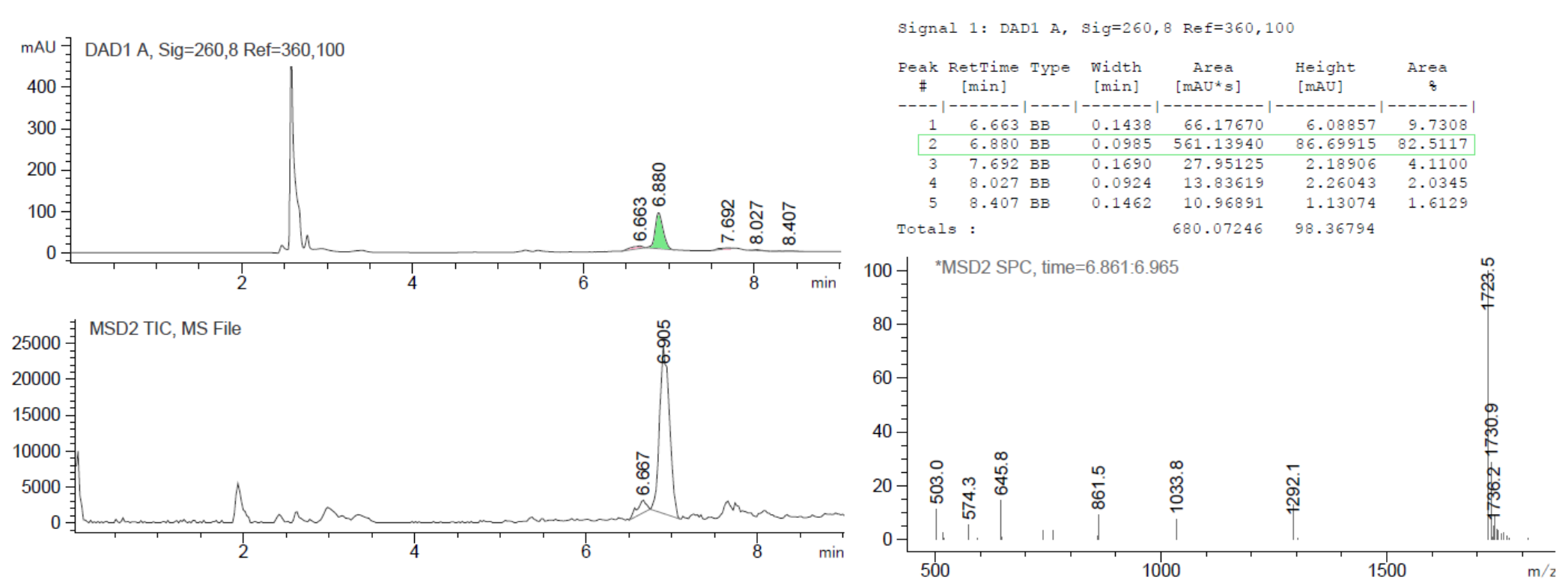

Figure S11: LCMS trace of NADH (11) promoted DNA coupled product 13ac (green) 
Synthesis of DNA functionalized product 13ad using BuNAH (10) as a reductant

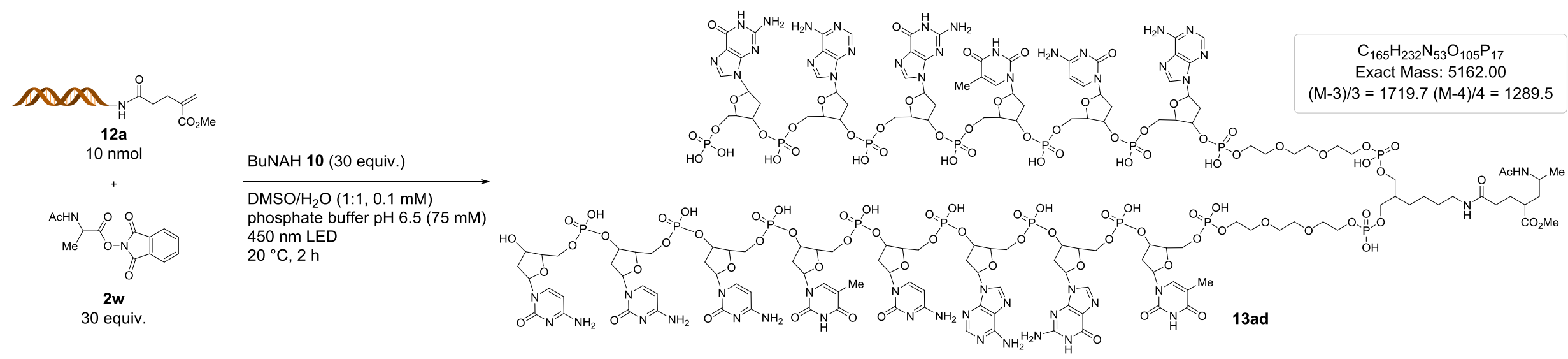

General procedure $C$ was applied using DNA acrylate $12 \mathrm{a}\left(20 \mu \mathrm{L}, 0.5 \mathrm{mM}\right.$ in $\mathrm{H}_{2} \mathrm{O}, 10 \mathrm{nmol}, 1.0$ equiv.), redox-active ester $2 \mathbf{w}$ (10 $\mu \mathrm{L}, 30 \mathrm{mM}$ in DMSO, $0.30 \mu \mathrm{mol}, 30$ equiv.), BuNAH (10; $10 \mu \mathrm{L}, 30 \mathrm{mM}$ in DMSO, $0.30 \mu \mathrm{mol}, 30$ equiv.), and phosphate buffer (30 $\mu \mathrm{L}, 250 \mathrm{mM}, \mathrm{pH} 6.5)$ in DMSO $(30 \mu \mathrm{L})$ for 2 h. Yield: $92 \%$ (0\% s.m.)
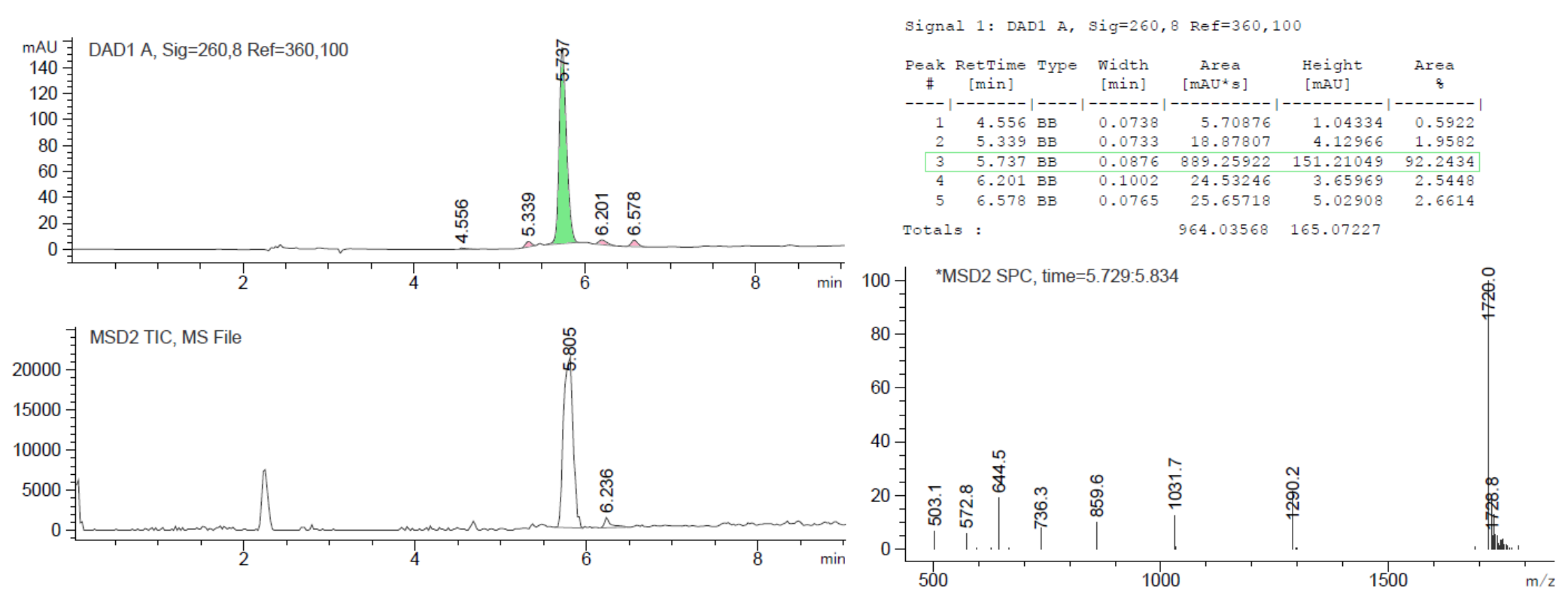

Figure S12: LCMS trace of BUNAH (10) promoted DNA coupled product 13ad (green) 


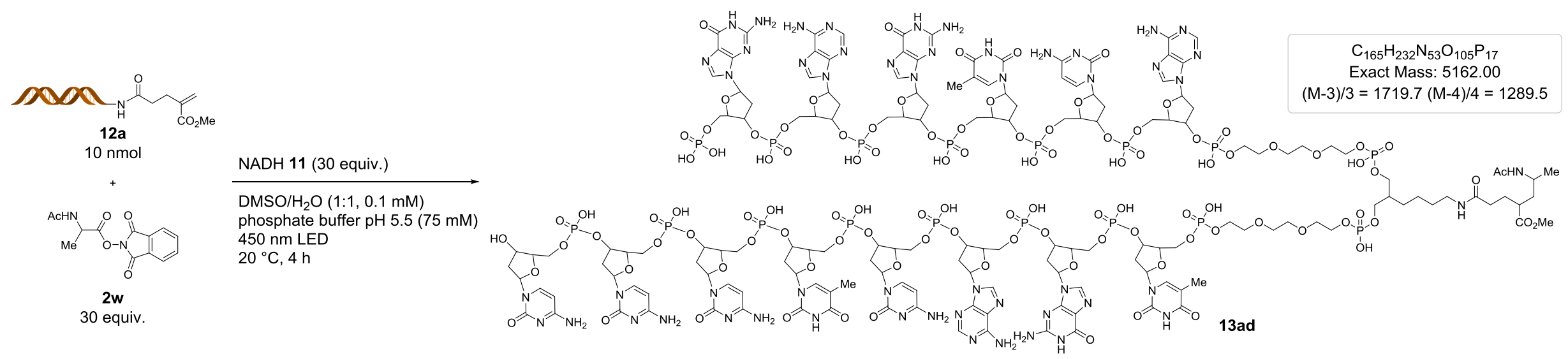

General procedure $C$ was applied using DNA acrylate $12 \mathrm{a}\left(20 \mu \mathrm{L}, 0.5 \mathrm{mM}\right.$ in $\mathrm{H}_{2} \mathrm{O}, 10 \mathrm{nmol}, 1.0$ equiv.), redox-active ester $2 \mathbf{w}$ (10 $\mu \mathrm{L}, 30 \mathrm{mM}$ in DMSO, $0.30 \mu \mathrm{mol}, 30$ equiv.), NADH (11; $10 \mu \mathrm{L}, 30 \mathrm{mM}$ in DMSO, $0.30 \mu \mathrm{mol}, 30$ equiv.), and phosphate buffer (30 $\mu \mathrm{L}, 250 \mathrm{mM}, \mathrm{pH} 6.5)$ in DMSO $(30 \mu \mathrm{L})$ for 4 h. Yield: $92 \%$ (0\% s.m.)
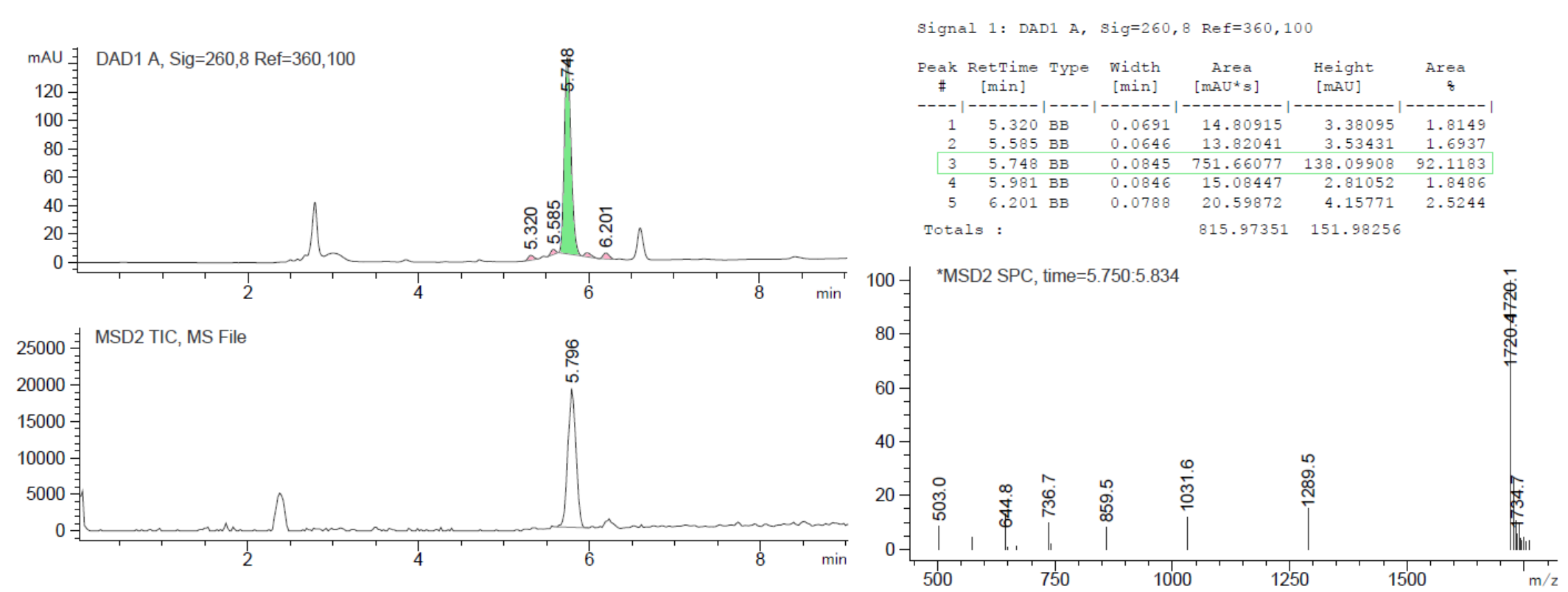

Figure S13: LCMS trace of NADH (11) promoted DNA coupled product 13ad (green) 


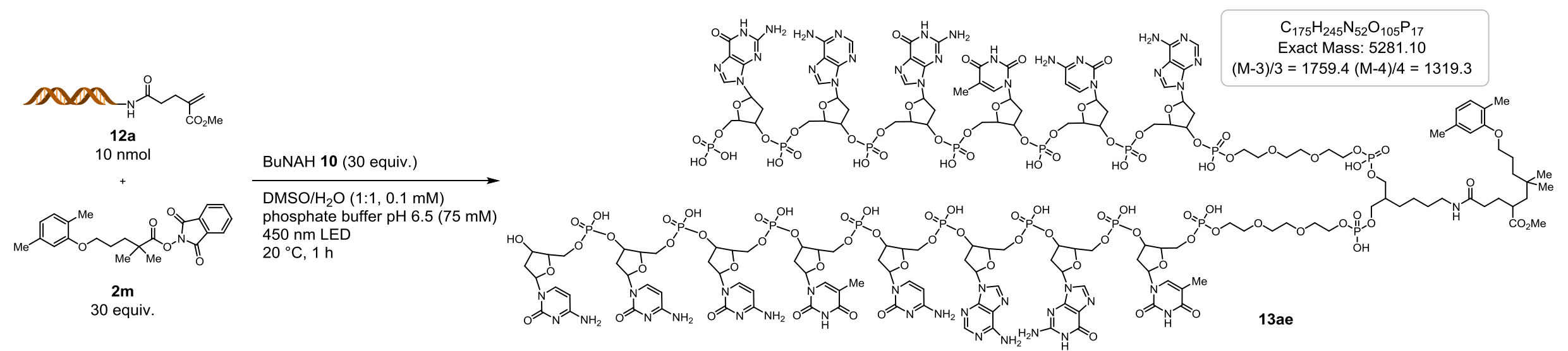

General procedure $C$ was applied using DNA acrylate $12 \mathrm{a}\left(20 \mu \mathrm{L}, 0.5 \mathrm{mM}\right.$ in $\mathrm{H}_{2} \mathrm{O}, 10 \mathrm{nmol}, 1.0$ equiv.), redox-active ester $2 \mathrm{~m}(10 \mu \mathrm{L}, 30 \mathrm{mM}$ in DMSO, $0.30 \mu \mathrm{mol}, 30$ equiv.), BuNAH (10; $10 \mu \mathrm{L}, 30 \mathrm{mM}$ in DMSO, $0.30 \mu \mathrm{mol}, 30$ equiv.), and phosphate buffer (30 $\mu \mathrm{L}, 250 \mathrm{mM}, \mathrm{pH} 6.5)$ in DMSO $(30 \mu \mathrm{L})$ for $1 \mathrm{~h}$. Yield: $86 \%$ (14\% s.m.)
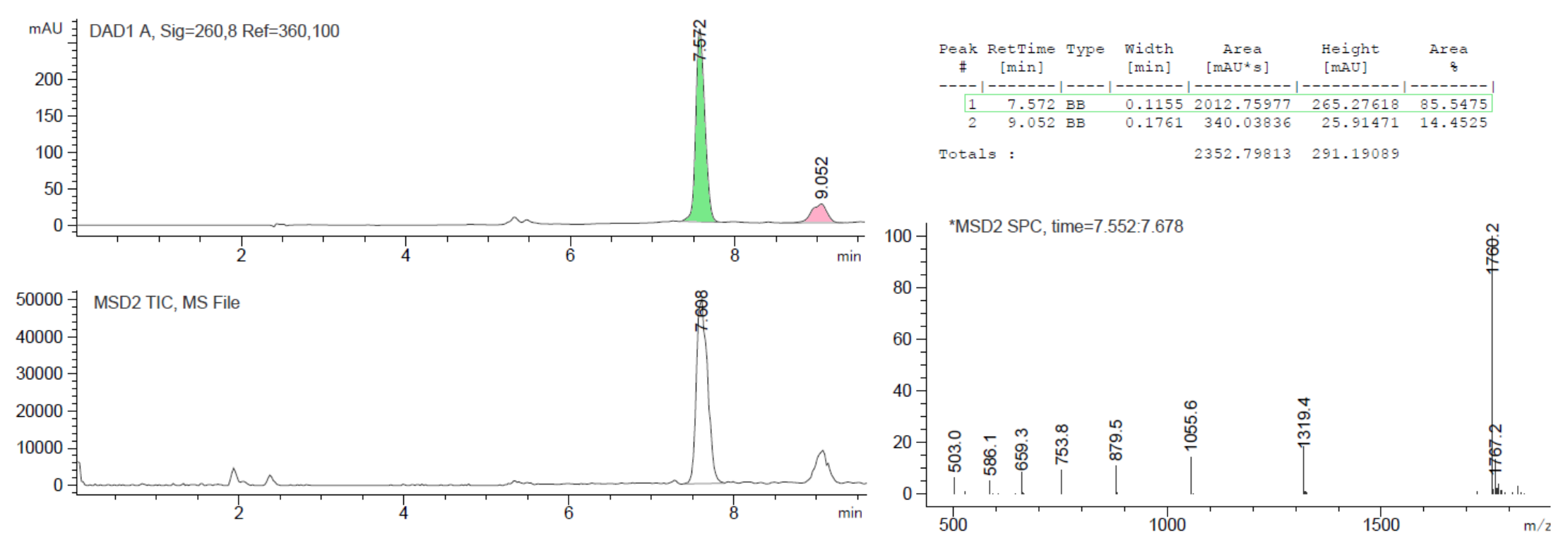

Figure S14: LCMS trace of BUNAH (10) promoted DNA coupled product 13ae (green) 
Synthesis of DNA functionalized product 13ad using NADH (11) as a reductant

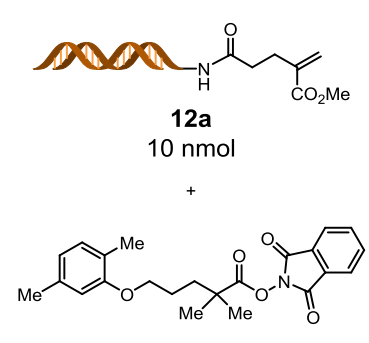

$2 m$
NADH 11 (30 equiv.)

$\mathrm{DMSO} / \mathrm{H}_{2} \mathrm{O}(1: 1,0.1 \mathrm{mM})$

phosphate buffer $\mathrm{pH} 6.5(75 \mathrm{mM})$

$450 \mathrm{~nm}$ LED
$20^{\circ} \mathrm{C}, 1 \mathrm{~h}$
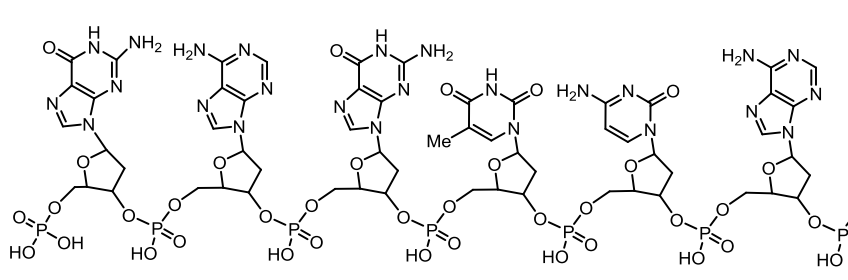

$\mathrm{C}_{175} \mathrm{H}_{245} \mathrm{~N}_{52} \mathrm{O}_{105} \mathrm{P}_{17}$

Exact Mass: 5281.10

$(\mathrm{M}-3) / 3=1759.4(\mathrm{M}-4) / 4=1319.3$

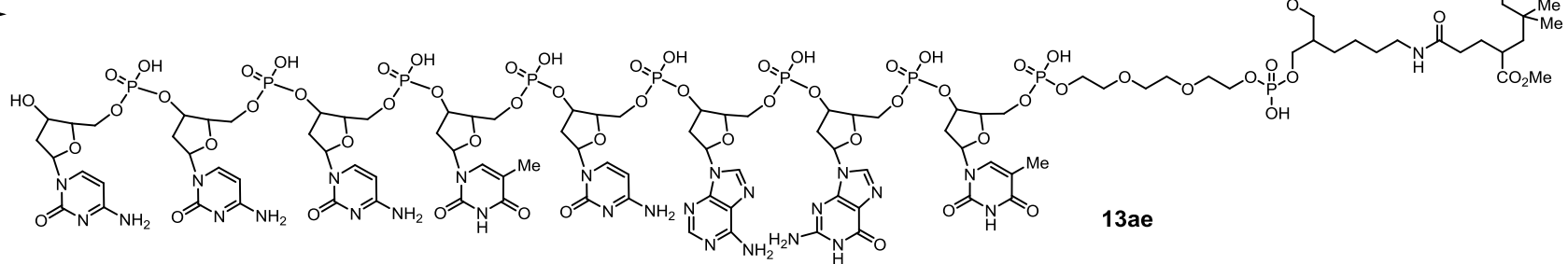

General procedure $C$ was applied using DNA acrylate $12 \mathrm{a}\left(20 \mu \mathrm{L}, 0.5 \mathrm{mM}\right.$ in $\mathrm{H}_{2} \mathrm{O}, 10 \mathrm{nmol}, 1.0$ equiv.), redox-active ester $2 \mathrm{~m}$ (10 $\mu \mathrm{L}, 30 \mathrm{mM}$ in DMSO, $0.30 \mu \mathrm{mol}, 30$ equiv.), NADH (11; $10 \mu \mathrm{L}, 30 \mathrm{mM}$ in DMSO, $0.30 \mu \mathrm{mol}, 30$ equiv.), and phosphate buffer (30 $\mu \mathrm{L}, 250 \mathrm{mM}, \mathrm{pH} 6.5)$ in DMSO $(30 \mu \mathrm{L}$ ) for $1 \mathrm{~h}$. Yield: $59 \%$ (4\% s.m.)
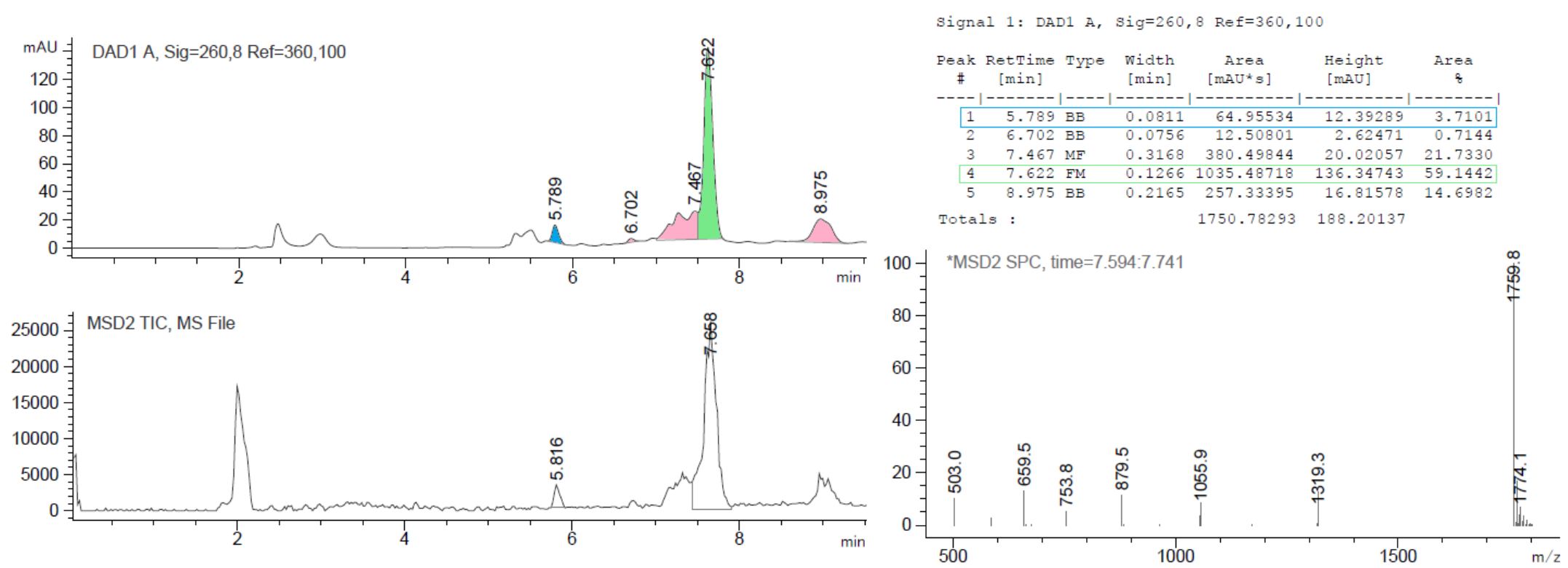

Figure S15: LCMS trace of NADH (11) promoted DNA coupled product 13ae (green) 


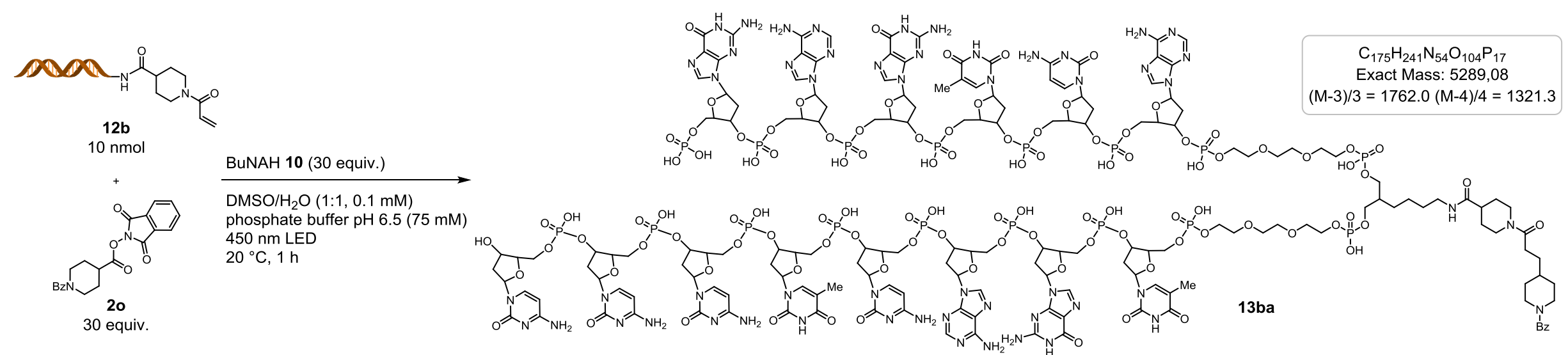

General procedure $C$ was applied using DNA amide $\mathbf{1 2 b}\left(20 \mu \mathrm{L}, 0.5 \mathrm{mM}\right.$ in $\mathrm{H}_{2} \mathrm{O}, 10 \mathrm{nmol}, 1.0$ equiv.), redox-active ester 20 (10 $\mu \mathrm{L}$, $30 \mathrm{mM}$ in DMSO, $0.30 \mu \mathrm{mol}, 30$ equiv.), BuNAH (10; $10 \mu \mathrm{L}, 30 \mathrm{mM}$ in DMSO, $0.30 \mu \mathrm{mol}, 30$ equiv.), and phosphate buffer (30 $\mu \mathrm{L}, 250 \mathrm{mM}, \mathrm{pH} 6.5)$ in DMSO $(30 \mu \mathrm{L})$ for $1 \mathrm{~h}$. Yield: $83 \%$ (10\% s.m.)
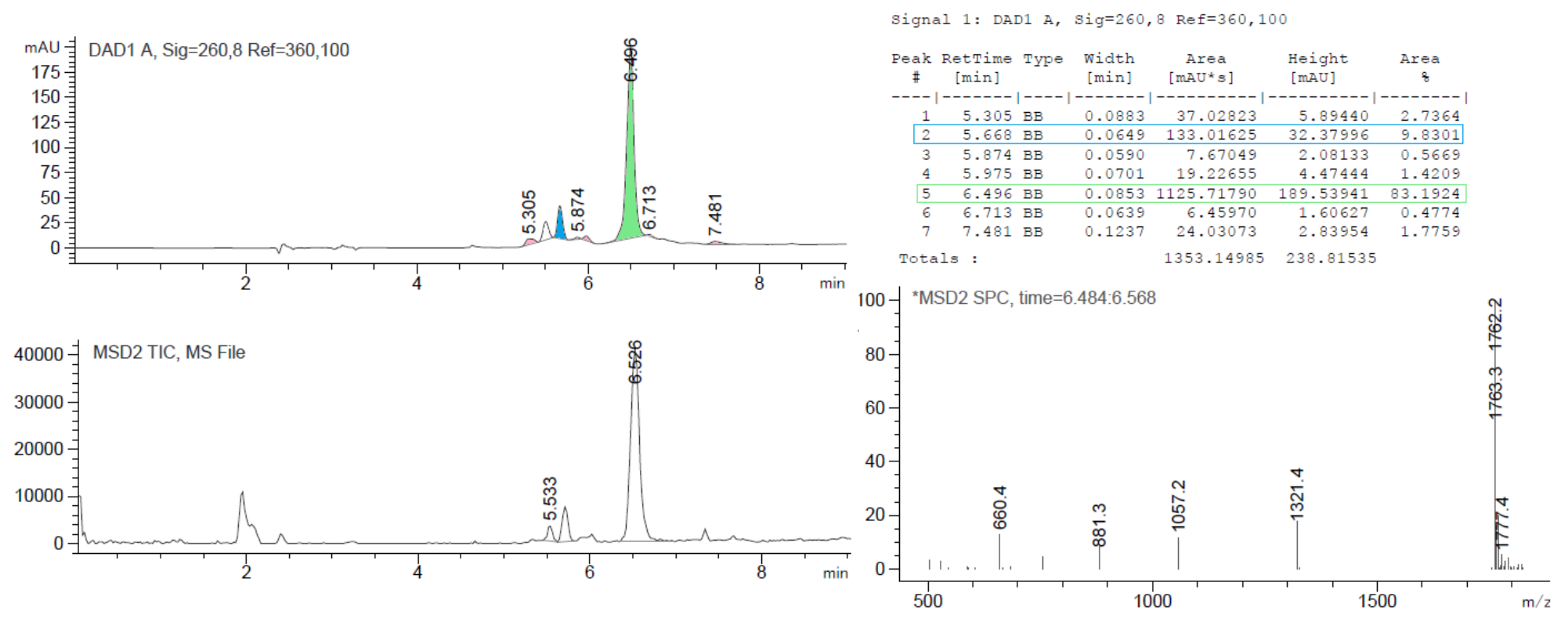

Figure S16: LCMS trace of BUNAH (10) promoted DNA coupled product 13ba (green) 


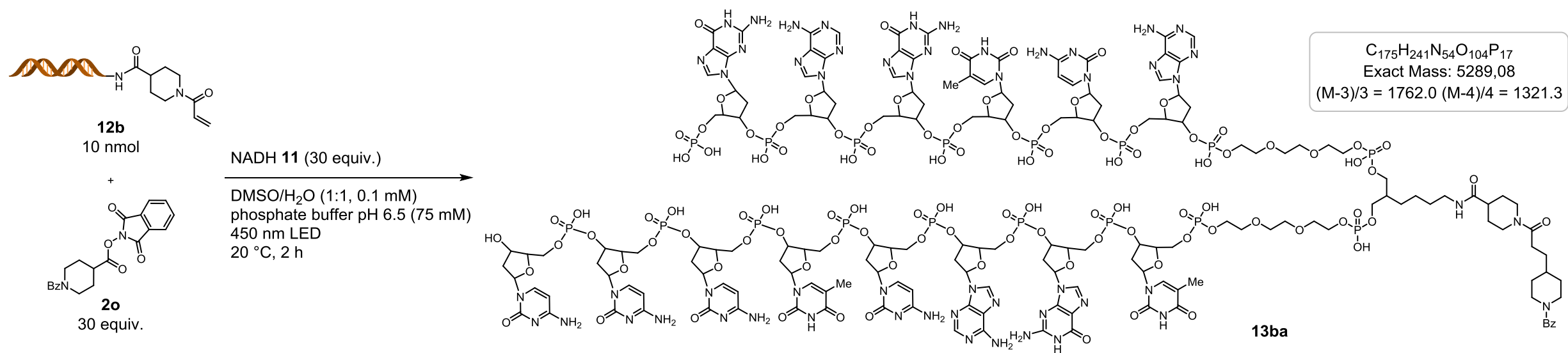

General procedure $\mathbf{C}$ was applied using DNA amide $12 \mathbf{b}\left(20 \mu \mathrm{L}, 0.5 \mathrm{mM}\right.$ in $\mathrm{H}_{2} \mathrm{O}, 10 \mathrm{nmol}, 1.0$ equiv.), redox-active ester $20(10 \mu \mathrm{L}, 30 \mathrm{mM}$ in DMSO, $0.30 \mu \mathrm{mol}, 30$ equiv.), NADH (11; $10 \mu \mathrm{L}, 30 \mathrm{mM}$ in DMSO, $0.30 \mu \mathrm{mol}, 30$ equiv.), and phosphate buffer (30 $\mu \mathrm{L}, 250 \mathrm{mM}, \mathrm{pH} 6.5)$ in DMSO $(30 \mu \mathrm{L})$ for 2 h. Yield: $90 \%$ (6\% s.m.)
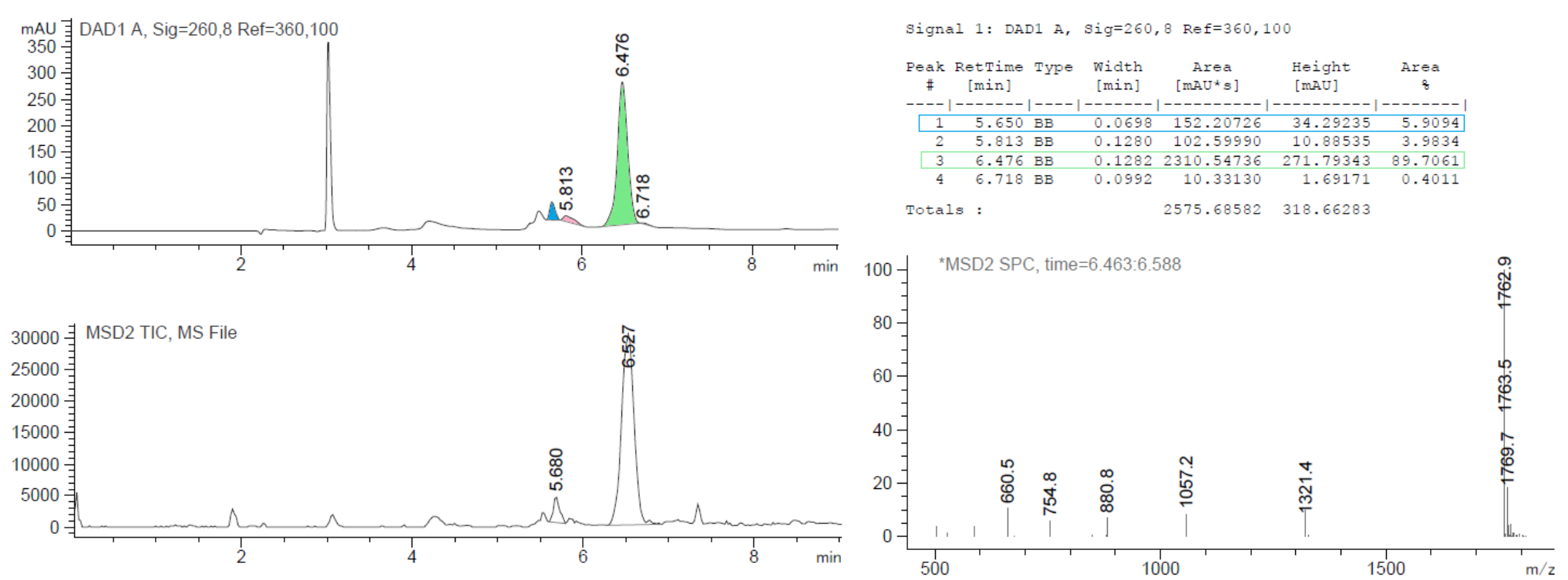

Figure S17: LCMS trace of NADH (11) promoted DNA coupled product 13ba (green) 
Synthesis of DNA functionalized product $13 \mathrm{bb}$ using BuNAH (10) as a reductant

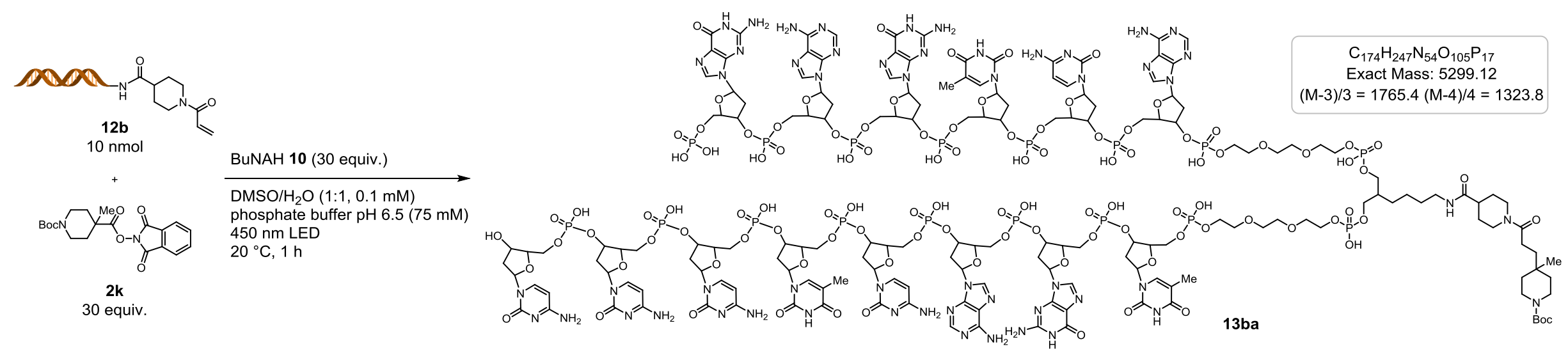

General procedure $C$ was applied using DNA amide $\mathbf{1 2 b}\left(20 \mu \mathrm{L}, 0.5 \mathrm{mM}\right.$ in $\mathrm{H}_{2} \mathrm{O}, 10 \mathrm{nmol}, 1.0$ equiv.), redox-active ester $\mathbf{2 k}(10 \mu \mathrm{L}, 30 \mathrm{mM}$ in DMSO, $0.30 \mu \mathrm{mol}, 30$ equiv.), BuNAH (10; $10 \mu \mathrm{L}, 30 \mathrm{mM}$ in DMSO, $0.30 \mu \mathrm{mol}, 30$ equiv.), and phosphate buffer (30 $\mu \mathrm{L}, 250 \mathrm{mM}, \mathrm{pH} 6.5)$ in DMSO (30 $\mu \mathrm{L})$ for 1 h. Yield: $96 \%$ (0\% s.m.)
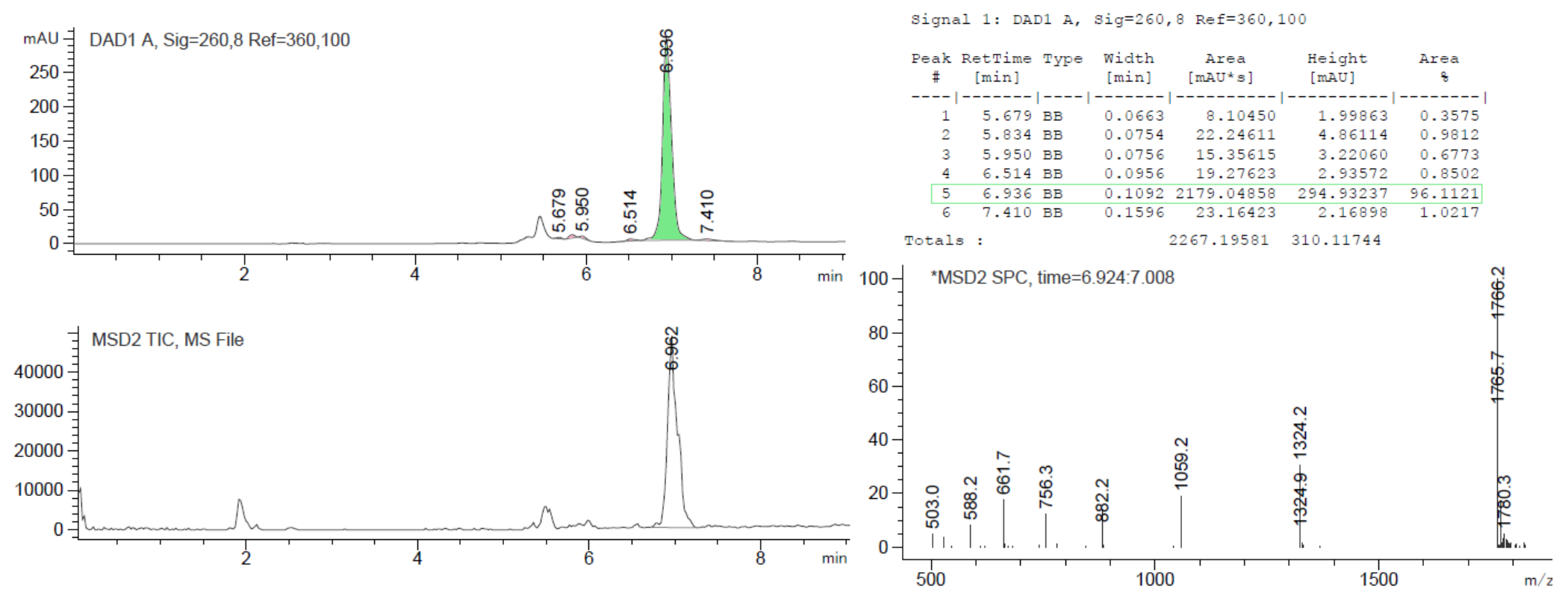

Figure S18: LCMS trace of BUNAH (10) promoted DNA coupled product 13bb (green) 


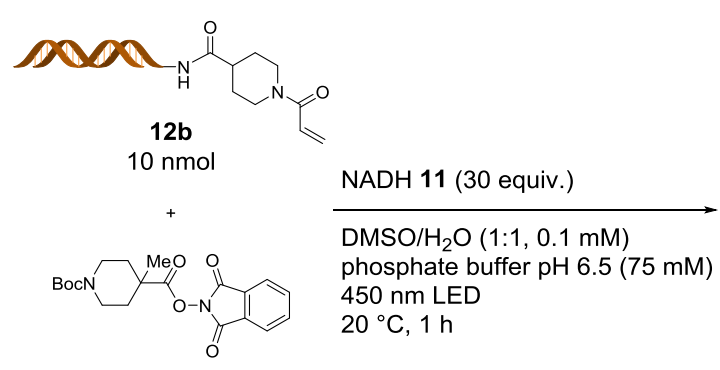

$2 \mathbf{k}$

30 equiv.
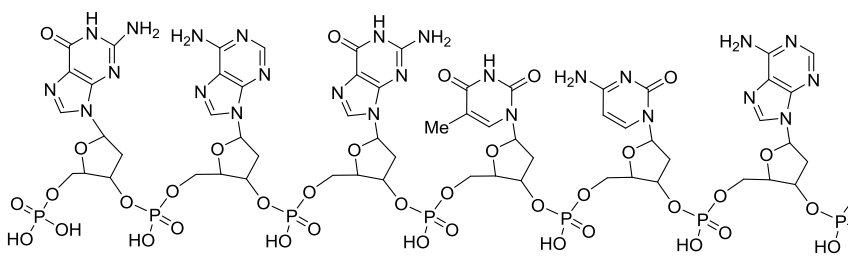

$\mathrm{C}_{174} \mathrm{H}_{247} \mathrm{~N}_{54} \mathrm{O}_{105} \mathrm{P}_{17}$

Exact Mass: 5299.12

$(\mathrm{M}-3) / 3=1765.4(\mathrm{M}-4) / 4=1323.8$

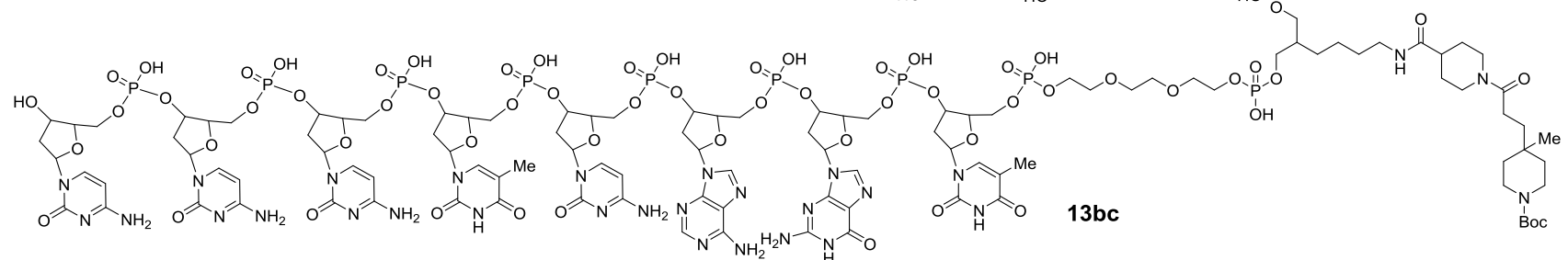

General procedure $C$ was applied using DNA amide $\mathbf{1 2 b}\left(20 \mu \mathrm{L}, 0.5 \mathrm{mM}\right.$ in $\mathrm{H}_{2} \mathrm{O}, 10 \mathrm{nmol}, 1.0$ equiv.), redox-active ester $2 \mathbf{k}(10 \mu \mathrm{L}, 30 \mathrm{mM}$ in DMSO, $0.30 \mu \mathrm{mol}, 30$ equiv.), NADH (11; $10 \mu \mathrm{L}, 30 \mathrm{mM}$ in DMSO, $0.30 \mu \mathrm{mol}, 30$ equiv.), and phosphate buffer (30 $\mu \mathrm{L}, 250 \mathrm{mM}, \mathrm{pH} 6.5)$ in DMSO (30 $\mu \mathrm{L})$ for 1 h. Yield: $93 \%$ (2\% s.m.)

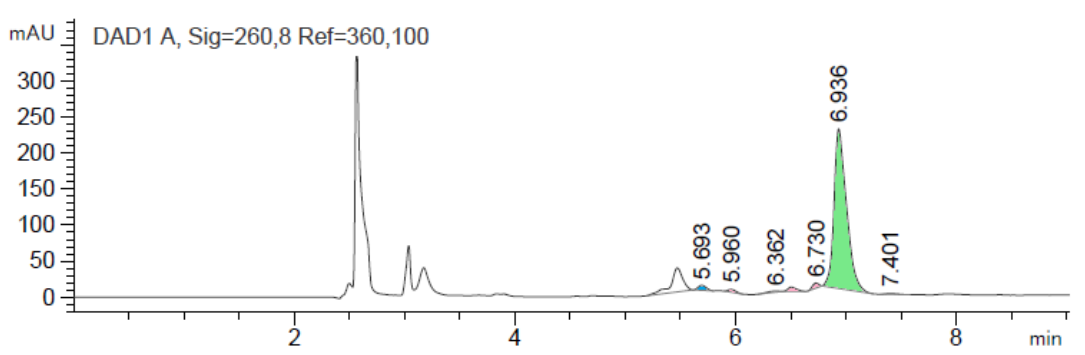

Signal 1: DAD1 A, $\operatorname{Sig}=260,8$ Ref $=360,100$
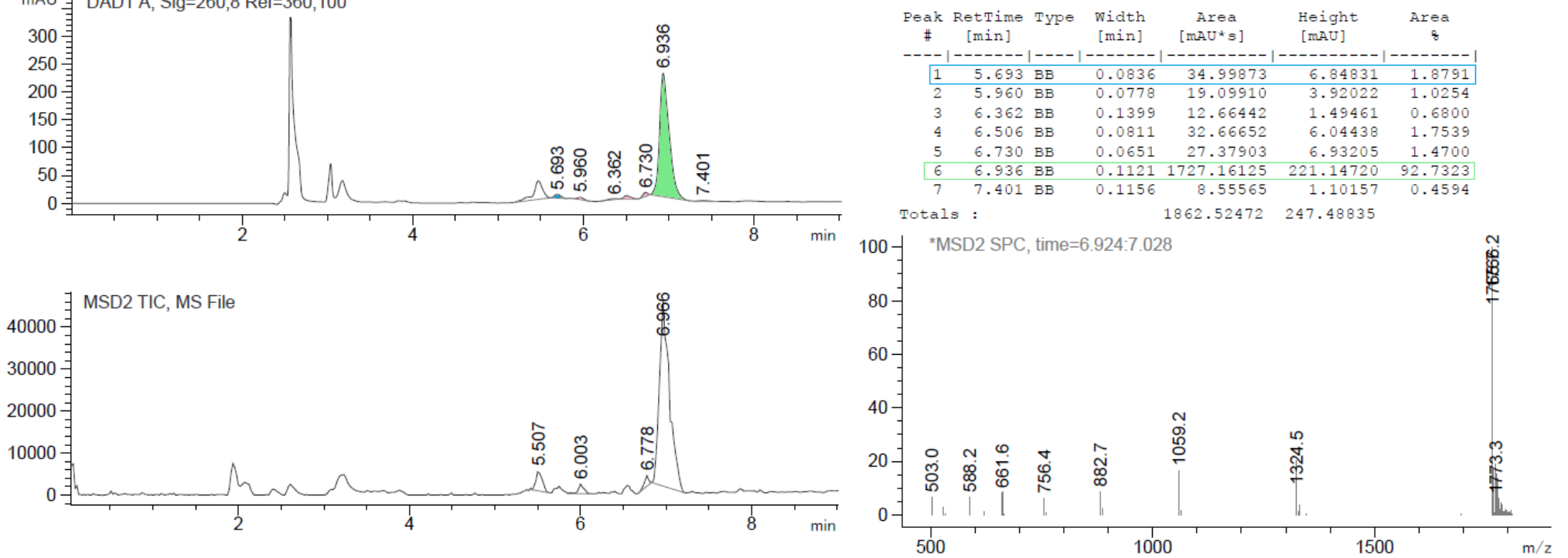

Figure S19: LCMS trace of NADH (11) promoted DNA coupled product 13bb (green) 
Synthesis of DNA functionalized product 13bc using BuNAH (10) as a reductant

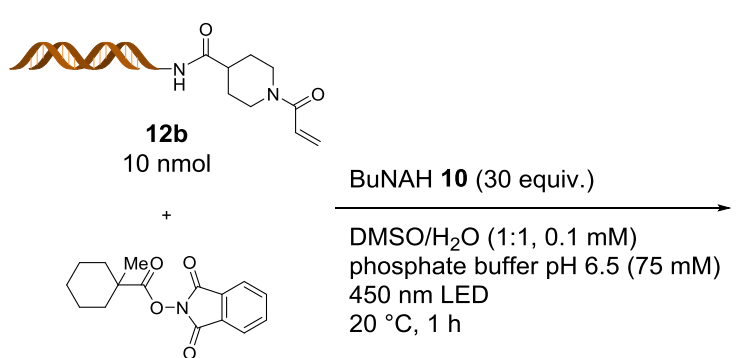

2a equiv.
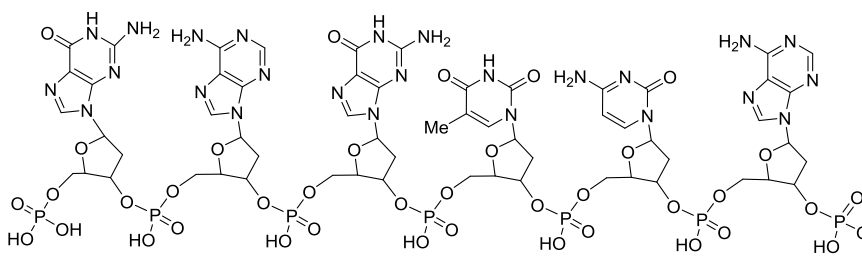

$\mathrm{C}_{170} \mathrm{H}_{240} \mathrm{~N}_{53} \mathrm{O}_{103} \mathrm{P}_{17}$

Exact Mass: 5198.07

$(M-3) / 3=1731.7(M-4) / 4=1298.5$

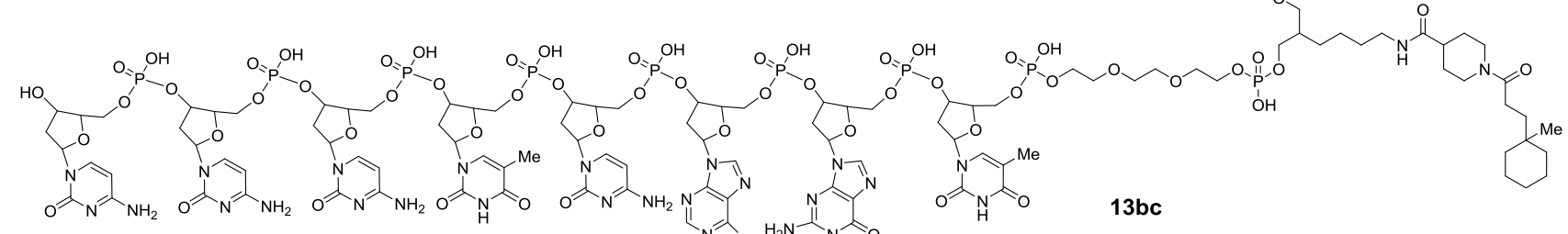

General procedure $C$ was applied using DNA amide $\mathbf{1 2 b}\left(20 \mu \mathrm{L}, 0.5 \mathrm{mM}\right.$ in $\mathrm{H}_{2} \mathrm{O}, 10 \mathrm{nmol}, 1.0$ equiv.), redox-active ester $2 \mathbf{a}(10 \mu \mathrm{L}, 30 \mathrm{mM}$ in DMSO, $0.30 \mu \mathrm{mol}, 30$ equiv.), BuNAH (10; $10 \mu \mathrm{L}, 30 \mathrm{mM}$ in DMSO, $0.30 \mu \mathrm{mol}, 30$ equiv.), and phosphate buffer (30 $\mathrm{LL}, 250 \mathrm{mM}, \mathrm{pH} 6.5)$ in DMSO (30 $\mu \mathrm{L})$ for 1 h. Yield: $97 \%$ (0\% s.m.)

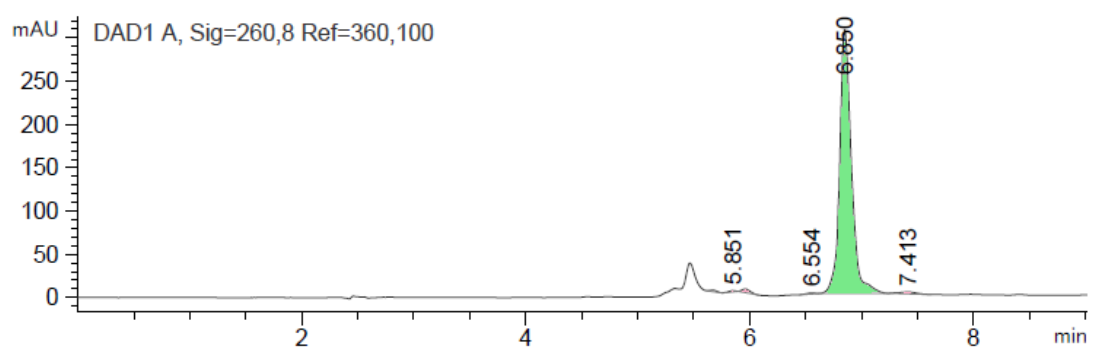

Signal 1: DAD1 A, Sig $=260,8$ Ref $=360,100$
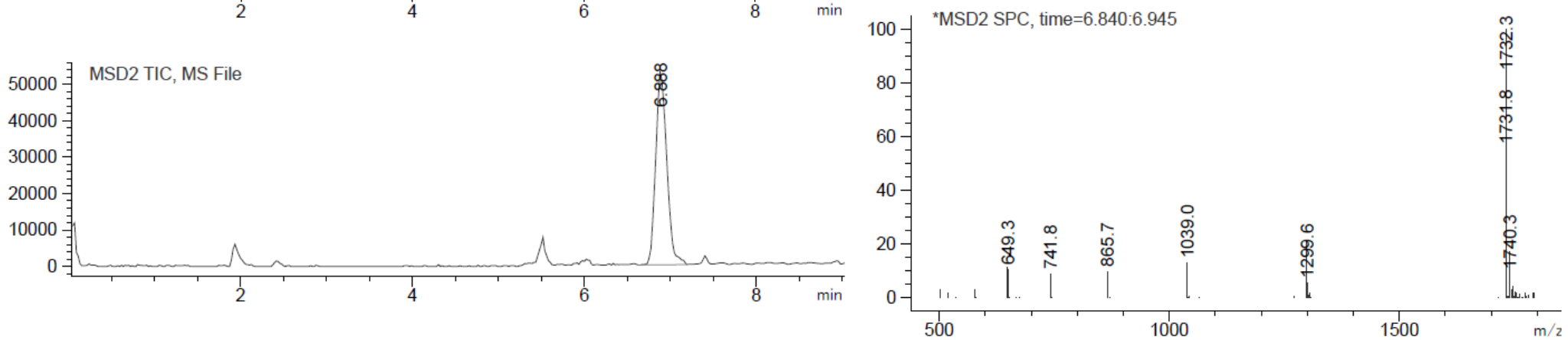

Figure S20: LCMS trace of BuNAH (10) promoted DNA coupled product 13bc (green) 


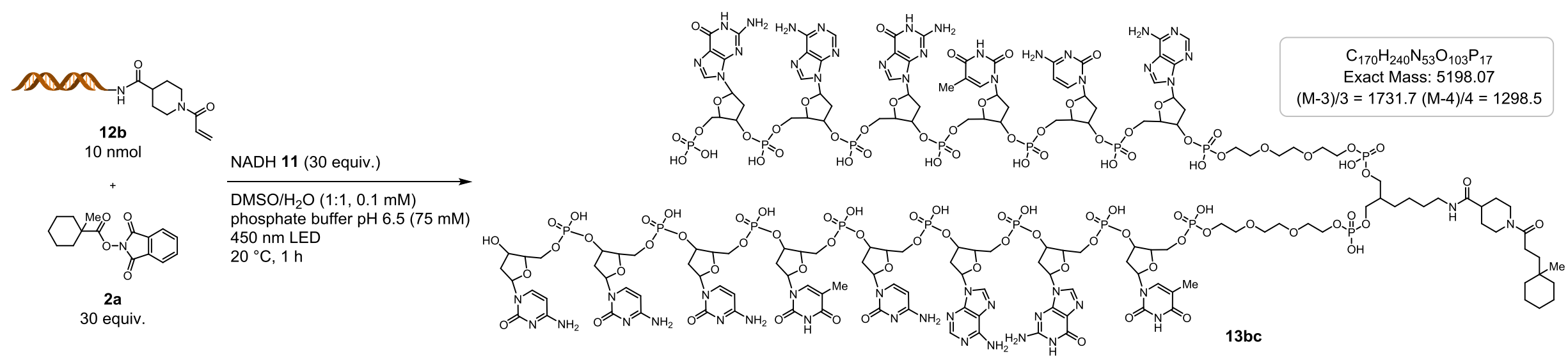

General procedure $\mathrm{C}$ was applied using DNA amide $\mathbf{1 2 b}\left(20 \mu \mathrm{L}, 0.5 \mathrm{mM}\right.$ in $\mathrm{H}_{2} \mathrm{O}, 10 \mathrm{nmol}, 1.0$ equiv.), redox-active ester $2 \mathrm{a}$ (10 $\mu \mathrm{L}, 30 \mathrm{mM}$ in DMSO, $0.30 \mu \mathrm{mol}, 30$ equiv.), NADH (11; $10 \mu \mathrm{L}, 30 \mathrm{mM}$ in DMSO, $0.30 \mu \mathrm{mol}, 30$ equiv.), and phosphate buffer (30 $\mu \mathrm{L}, 250 \mathrm{mM}, \mathrm{pH} 6.5)$ in DMSO (30 $\mu \mathrm{L})$ for 1 h. Yield: $96 \%$ (0\% s.m.)
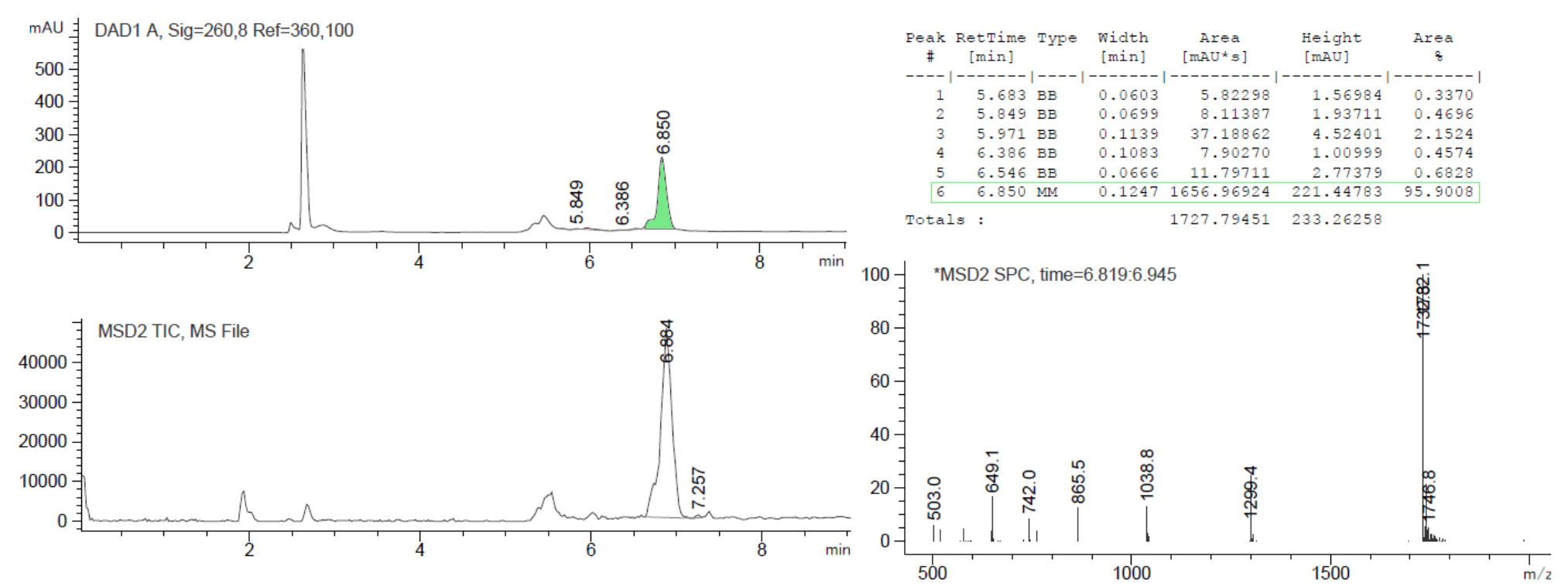

Figure S21: LCMS trace of NADH (11) promoted DNA coupled product 13bc (green) 
Synthesis of DNA functionalized product 13bd using BuNAH (10) as a reductant

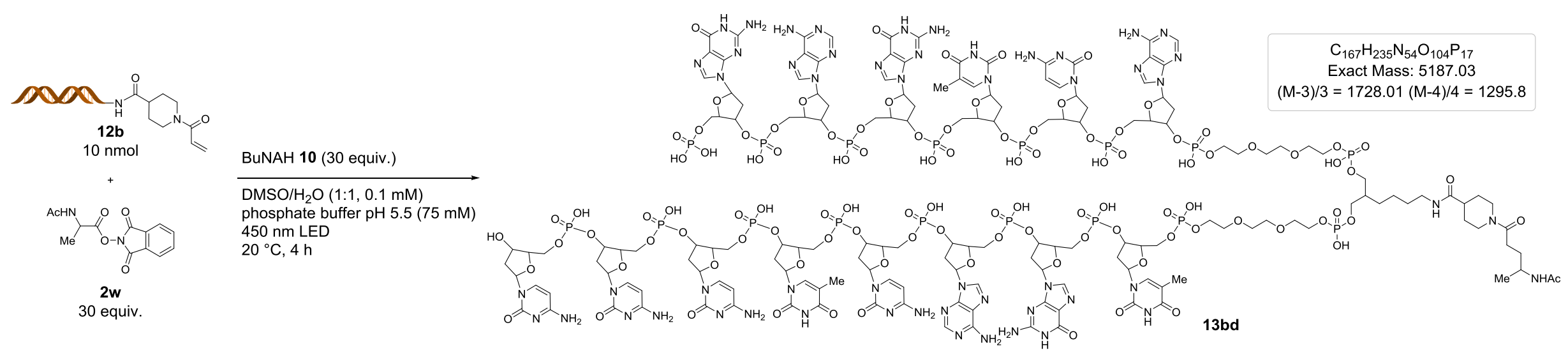

General procedure $\mathrm{C}$ was applied using DNA amide $\mathbf{1 2 b}\left(20 \mu \mathrm{L}, 0.5 \mathrm{mM}\right.$ in $\mathrm{H}_{2} \mathrm{O}, 10 \mathrm{nmol}, 1.0$ equiv.), redox-active ester $2 \mathbf{w}$ (10 $\mu \mathrm{L}, 30 \mathrm{mM}$ in DMSO, $0.30 \mu \mathrm{mol}, 30$ equiv.), BuNAH (10; $10 \mu \mathrm{L}, 30 \mathrm{mM}$ in DMSO, $0.30 \mu \mathrm{mol}, 30$ equiv.) in DMSO (30 $\mu \mathrm{L})$, and phosphate buffer (30 $\mu \mathrm{L}$, $250 \mathrm{mM}$, pH 5.5) for 4 hours. Yield: n.d.
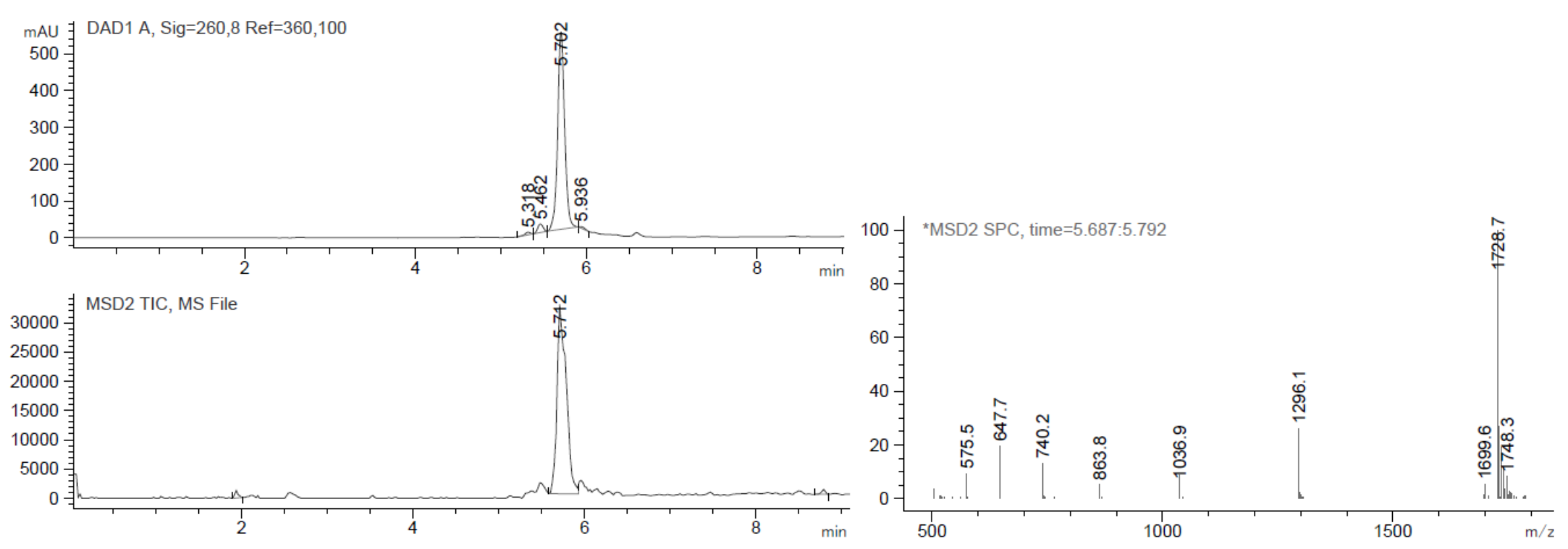

Figure S22: LCMS trace of BUNAH (10) promoted DNA coupled product 13bd. 

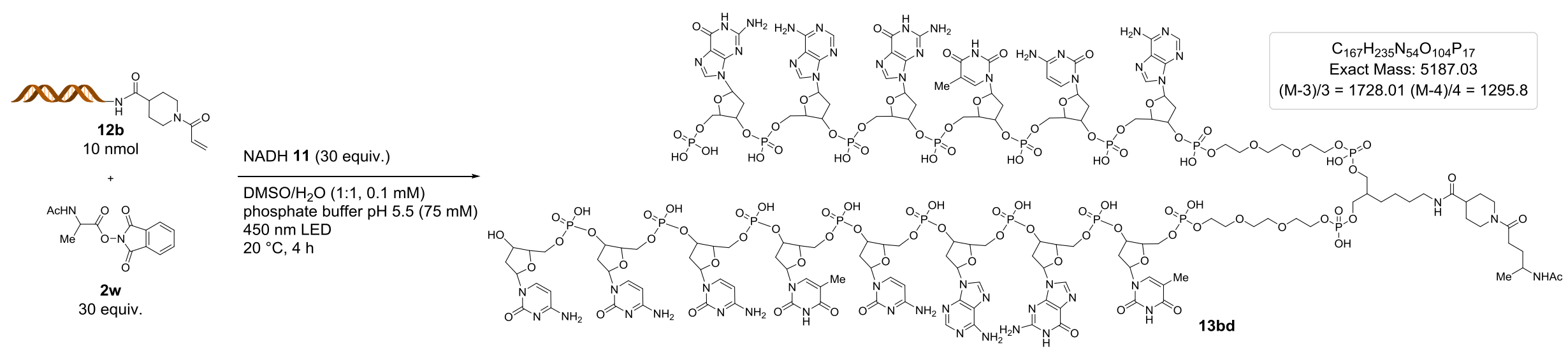

General procedure $\mathrm{C}$ was applied using DNA acrylate $\mathbf{1 2 b}\left(20 \mu \mathrm{L}, 0.5 \mathrm{mM}\right.$ in $\mathrm{H}_{2} \mathrm{O}, 10 \mathrm{nmol}, 1.0$ equiv.), redox-active ester (2v; $10 \mu \mathrm{L}, 30 \mathrm{mM}$ in DMSO, $0.30 \mu \mathrm{mol}, 30$ equiv.), NADH (11; $10 \mu \mathrm{L}, 30 \mathrm{mM}$ in DMSO, $0.30 \mu \mathrm{mol}, 30$ equiv.) in DMSO (30 $\mu \mathrm{L})$, and phosphate buffer (30 $\mu \mathrm{L}$, $250 \mathrm{mM}$, pH 6.5) for 4 hours. Yield: n.d.
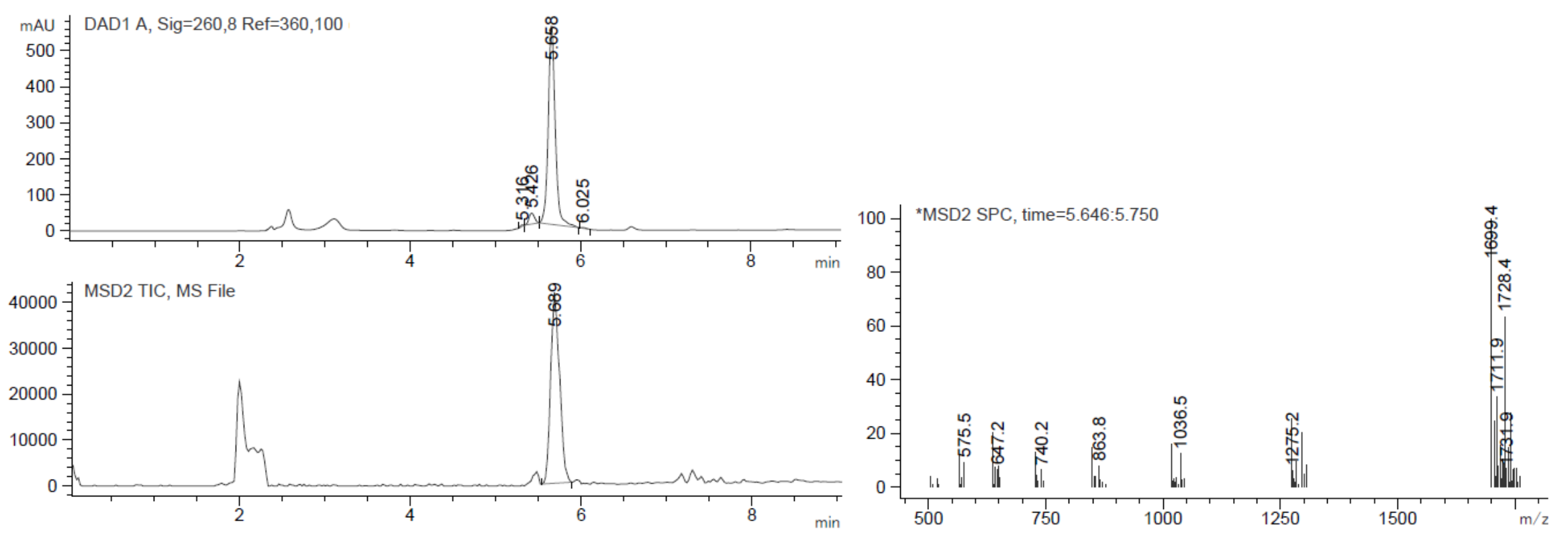

Figure S23: LCMS trace of NADH (10) promoted DNA coupled product $\mathbf{1 3 b d}$. 


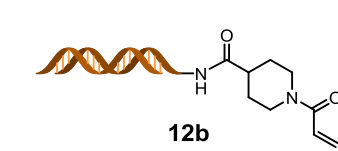

$10 \mathrm{nmol}$

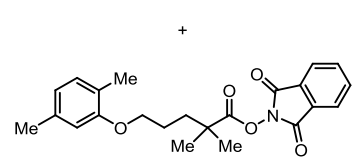

$2 \mathrm{~m}$
30 equiv.

\section{BUNAH 10 (30 equiv.)}

$\mathrm{DMSO} / \mathrm{H}_{2} \mathrm{O}(1: 1,0.1 \mathrm{mM})$

phosphate buffer $\mathrm{pH} 6.5(75 \mathrm{mM})$ $450 \mathrm{~nm}$ LED
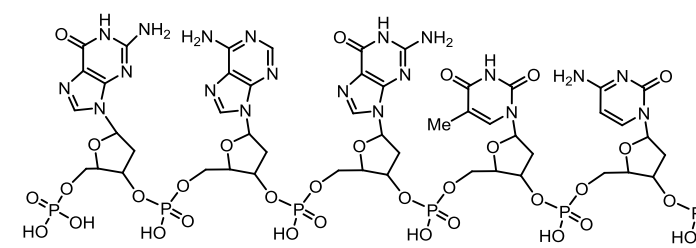

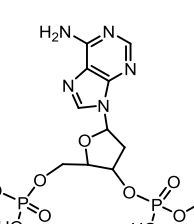

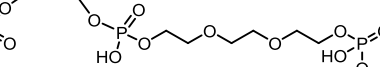

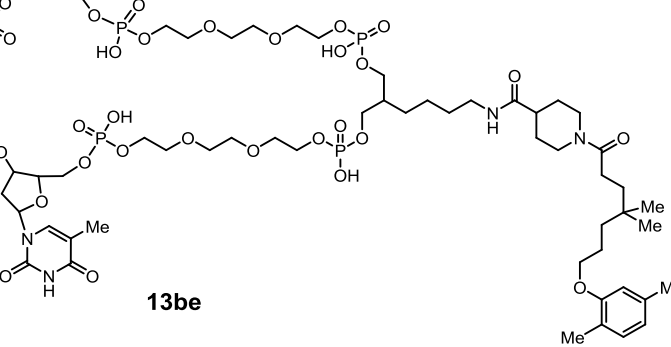

General procedure $C$ was applied using DNA amide $12 \mathbf{b}\left(20 \mu \mathrm{L}, 0.5 \mathrm{mM}\right.$ in $\mathrm{H}_{2} \mathrm{O}, 10 \mathrm{nmol}, 1.0$ equiv.), redox-active ester $2 \mathrm{~m}(10 \mu \mathrm{L}, 30 \mathrm{mM}$ in DMSO, $0.30 \mu \mathrm{mol}, 30$ equiv.), BuNAH (10; $10 \mu \mathrm{L}, 30 \mathrm{mM}$ in DMSO, $0.30 \mu \mathrm{mol}, 30$ equiv.), and phosphate buffer (30 $\mu \mathrm{L}, 250 \mathrm{mM}, \mathrm{pH} 6.5)$ in DMSO (30 $\mu \mathrm{L})$ for $1 \mathrm{~h}$. Yield: $97 \%$ (0\% s.m.)
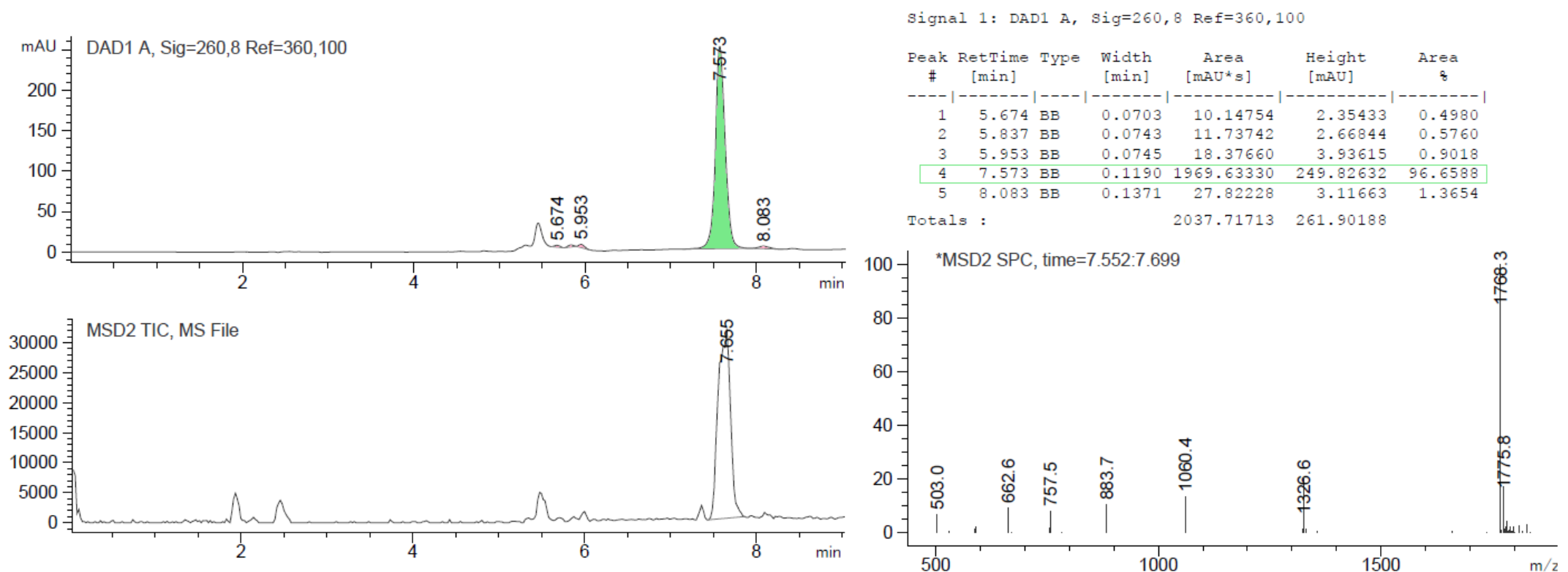

Figure S24: LCMS trace of BuNAH (10) promoted DNA coupled product 13be (green) 
Synthesis of DNA functionalized product 13be using NADH (11) as a reductant
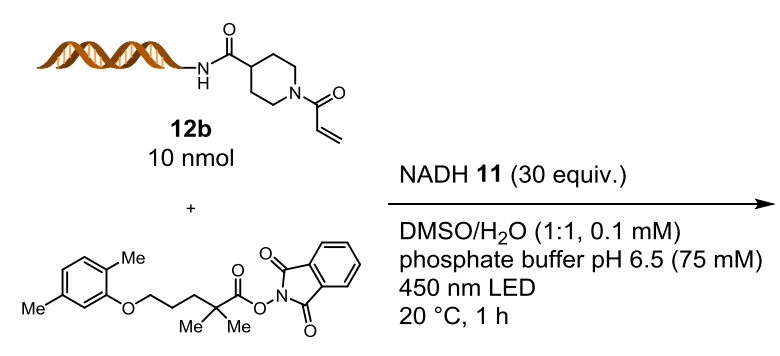

$2 \mathrm{~m}$

30 equiv.

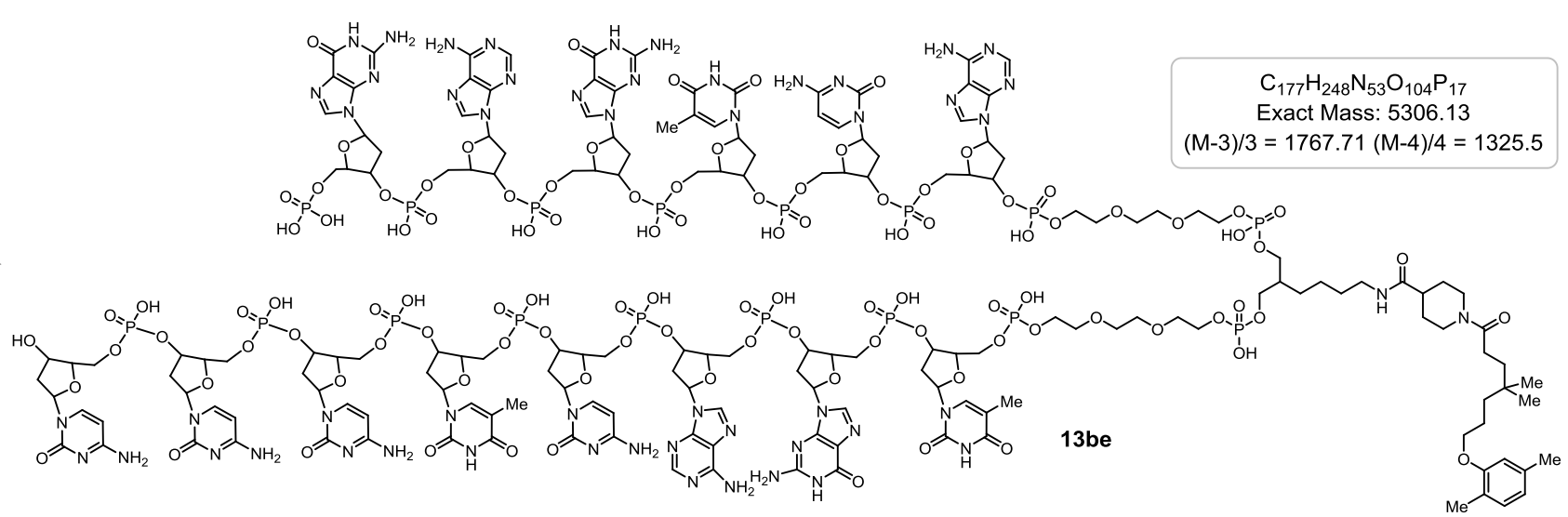

General procedure $\mathrm{C}$ was applied using DNA amide $12 \mathrm{~b}\left(20 \mu \mathrm{L}, 0.5 \mathrm{mM}\right.$ in $\mathrm{H}_{2} \mathrm{O}, 10 \mathrm{nmol}, 1.0$ equiv.), redox-active ester 20 (10 $\mu \mathrm{L}$, $30 \mathrm{mM}$ in DMSO, $0.30 \mu \mathrm{mol}, 30$ equiv.), NADH (11; $10 \mu \mathrm{L}, 30 \mathrm{mM}$ in DMSO, $0.30 \mu \mathrm{mol}, 30$ equiv.), and phosphate buffer (30 $\mu \mathrm{L}, 250 \mathrm{mM}, \mathrm{pH} 6.5)$ in DMSO (30 $\mu$ L) for 1 h. Yield: $74 \%$ (20\% s.m.)
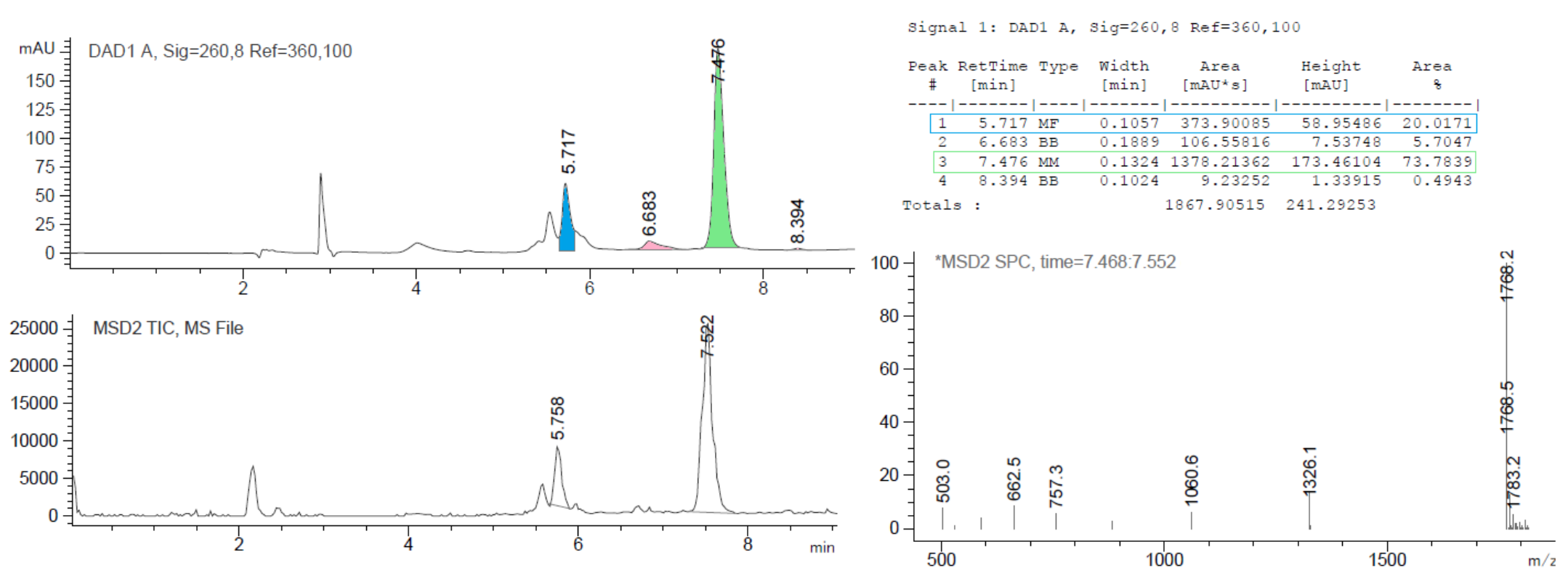

Figure S25: LCMS trace of NADH (11) promoted DNA coupled product 3be (green) 


\section{Kinetic profiling by in situ NMR experiments}

\section{Procedure for the in situ NMR experiment under standard reaction conditions (no-D NMR)}

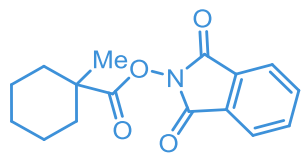

$2 a$ $0.05 \mathrm{mmol}$

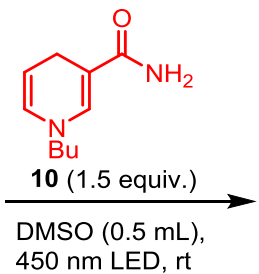

$3 a$ 1.5 equiv.

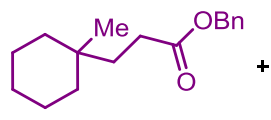

4a

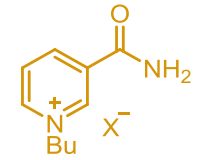

18

The reaction sample was prepared in a NMR tube under inert gas atmosphere in the dark. 1,3-dioxoisoindolin-2-yl 1-methylcyclohexane-1-carboxylate (2a; $14 \mathrm{mg}, 50 \mu \mathrm{mol}, 1.0$ equiv.) was dissolved in DMSO (0.1 mL), and benzyl acrylate (3a; $11 \mu \mathrm{L}, 75 \mu \mathrm{mol}, 1.5$ equiv.), and 1butyl-1,4-dihydropyridine-3-carboxamide (10; $0.4 \mathrm{~mL}$ in DMSO, $0.2 \mathrm{M}, 75 \mu \mathrm{mol}, 1.5$ equiv.) were subsequently added. The reaction mixture was illuminated with blue LED at $20-22{ }^{\circ} \mathrm{C}$, and progress of the reaction was followed by no-D NMR.

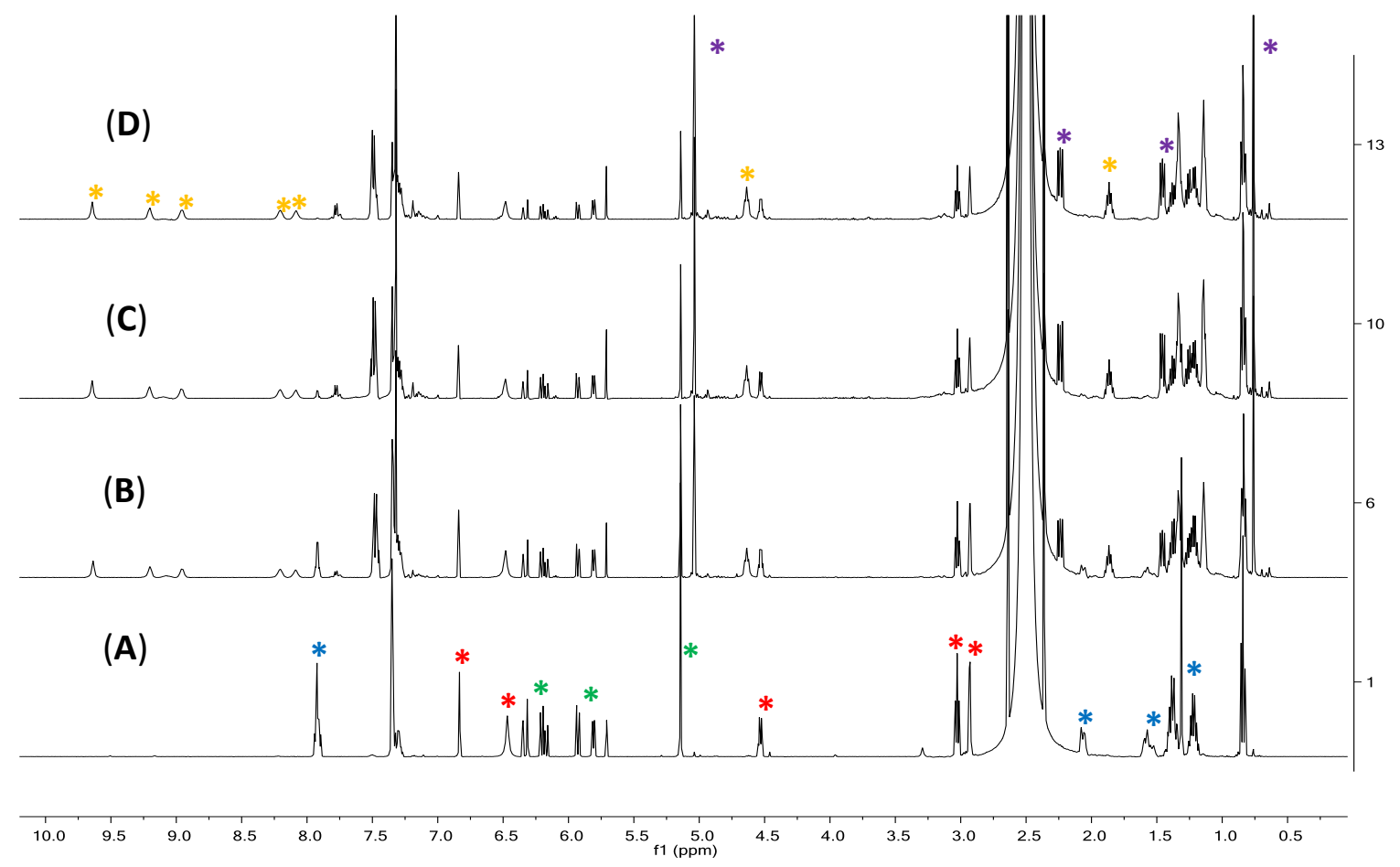

Figure S26: ${ }^{1} \mathrm{H}-N M R$ spectra after (A) O s, (B) $100 \mathrm{~s},(C) 180 \mathrm{~s} ;(D) 240$ s irradiation in DMSO. 


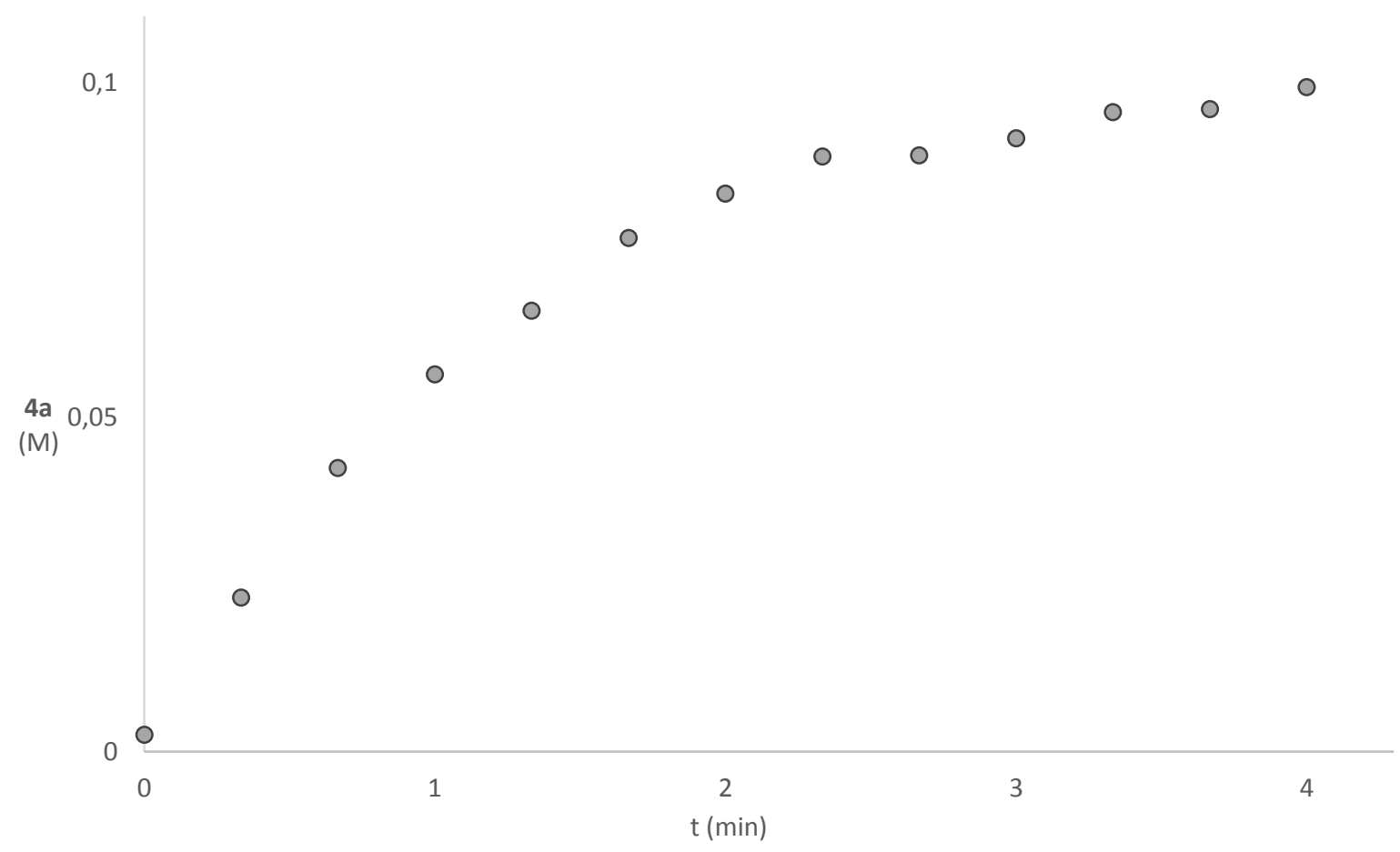

Figure S27: Kinetic profiling of photo-ligation under standard reaction conditions. (see.

\section{Table S4)}

Table S4: Data of progress of photo-ligation reaction under standard reaction condition. (see.

Figure 527

\begin{tabular}{|c|c|c|c|}
\hline Entry & Time $(\mathrm{s})$ & Conversion 2a (\%) & [4a] (M) \\
\hline 1 & 0 & 0 & 0.002 \\
\hline 2 & 20 & 22 & 0.023 \\
\hline 3 & 40 & 46 & 0.042 \\
\hline 4 & 60 & 59 & 0.056 \\
\hline 5 & 80 & 70 & 0.066 \\
\hline 6 & 100 & 77 & 0.077 \\
\hline 7 & 120 & 83 & 0.083 \\
\hline 8 & 140 & 88 & 0.089 \\
\hline 9 & 160 & 93 & 0.089 \\
\hline 10 & 180 & 94 & 0.092 \\
\hline 11 & 200 & 97 & 0.096 \\
\hline 12 & 220 & 97 & 0.096 \\
\hline 13 & 240 & 97 & 0.099 \\
\hline
\end{tabular}

Reaction conditions: $2 \mathrm{a}(0.1 \mathrm{M}), \operatorname{BuNAH}(\mathbf{1 0} ; 0.15 \mathrm{M})$ and benzyl acrylate (3a; $0.15 \mathrm{M})$ in DMSO $(0.5 \mathrm{~mL})$, irradiation with blue LEDs at $20-22^{\circ} \mathrm{C}$. 


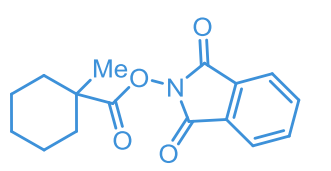

2a $0.05 \mathrm{mmol}$

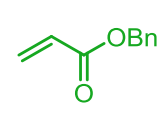

$3 a$ 1.5 equiv.<smiles>NC(=O)C1=CNC=CC1</smiles>

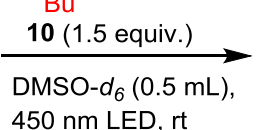

$450 \mathrm{~nm}$ LED, $\mathrm{rt}$<smiles>CC1(CCC(=O)OCc2ccccc2)CCCCC1</smiles>

$4 a$

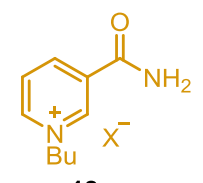

18

To a solution of 1,3-dioxoisoindolin-2-yl 1-methylcyclohexane-1-carboxylate (2a; $14 \mathrm{mg}, 50$ $\mu \mathrm{mol}, 1.0$ equiv.) in DMSO- $d_{6}(0.1 \mathrm{~mL})$, in a NMR tube was added benzyl acrylate (3a; $11 \mu \mathrm{L}$, $75 \mu \mathrm{mol}, 1.5$ equiv.) and 1-butyl-1,4-dihydropyridine-3-carboxamide (10; $0.4 \mathrm{~mL}$ in DMSO- $d_{6}$, $0.2 \mathrm{M}, 75 \mu \mathrm{mol}, 1.5$ equiv.). Preparation of the reaction sample was done in the dark under argon. The sample was illuminated with blue LED at $20-22^{\circ} \mathrm{C}$ and the progress of the reaction was followed by ${ }^{1} \mathrm{H}-\mathrm{NMR}$.

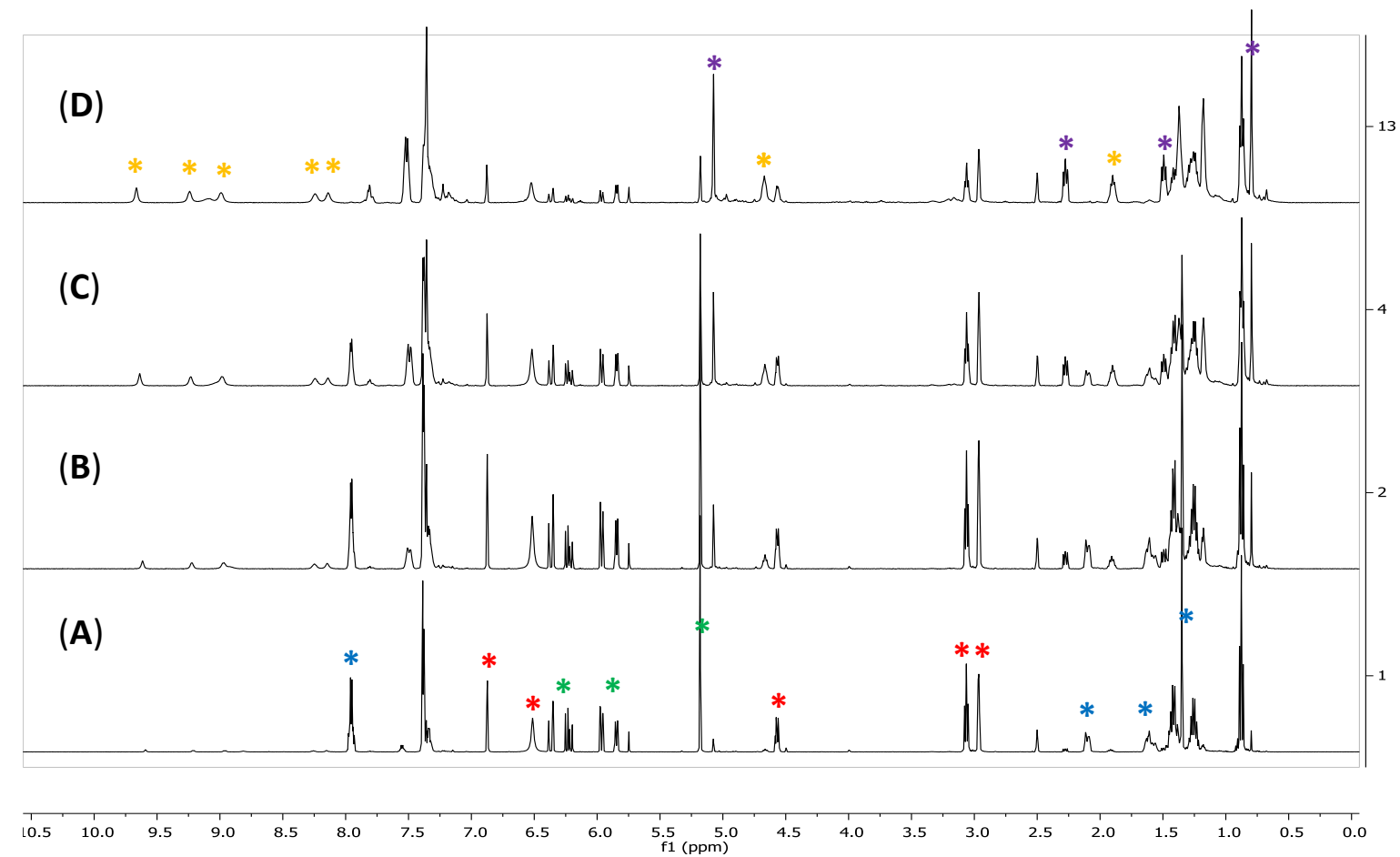

Figure S28: ${ }^{1} \mathrm{H}-\mathrm{NMR}$ spectra after (A) $\left.0 \mathrm{~s},(B) 20 \mathrm{~s}, C\right) 60 \mathrm{~s},(D) 240 \mathrm{~s}$ irradiation in DMSO-d 6 


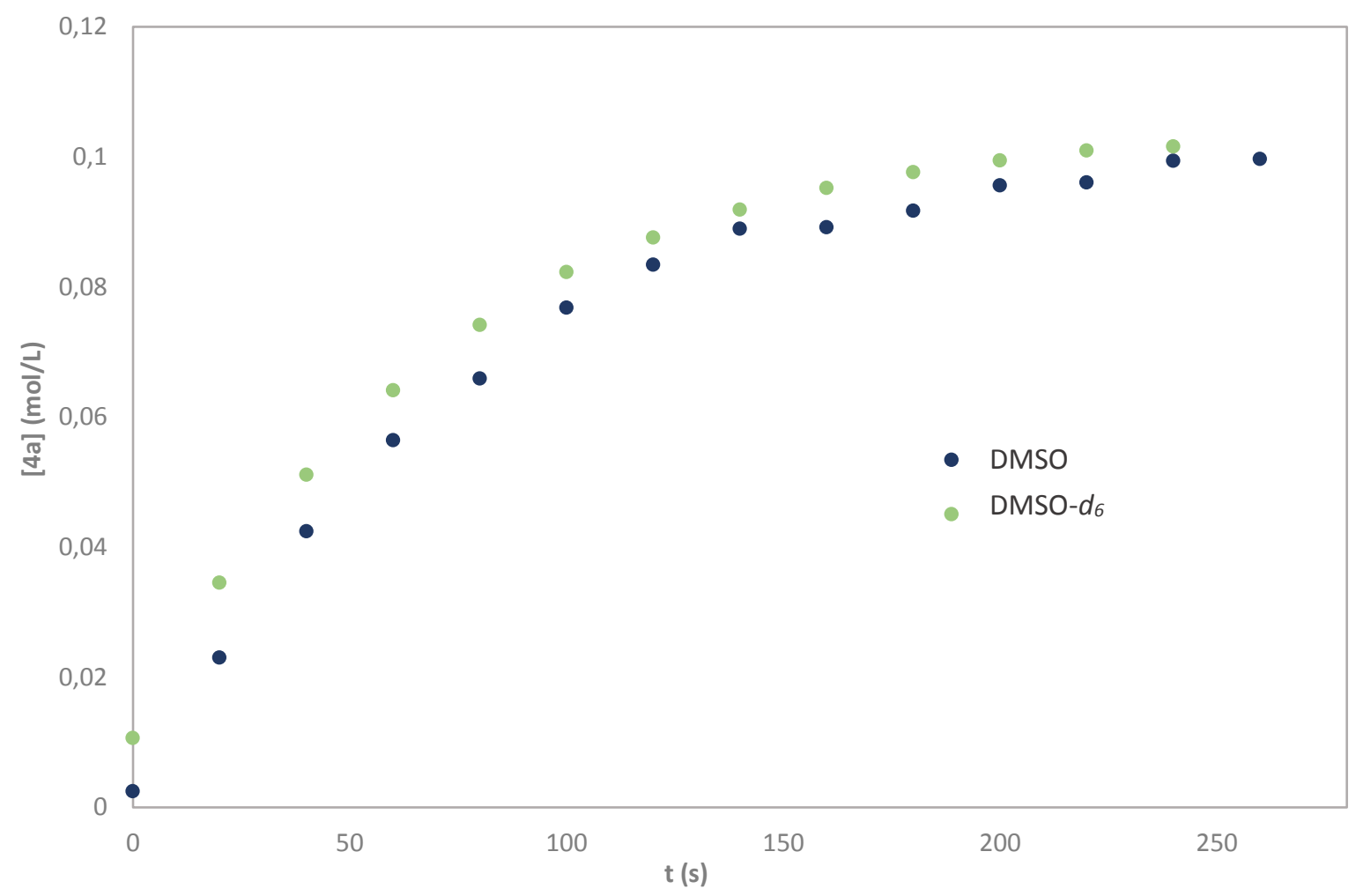

Figure S29: Overlay of the standard reaction in DMSO (see. Figure S27) and DMSO-d 6 (see. Table S5) 


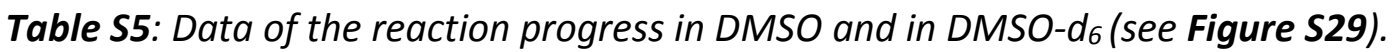

\begin{tabular}{|c|c|c|c|c|}
\hline Entry & \multicolumn{3}{|c|}{ DMSO } & \multicolumn{2}{c|}{ DMSO- $d_{6}$} \\
\hline & Time $(s)$ & {$[\mathbf{4 a}](\mathbf{M})$} & Time $(\mathrm{s})$ & {$[\mathbf{4 a}](\mathrm{M})$} \\
\hline 1 & 0 & 0.002 & 0 & 0.010 \\
\hline 2 & 20 & 0.023 & 20 & 0.035 \\
\hline 3 & 40 & 0.042 & 40 & 0.051 \\
\hline 4 & 60 & 0.056 & 60 & 0.064 \\
\hline 5 & 80 & 0.066 & 80 & 0.074 \\
\hline 6 & 100 & 0.077 & 100 & 0.082 \\
\hline 7 & 120 & 0.083 & 120 & 0.088 \\
\hline 8 & 140 & 0.089 & 140 & 0.092 \\
\hline 9 & 160 & 0.089 & 160 & 0.095 \\
\hline 10 & 180 & 0.092 & 180 & 0.098 \\
\hline 11 & 200 & 0.096 & 200 & 0.100 \\
\hline 13 & 220 & 0.096 & 220 & 0.100 \\
\hline
\end{tabular}

Reaction conditions: $2 a(0.1 \mathrm{M})$, BuNAH $(10 ; 0.15 \mathrm{M})$ and benzyl acrylate $(3 \mathrm{a} ; 0.15 \mathrm{M})$ in solvent $(0.5 \mathrm{~mL})$, irradiation with blue LEDs at $20-22{ }^{\circ} \mathrm{C}$.

Procedure for the in situ NMR experiment to profile the background and deactivation of the system
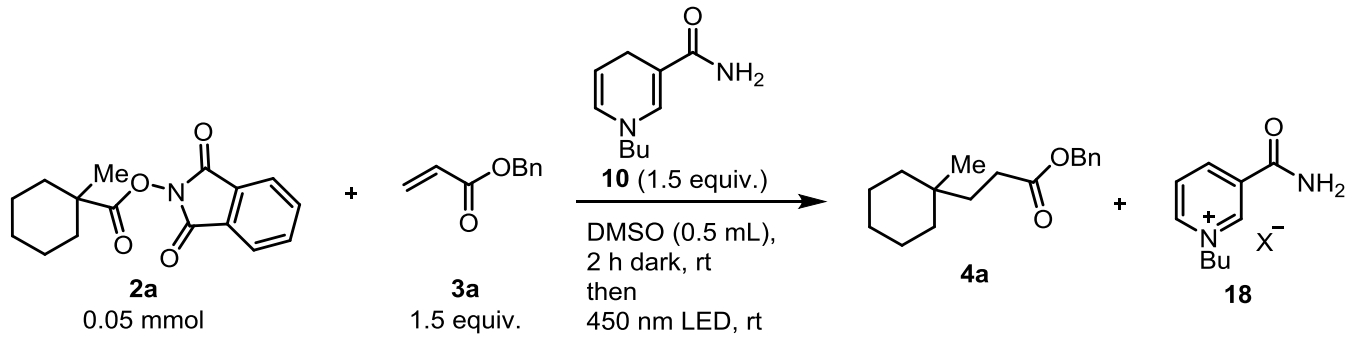

To a solution of 1,3-dioxoisoindolin-2-yl 1-methylcyclohexane-1-carboxylate (2a; $14 \mathrm{mg}, 50$ $\mu \mathrm{mol}, 1.0$ equiv.) in DMSO (0.1 mL), benzyl acrylate (3a; $11 \mu \mathrm{L}, 75 \mu \mathrm{mol}, 1.5$ equiv.), and 1butyl-1,4-dihydropyridine-3-carboxamide (10; $0.4 \mathrm{~mL}$ in DMSO, $0.2 \mathrm{M}, 75 \mu \mathrm{mol}, 1.5$ equiv.) were added in the dark under argon. The sample was kept at $20^{\circ} \mathrm{C}$ for $2 \mathrm{~h}$ during which it was monitored by ${ }^{1} \mathrm{H}-\mathrm{NMR}$ under strict exclusion of light. Then, the sample was illuminated at 20-22 ${ }^{\circ} \mathrm{C}$ with blue LEDs, and the progress of the reaction was followed by no-D ${ }^{1} \mathrm{H}-\mathrm{NMR}$. 


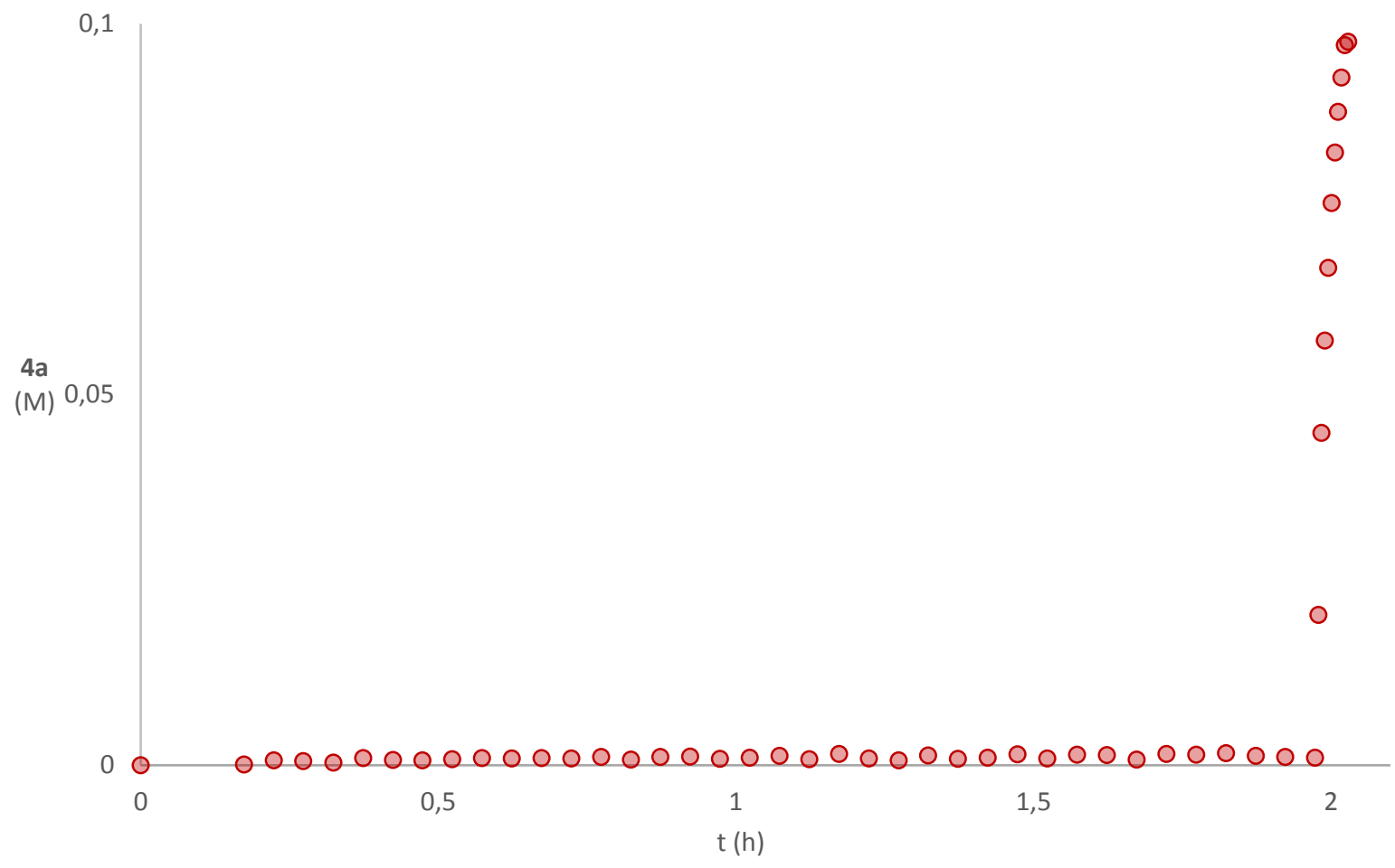

Figure S30: Reaction profile of the formation of $\mathbf{4 a}$ in the absence of light and during the illumination period after $2 \mathrm{~h}$ of dark pre-treatment (see Table S6). 
Table S6: Data of the formation of 4a in the absence of light and during the illumination period after $2 \mathrm{~h}$ of dark pre-treatment.(see. Figure S30)

\begin{tabular}{|c|c|c|c|c|c|}
\hline Entry & Time (s) & [4a] (M) & Entry & Time (s) & [4a] (M) \\
\hline 1 & 0 & 0.000 & 28 & 4764 & 0.001 \\
\hline 2 & 85 & 0.000 & 29 & 4944 & 0.001 \\
\hline 3 & 265 & 0.000 & 30 & 5124 & 0.001 \\
\hline 4 & 445 & 0.000 & 31 & 5304 & 0.001 \\
\hline 5 & 624 & 0.000 & 32 & 5485 & 0.001 \\
\hline 6 & 804 & 0.001 & 33 & 5665 & 0.001 \\
\hline 7 & 984 & 0.001 & 34 & 5845 & 0.001 \\
\hline 8 & 1165 & 0.000 & 35 & 6025 & 0.001 \\
\hline 9 & 1345 & 0.001 & 36 & 6205 & 0.002 \\
\hline 10 & 1525 & 0.001 & 37 & 6385 & 0.001 \\
\hline 11 & 1705 & 0.001 & 38 & 6565 & 0.001 \\
\hline 12 & 1885 & 0.001 & 39 & 6745 & 0.001 \\
\hline 13 & 2065 & 0.001 & 40 & 6925 & 0.001 \\
\hline 14 & 2245 & 0.001 & 41 & 7104 & 0.001 \\
\hline 15 & 2425 & 0.001 & 42 & 7124 & 0.020 \\
\hline 16 & 2605 & 0.001 & 43 & 7144 & 0.045 \\
\hline 17 & 2785 & 0.001 & 44 & 7164 & 0.057 \\
\hline 18 & 2965 & 0.001 & 45 & 7184 & 0.067 \\
\hline 19 & 3144 & 0.001 & 46 & 7204 & 0.076 \\
\hline 20 & 3324 & 0.001 & 47 & 7224 & 0.083 \\
\hline 21 & 3504 & 0.001 & 48 & 7244 & 0.088 \\
\hline 22 & 3684 & 0.001 & 49 & 7264 & 0.093 \\
\hline 23 & 3865 & 0.001 & 50 & 7284 & 0.097 \\
\hline 24 & 4045 & 0.001 & 51 & 7304 & 0.098 \\
\hline 25 & 4225 & 0.002 & 52 & 7324 & 0.100 \\
\hline 26 & 4405 & 0.001 & 53 & 7344 & 0.101 \\
\hline 27 & 4585 & 0.001 & 54 & 7364 & 0.101 \\
\hline
\end{tabular}

Conditions: 2a (0.1 M), BuNAH (10; $0.15 \mathrm{M})$ and benzyl acrylate (3a; $0.15 \mathrm{M})$ in DMSO (0.5 $\mathrm{mL})$. 


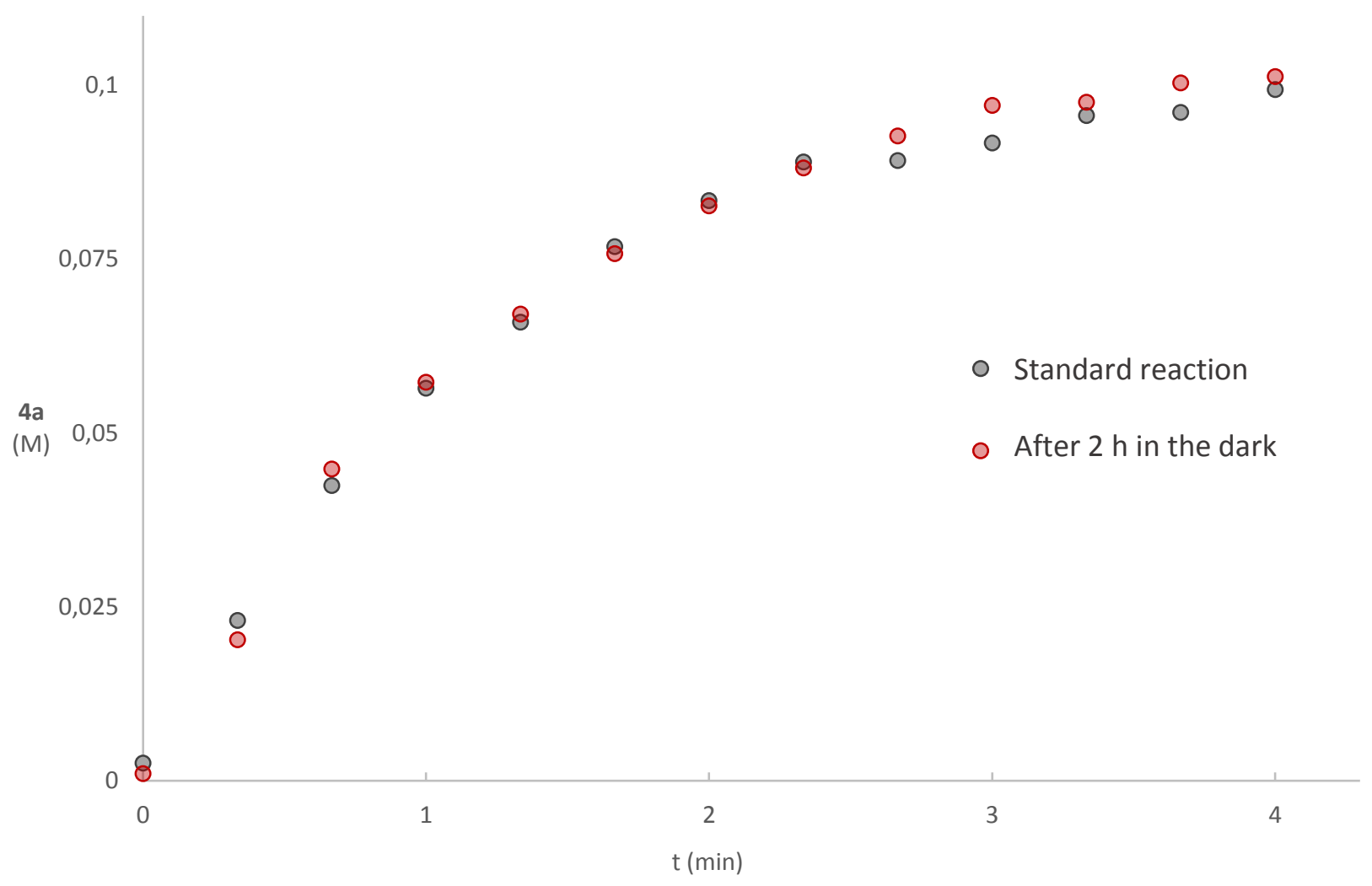

Figure S31: Overlay of the reaction profiles of the standard reaction (see. Figure S27) and the reaction after $2 \mathrm{~h}$ in the dark (see. Figure S30). Time has been normalized to the period of illumination. (see. Table S7) 
Table S7: Time normalized data of the illumination periods in the standard reaction (See:

Table S4) and after 2 h dark pre-treatment (see Table S6). See Figure S31

\begin{tabular}{|c|c|c|c|c|}
\hline & \multicolumn{2}{|c|}{ Standard condition } & \multicolumn{2}{c|}{ After $2 \mathrm{~h}$ in dark } \\
\hline Entry & Time $(\mathrm{s})$ & {$[\mathbf{4 a}] \mathbf{( M )}$} & Time $(\mathrm{s})$ & $[\mathbf{4 a}] \mathbf{M})$ \\
\hline 1 & 0 & 0.002 & 0 & 0.001 \\
\hline 2 & 20 & 0.023 & 20 & 0.020 \\
\hline 3 & 40 & 0.042 & 40 & 0.045 \\
\hline 4 & 60 & 0.056 & 60 & 0.057 \\
\hline 5 & 80 & 0.066 & 80 & 0.067 \\
\hline 6 & 100 & 0.077 & 100 & 0.076 \\
\hline 7 & 120 & 0.083 & 120 & 0.083 \\
\hline 8 & 140 & 0.089 & 140 & 0.088 \\
\hline 9 & 160 & 0.089 & 160 & 0.093 \\
\hline 10 & 180 & 0.092 & 180 & 0.097 \\
\hline 11 & 200 & 0.096 & 200 & 0.098 \\
\hline 12 & 220 & 0.096 & 220 & 0.100 \\
\hline 13 & 240 & 0.099 & 240 & 0.101 \\
\hline
\end{tabular}

Standard condition: 2a (0.1 M), BuNAH (10;0.15 M) and benzyl acrylate (3a; $0.15 \mathrm{M})$ in DMSO $(0.5 \mathrm{~mL})$, irradiation with blue LEDs at $20-22^{\circ} \mathrm{C}$.

Procedure for the in situ NMR experiment to study the kinetic effect of the initial concentration of $2 a$<smiles>[M]C1(C(=O)ON2C(=O)c3ccccc3C2=O)CCCCC1</smiles>

$2 a$

(1.0 equiv.)<smiles>C=CC(=O)OCc1ccccc1</smiles>

$3 a$ $(1.5$ equiv. $)$<smiles>NC(=O)c1cccnc1</smiles>

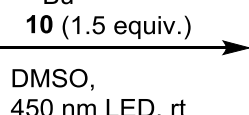

$450 \mathrm{~nm}$ LED, rt<smiles>CC1(CCC(=O)OCc2ccccc2)CCCCC1</smiles>

$4 a$<smiles>[Y][14c]1[14cH]cccc1C(N)=O</smiles>

An oven-dried NMR tube was charged with the appropriate amount of stock solutions of $2 a$ (1.0 equiv.), BuNAH (10; 1.5 equiv.) and benzyl acrylate (3a; 1.5 equiv.) in DMSO under argon and exclusion of light. The mixture was diluted with DMSO up to a total volume of $0.5 \mathrm{~mL}$, and irradiated with blue LEDs at $20-22{ }^{\circ} \mathrm{C}$. The progress of the reaction was monitored by ${ }^{1} \mathrm{H}-\mathrm{NMR}$ spectroscopy. 


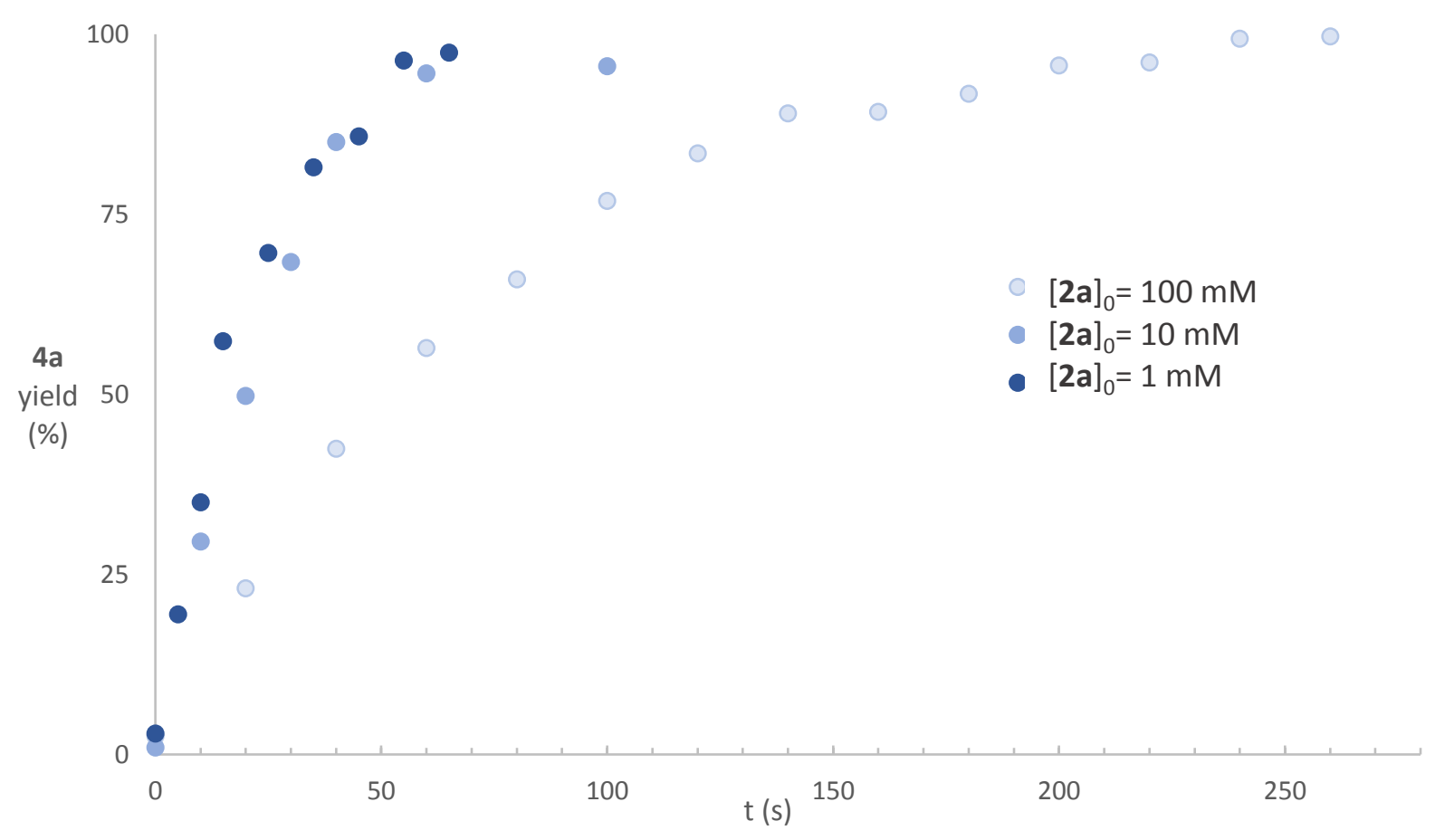

Figure S32: Reaction profile of the production of $4 a$ with different initial concentrations of $\mathbf{2 a}$ (see. Table S8) 
Table S8: Data of the formation of $\mathbf{4 a}$ with $\mathbf{3 a}$ at different initial concentrations of $\mathbf{2 a}$ (see. Figure S32).

\begin{tabular}{|c|c|c|c|c|c|c|c|}
\hline Entry & \multicolumn{2}{|c|}{$[\mathbf{2 a}]_{0}=100 \mathrm{mM}^{\mathrm{a}}$} & \multicolumn{2}{|c|}{$[\mathbf{2 a}]_{0}=10 \mathrm{mM}$} & \multicolumn{2}{|c|}{$[\mathbf{2 a}]_{0}=1 \mathrm{mM}^{\mathrm{b}}$} \\
\hline & Time $(\mathrm{s})$ & Yield 4a (\%) & Time $(\mathrm{s})$ & Yield 4a (\%) & Time (s) & Yield 4a (\%) \\
\hline 1 & 0 & 2.52 & 0 & 0 & 0 & 2.92 \\
\hline 2 & 20 & 23.07 & 10 & 29.57 & 5 & 19.43 \\
\hline 3 & 40 & 42.45 & 20 & 49.79 & 10 & 35.02 \\
\hline 4 & 60 & 56.45 & 30 & 68.399 & 15 & 57.37 \\
\hline 5 & 80 & 65.94 & 40 & 85.01 & 25 & 69.65 \\
\hline 6 & 100 & 76.84 & 60 & 94.54 & 35 & 81.52 \\
\hline 7 & 120 & 83.46 & 100 & 95.54 & 45 & 85.80 \\
\hline 8 & 140 & 89.01 & - & - & 55 & 96.37 \\
\hline 9 & 160 & 89.21 & - & - & 65 & 97.44 \\
\hline 10 & 180 & 91.73 & - & - & - & - \\
\hline 11 & 200 & 95.66 & - & - & - & - \\
\hline 12 & 220 & 96.11 & - & - & - & - \\
\hline 13 & 240 & 99.40 & - & - & - & - \\
\hline 14 & 260 & 99.72 & - & - & - & - \\
\hline
\end{tabular}

Conditions: 2a (1.0 equiv.), 10 (1.5 equiv.), and 3a (1.5 equiv.) in DMSO (0.5 mL) irradiation with blue LEDs at $20-22^{\circ} \mathrm{C}$; a) standard reaction conditions (see.

Table S4); b) DMSO- $d_{6}$ was used as solvent.

\section{Procedure for the control reaction for the inner filter effect}

A standard reaction mixture with $2 \mathrm{a}(14 \mathrm{mg}, 50 \mu \mathrm{mol}, 0.1 \mathrm{M}), 10\left(0.4 \mathrm{~mL}\right.$ in DMSO- $d_{6}, 0.2 \mathrm{M}$, $75 \mu \mathrm{mol}, 1.5$ equiv.), and 3a (11 $\mu \mathrm{L}, 75 \mu \mathrm{mol}, 1.5$ equiv.) were pre-mixed under inert atmosphere using a screw-neck vial that was wrapped in aluminum foil. All reactants were dissolved in additional DMSO- $d_{6}(0.1 \mathrm{~mL})$ under strict exclusion of light. Then $60 \mu \mathrm{L}$ of the reaction mixture was filled into a thin NMR reactor $(\mathrm{d}=1.25 \mathrm{~mm})$. The reactor was closed under inert gas, placed in an empty NMR tube, and illuminated with blue LEDs at $20-22{ }^{\circ} \mathrm{C}$. The progress of the reaction was followed by ${ }^{1} \mathrm{H}-\mathrm{NMR}$. 


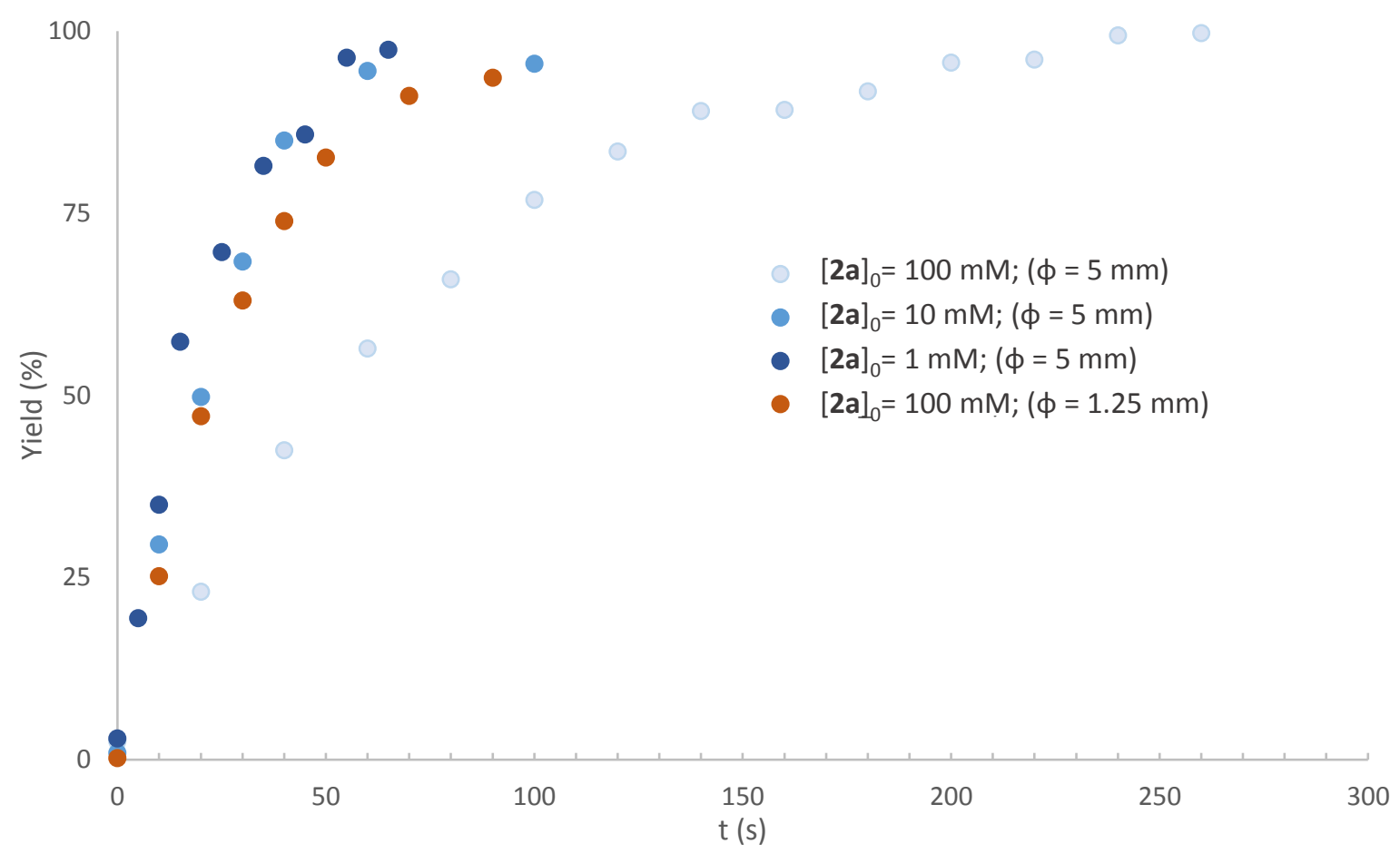

Figure S33. Inner filter effect. Overlay of the different initial concentration profiles (see. Table S8) with standard initial concentration in a thin NMR tube ( $\phi=1.25 \mathrm{~mm}$ ) (see. Table S9).

Table S9: Data for the inner filter effect experiment.

\begin{tabular}{|c|c|c|c|}
\hline Entry & Time (s) & {$[\mathbf{4 a}$ (M) } & Yield 4a (\%) \\
\hline 1 & 0 & 0.000203 & 0.20 \\
\hline 2 & 10 & 0.02519 & 25.19 \\
\hline 3 & 20 & 0.047124 & 47.12 \\
\hline 4 & 30 & 0.063005 & 63.00 \\
\hline 5 & 40 & 0.073932 & 73.93 \\
\hline 6 & 50 & 0.08265 & 82.65 \\
\hline 7 & 70 & 0.091137 & 91.14 \\
\hline 8 & 90 & 0.0936 & 93.60 \\
\hline
\end{tabular}

Conditions: $2 a(0.1 \mathrm{M})$, BuNAH (10;0.15 M) and benzyl acrylate (3a; $0.15 \mathrm{M})$ in DMSO- $d_{6}$, irradiation with blue LEDs at $20-22{ }^{\circ} \mathrm{C}$. 
In situ detection of pyridinium salt (18) by ${ }^{1} \mathrm{H}-\mathrm{NMR}$
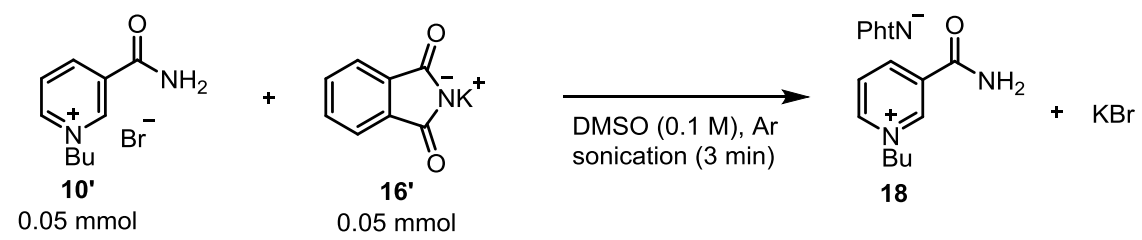

An oven-dried NMR tube was charged with 1-butyl-3-carbamoylpyridin-1-ium bromide (10'; $12.95 \mathrm{mg}, 0.05 \mathrm{mmol}$ ) and potassium phthalimide (16'; $9.24 \mathrm{mg}, 0.05 \mathrm{mmol})$. The tube was evacuated and refilled with argon (three cycles), followed by addition of anhydrous DMSO $(0.5 \mathrm{~mL})$. The mixture was then sonicated for $3 \mathrm{~min}$ and the colourless mixture turned into yellow. $\mathrm{A}^{1} \mathrm{H}-\mathrm{NMR}$ spectrum of the mixture was recorded.

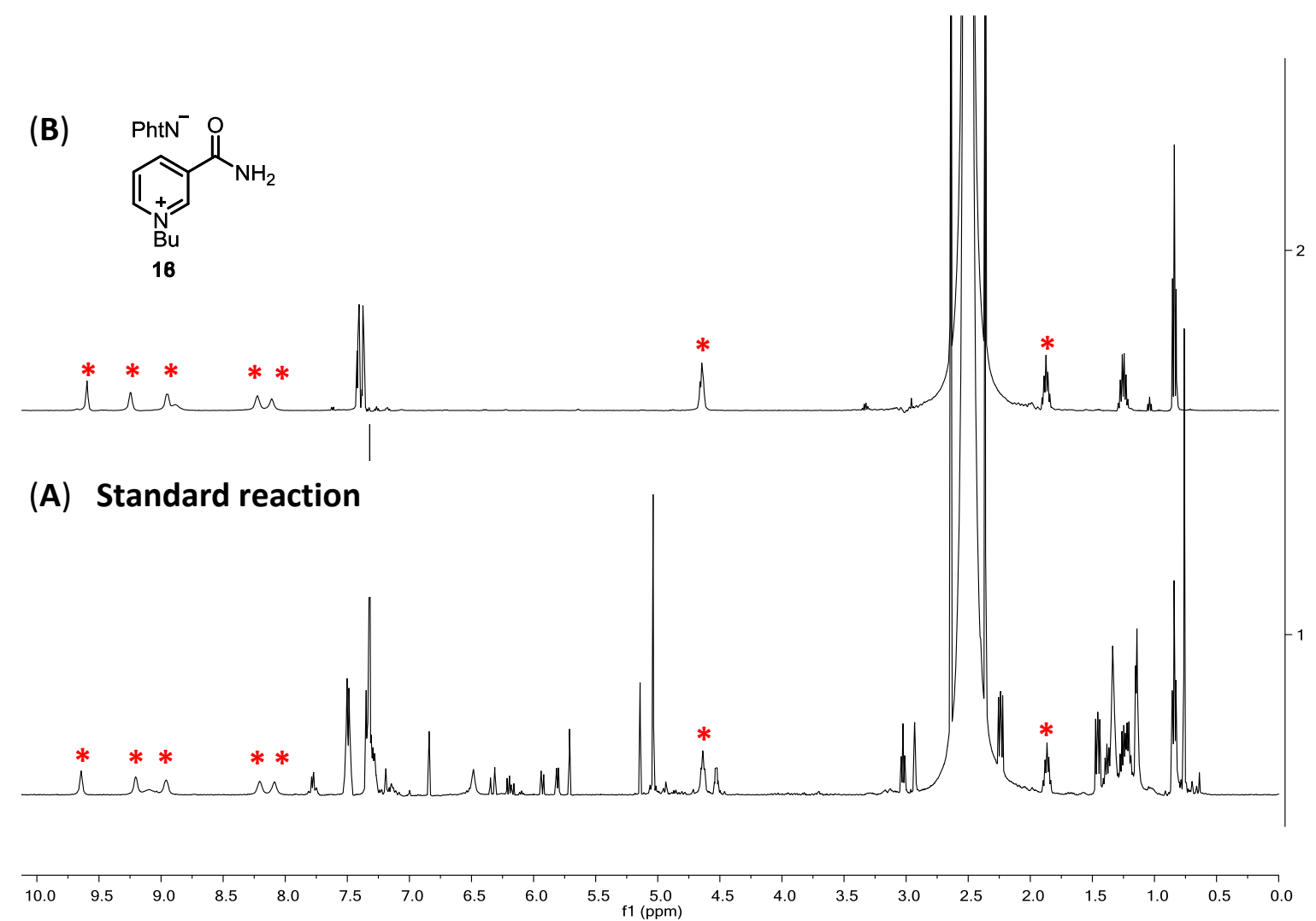

Figure S34: ${ }^{1} \mathrm{H}-\mathrm{NMR}$ of $(\mathrm{A})$ the standard reaction after complete conversion of $2 a$ (see. Figure S27); (B) 18 in DMSO; signals for 18 are highlighted in red. 


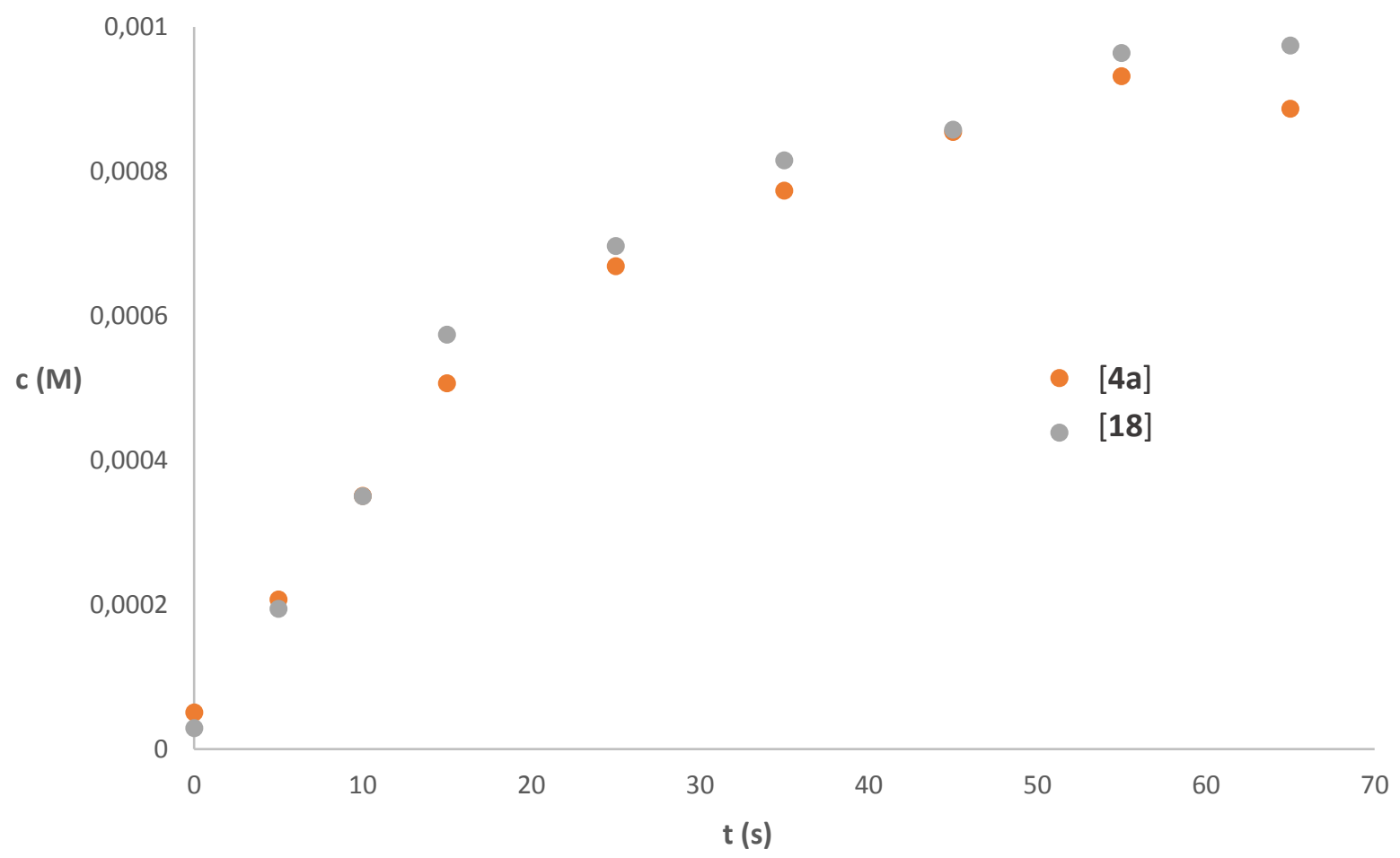

Figure S35: Formation of photo-ligated product $\mathbf{4 a}$ and pyridinium salt $\mathbf{1 8}([\mathbf{2 a}] 0=1 \mathrm{mM})$ in DMSO-d6 (see. Table S10).

Table S10: Formation of $\mathbf{4 a}$ and pyridinium salt $\mathbf{1 8}\left([\mathbf{2 a}]_{0}=1 \mathrm{mM}\right)$. (see. Figure S35)

\begin{tabular}{|c|c|c|c|}
\hline Entry & Time $(\mathrm{s})$ & {$[\mathbf{4 a}](\mathrm{mM})$} & {$[\mathbf{1 8}](\mathrm{mM})$} \\
\hline 1 & 0 & 0 & 0 \\
\hline 2 & 20 & 0.136 & 0.139 \\
\hline 3 & 40 & 0.274 & 0.275 \\
\hline 4 & 60 & 0.396 & 0.406 \\
\hline 5 & 80 & 0.594 & 0.625 \\
\hline 6 & 100 & 0.707 & 0.748 \\
\hline 7 & 120 & 0.784 & 0.818 \\
\hline 8 & 140 & 0.824 & 0.879 \\
\hline 9 & 160 & 0.854 & 0.912 \\
\hline
\end{tabular}

Reaction conditions: $\mathbf{2 a}(50 \mu \mathrm{mol}), 3$ a (1.5 equiv.), 10 (1.5 equiv.) in DMSO- $d_{6}$ irradiation with blue LEDs at $20-22{ }^{\circ} \mathrm{C}$. 


\section{Preliminary evaluation of the photo-coupling with NADH (11)}

\section{Profiling of photo-ligation using NADH (11) as reductant by ${ }^{1} H-N M R$}
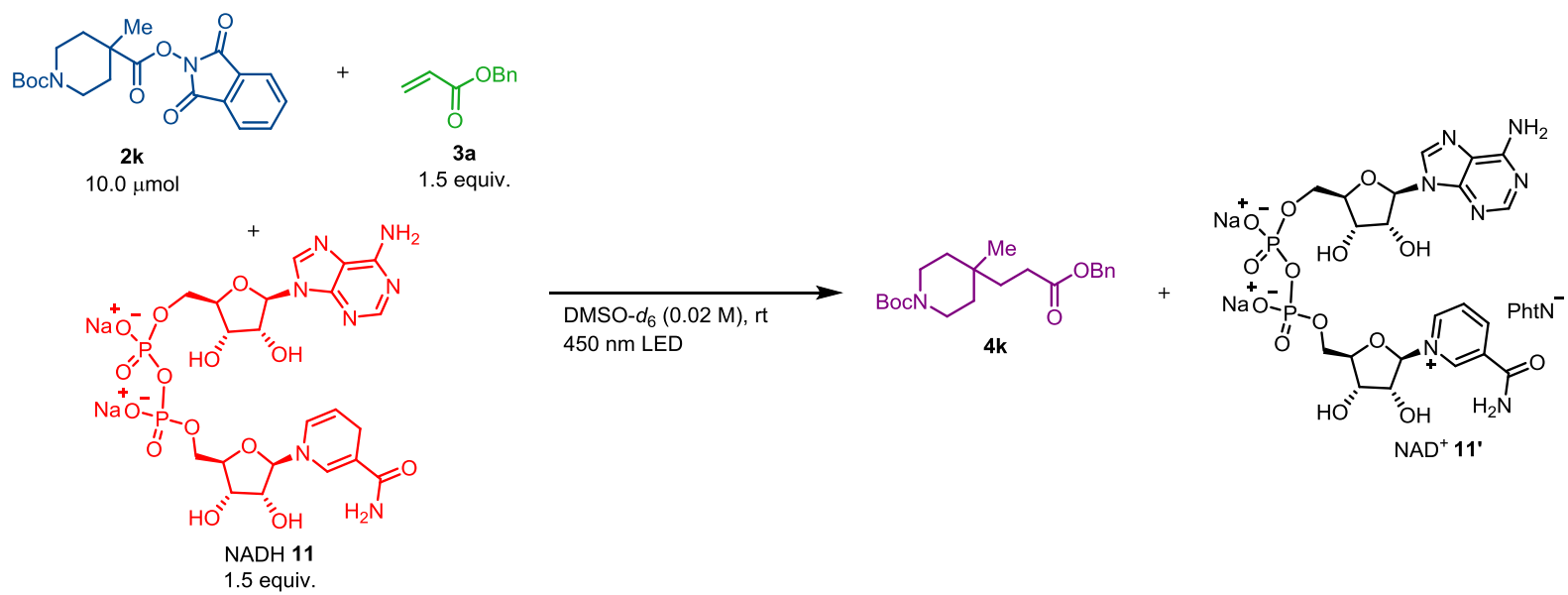

The reaction was performed under strict exclusion of light. Redox-active ester $\mathbf{2 k}$ (3.9 mg, 10.0 $\mu \mathrm{mol}, 1.0$ equiv.) and $\mathrm{NADH}(11 ; 10.6 \mathrm{mg}, 15.0 \mu \mathrm{mol}, 1.5$ equiv.) were weighed carefully in a NMR tube. After closing with a rubber septum, the vial was evacuated, refilled with argon (three times) and dry degassed DMSO- $d_{6}(0.5 \mathrm{~mL})$ containing benzyl acrylate (3a; $2.4 \mathrm{mg}, 15$ $\mu \mathrm{mol}, 1.5$ equiv.) was added. The mixture was then irradiated with $450 \mathrm{~nm}$ LED at $20-22^{\circ} \mathrm{C}$. The progress of the reaction was monitored by ${ }^{1} \mathrm{H}-\mathrm{NMR}$.

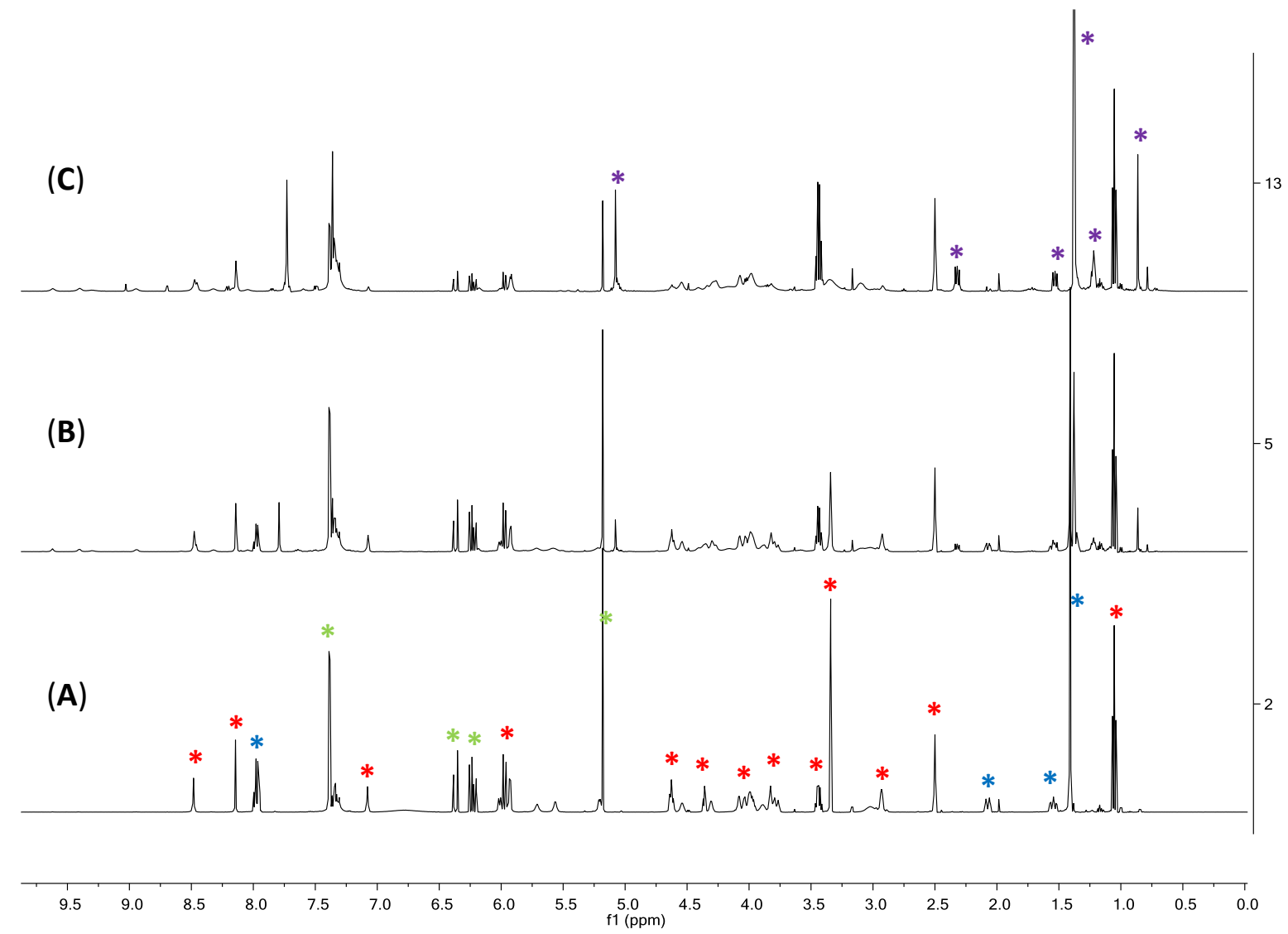

Figure S36: ${ }^{1} \mathrm{H}-N M R$ spectra of the conversion of $\mathbf{2} \boldsymbol{k}$ to $\mathbf{4} \boldsymbol{k}$ using $N A D H(11)$ as reductant after (A) $0 \mathrm{~min}$; (B) $6 \mathrm{~min}$; (C) 73 min of irradiation. 


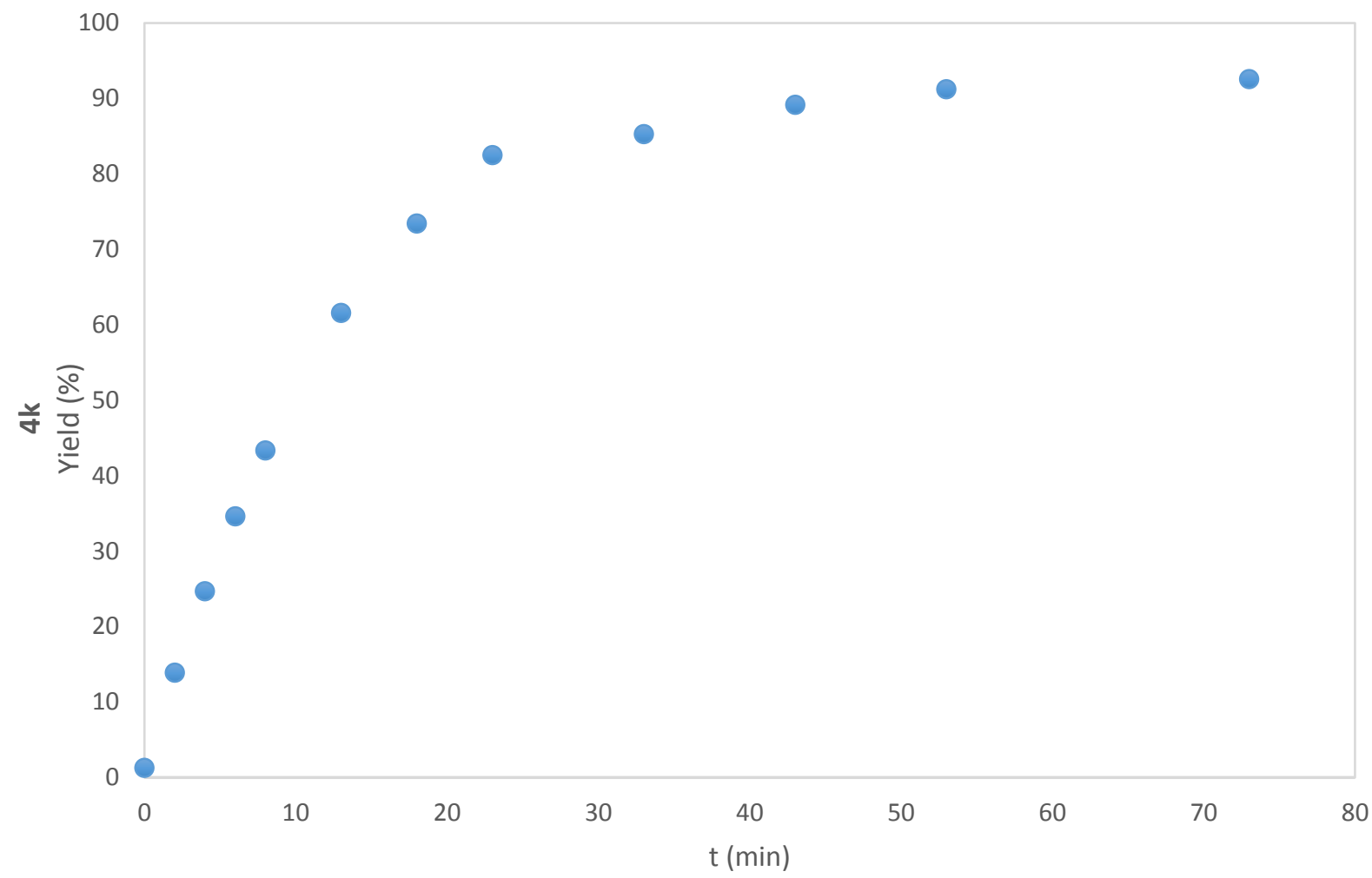

Figure S37: Progress of photo-ligation reaction using NADH (11) as reductant monitored by ${ }^{1} \mathrm{H}-\mathrm{NMR}$ (see. Table S11).

Table S11: Data of photo-ligation promoted by NADH (11) (see. Figure S37).

\begin{tabular}{|c|c|c|c|}
\hline Entry & Time (min) & {$[\mathbf{4 k}(\mathbf{M})$} & Yield $\mathbf{4 k} \mathbf{( \% )}$ \\
\hline 1 & 0 & 0.0002 & 1 \\
\hline 2 & 2 & 0.002 & 13 \\
\hline 3 & 4 & 0.004 & 24 \\
\hline 4 & 6 & 0.006 & 34 \\
\hline 5 & 8 & 0.008 & 43 \\
\hline 6 & 13 & 0.012 & 61 \\
\hline 7 & 18 & 0.014 & 73 \\
\hline 8 & 23 & 0.016 & 82 \\
\hline 9 & 33 & 0.017 & 85 \\
\hline 10 & 43 & 0.017 & 89 \\
\hline 11 & 53 & 0.018 & 91 \\
\hline 12 & 73 & 0.018 & 92 \\
\hline
\end{tabular}




\section{Mechanistic studies}

\section{Radical clock experiments}

Syntheses of benzyl 4-cyclopentylbutanoate (4ab) and benzyl non-8-enoate (4ab')

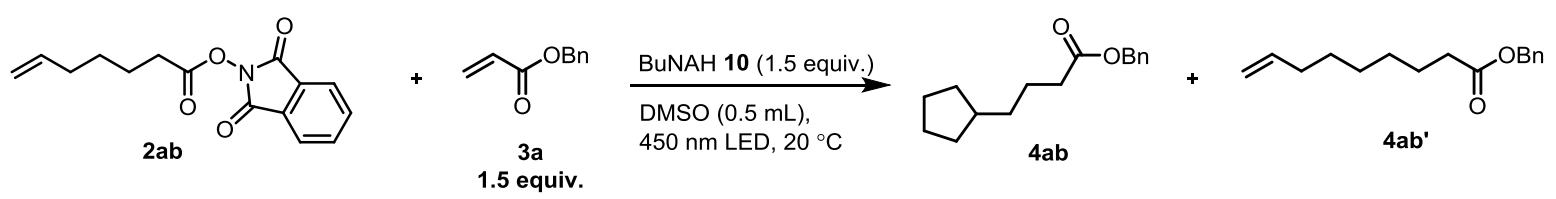

Following General procedure B3, 1,3-dioxoisoindolin-2-yl hept-6-enoate (2ab, 1.0 equiv.), benzyl acrylate (3a, 1.5 equiv.) and 1-butyl-1,4-dihydropyridine-3-carboxamide (10, 1.5 equiv.) in DMSO were illuminated for $5 \mathrm{~min}$. The product was obtained as a mixture of $4 \mathrm{ab}$ and $4 \mathbf{a b}^{\prime}$. The yield was determined by ${ }^{1} \mathrm{H}-\mathrm{NMR}$ using 1,1,2,2-tetrachloroethane as internal standard.

Table S12: Data of radical clock experiment.

\begin{tabular}{|c|c|c|c|c|}
\hline Entry & {$[\mathbf{2} \mathbf{a b}]_{0}(\mathbf{M})$} & Conversion 2ab (\%) & Ratio (4ab/4ab') & Yield (4ab+4ab') (\%) \\
\hline $1^{\mathrm{b}}$ & 0.01 & $>99$ & $94: 6$ & 78 \\
\hline $2^{\mathrm{b}}$ & 0.05 & $>99$ & $80: 20$ & 66 \\
\hline $3^{\mathrm{c}}$ & 0.1 & $>99$ & $51: 49$ & 96 \\
\hline $4^{\mathrm{c}}$ & 0.2 & $>99$ & $40: 60$ & 82 \\
\hline
\end{tabular}

a) The yield was determined by ${ }^{1} \mathrm{H}-\mathrm{NMR}$ with 1,1,2,2-tetrachloroethane as an internal standard; b) $50 \mu \mathrm{mol}$ of $2 \mathrm{ab}$; c) $100 \mu \mathrm{mol}$ of $2 \mathrm{ab}$.

Characterization of benzyl 4-cyclopentylbutanoate (4ab)

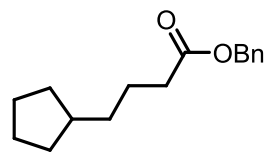

Appearance: colorless oil.

TLC: $R_{f}=0.38$ (petroleum ether/EtOAc $=10: 1$, UV-active and stains in permanganate).

${ }^{1} \mathrm{H}-\mathrm{NMR}\left(400 \mathrm{MHz}, \mathrm{CDCl}_{3}\right) \delta(\mathrm{ppm})=7.42-7.29(\mathrm{~m}, 5 \mathrm{H}), 5.12(\mathrm{~s}, 2 \mathrm{H}), 2.35(\mathrm{t}, J=7.5 \mathrm{~Hz}, 2 \mathrm{H})$, $1.79-1.70(\mathrm{~m}, 3 \mathrm{H}), 1.70-1.61(\mathrm{~m}, 2 \mathrm{H}), 1.61-1.54(\mathrm{~m}, 2 \mathrm{H}), 1.52-1.44(\mathrm{~m}, 2 \mathrm{H}), 1.35-1.25$ $(\mathrm{m}, 2 \mathrm{H}), 1.13-0.98(\mathrm{~m}, 2 \mathrm{H})$.

${ }^{13} \mathrm{C}-\mathrm{NMR}\left(101 \mathrm{MHz}, \mathrm{CDCl}_{3}\right) \delta(\mathrm{ppm})=173.9,136.3,128.7,128.3,128.3,66.2,40.0,35.8,34.7$, 32.7, 25.3, 24.3.

HRMS (ESI-TOF) calc'd for $\mathrm{C}_{16} \mathrm{H}_{22} \mathrm{O}_{2} \mathrm{Na}$ [M+Na] ${ }^{+}: 269.1512$; found: 269.1517.

All the data are in accordance with the literature. ${ }^{7}$ 
Characterization of benzyl non-8-enoate (4ab')<smiles>C=CCCCCCCC(=O)OCCCCCCC</smiles>

Appearance: colorless oil.

TLC: $R_{f}=0.36$ (petrol ether/EtOAc $=10: 1$, UV-active and stains blue in vanillin).

${ }^{1} \mathrm{H}-\mathrm{NMR}\left(400 \mathrm{MHz}, \mathrm{CDCl}_{3}\right) \delta(\mathrm{ppm})=7.44-7.30(\mathrm{~m}, 5 \mathrm{H}), 5.79(\mathrm{ddt}, J=16.9,10.2,6.6 \mathrm{~Hz}, 1 \mathrm{H})$, $5.11(\mathrm{~s}, 2 \mathrm{H}), 5.07-4.82(\mathrm{~m}, 2 \mathrm{H}), 2.35(\mathrm{t}, \mathrm{J}=7.5 \mathrm{~Hz}, 2 \mathrm{H}), 2.08-1.94(\mathrm{~m}, 2 \mathrm{H}), 1.75-1.57(\mathrm{~m}$, $2 \mathrm{H}), 1.43-1.27(\mathrm{~m}, 6 \mathrm{H})$.

${ }^{13} \mathrm{C}-\mathrm{NMR}\left(101 \mathrm{MHz}, \mathrm{CDCl}_{3}\right) \delta(\mathrm{ppm})=173.7,139.0,136.1,128.5,128.2,128.2,114.3,66.1$, 34.3, 33.7, 29.0, 28.7, 28.7, 24.9.

HRMS (ESI-TOF) calc'd for $\mathrm{C}_{16} \mathrm{H}_{22} \mathrm{O}_{2} \mathrm{Na}$ [M+Na] $]^{+}: 269.1512$; found: 269.1517.

\section{Deuterium labelling experiment}

Synthesis of 1-Butyl-4,4-dideuterio-1,4-dihydronicotinamide (10- $\left.d_{2}\right)$

1-Butyl-4,4-dideuterio-1,4-dihydronicotinamide (10- $\left.d_{2}\right)$ was prepared from 10 ' by sequential reduction and oxidation following literature known procedure. ${ }^{14}$ The product was obtained after four reduction and oxidation cycles with 94\% D-incorporation as a yellow oil.

The spectroscopic data was found consistent with the literature. ${ }^{14}$

Synthesis of benzyl 3-(1-methylcyclohexyl)propanoate-d $d_{1}\left(\mathbf{4 a - d _ { 1 } )}\right.$

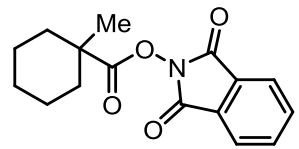

$2 \mathrm{a}$
$.15 \mathrm{mmol}$

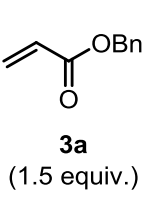

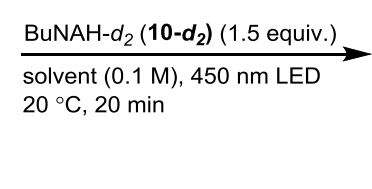

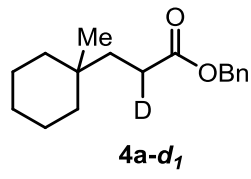

$4 \mathrm{a}-d_{1}$

General procedure B3 was applied using redox-active ester $2 \mathrm{a}(43.1 \mathrm{mg}, 0.15 \mathrm{mmol}, 1.0$ equiv.), BuNAH- $d_{2}\left(10-d_{2} ; 41.0 \mathrm{mg}, 0.26 \mathrm{mmol}, 1.5\right.$ equiv., $94 \% \mathrm{D}$; determined by $\left.{ }^{1} \mathrm{H}-\mathrm{NMR}\right)$, and benzyl acrylate (3a; $36.5 \mathrm{mg}, 0.26 \mathrm{mmol}, 1.5$ equiv.) under illumination with blue LEDs at $20{ }^{\circ} \mathrm{C}$ for $20 \mathrm{~min}$ in the appropriate solvent $(0.1 \mathrm{M})$ to yield $4 \mathrm{a}-\mathrm{d}_{1}$. Yields and $\mathrm{D} \%$ were determined by ${ }^{1} \mathrm{H}-\mathrm{NMR}$ using 1,1,2,2-tetrachloroethane as internal standard.

\begin{tabular}{|c|c|c|c|}
\hline Entry & Solvent & Yield $4 a-d_{1}(\%)$ & Ratio $\left(4 a / 4 a-d_{1}\right)$ \\
\hline 1 & DMSO- $d_{6}$ & 57 & $31: 69$ \\
\hline 2 & DMSO & 50 & $32: 68$ \\
\hline 3 & $\mathrm{DMSO} / \mathrm{H}_{2} \mathrm{O}$ 1:1 & $35^{a}$ & $30: 70$ \\
\hline
\end{tabular}

a) $85 \%$ conversion after 30 min illumination.

Appearance: colorless oil.

TLC: $R f=0.22$ (pentane/DCM = 2:1, UV-active stains in PMA).

${ }^{1} \mathrm{H}-\mathrm{NMR}\left(400 \mathrm{MHz}, \mathrm{CDCl}_{3}\right) \delta(\mathrm{ppm})=7.41-7.29(\mathrm{~m}, 5 \mathrm{H}), 5.11(\mathrm{~s}, 2 \mathrm{H}), 2.35-2.26(\mathrm{~m}, 1.2 \mathrm{H})$, $1.65-1.56(\mathrm{~m}, 2 \mathrm{H}), 1.49-1.37(\mathrm{~m}, 5 \mathrm{H}), 1.36-1.26(\mathrm{~m}, 1 \mathrm{H}), 1.30-1.17(\mathrm{~m}, 4 \mathrm{H}), 0.85(\mathrm{~s}, 3 \mathrm{H})$. 
${ }^{13} \mathrm{C}-\mathrm{NMR}\left(126 \mathrm{MHz}, \mathrm{CDCl}_{3}\right) \delta(\mathrm{ppm})=174.6,136.3,128.7,128.4,128.3,66.3,37.6,36.8,32.5$, 29.1, 29.0, 28.9, 28.7, 26.5, 24.6, 22.1.

HRMS (ESI-TOF) calc'd for $\mathrm{C}_{17} \mathrm{H}_{23} \mathrm{DO}_{2} \mathrm{Na}[\mathrm{M}+\mathrm{Na}]^{+}: 284.1731$, found: 284.1730 .

\section{Determination of the quantum yield of the photo-ligation}

The photon flux of the spectrophotometer was determined by standard potassium ferrioxalate actinometry. ${ }^{15}$

Preparation of buffered phenanthroline solution (Solution A)

A buffered solution was made by dissolving phenanthroline $(25 \mathrm{mg}, 138.0 \mu \mathrm{mol})$ and anhydrous sodium acetate $(5.63 \mathrm{~g}, 0.07 \mathrm{~mol})$ in $0.5 \mathrm{M} \mathrm{H}_{2} \mathrm{SO}_{4}(25 \mathrm{~mL})$.

Preparation of potassium ferrioxalate solution (Solution B)

The solution of potassium ferrioxalate trihydrate in $0.05 \mathrm{M} \mathrm{H}_{2} \mathrm{SO}_{4}$ was prepared in a dark laboratory illuminated with red light. Potassium ferrioxalate trihydrate (736 mg, $1.5 \mathrm{mmol}$ ) was dissolved in $0.05 \mathrm{M} \mathrm{H}_{2} \mathrm{SO}_{4}(10 \mathrm{~mL})$ in a volumetric flask to yield a $0.15 \mathrm{M}$ solution.

Four cuvettes were charged with solution $B(2 \mathrm{~mL})$. The cuvettes were irradiated at $\lambda=450$ $\mathrm{nm}$ with an excitation slit width of $5.0 \mathrm{~nm}$ for the time as indicated in table S1. After irradiation, phenanthroline solution $(0.35 \mathrm{~mL}$ solution A) was added to every cuvette (irradiated and non-irradiated). The solutions were then allowed to rest for $1 \mathrm{~h}$ in the dark to complete the chelation of ferrous ions to the phenanthroline. After $1 \mathrm{~h}$, absorbance was measured for all four solutions (and a $0.05 \mathrm{M} \mathrm{H}_{2} \mathrm{SO}_{4}$ blank) solution at $\lambda=510 \mathrm{~nm}$.

For the calculation of photon flux the following equations were used:

$$
\begin{gathered}
\text { mol of } F e^{2+}=\frac{V * \Delta \mathrm{A}}{\mathrm{l} * \mathcal{E}} \\
\text { Photon flux }=\frac{\text { mol of } F e^{2+}}{\Phi * \mathrm{t} * \mathrm{f}}
\end{gathered}
$$

Where $V$ is the total volume of the solution $(2.35 \mathrm{~mL}), \Delta \mathrm{A}$ is the difference in absorption between the irradiated and non-irradiated samples, $I$ is the path length $(1 \mathrm{~cm})$, and $\varepsilon$ is the molar absorptivity at $\lambda=510 \mathrm{~nm}\left(11110 \mathrm{~L} \mathrm{~mol}^{-1} \mathrm{~cm}^{-1}\right),{ }^{15} \phi$ is the quantum yield for the potassium ferrioxalate actinometer (1.01), ${ }^{15} \mathrm{t}$ is the time of irradiation (s), and $\mathrm{f}$ is the fraction of light absorbed at $\lambda=450 \mathrm{~nm}$ by potassium ferrioxalate $(0.99833) .{ }^{15}$ 
Determination of photon flux

Table S13: Determination of photon flux using ferrioxalate actinometry

\begin{tabular}{|c|c|c|c|c|c|c|}
\hline & $\begin{array}{c}\text { Irradiation } \\
\text { time }(\mathrm{s})\end{array}$ & $\mathrm{Abs}(\mathrm{A})$ & $\Delta \mathrm{A}$ & $\mathrm{V}\left(\mathrm{Lx} 10^{-3}\right)$ & $\mathrm{Fe}^{2+}\left(\mathrm{molx10} 0^{-8}\right)$ & $\begin{array}{c}\text { Photon flux } \\
\left.\text { (einstein. }{ }^{-1} \times 10^{-9}\right)\end{array}$ \\
\hline 1 & 0 & 0.40 & 0 & 2.35 & - & - \\
\hline 2 & 11.40 & 0.69 & 0.29 & 2.35 & 6.08 & 5.28 \\
\hline 3 & 20.90 & 0.81 & 0.41 & 2.35 & 8.72 & 4.14 \\
\hline 4 & 30.90 & 1.02 & 0.61 & 2.35 & 12.99 & 4.17 \\
\hline & & & & & Average: 4.53 \\
\hline & & & & & Std. dev.: 0.65 \\
\hline
\end{tabular}

Considering $95 \%$ confidence interval: average photon flux of the spectrofluorometer is (4.53 $\pm 0.74) \times 10^{-9}$ einstein. $s^{-1}$.

Determination of the quantum yield

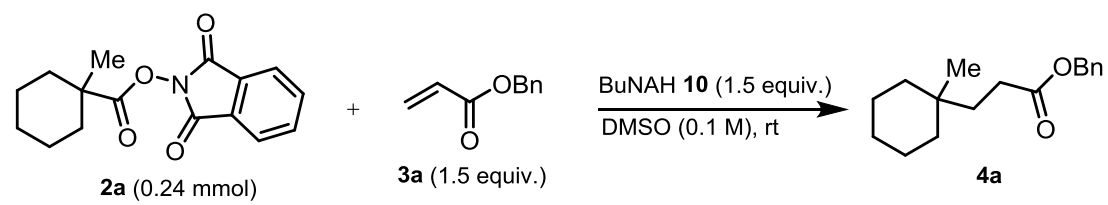

The reactions were set up inside the glove box. A cuvette was charged with redox-active ester 2a (68.9 mg, $0.24 \mathrm{mmol}, 1.0$ equiv.) and BuNAH (10; $64.9 \mathrm{mg}, 0.36,1.5$ equiv.). Anhydrous DMSO $(2.4 \mathrm{~mL})$ was added to the mixture, followed by the addition of benzyl acrylate (3a; $58.4 \mathrm{mg}, 0.36 \mathrm{mmol}, 1.5$ equiv.). The cuvette was wrapped in an aluminium foil and was brought outside of the glove box. The absorption was measured at $\lambda=450 \mathrm{~nm}$. The sample was irradiated in the spectrofluorometer for the indicated period of time (table S2) at $\lambda=450$ $\mathrm{nm}$ with an excitation slit width of $5.0 \mathrm{~nm}$. Internal standard, 1,3,5-trimethoxybenzene (40.36 $\mathrm{mg}, 0.24 \mathrm{mmol}$ ) was added to the cuvette. The mixture was shaken and $0.5 \mathrm{~mL}$ aliquot was used to measure the NMR yield by using no- ${ }^{1} \mathrm{H}-\mathrm{NMR}$.

$$
\text { Quantum yield, } \Phi=\frac{\text { mol of } \mathbf{4 a}}{\text { photon flux } * \mathrm{t} * \mathrm{f}}
$$

Where $t$ is the time of the reaction and $f$ is the fraction of the light absorbed at $450 \mathrm{~nm}$ by the reaction mixture $=1-10^{-A}$. Absorbance $(A)$ of the reaction mixture at $\lambda=450 \mathrm{~nm}$ is 1.99 . So, $f$ $=1-10^{-1.99}=0.9897$.

Additionally, the absorbance (A) of the reaction mixture after 90 min irradiation at $\lambda=450 \mathrm{~nm}$ was controlled. The corresponding fraction of the light absorbed was determined to be $f=1-$ $10^{-2.79}=0.9984$, which is approximately equal to the starting value. 
Table S14: Determination of the quantum yield of the photo-ligation reaction

\begin{tabular}{|c|c|c|c|c|c|}
\hline & Irradiation time $(s)$ & $\begin{array}{c}\text { NMR yield } \mathbf{4 a} \\
(\%)\end{array}$ & $\mathbf{4 a ( m o l x 1 0 ^ { - 5 } )}$ & Quantum yield $(\phi)$ & Error $(\Delta \phi)$ \\
\hline 1 & 3720 & 21 & 5.04 & 3.02 & 0.87 \\
\hline 2 & 3720 & 19.5 & 4.68 & 2.80 & 0.85 \\
\hline 3 & 4500 & 24 & 5.76 & 2.85 & 0.75 \\
\hline
\end{tabular}

Considering $5 \%$ error in measuring the NMR yield, the error in determining the mol of $4 \mathrm{a}$ is $1.20 \times 10^{-5}$.

The following equations were used to calculate the error in determining the quantum yield.

$$
\Phi=\frac{\text { mol of } 4 a}{\text { photon flux } * t * f}
$$

The error in measuring the quantum yield $(\phi)$ is

$$
\Delta \Phi=\sqrt{\left[\frac{\delta \Phi}{\delta(\text { mol of } 4 a)} * \Delta(\text { mol of } 4 a)\right]^{2}+\left[\frac{\delta \Phi}{\delta(\text { photon flux })} * \Delta(\text { photon flux })\right]^{2}}
$$

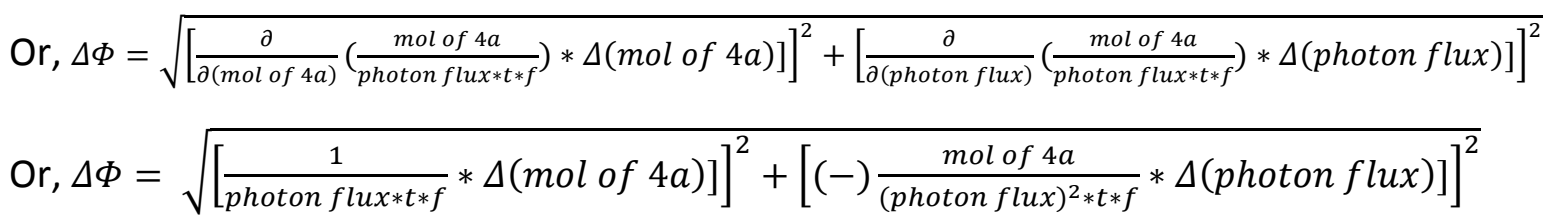

$$
\text { Or, } \Delta \Phi=\sqrt{\left.\left.\left[\frac{1}{\text { photon flux } * t * f} * \Delta(\text { mol of } 4 a)\right]\right]^{2}+\left[\frac{\text { mol of } 4 a}{(\text { photon } f l u x)^{2} * t * f} * \Delta(\text { photon flux })\right]\right]^{2}}
$$

$$
\text { Thus, } \Delta \Phi_{1}=\sqrt{\left[\frac{1.20 * 10^{-6}}{4.53 * 10^{-9} * 3720 * 0.9984}\right]^{2}+\left[\frac{5.04 * 10^{-5} * 7.38 * 10^{-10}}{\left(4.53 * 10^{-9}\right)^{2} * 3720 * 0.9984}\right]^{2}}=0.871
$$

$$
\begin{aligned}
& \Delta \Phi_{2}=\sqrt{\left[\frac{1.20 * 10^{-6}}{4.53 * 10^{-9} * 3720 * 0.9984}\right]^{2}+\left[\frac{4.68 * 10^{-5} * 7.38 * 10^{-10}}{\left(4.53 * 10^{-9}\right)^{2} * 3720 * 0.9984}\right]^{2}}=0.852 \\
& \Delta \Phi_{3}=\sqrt{\left[\frac{1.20 * 10^{-6}}{4.53 * 10^{-9} * 4500 * 0.9984}\right]^{2}+\left[\frac{5.76 * 10^{-5} * 7.38 * 10^{-10}}{\left(4.53 * 10^{-9}\right)^{2} * 4500 * 0.9984}\right]^{2}}=0.754
\end{aligned}
$$

Average quantum yield of the photo-ligation reaction was calculated to be

$$
\bar{\Phi}=\frac{\Phi_{1}+\Phi_{2}+\Phi_{3}}{3}=2.89 .
$$

The error in measuring the average quantum yield of the photo-ligation reaction is

$$
\begin{gathered}
\Delta \bar{\Phi}=\sqrt{\left(\frac{\delta \bar{\Phi}}{\delta \Phi_{1}} * \Delta \Phi_{1}\right)^{2}+\left(\frac{\delta \bar{\Phi}}{\delta \Phi_{2}} * \Delta \Phi_{2}\right)^{2}+\left(\frac{\delta \bar{\Phi}}{\delta \Phi_{3}} * \Delta \Phi_{3}\right)^{2}} \\
\text { Or, } \Delta \bar{\Phi}=\sqrt{\left(\frac{1}{3} * \Delta \Phi_{1}\right)^{2}+\left(\frac{1}{3} * \Delta \Phi_{2}\right)^{2}+\left(\frac{1}{3} * \Delta \Phi_{3}\right)^{2}}=0.48
\end{gathered}
$$


After taking into account all these errors, the average quantum yield of the photo-ligation reaction, $\bar{\Phi}=2.9 \pm 0.5$.

\section{UV-Vis study}

UV-Vis absorption spectra of the BuNAH (10), redox-active ester $\mathbf{2 a}$, and the mixture of $\mathbf{1 0}$ and $2 \mathrm{a}$ at different concentration were measured to provide information on the formation of EDA complex. From the study it is evident that there is no such difference in absorption on addition of $\mathbf{2 a}$, which defies the formation of EDA complex (see Figure S38). An EDA complex may be formed but absorption enhancement is only marginal at the wavelength of irradiation $(450 \mathrm{~nm})$ (see Figure S40).

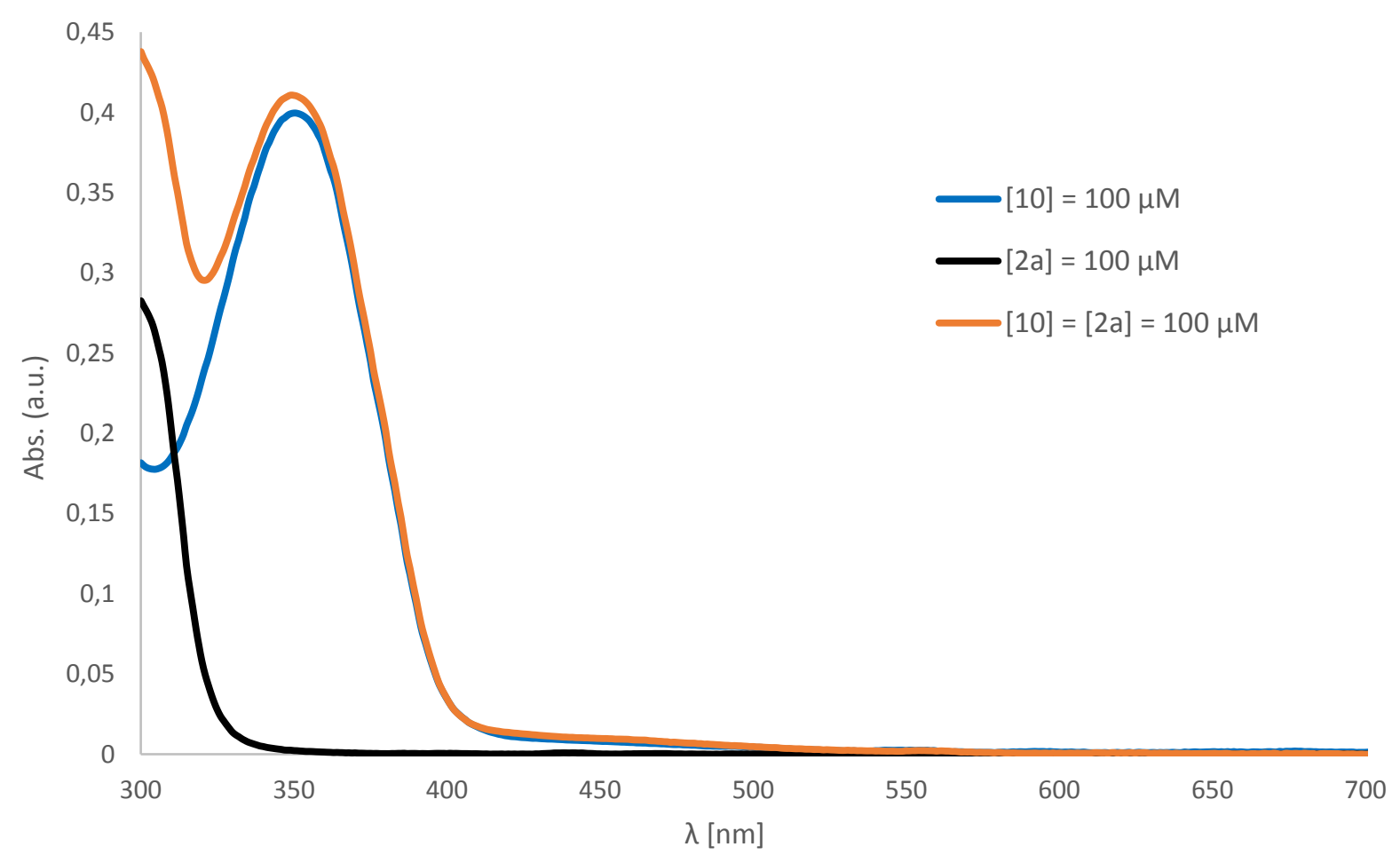

Figure S38: UV-Vis spectra of $2 a, 10$ and their 1:1 mixture at 100 uM concentration. Complete spectrum cannot be acquired due to detector saturation. 


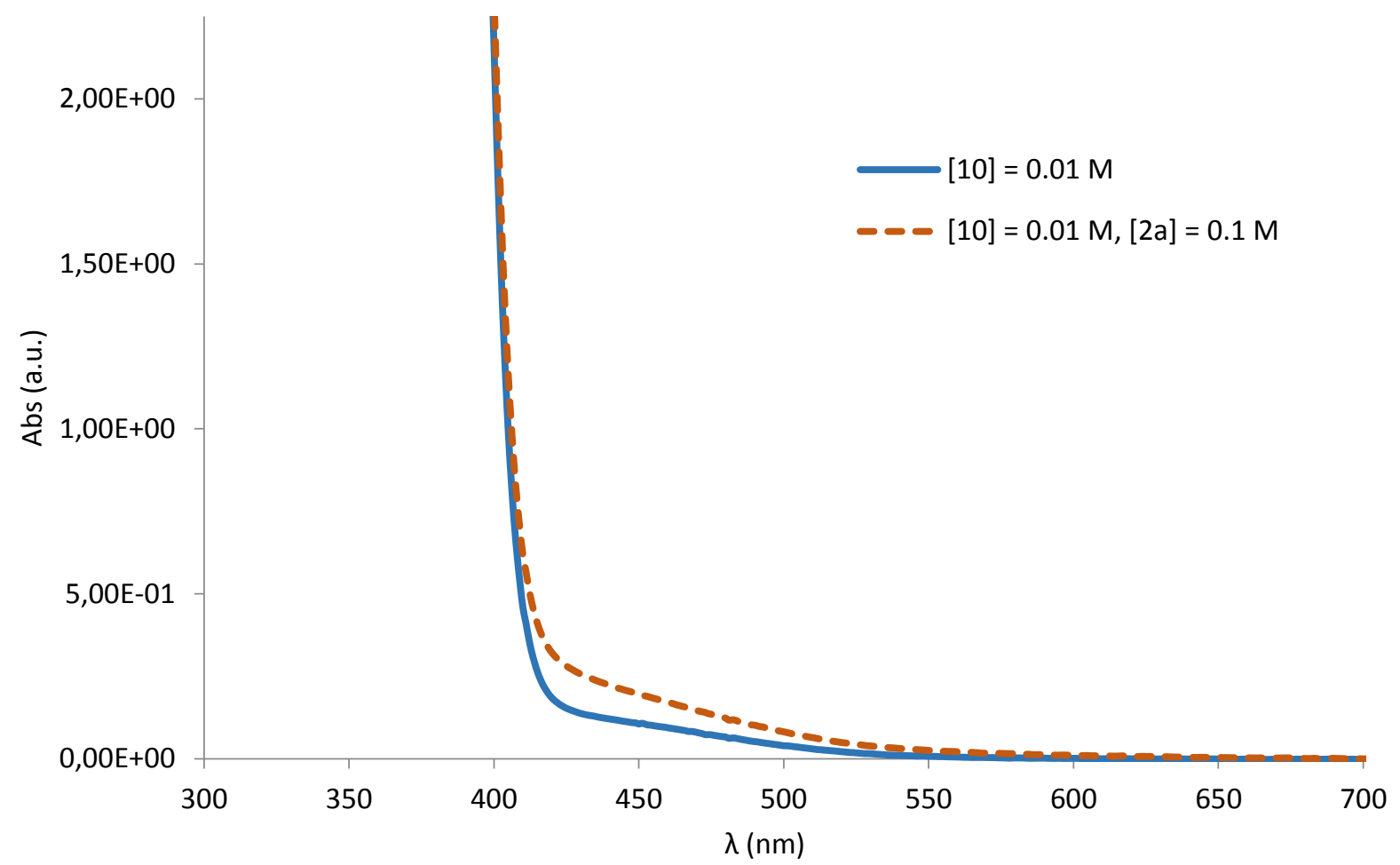

Figure S39: Absorbance of BuNAH (10) before and after quenching with 10 equiv. of redoxactive ester $\mathbf{2 a}$.

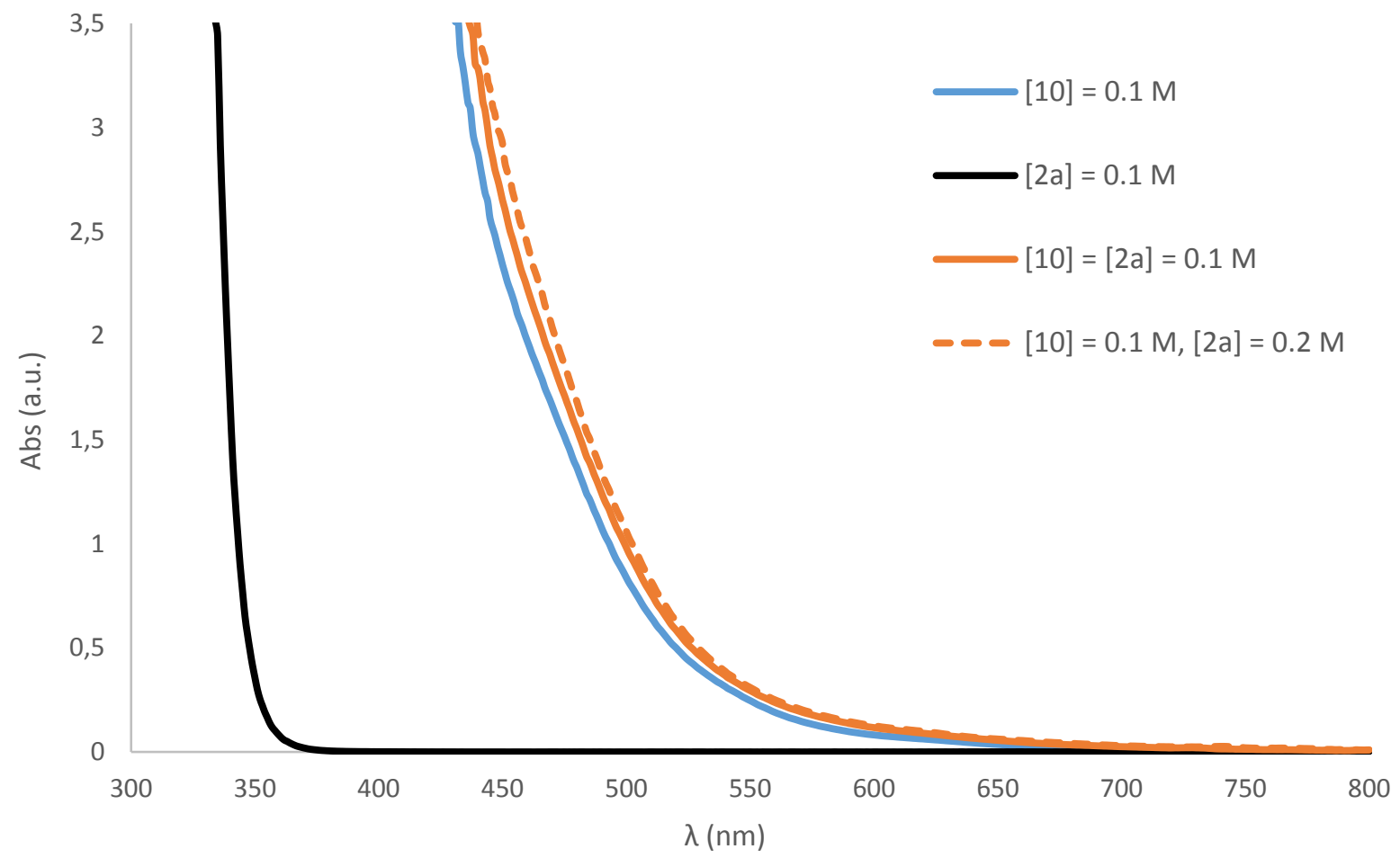

Figure S40: UV-Vis spectra of $\mathbf{2 a}, \mathbf{1 0}$ and their $1: 1$ mixture at $0.1 \mathrm{M}$ (standard preparative reaction concentration). Marginal enhancement of absorption may indicate the presence of an EDA complex. The spectrometer data may be affected by the high concentration of the sample. 


\section{Steady-state fluorescence}

Emission spectrum of BuNAH (10) and BuNAH (10) - redox-active ester 2 a mixture

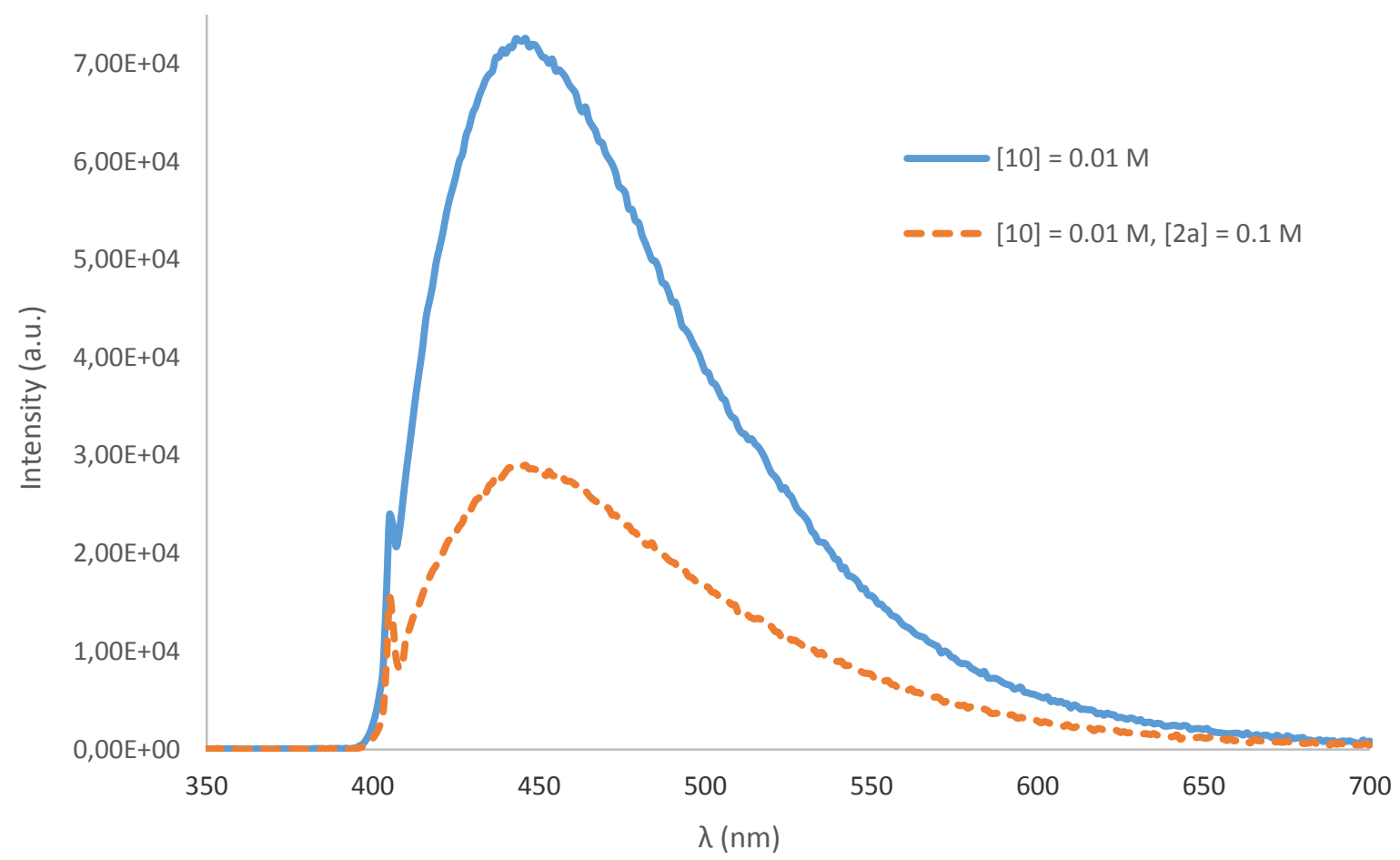

Figure S41: Emission spectra of BuNAH (10) with excitation at $405 \mathrm{~nm}$ in absence or presence of redox-active ester $\mathbf{2 a}$. 
Excitation spectrum of BUNAH (10) and BuNAH (10) - redox-active ester $2 a$ mixture

Excitation spectra was recorded to confirm that no modifications on the excitation profile of the sample were observed in the range of concentrations used in the quenching experiments.

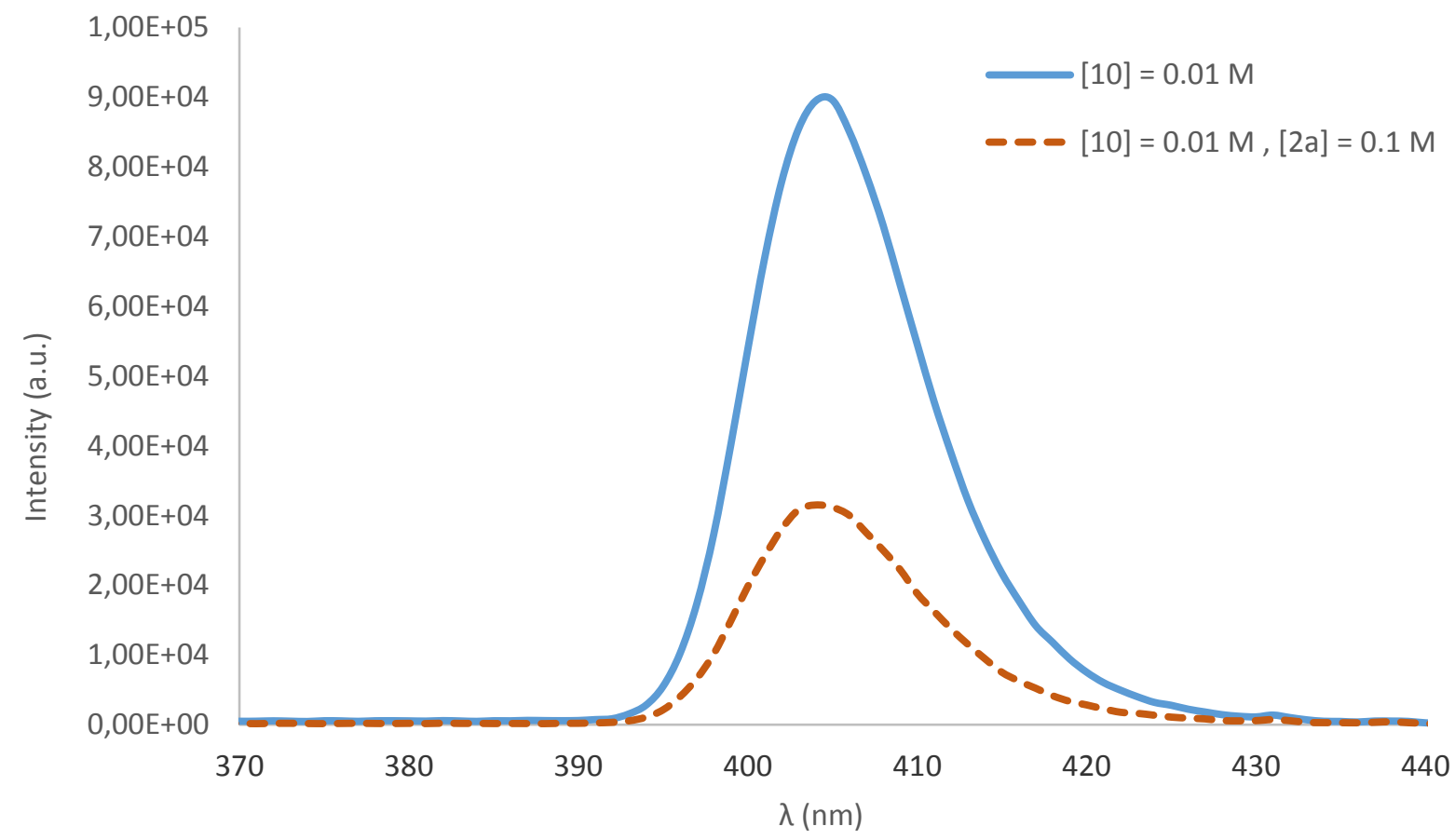

Figure S42: Excitation scan of BuNAH (10) before and after quenching with 10 equiv. of redoxactive ester $\mathbf{2} \boldsymbol{a}$. The excitation profile does not reveal a new species upon mixing $\mathbf{1 0}$ and $\mathbf{2 a}$. 
Stern-Volmer quenching experiment

Emission scans were measured at an excitation wavelength, $\lambda=405 \mathrm{~nm}$ with bandwidth of $1.0 \mathrm{~nm}$

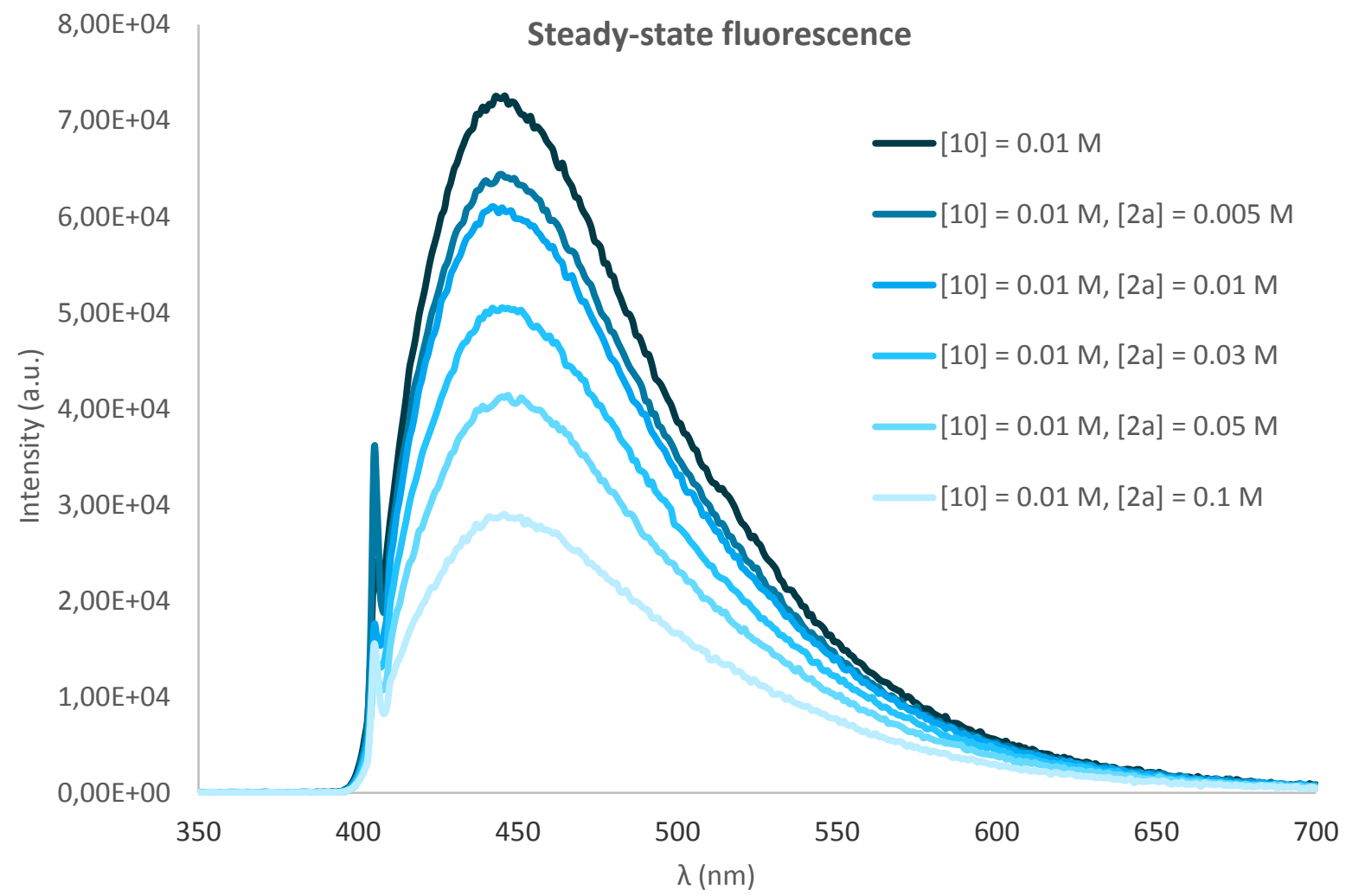

Figure S43: Luminescence of the BuNAH (10) in presence of different amount of redox-active ester $2 a$.

Table S15: Data of the Stern-Volmer quenching experiment of BuNAH (10) and redox-active ester $2 a$.

\begin{tabular}{|c|c|c|c|c|c|c|c|}
\hline & $\mathbf{2 a}(\mathrm{mmol})$ & $\mathbf{1 0}(\mathrm{mmol})$ & $\mathrm{V}(\mathrm{mL})$ & {$[\mathbf{2 a}]\left(\mathrm{Mx} \mathbf{1 0}^{-2}\right)$} & {$[\mathbf{1 0}](\mathrm{M})$} & $\mathrm{I}$ & $\mathrm{I} / \mathrm{I}$ \\
\hline 0 & 0.00 & 0.02 & 2.00 & 0 & 0.01 & 7.26 & 1.00 \\
\hline 1 & 0.01 & 0.02 & 2.00 & 0.50 & 0.01 & 6.41 & 1.13 \\
\hline 2 & 0.02 & 0.02 & 2.00 & 1.00 & 0.01 & 6.05 & 1.20 \\
\hline 3 & 0.06 & 0.02 & 2.00 & 3.00 & 0.01 & 5.04 & 1.44 \\
\hline 5 & 0.10 & 0.02 & 2.00 & 5.00 & 0.01 & 4.12 & 1.76 \\
\hline 5 & 0.20 & 0.02 & 2.00 & 10.00 & 0.01 & 2.90 & 2.50 \\
\hline
\end{tabular}




\section{Fluorescence lifetime}

\section{Stern-Volmer quenching experiment}

Inside the glove box, a dry cuvette was charged with freshly synthesized BuNAH (10; 0.02 $\mathrm{mmol}, 0.01 \mathrm{M}$ ) and DMSO (dry, degassed, spectroscopy grade) was added to adjust the total volume to $2 \mathrm{~mL}$. The cuvette was closed with a rubber septum. Every time the quenching of the BuNAH (10) by redox-active ester $2 \mathrm{a}$ was performed in a new cuvette. The redox-active ester $\mathbf{2 a}$ was added as indicated in table S3 and the excited state lifetime was measured by TCSPC using a $375 \mathrm{~nm}$ laser at $20 \mathrm{MHz}$ pulsing frequency with stop condition 10000 counts per channel (with emission at $\lambda=450 \mathrm{~nm}$ at a bandwidth of $5.0 \mathrm{~nm}$ ). The time domain decay was fitted using the exponential decay ( $\tau$, table S3). LUDOX ${ }^{\circledR}$ SM colloidal silica (30 wt. \% suspension in $\mathrm{H}_{2} \mathrm{O}$ ) was used to measure the instrument response function (IRF).

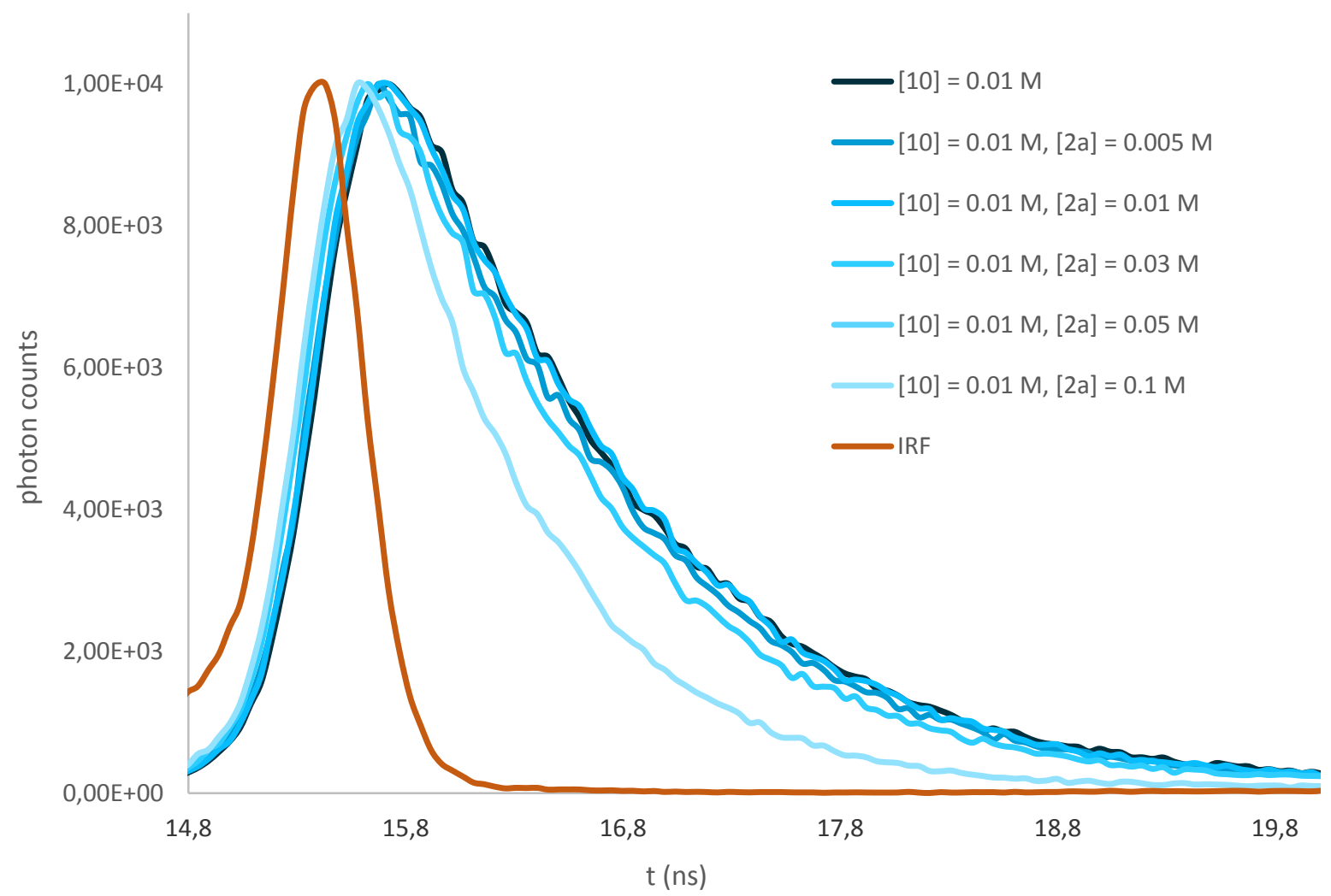

Figure S44: Lifetime measurement of BuNAH (10) in presence of different amount of the redox-active ester $\mathbf{2 a}$. 
Table S16: Data of the Stern-Volmer quenching experiment of BuNAH (10) and redox-active ester $\mathbf{2 a}$

\begin{tabular}{|c|c|c|c|c|c|c|c|c|}
\hline & $\mathbf{2 a}(\mathrm{mmol})$ & $\mathbf{1 0}(\mathrm{mmol})$ & $\mathrm{V}(\mathrm{mL})$ & {$[\mathbf{2 a}]\left(\mathrm{Mx} 10^{-2}\right)$} & {$[\mathbf{1 0}](\mathrm{M})$} & $\tau(\mathrm{ns})$ & $\chi^{2}$ & $\tau_{0} / \tau$ \\
\hline 0 & 0.00 & 0.02 & 2.00 & 0 & 0.01 & 1.08 & 1.28 & 1.00 \\
\hline 1 & 0.01 & 0.02 & 2.00 & 0.50 & 0.01 & 1.05 & 1.64 & 1.03 \\
\hline 2 & 0.02 & 0.02 & 2.00 & 1.00 & 0.01 & 1.06 & 1.80 & 1.02 \\
\hline 3 & 0.06 & 0.02 & 2.00 & 3.00 & 0.01 & 0.98 & 1.36 & 1.10 \\
\hline 4 & 0.10 & 0.02 & 2.00 & 5.00 & 0.01 & 0.86 & 1.49 & 1.26 \\
\hline 5 & 0.20 & 0.02 & 2.00 & 10.00 & 0.01 & 0.70 & 1.59 & 1.54 \\
\hline
\end{tabular}

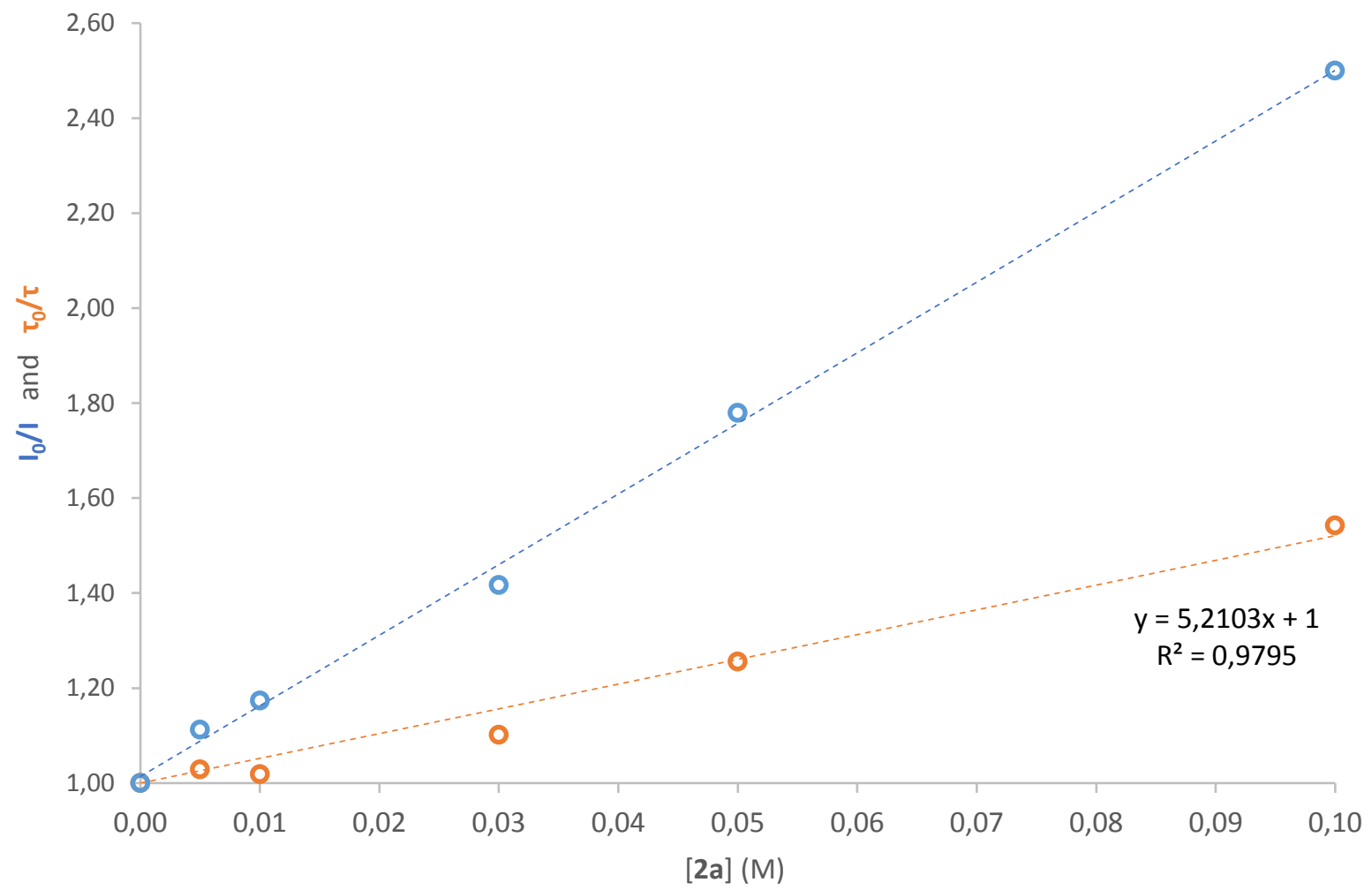

Figure S45: Stern-Volmer quenching of BuNAH (10) and redox-active ester 2a: steady-state data (blue; see Table S15) and time-resolved data (orange; see Table S16). The different slopes of steady-state and time-resolved data is clearly not consistent with either dynamic or static quenching mechanisms. 
Mixed static and dynamic quenching model

From the above figure, different slopes of lifetime measurement and steady state quenching experiment suggest the occurrence of both dynamic and static quenching with quenching constant $k_{q}$ and $K_{\text {eq }}$ respectively. The reason behind the static quenching can be attributed to the formation of a non-emissive EDA complex (see Figure S40 for UV-Vis enhanced absorption) between BuNAH (10) and redox-active ester $\mathbf{2 a}$.

The following figure can be considered to explain the dynamic and static quenching of BuNAH (10) by $2 a^{16}$

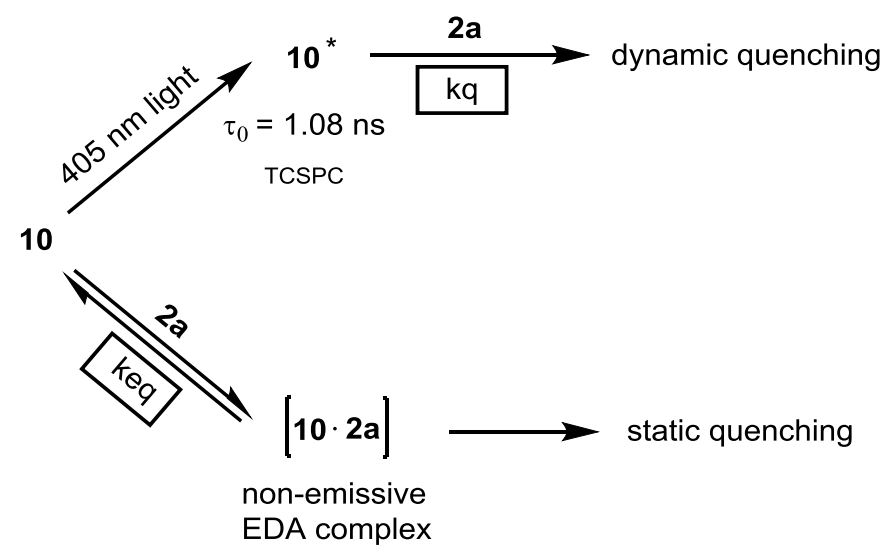

Figure S46: Model of dynamic and static quenching of BuNAH (10) in presence of redox-active ester $2 a$.

Considering this model, the steady state quenching intensities correspond to dynamic and static components, as follows:

$$
\frac{I_{0}}{I}=\left(1+k_{q} \tau_{0}[\mathbf{2} \mathbf{a}]\right) *\left(1+K_{e q}[\mathbf{2} \mathbf{a}]\right)
$$

\section{Rate of dynamic quenching}

Using the lifetime data (see Figure S22), we can determine the kinetics of the dynamic quenching component, using the Stern-Volmer expression:

$$
\frac{\tau_{0}}{\tau}=1+k_{q} \tau_{0}[\mathbf{2 a}]
$$

Where $\mathrm{k}_{\mathrm{q}}$ is the quenching constant and $\tau_{0}$ is the lifetime of BuNAH (10) without the quencher. The fluorescence lifetime of the pure BuNAH (10) was measured, $\tau_{0}=1.08$ ns (see Table S16, entry 0). From the linear regression of the time-resolved data (see Figure S45), we obtain 5.21 $=\mathrm{k}_{\mathrm{q}} \mathrm{T}_{0}$.

Thus, the quenching constant of the dynamic component is

$$
k_{q}=\frac{5.21}{\tau_{0}}=\frac{5.21}{1.08 * 10^{-9} S}=4.82 * 10^{9} M^{-1} s^{-1}
$$




\section{Fitting of the non-emissive EDA complex association constant:}

With this information in hand, the steady-state data was fitted using a mixed dynamic/static quenching model, where the equilibrium constant $\left(\mathrm{K}_{\text {eq }}\right)$ for the formation of the dark complex can be optimized to best fit the data from the equation below. This way, a value of $K_{\text {eq }}=6.75$ was estimated (see Figure S47). This value should only be considered as a rough estimation of the equilibrium constant.

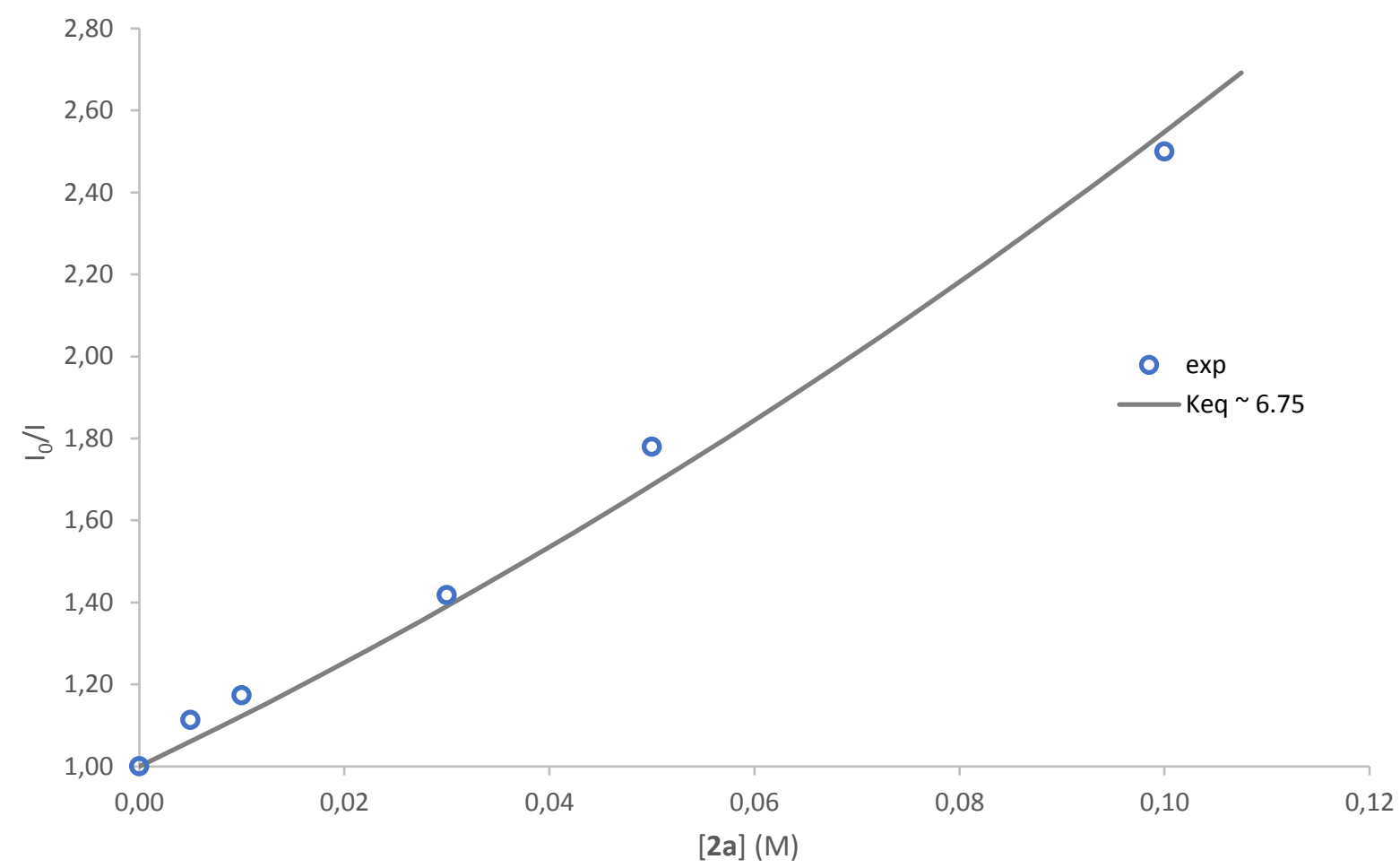

Figure S47: Fitting of the steady-state luminescence quenching data of BuNAH (10) with a model including static and dynamic quenching. 


\section{References}

1. Fawcett, A.; Pradeilles, J.; Wang, Y.; Mutsuga, T.; Myers, E. L.; Aggarwal, V. K. Science, 2017, $357,283-286$.

2. Pratsch, G.; Lackner, G. L.; Overman, L. E.; J. Org. Chem. 2015, 80, 6025-6036.

3. Lu, X.; Xiao, B.; Liu, L.; Fu, Y. Chem. Eur. J. 2016, 22, 11161-11164.

4. Shen, M, L.; Shen, Y.; Wang, P. S. Org. Lett. 2019, 21, 2993-2997.

5. Qin, T.; Malins, L. R.; Edwards, J. T.; Merchant, R. R.; Novak, A. J. E.; Zhong, J. Z.; Mills, R. B.; Yan, M.; Yuan, C.; Eastgate, M. D.; Baran, P. S. Angew. Chem. Int. Ed. 2017, 56, $260-265$.

6. Xue, W.; Oestreich, M. Angew. Chem. Int. Ed. 2017, 56, 11649-11652.

7. Gao, L.; Wang, G.; Cao, J.; Yuan, D.; Cheng, X.; Guo, X.; Lia, S. Chem. Commun. 2018, 54, 11534-11537.

8. Nawrat, C. C.; Jamison, C. R.; Slutskyy, Y.; MacMillan, D. W. C.; Overman, L. E. J. Am. Chem. Soc. 2015, 137, 11270-11273.

9. Lackner, G. L.; Quasdorf, K. W.; Overman, L. E. J. Am. Chem. Soc. 2013, 135, 15342-15345.

10. Zheng, C.; Wang, G. Z.; Shang, R. Adv. Synth. Catal. 2019, 361, $4500-4505$.

11. ElMarrouni, A.; Ritts. C. B.; Balsells. J. Chem. Sci., 2018, 9, 6639-6646.

12. Paul, C. E.; Gargiulo, S.; Opperman, D. J.; Lavandera, I.; Gotor-Fernandez, V.; Gotor, V.; Taglieber, A.; Arends, I. W. C. E.; Hollmann, F. Org. Lett. 2013, 15, 180-183.

13. Lackner, G. L.; Quasdorf, K. W.; Pratsch, G.; Overman, L. E. J. Org. Chem. 2015, 80, 6012-6024.

14. Geddes, A.; Paul, C. E.; Hay, S.; Hollmann, F.; Scrutton, N. S. J. Am. Chem. Soc. 2016, 138, 11089-11092.

15. Cismesiaa, M. A.; Yoon, T. P. Chem. Sci., 2015, 6, 5426-5434.

16. Fraiji, L. K.; Hayes, D. M.;1 Werner, T. C.; J. Chem. Educ. 1992, 69, 424-428.

17. Wang, J.; Lundberg, H.; Asai, S.; Martín-Acosta, P.; Chen, J. S.; Brown, S.; Farrell, W.; Dushin, R. G.; O’Donnell, C. J.; Ratnayake, A. S.; Richardson, P.; Liu, Z.; Qin, T.; Blackmond, D. G.; Baran, P. S., Proc. Natl. Acad. Sci. 2018, 115(28), E6404-E6410.

18 Kölmel, D. K.; Loach, R. P.; Knauber, T.; Flanagan, M. E., ChemMedChem 2018, 13, 2159-2165.

19 Ding, Y.; Franklin, G. J.; DeLorey, J. L.; Centrella, P. A.; Mataruse, S.; Clark, M. A.; Skinner, S. R.; Belyanskaya, S., ACS Comb. Sci. 2016, 18, 625-629.

20 Satz, A. L.; Cai, J.; Chen, Y.; Goodnow, R.; Gruber, F.; Kowalczyk, A.; Petersen, A.; NaderiOboodi, G.; Orzechowski, L.; Strebel, Q., Bioconjugate Chem. 2015, 26, 1623-1632. 


\section{NMR spectra of synthesised compounds}

${ }^{1} \mathrm{H}-\mathrm{NMR}\left(400 \mathrm{MHz}, \mathrm{CDCl}_{3}\right)$ for compound $2 q$

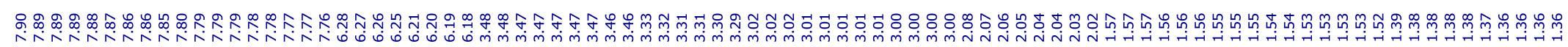

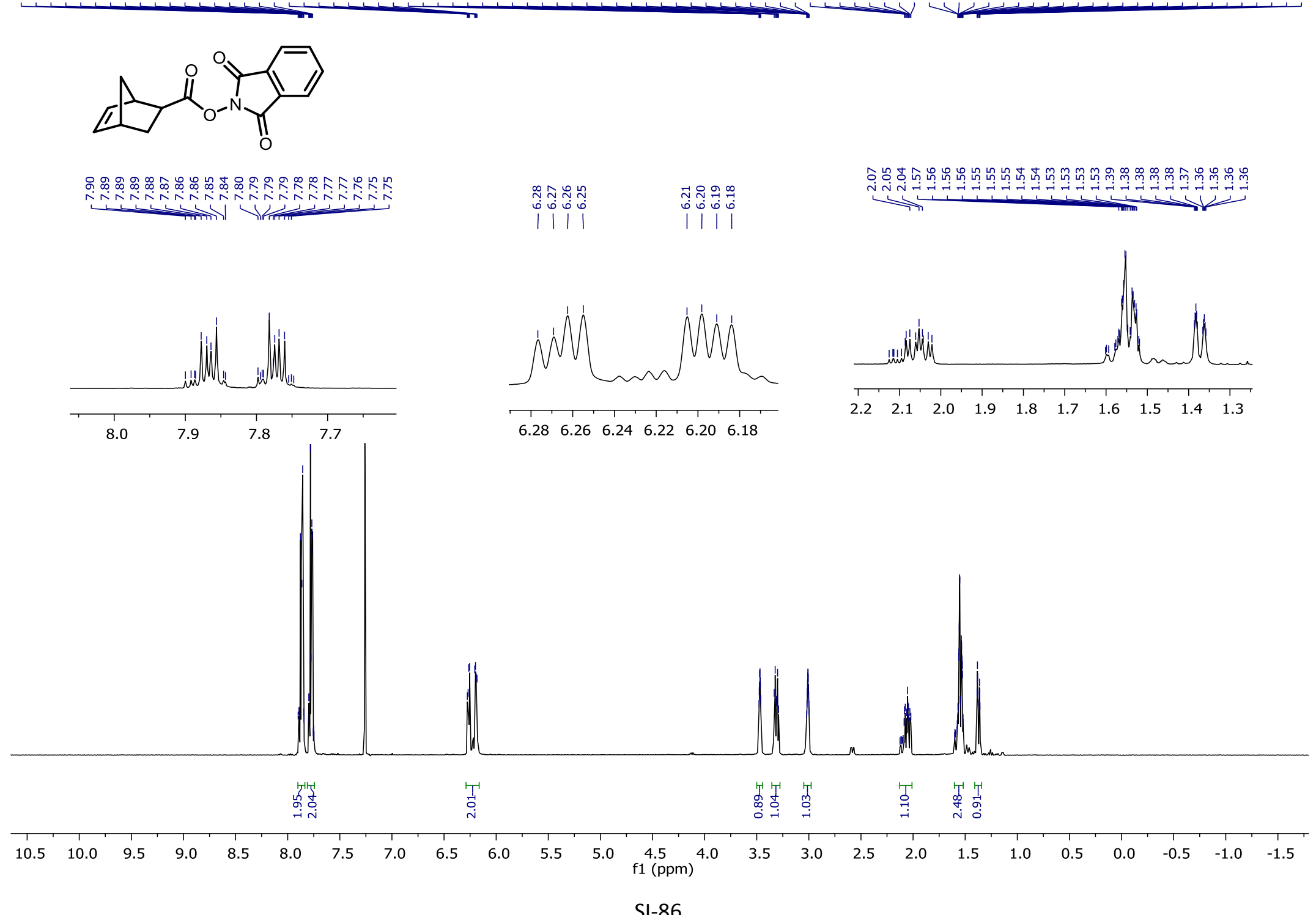


${ }^{13} \mathrm{C}-\mathrm{NMR}(101 \mathrm{MHz}, \mathrm{CDCl}$ ) for compound $2 q$

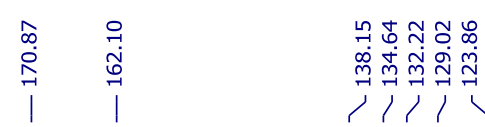

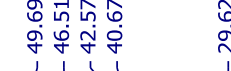

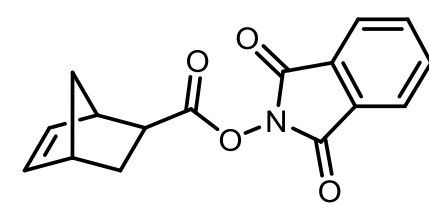

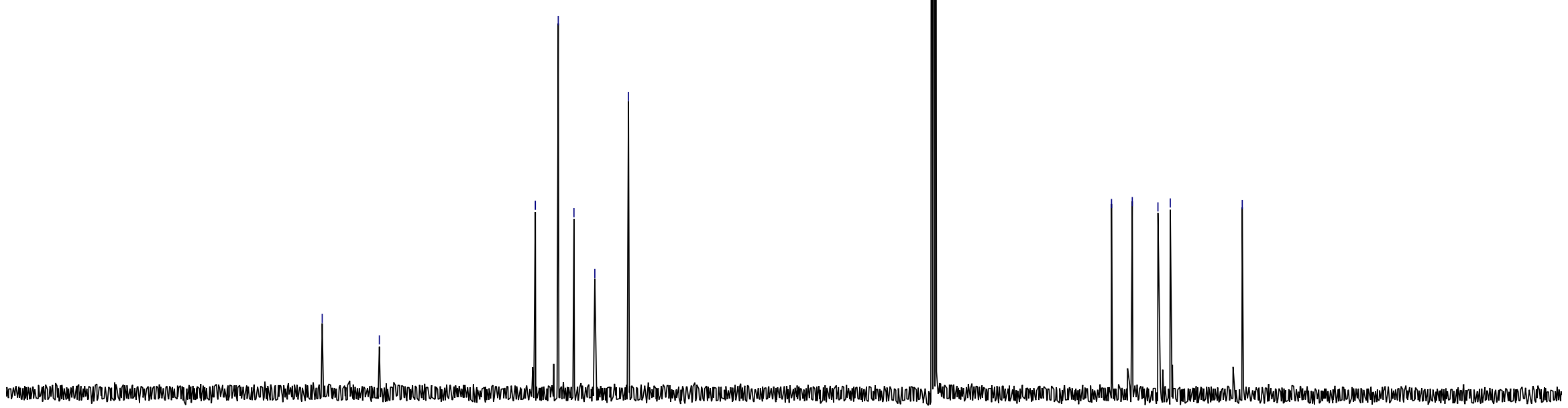




\section{${ }^{1} \mathrm{H}-\mathrm{NMR}\left(400 \mathrm{MHz}, \mathrm{CDCl}_{3}\right)$ for compound $2 r$}

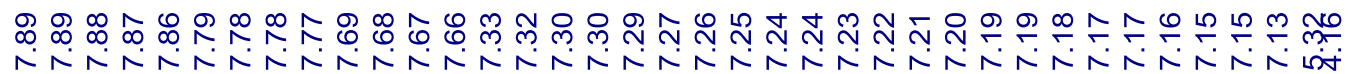

இ வ

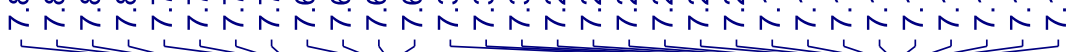
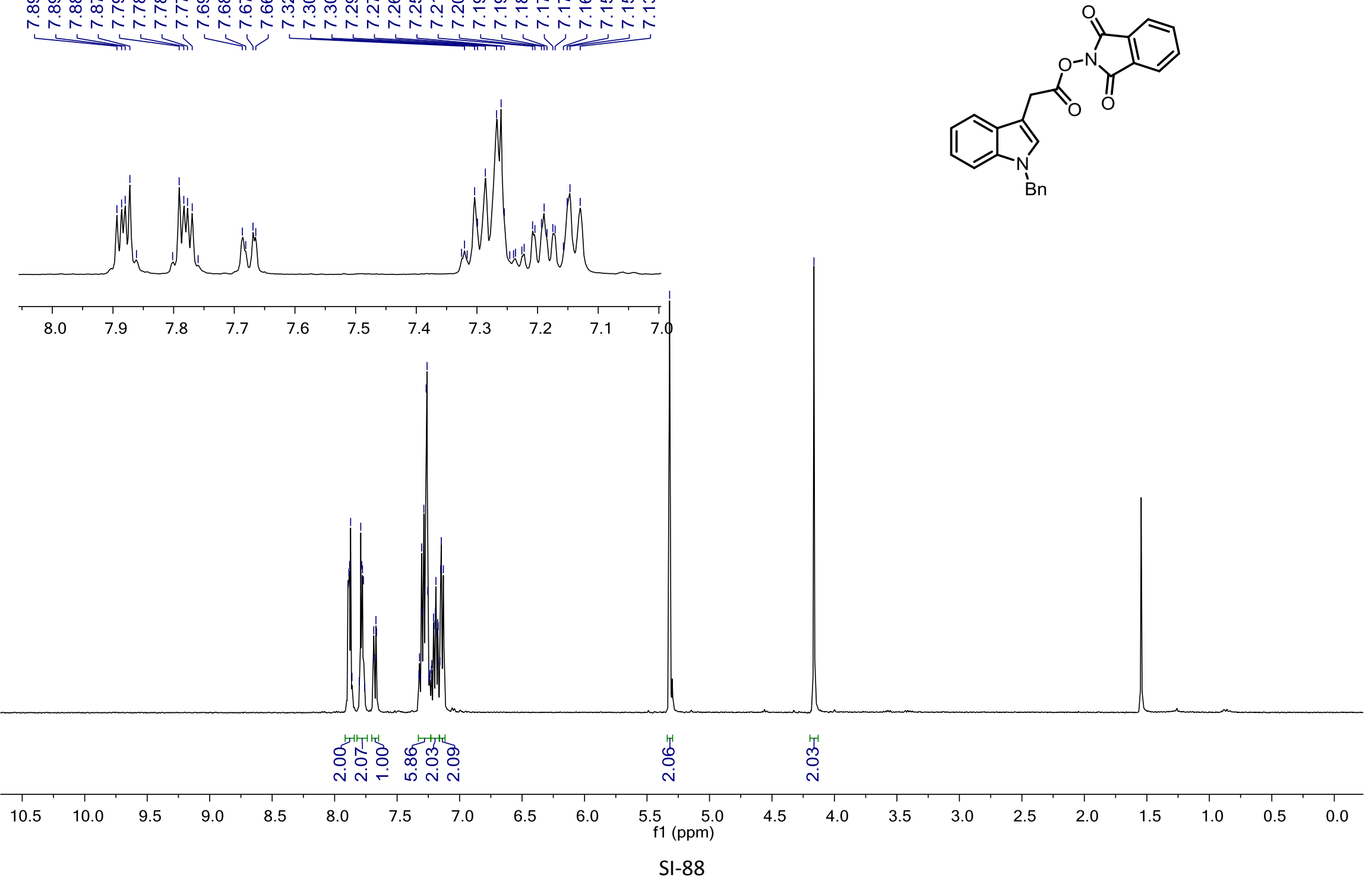
${ }^{13} \mathrm{C}-\mathrm{NMR}\left(101 \mathrm{MHz}, \mathrm{CDCl}_{3}\right)$ for compound $\mathbf{2 r}$

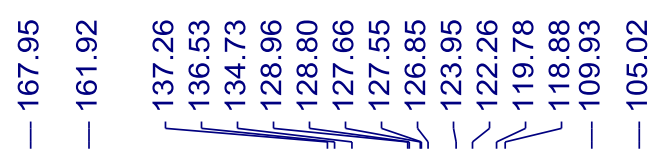

ㅇํㅇ

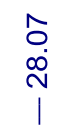

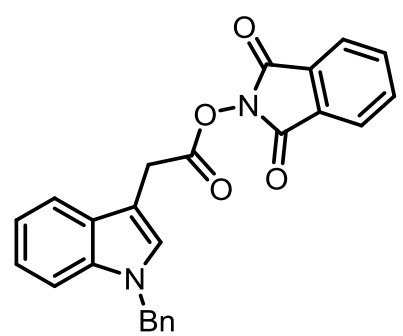

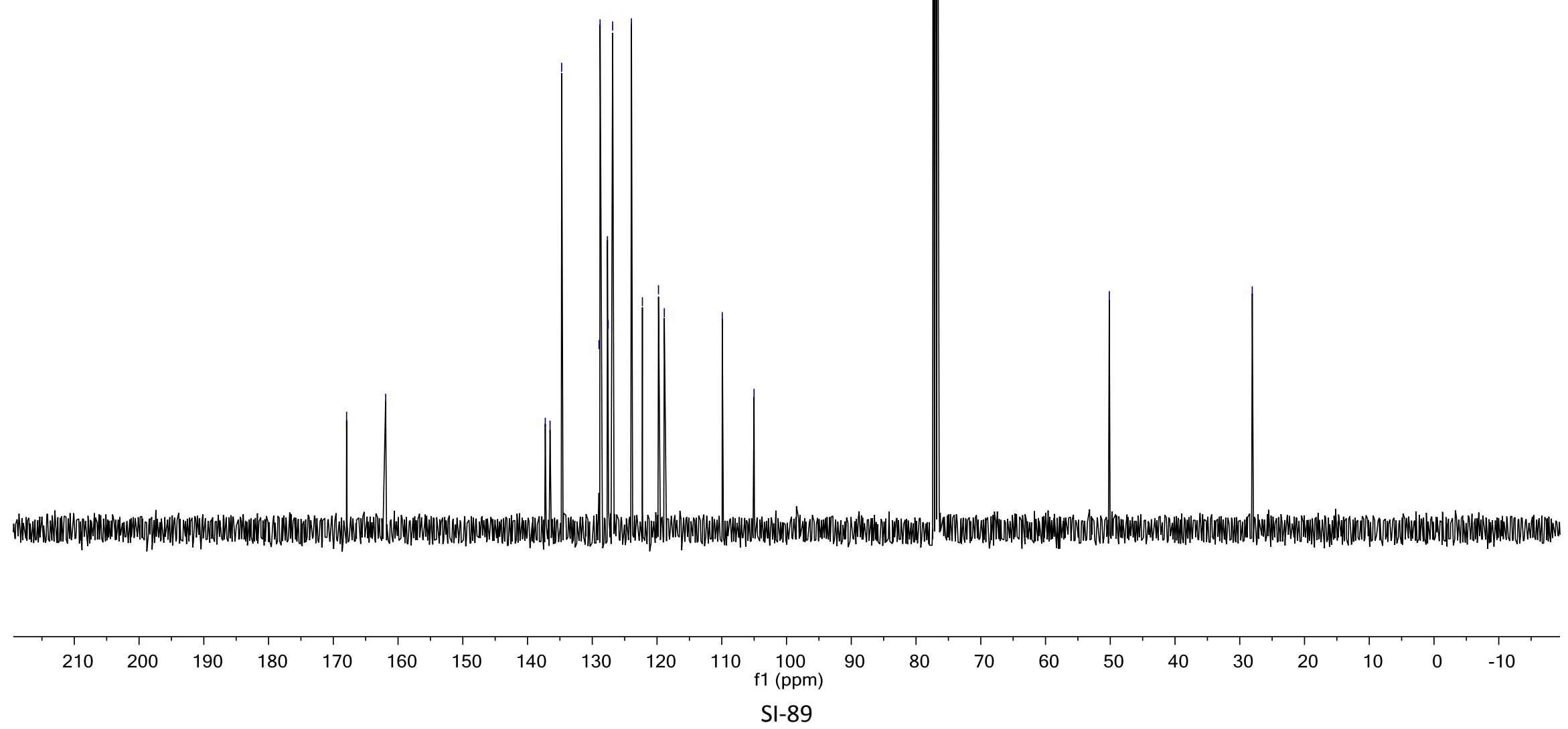




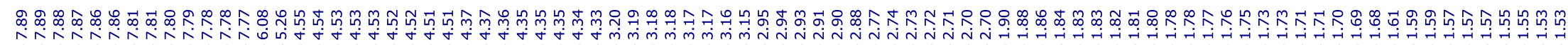

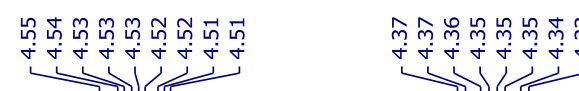

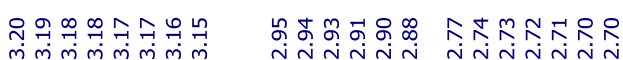

itits

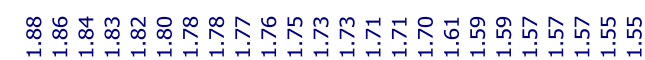

पाl?

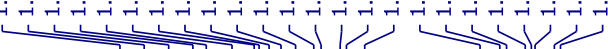
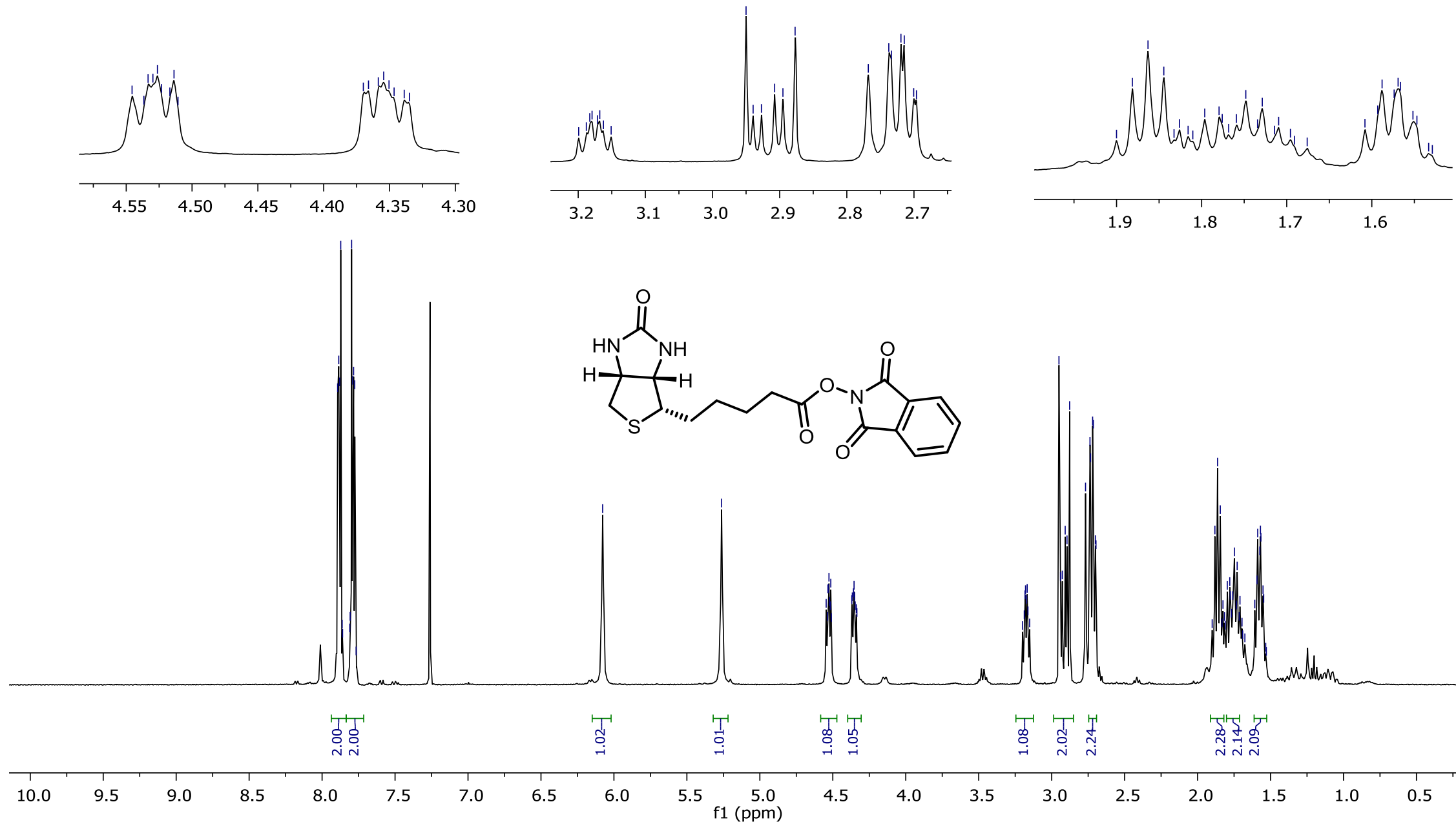
${ }^{13} \mathrm{C}-\mathrm{NMR}(101 \mathrm{MHz}, \mathrm{CDCl} / 3)$ for compound $2 \mathrm{~s}$

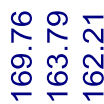
\&ธก

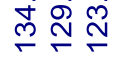
ํํำ
ㄴ.
1 >1
$>11$
ن<smiles>O=C1NC(=O)NC2C(CS[C@@H]2CCCCC(=O)ON2C(=O)c3ccccc3C2=O)N1</smiles>

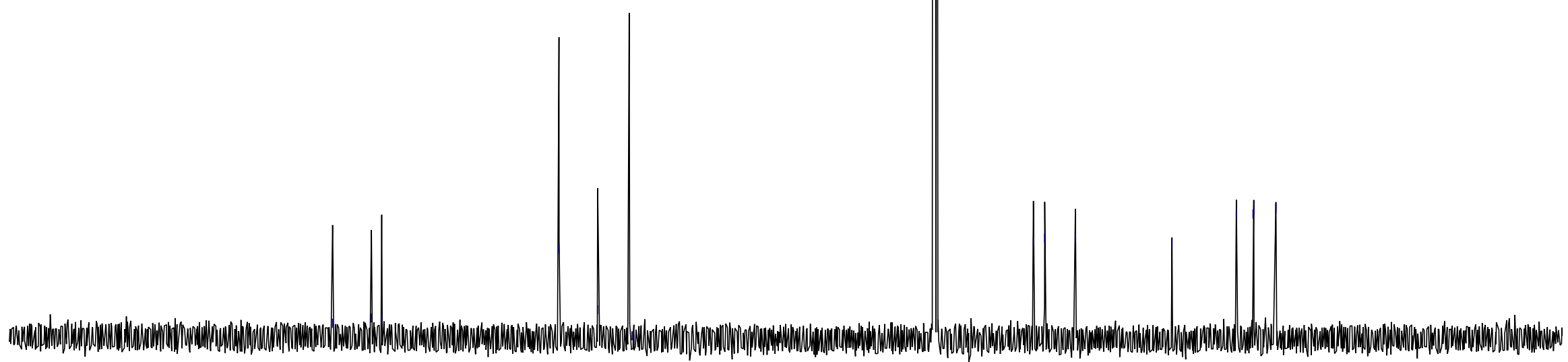
210
200
190
80
160
$150 \quad 140$
$130 \quad 120$
$110 \quad 100$

$90 \quad 80$

70

$60 \quad 50$

40

30

20 


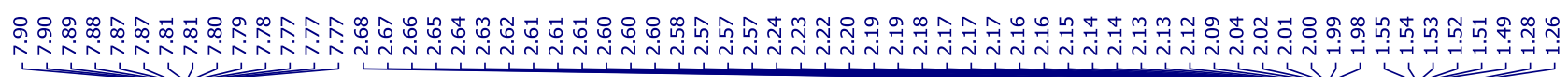

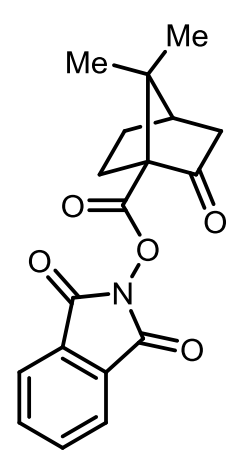

䎹

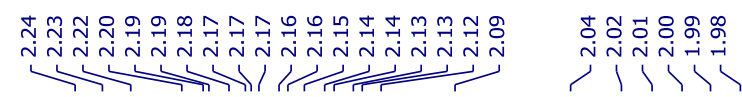
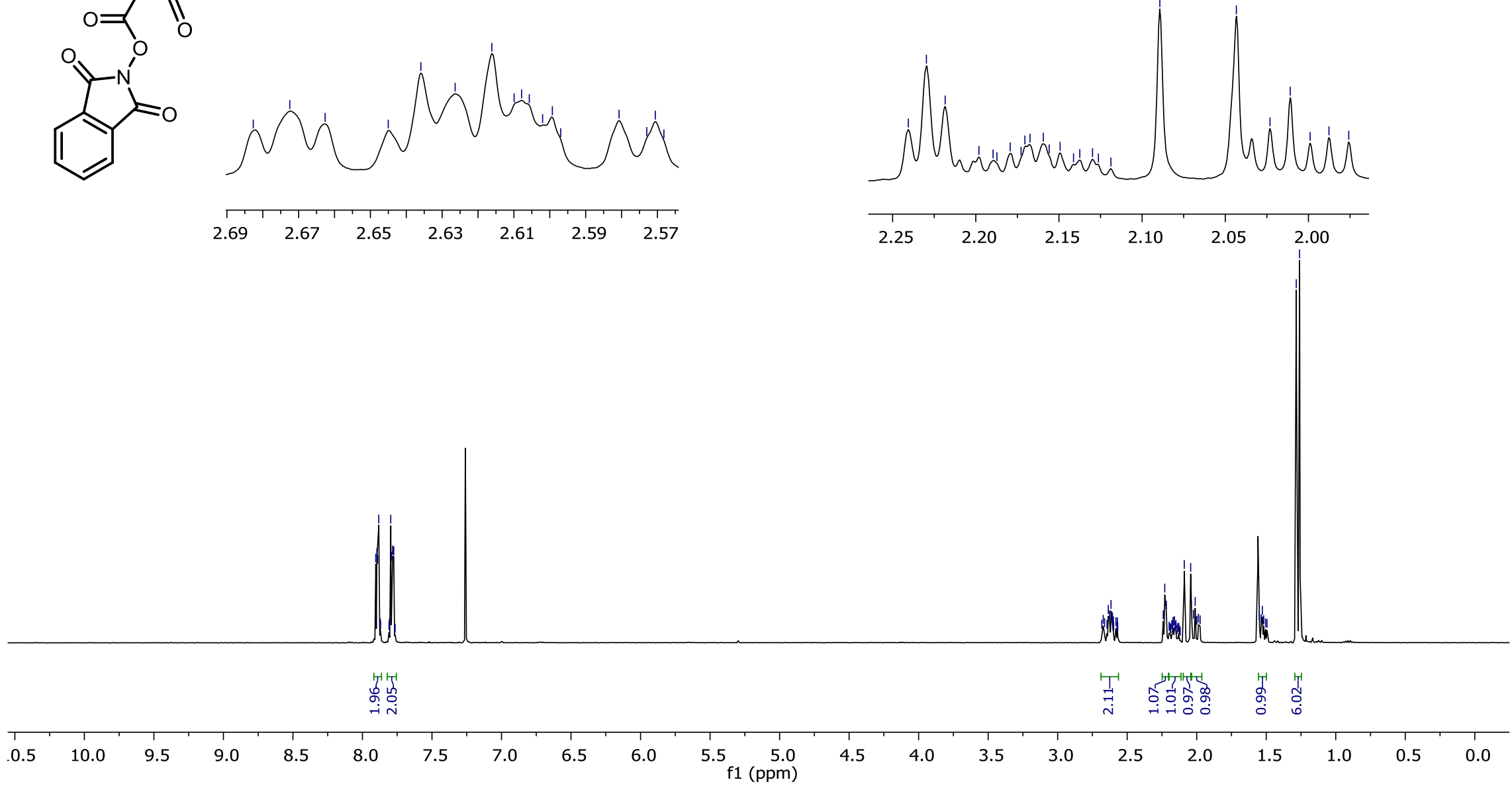
${ }^{13} \mathrm{C}-\mathrm{NMR}(101 \mathrm{MHz}, \mathrm{CDCl} / 3)$ for compound $\mathbf{2 x}$

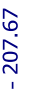

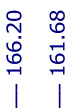

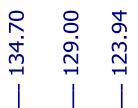
$\stackrel{\infty}{\infty}$

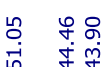
ํํㅇำำ
ㄴํㄴ은
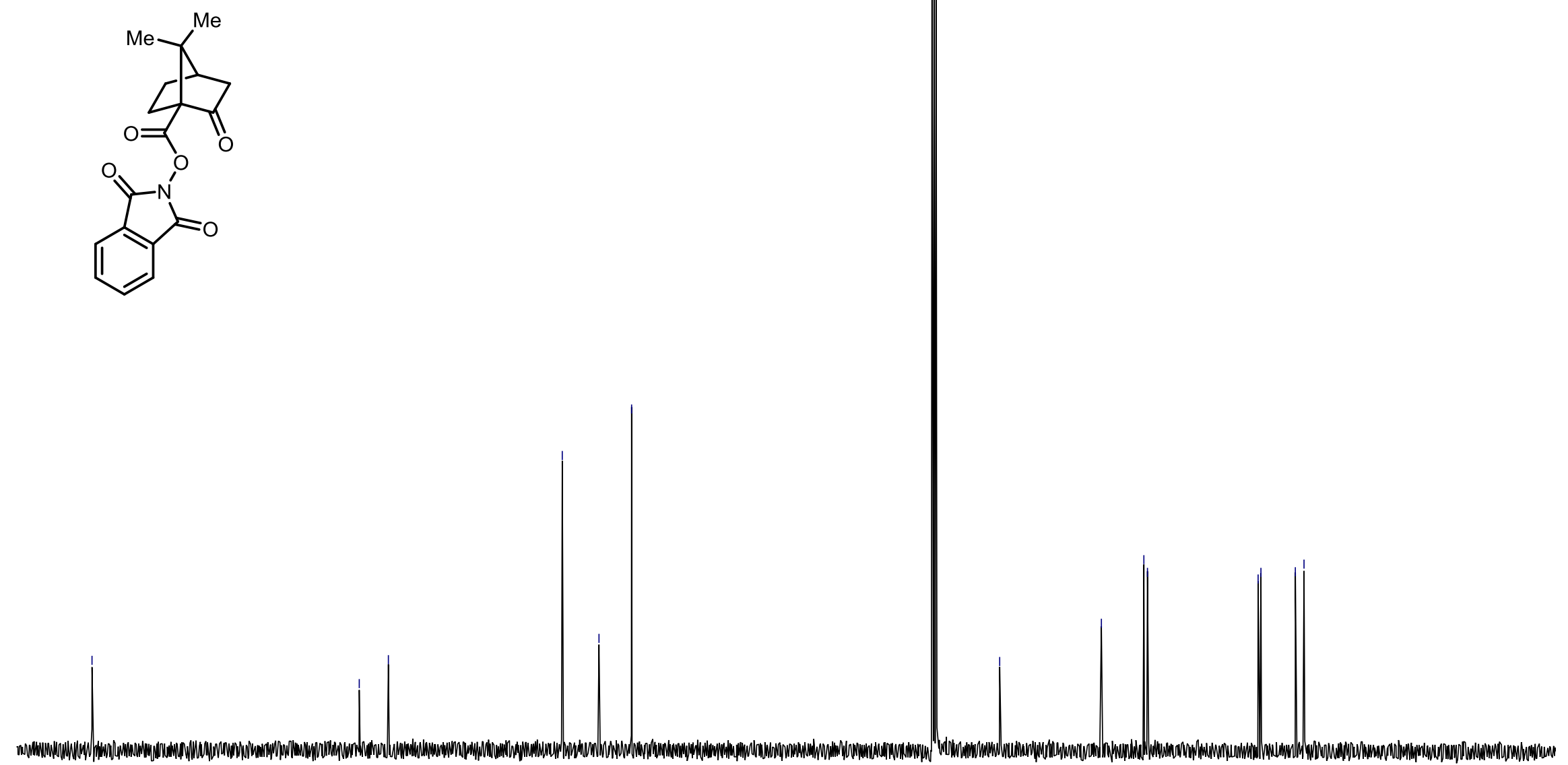

$210 \quad 200$ $190 \quad 180$ 160 $150 \quad 140$ $130 \quad 120$ $110 \quad 100$ 


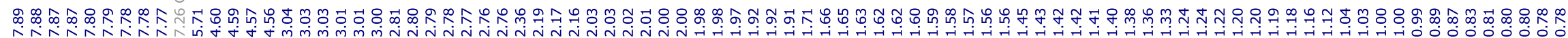
(1)

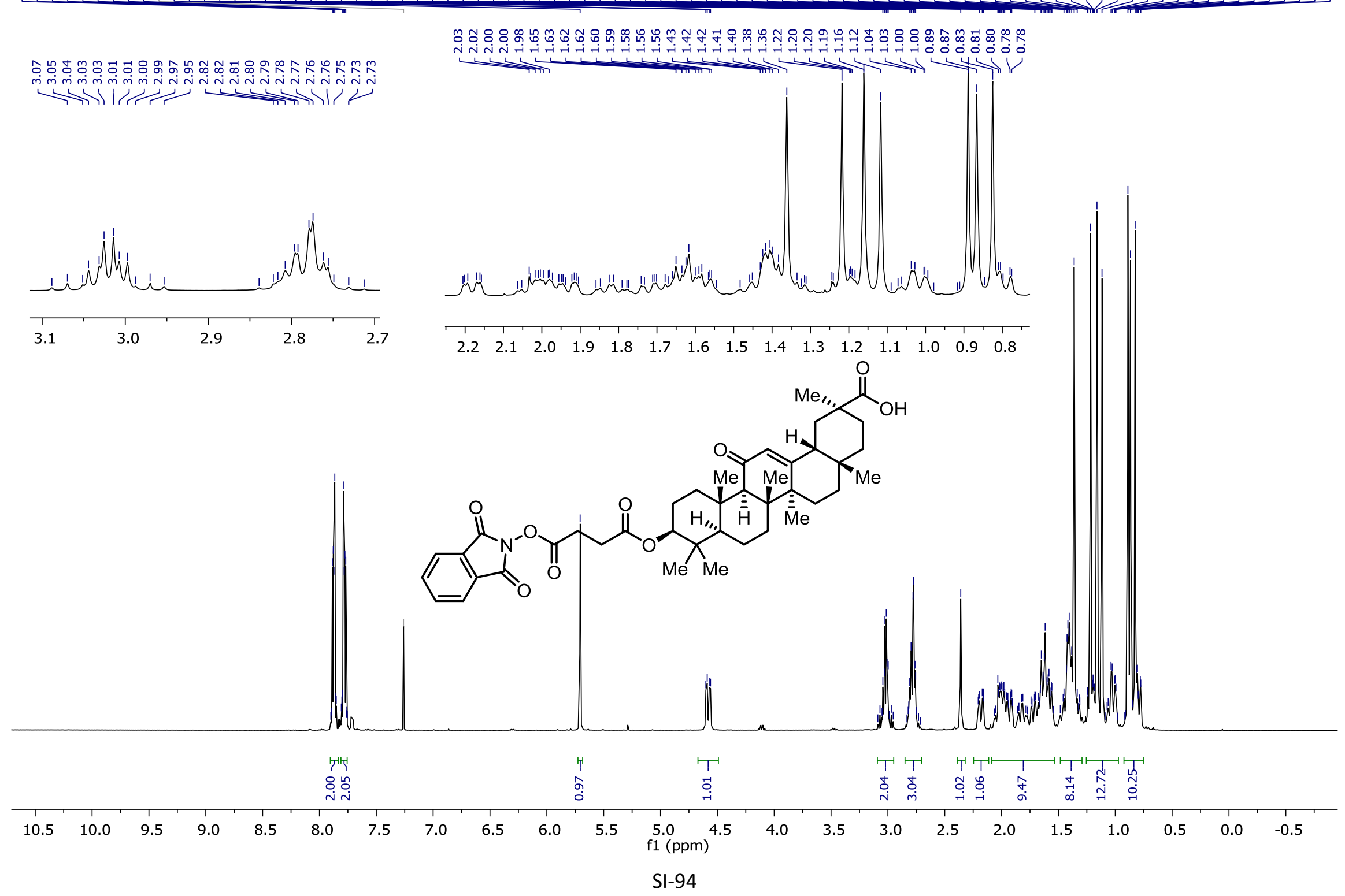


${ }^{13} \mathrm{C}-\mathrm{NMR}\left(101 \mathrm{MHz}, \mathrm{CDCl}_{3}\right)$ for compound $2 a a$

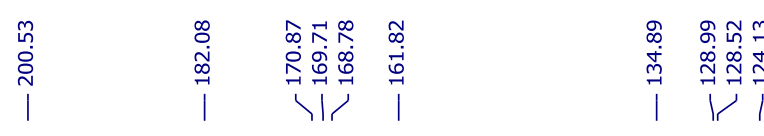

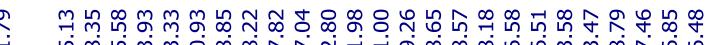

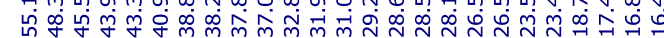

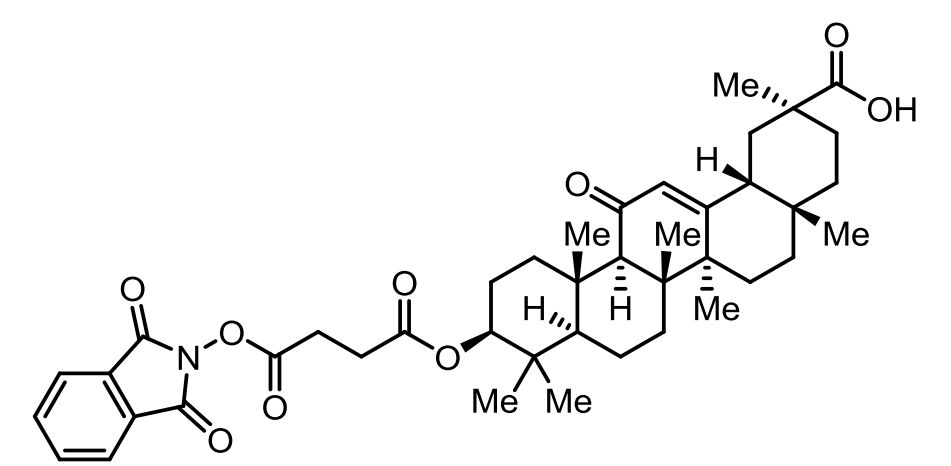

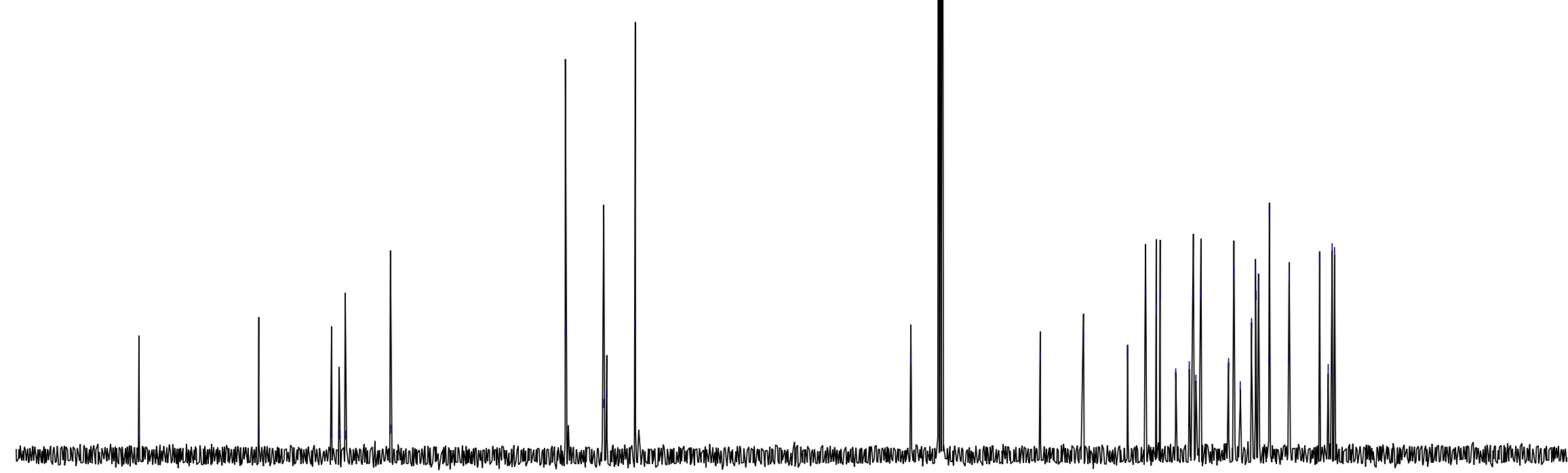
$180 \quad 170$ 
DEPT 135 (101 MHz, CDCl $)$ for compound 2aa

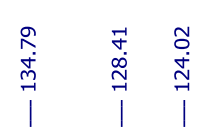
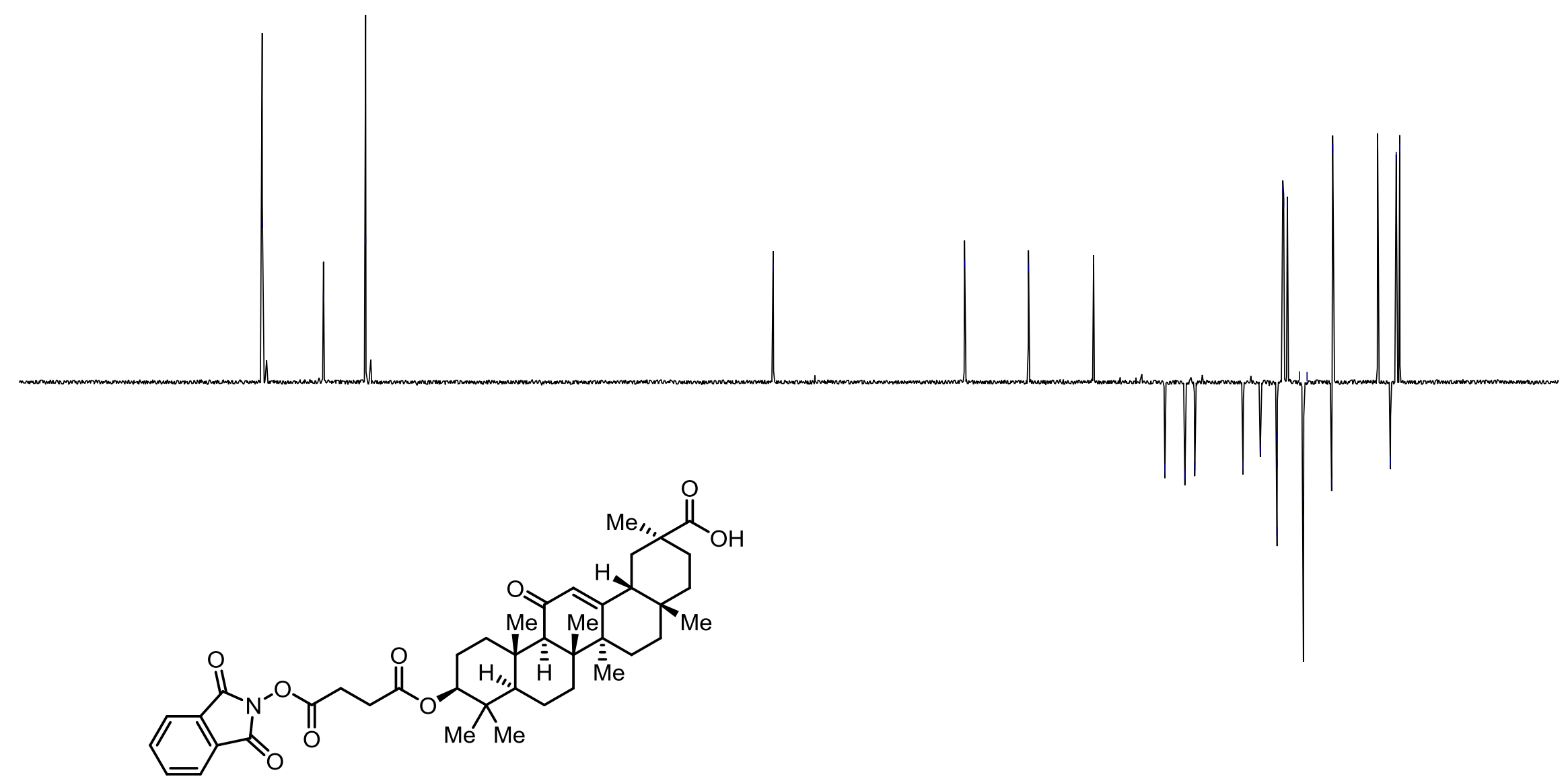


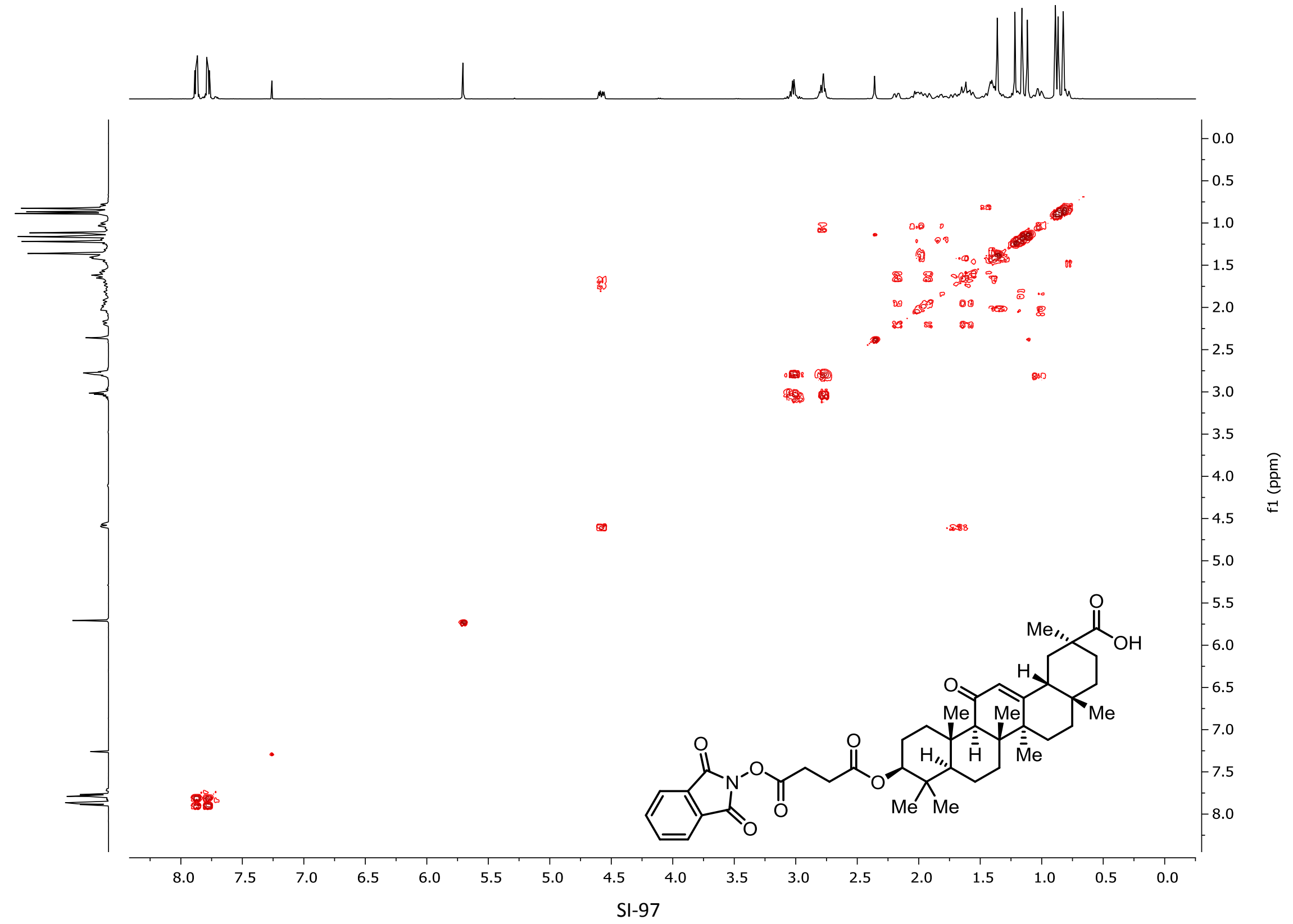




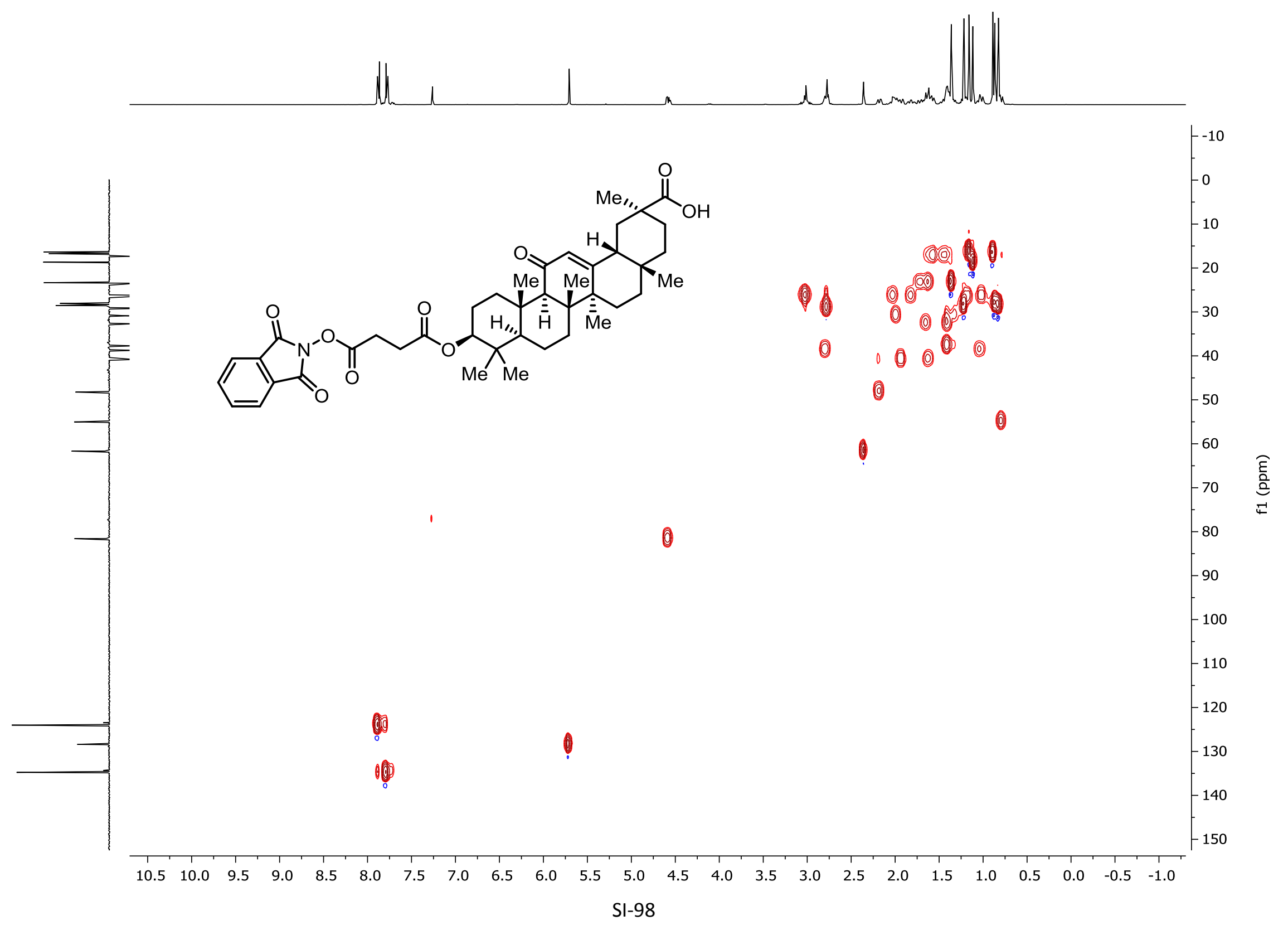




\section{${ }^{1} \mathrm{H}-\mathrm{NMR}\left(400 \mathrm{MHz}, \mathrm{CDCl}_{3}\right)$ for compound $4 \mathrm{a}$}

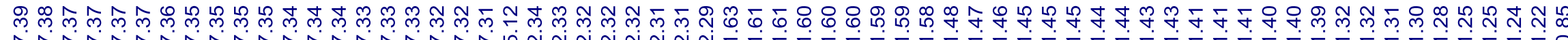

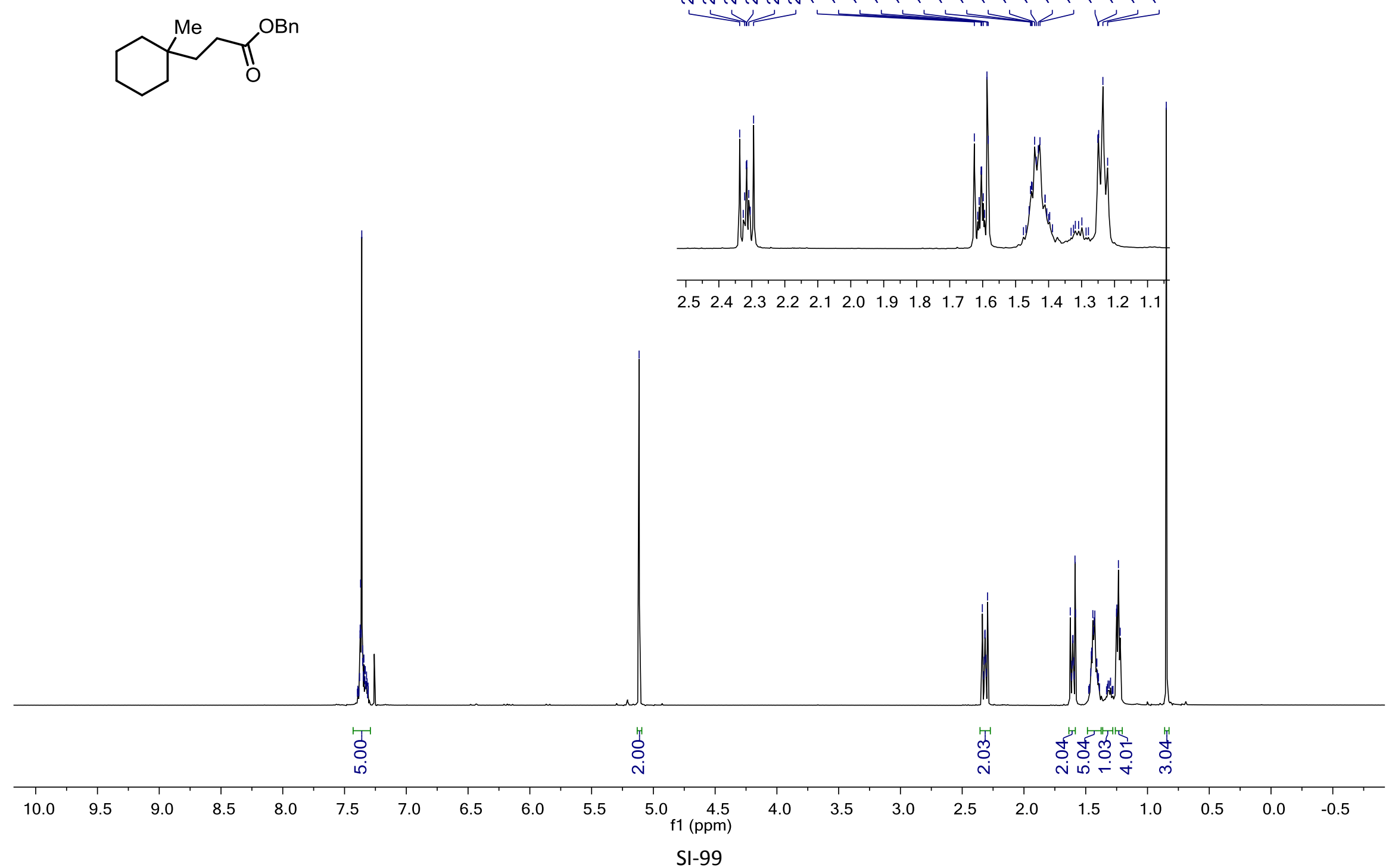

商 
${ }^{13} \mathrm{C}-\mathrm{NMR}\left(101 \mathrm{MHz}, \mathrm{CDCl}_{3}\right)$ for compound $\mathbf{4 a}$

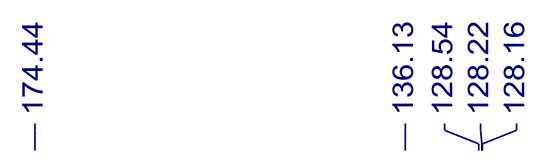

$\stackrel{0}{\dot{0}}$

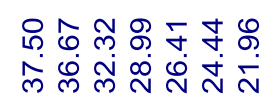

\& $\quad \sqrt{1} 1111$
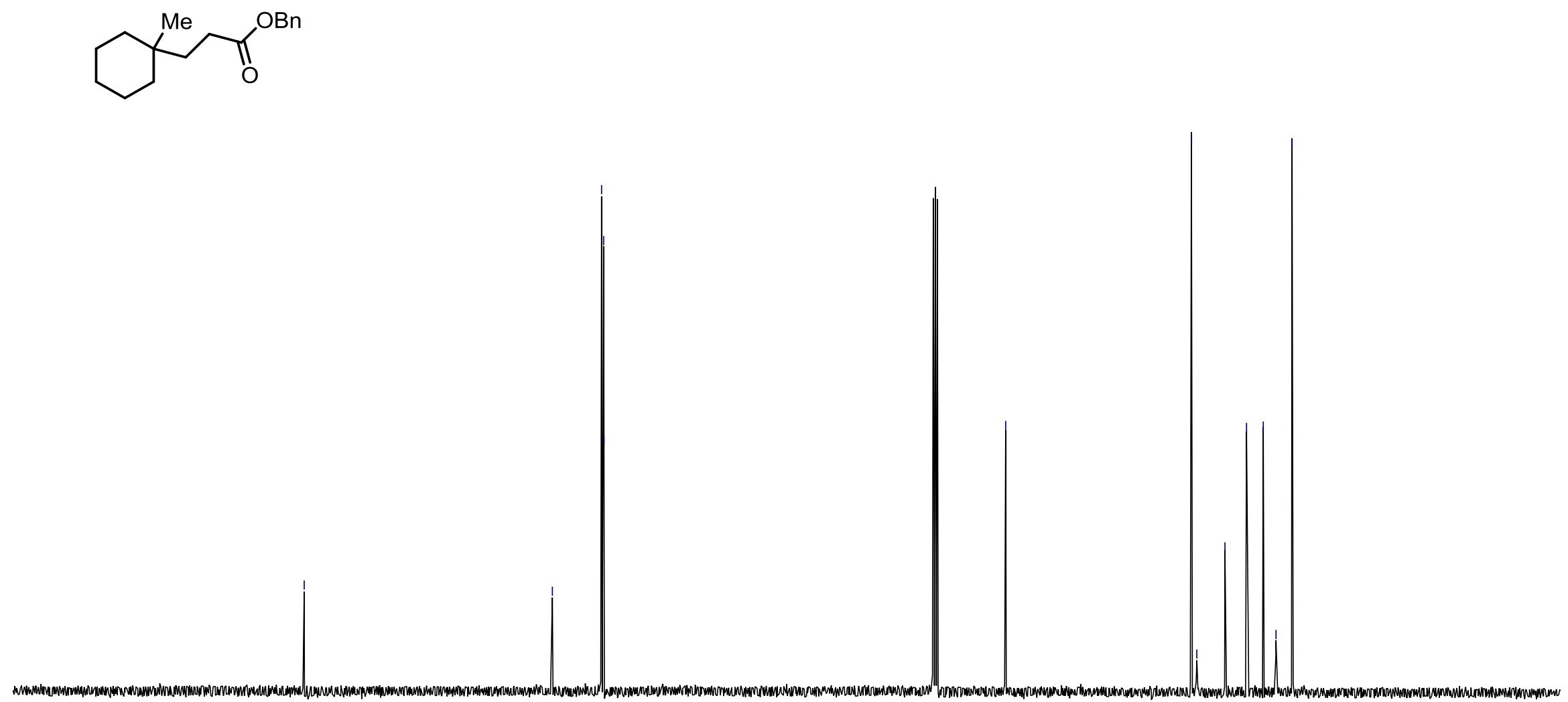

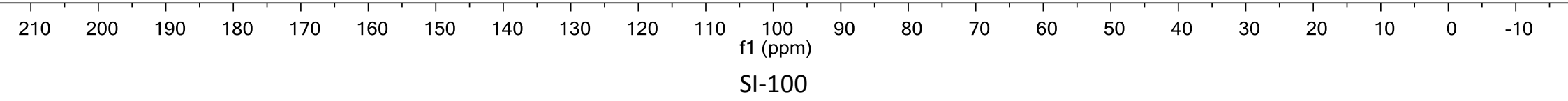




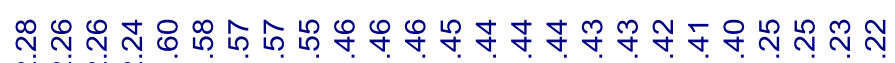
$\underbrace{\mathrm{n} u \mathrm{n}} \mathrm{i}$

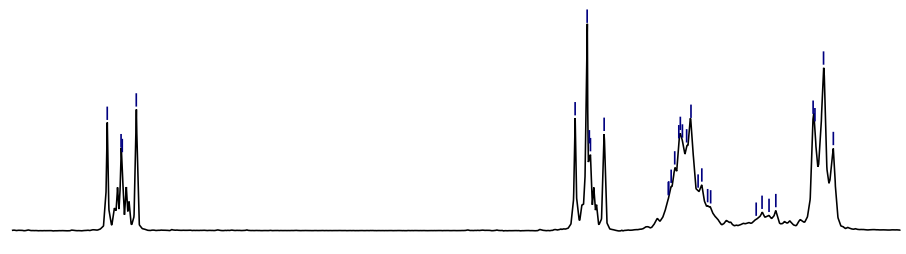

\begin{tabular}{llllllllllllllllll}
\hline 2.4 & 2.3 & 2.2 & 2.1 & 2.0 & 1.9 & 1.8 & 1.7 & 1.6 & 1.5 & 1.4 & 1.3 & 1.2
\end{tabular}

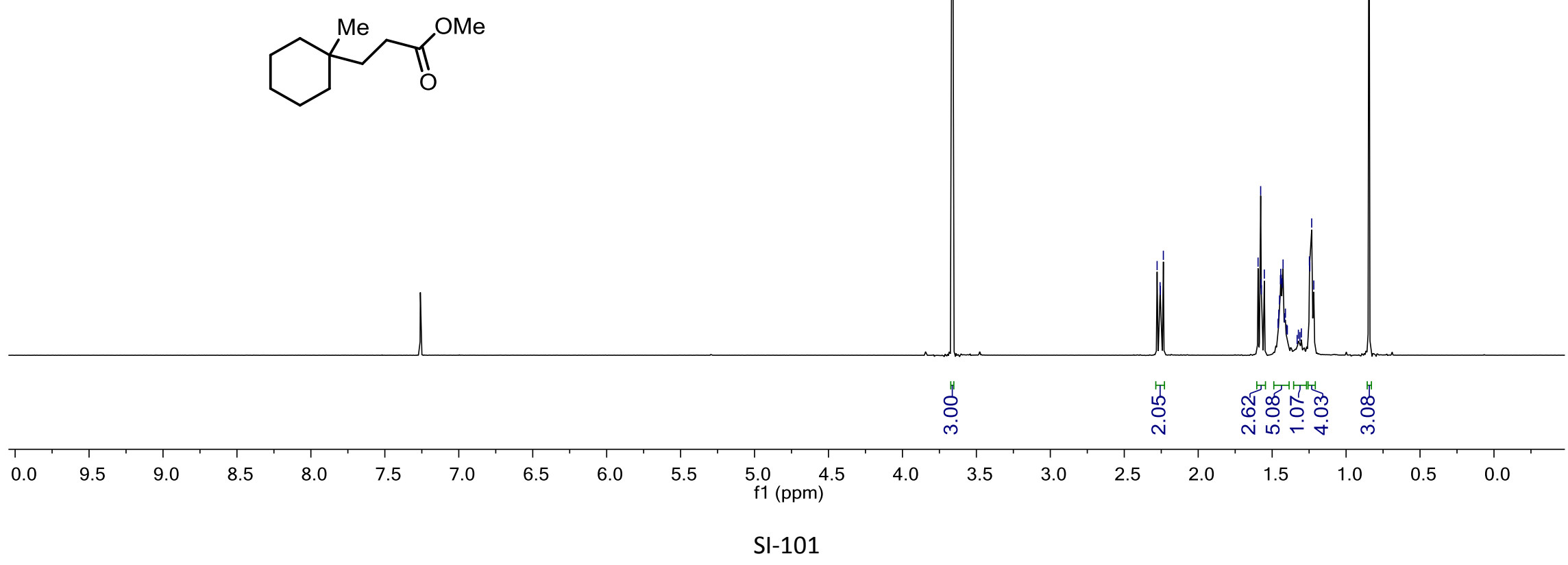

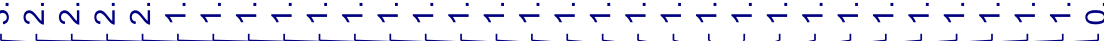


${ }^{13} \mathrm{C}-\mathrm{NMR}(101 \mathrm{MHz}, \mathrm{CDCl} / 3)$ for compound $\mathbf{4 b}$

$\frac{8}{\circ}$

in $\quad$ in

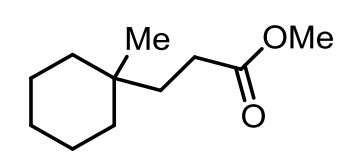

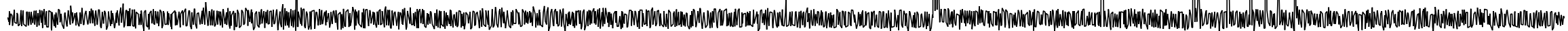

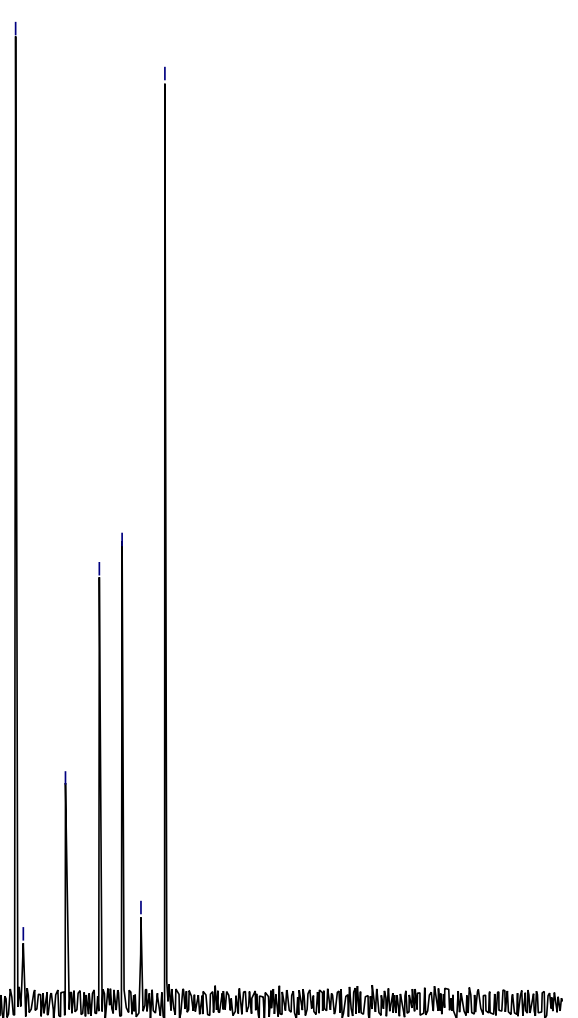

$\begin{array}{llllllllllll}210 & 200 & 190 & 180 & 170 & 160 & 150 & 140 & 130 & 120 & 110 & 100 \\ & & & & & & & \end{array}$ 


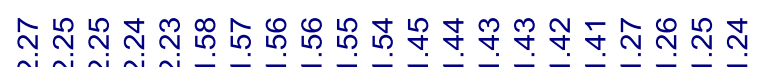
inn
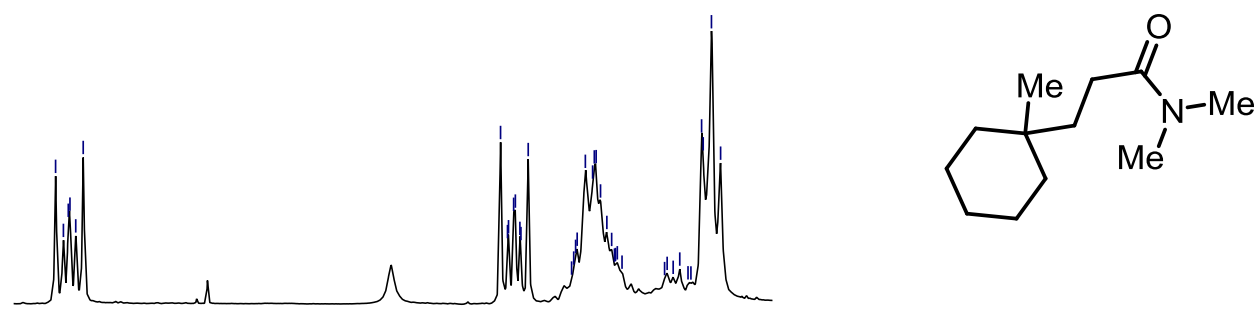

$\begin{array}{llllllllllllll}2.3 & 22 & 21 & 2.0 & 1.9 & 1.8 & 1.7 & 1.6 & 1.5 & 1.4 & 1.3 & 1.2\end{array}$

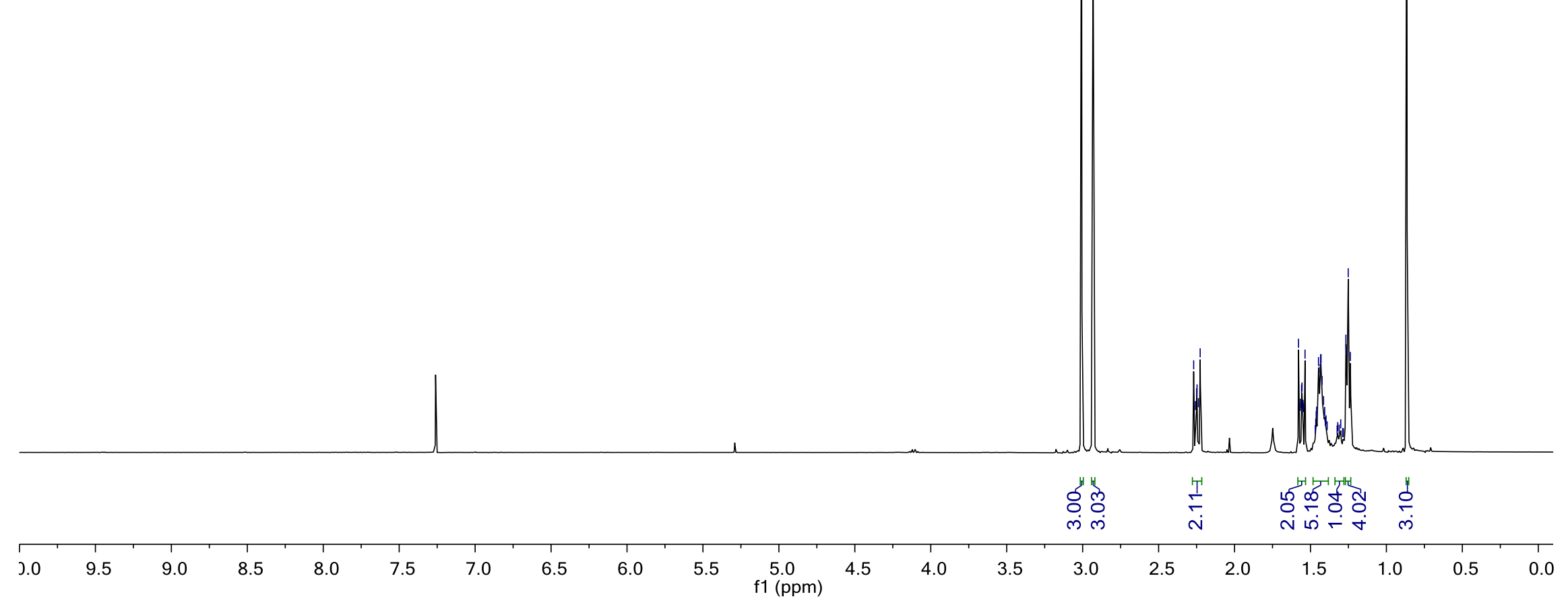

SI-103 
${ }^{13} \mathrm{C}-\mathrm{NMR}\left(101 \mathrm{MHz}, \mathrm{CDCl}_{3}\right)$ for compound $4 \mathrm{C}$

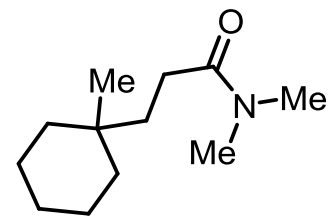

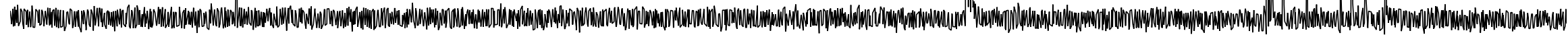

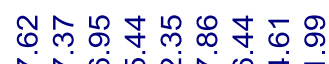

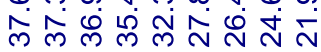
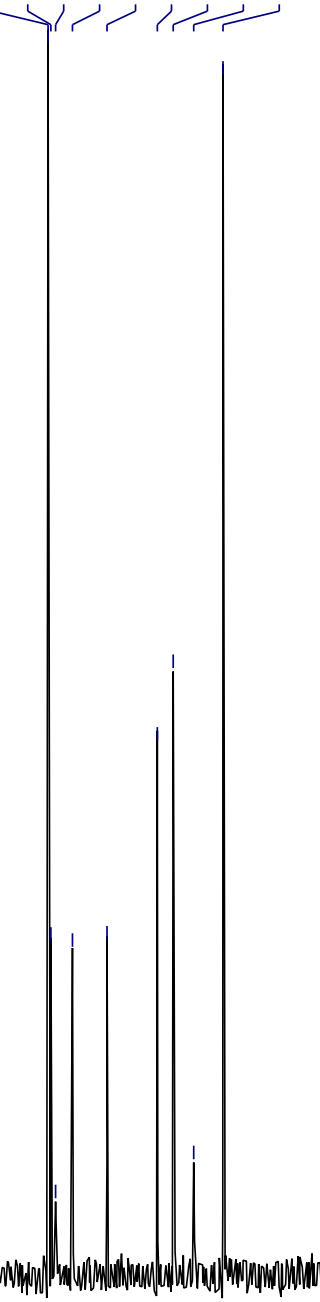

$$
200
$$

190

180

170

160

150

140

130

120

110

100
$\mathrm{f} 1(\mathrm{ppm})$

90

80

70

60

50

40

30

20 


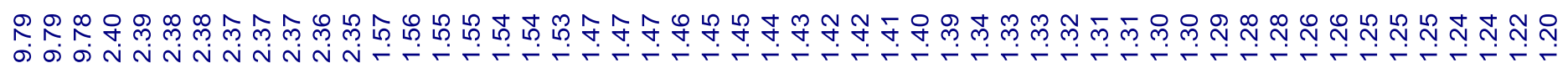
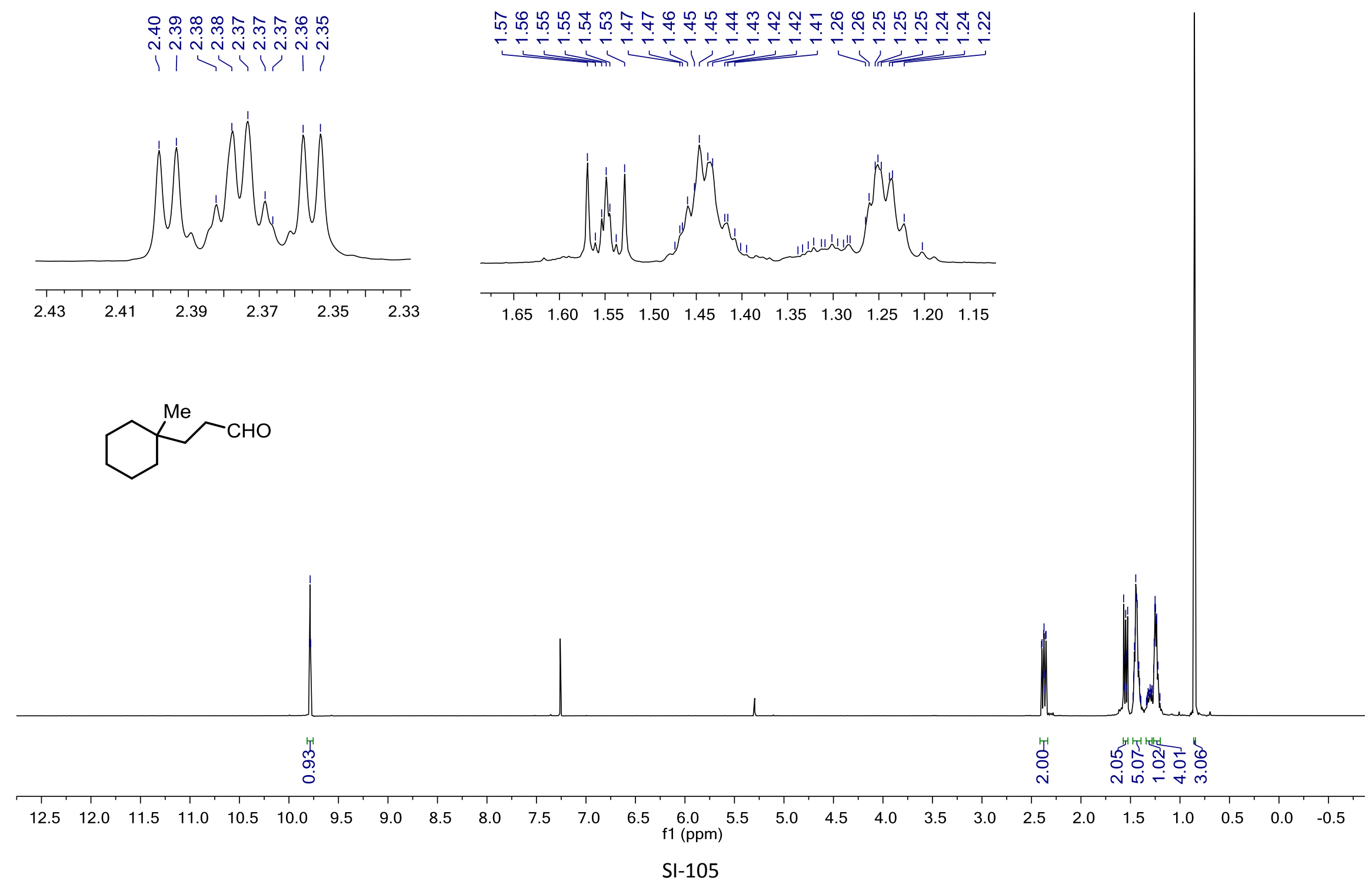
${ }^{13} \mathrm{C}-\mathrm{NMR}(101 \mathrm{MHz}, \mathrm{CDCl}$ ) for compound 4d

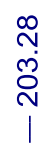

กำ

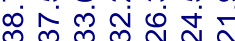

$11<1<1$

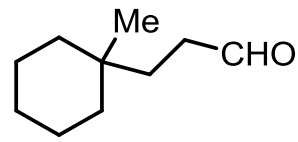

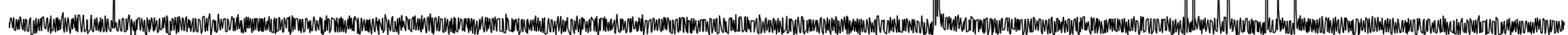

\begin{tabular}{|c|c|c|c|c|c|c|c|c|c|c|c|c|c|c|c|c|c|c|c|c|c|c|}
\hline 210 & 200 & 190 & 180 & 170 & 160 & 150 & 140 & 130 & 120 & 110 & $\begin{array}{c}100 \\
\mathrm{f} 1(\mathrm{ppm})\end{array}$ & 90 & 80 & 70 & 60 & 50 & 40 & 30 & 20 & 10 & 0 & -10 \\
\hline & & & & & & & & & & & SI-106 & & & & & & & & & & & \\
\hline
\end{tabular}




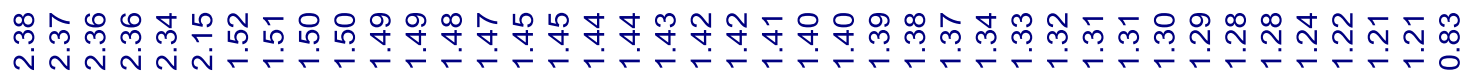

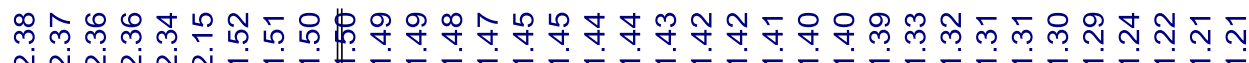

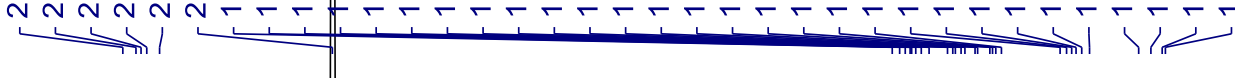
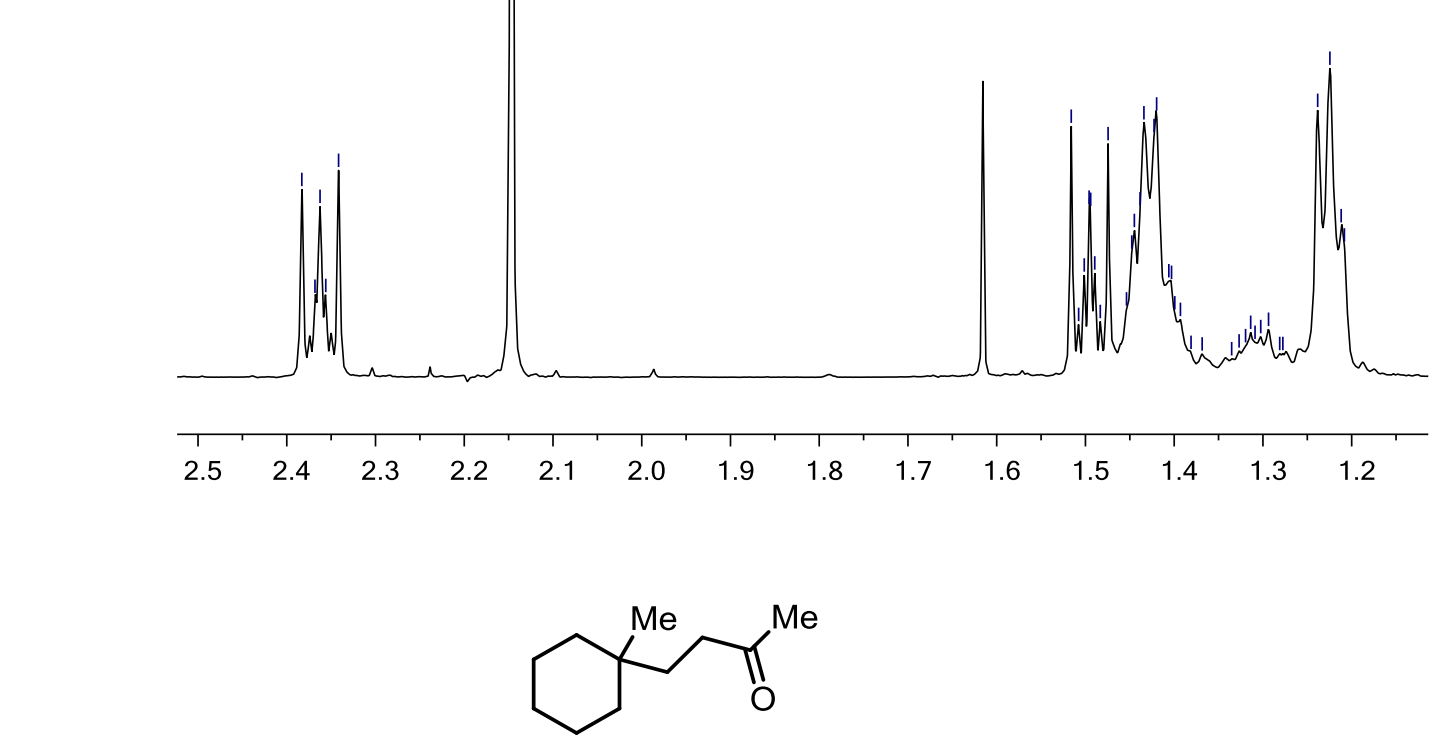

\section{.}




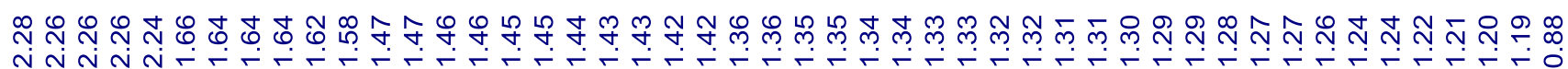

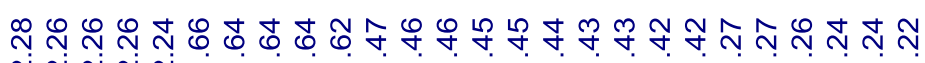

管
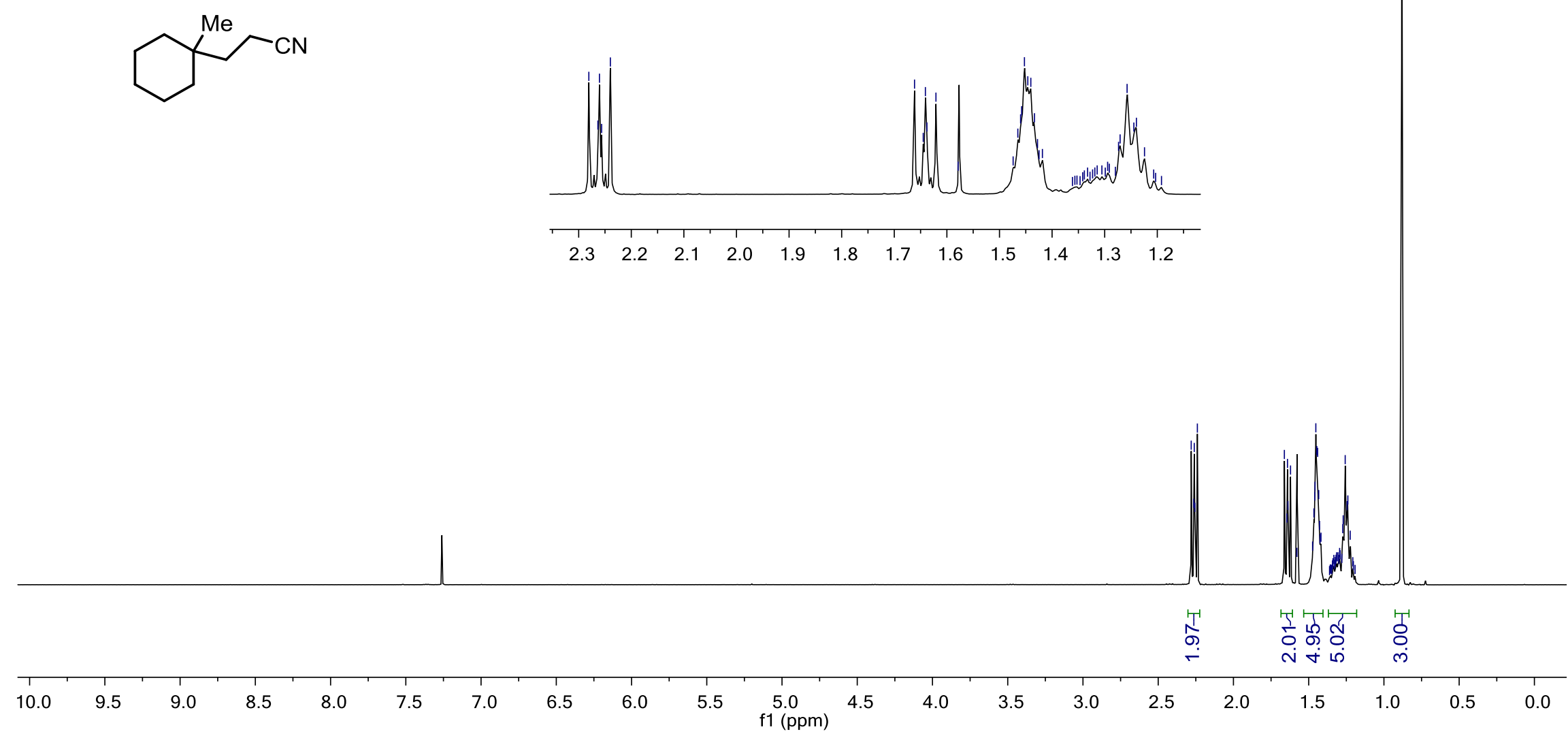

SI-109 
${ }^{13} \mathrm{C}-\mathrm{NMR}\left(101 \mathrm{MHz}, \mathrm{CDCl}_{3}\right)$ for compound $4 \mathrm{f}$

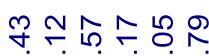

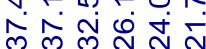
$1<1$

$\stackrel{?}{\stackrel{r}{r}}$

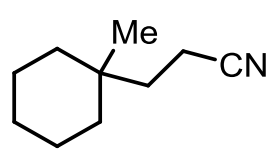

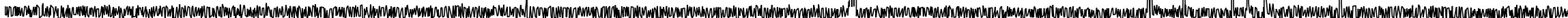

$90 \quad 180$ $170 \quad 160$
$150 \quad 140$ 


\section{${ }^{1} \mathrm{H}-\mathrm{NMR}\left(400 \mathrm{MHz}, \mathrm{CDCl}_{3}\right)$ for compound $\mathbf{4 g}$}

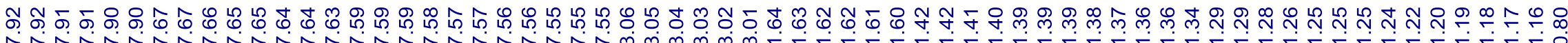

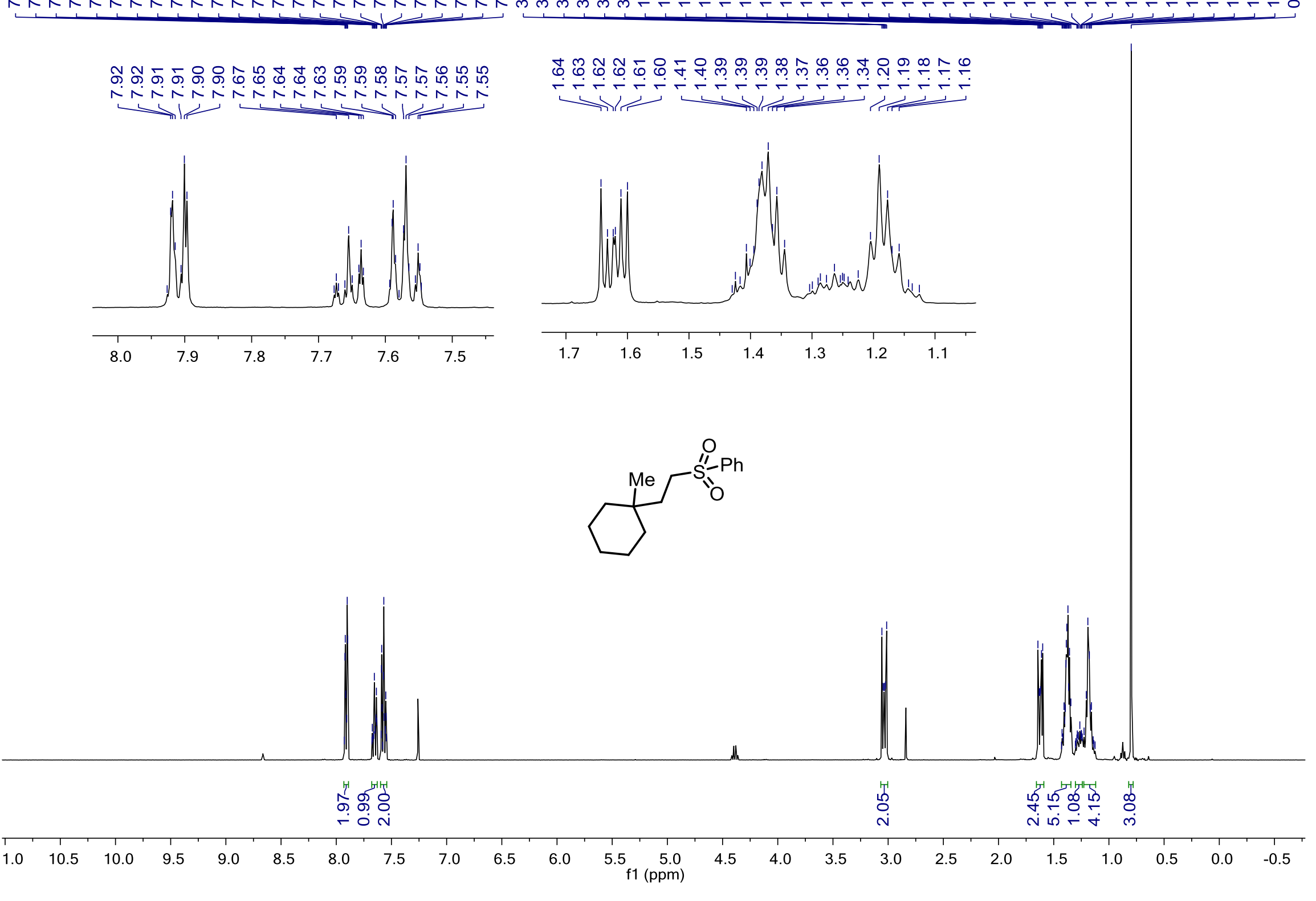

SI-111 
${ }^{13} \mathrm{C}-\mathrm{NMR}(101 \mathrm{MHz}, \mathrm{CDCl} / 3)$ for compound $\mathbf{4 g}$

livi

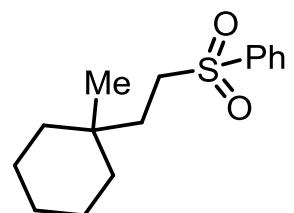




\section{${ }^{1} \mathrm{H}-\mathrm{NMR}\left(400 \mathrm{MHz}, \mathrm{CDCl}_{3}\right)$ for compound $4 \mathrm{~h}$}

○ ه

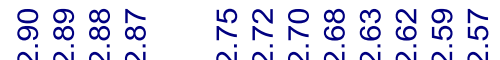

viju

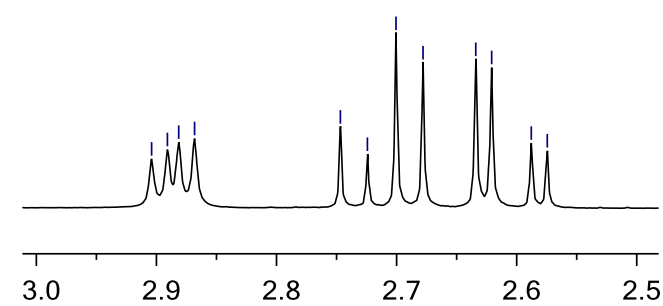

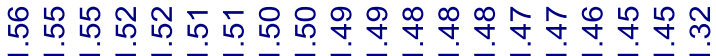

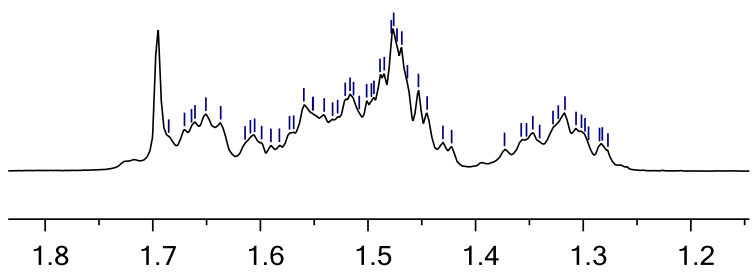

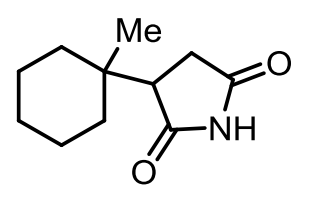

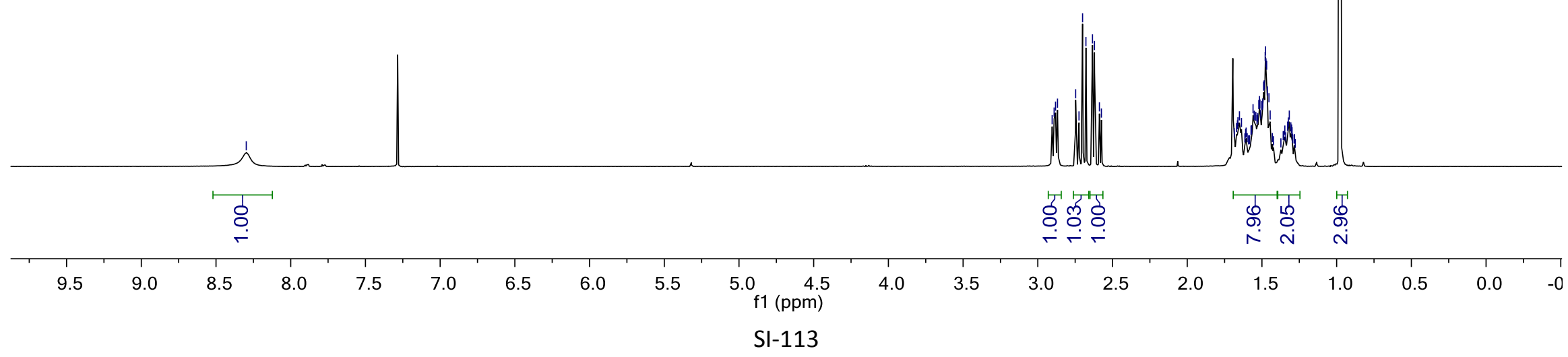


${ }^{13} \mathrm{C}-\mathrm{NMR}(101 \mathrm{MHz}, \mathrm{CDCl} / 3)$ for compound $\mathbf{4 h}$

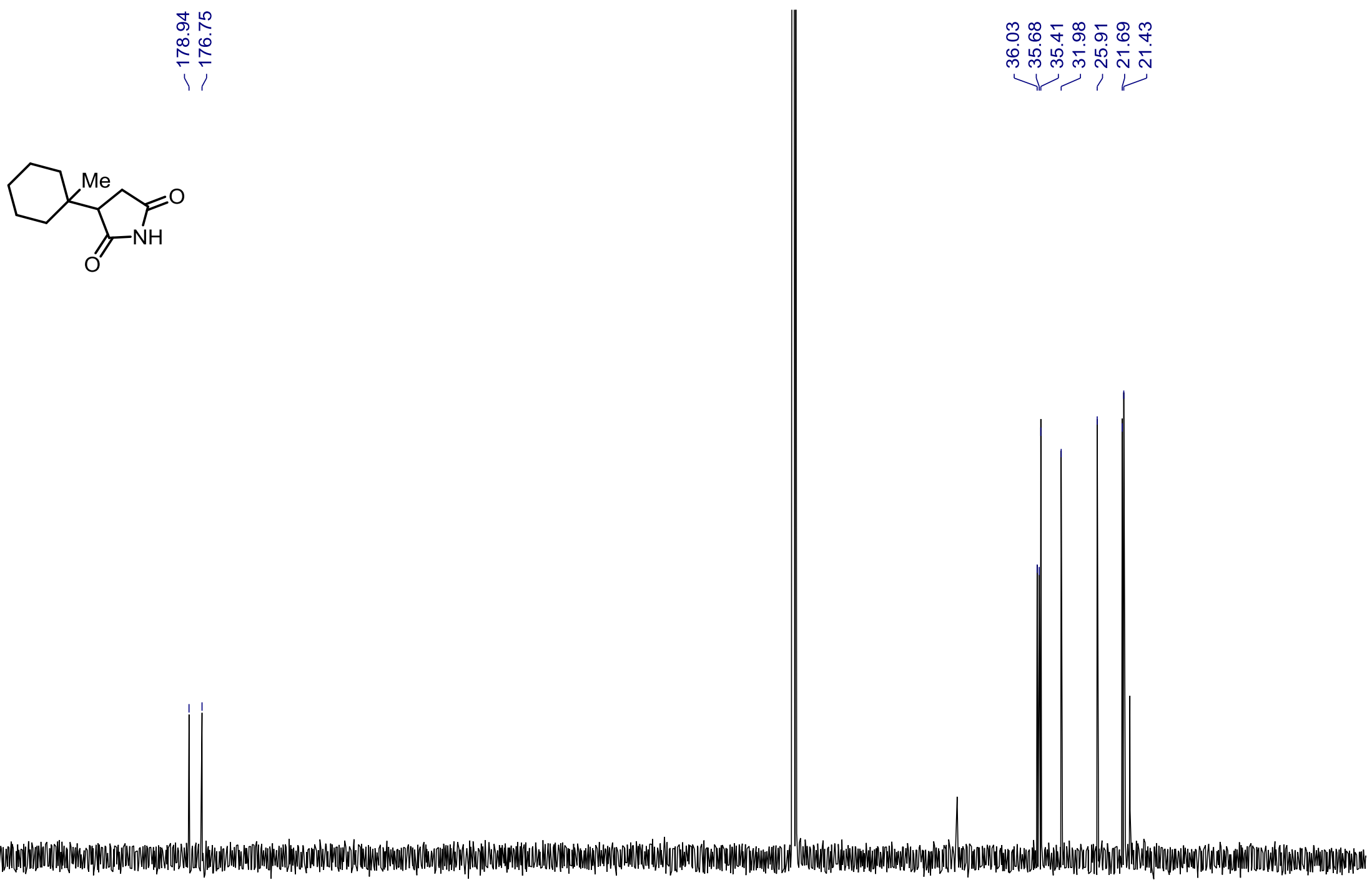

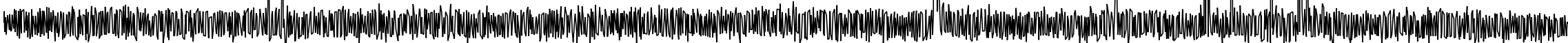

$\begin{array}{llllllllllll}210 & 200 & 190 & 180 & 170 & 160 & 150 & 140 & 130 & 120 & 110 & 100\end{array}$

80

60

$50 \quad 40$

20

$\begin{array}{lll}10 & 0 & -10\end{array}$

SI-114 
${ }^{1} \mathrm{H}-\mathrm{NMR}\left(400 \mathrm{MHz}, \mathrm{CDCl}_{3}\right)$ for compound 4i

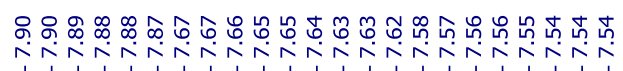

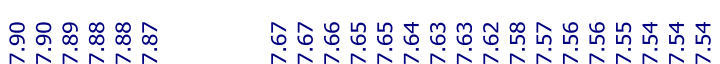

N

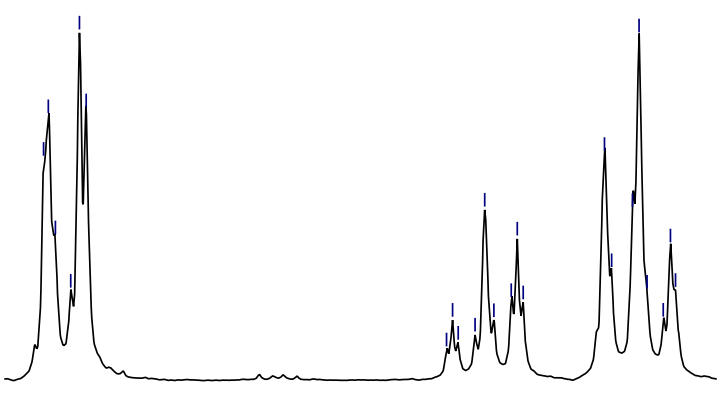

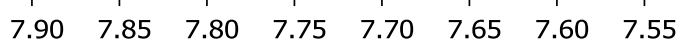

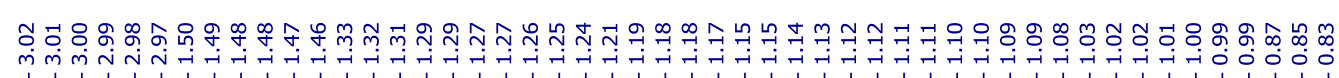

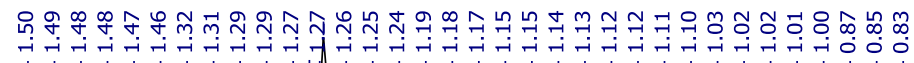
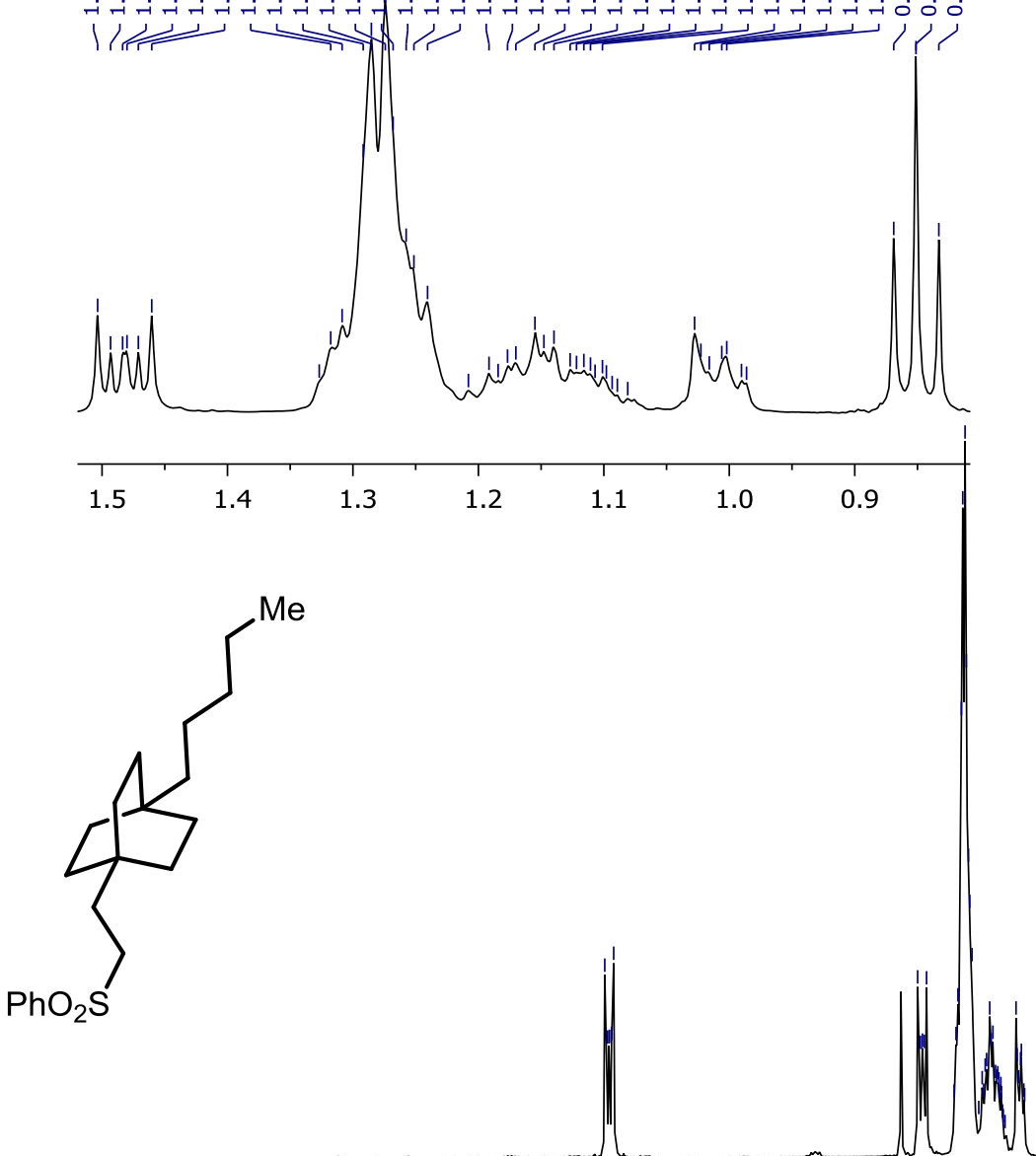

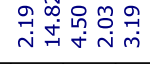


${ }^{13} \mathrm{C}-\mathrm{NMR}\left(101 \mathrm{MHz}, \mathrm{CDCl}_{3}\right)$ for compound 4i

范

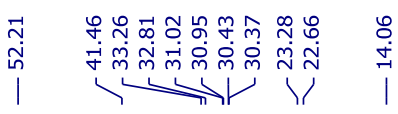

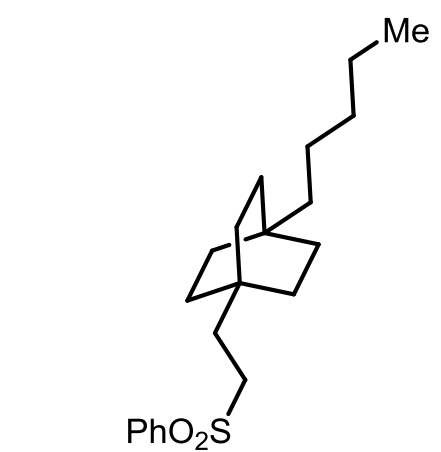

促

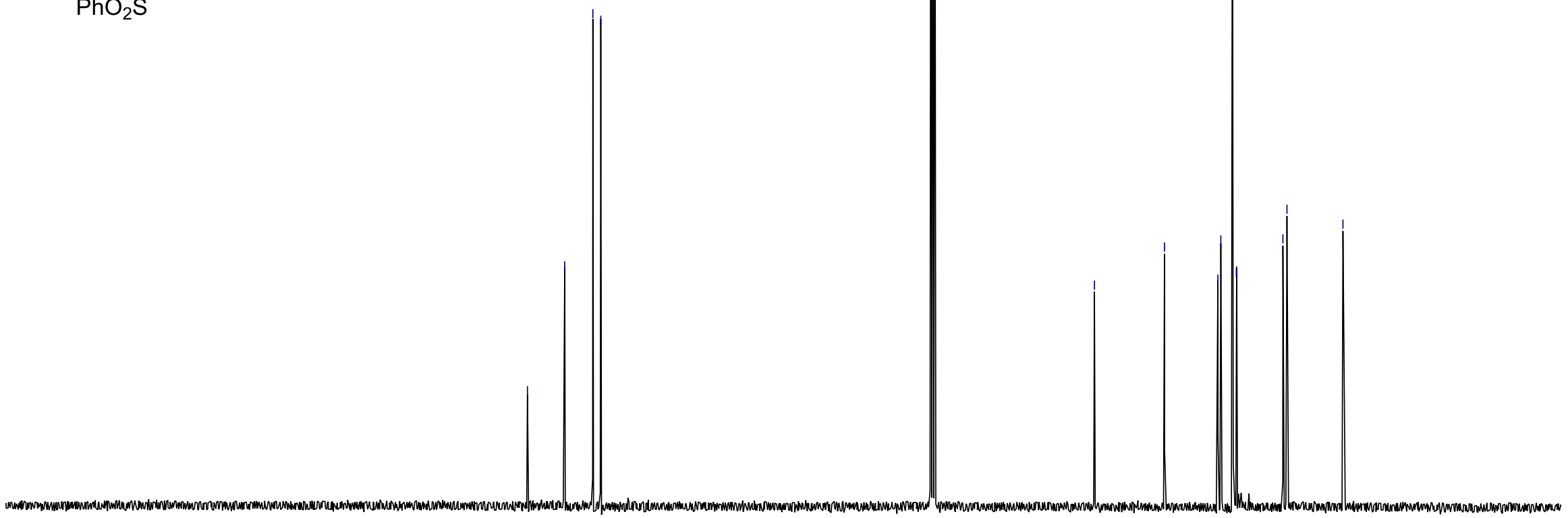


${ }^{1} \mathrm{H}-\mathrm{NMR}\left(400 \mathrm{MHz}, \mathrm{CDCl}_{3}\right)$ for compound $4 \mathrm{j}$

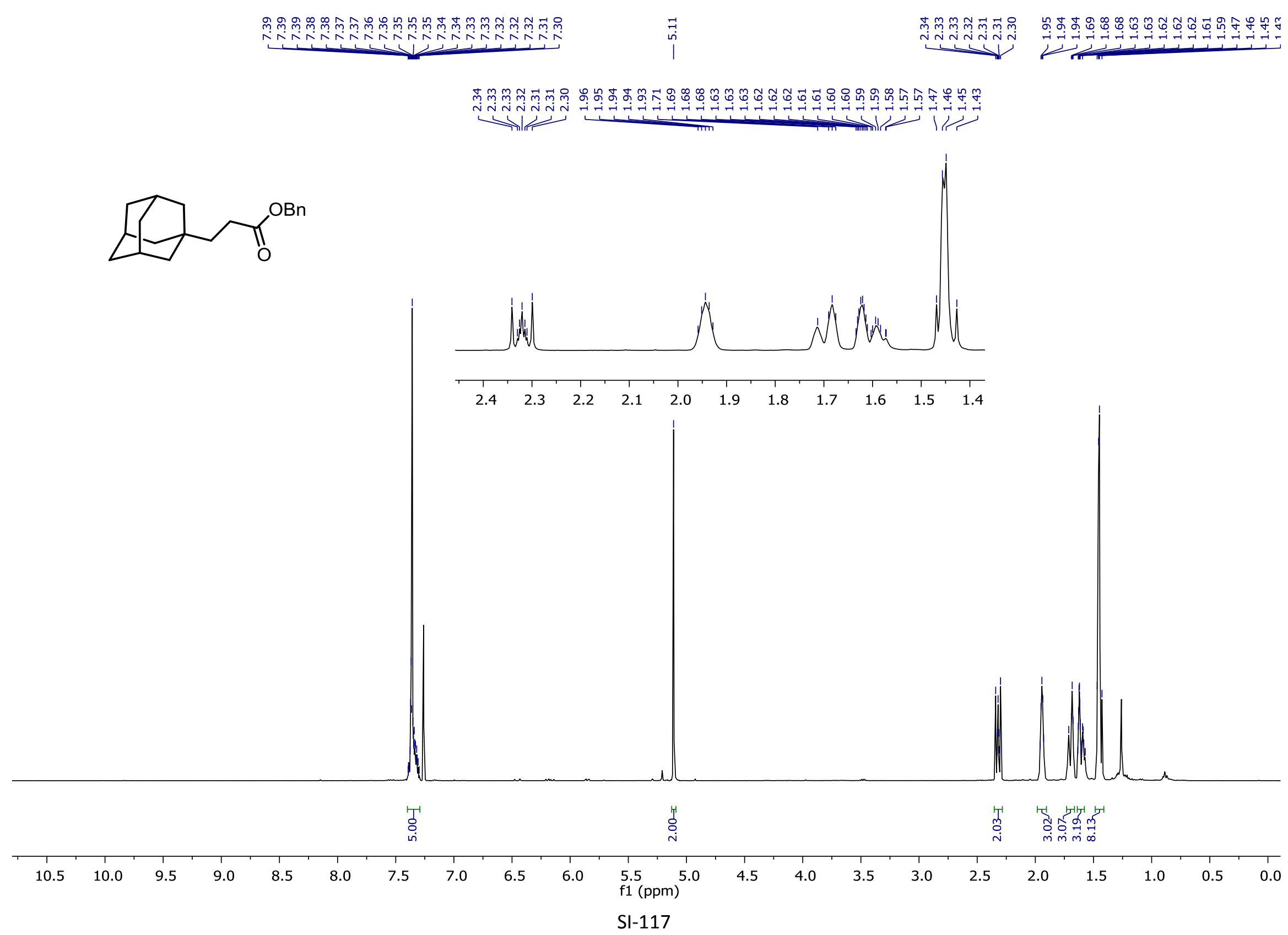


${ }^{13} \mathrm{C}-\mathrm{NMR}\left(101 \mathrm{MHz}, \mathrm{CDCl}_{3}\right)$ for compound $\mathbf{4 j}$

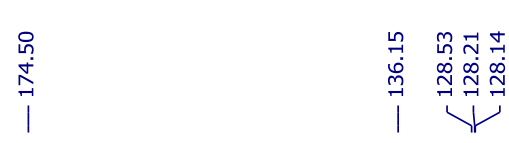

苟

अर

$1114 \mathrm{k}$
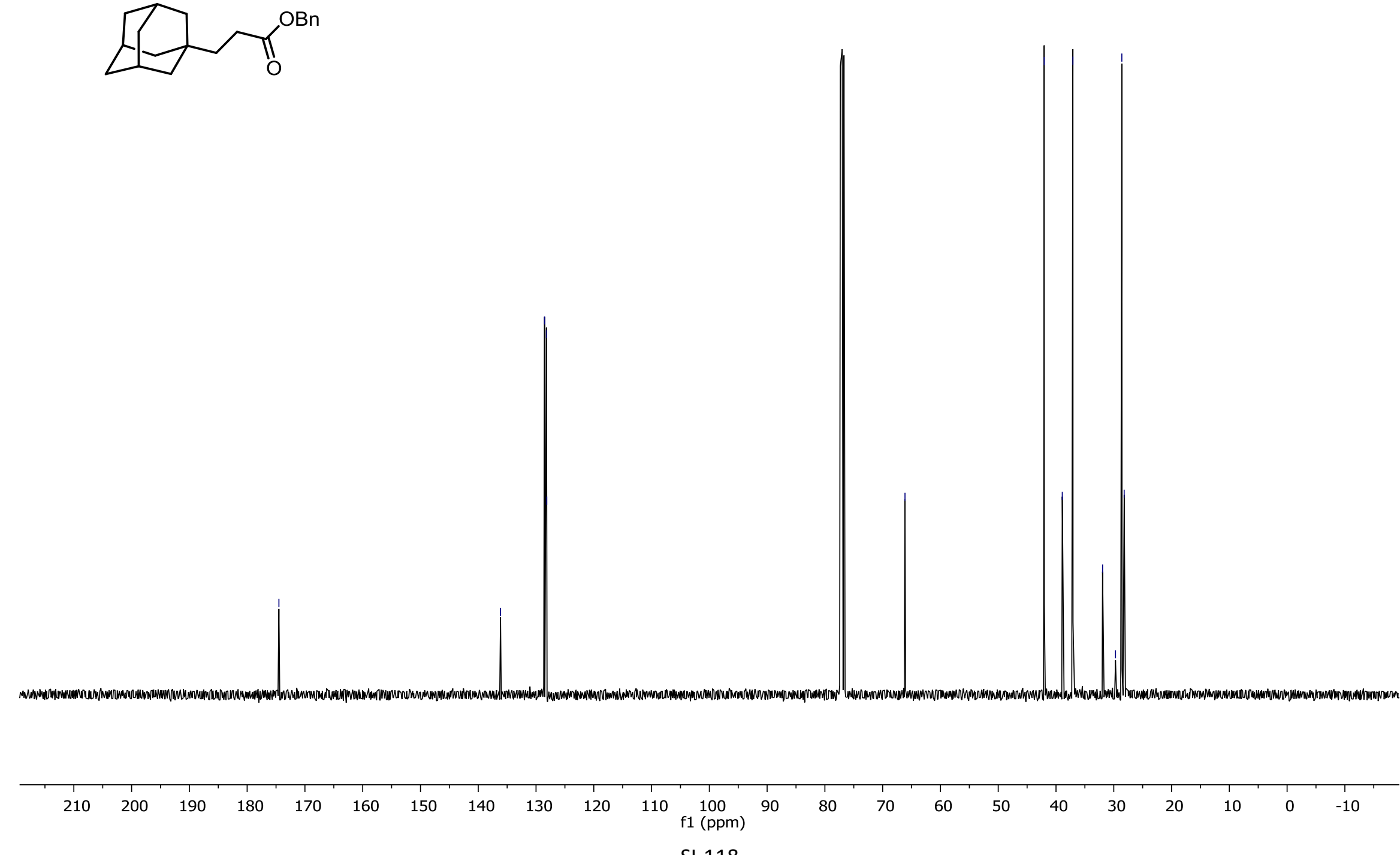

SI-118 
${ }^{1} \mathrm{H}-\mathrm{NMR}\left(400 \mathrm{MHz}, \mathrm{CDCl}_{3}\right)$ for compound $\mathbf{4 k}$

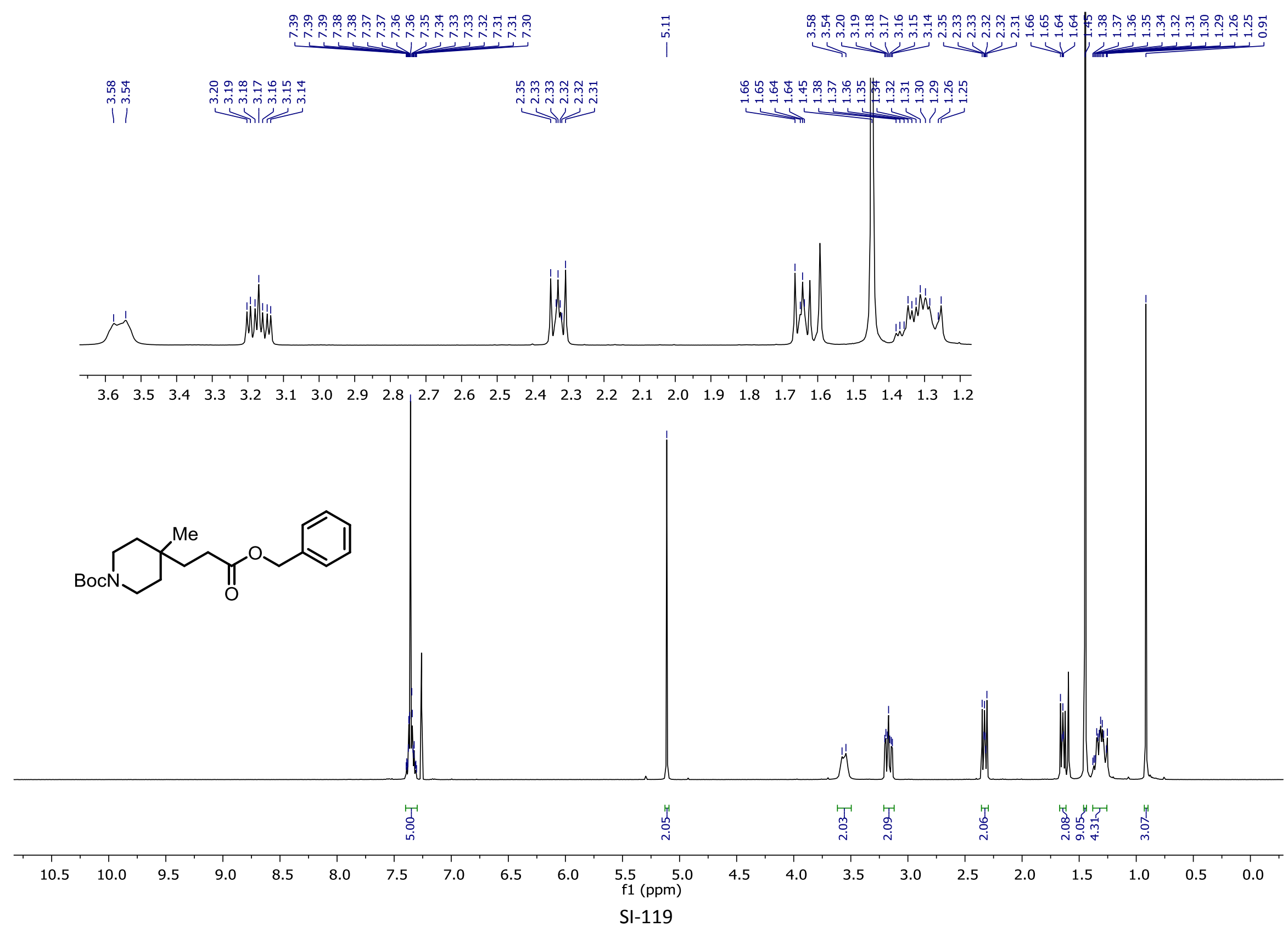


${ }^{13} \mathrm{C}-\mathrm{NMR}(101 \mathrm{MHz}, \mathrm{CDCl} / 3)$ for compound $\mathbf{4 k}$

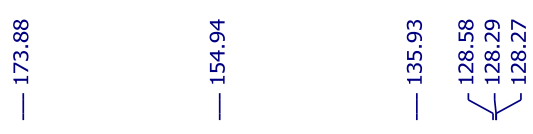<smiles>CC1(CCC(=O)OCc2ccccc2)CCN(C(=O)OC(C)(C)C)CC1</smiles>

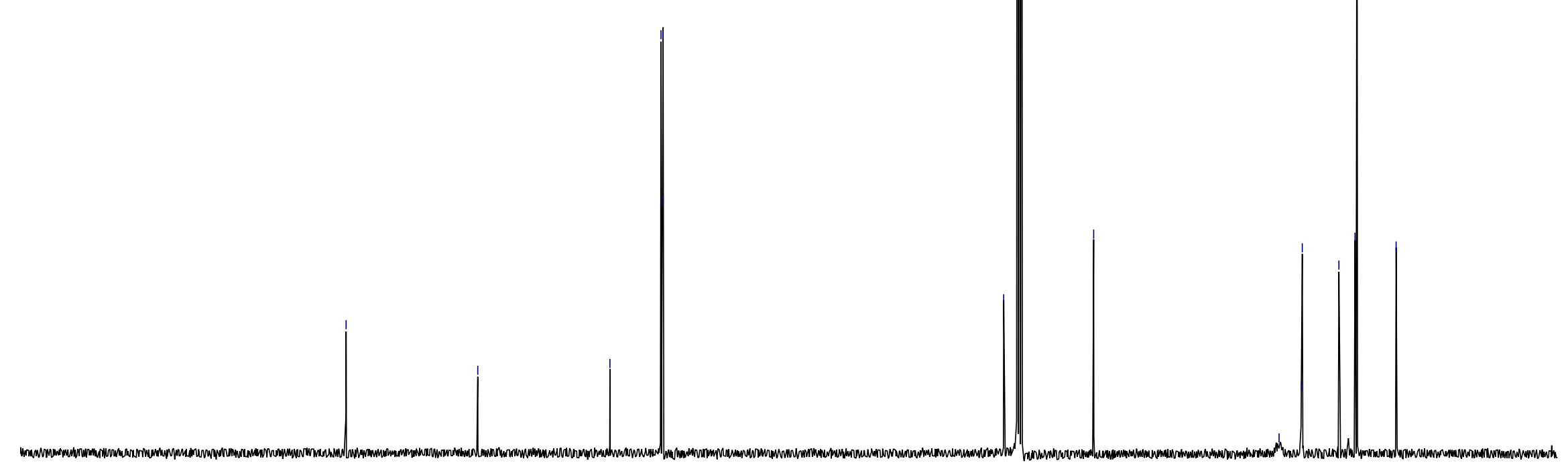

$$
\text { :20 }
$$

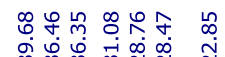

$|V| Y \mid$ 


\section{资}
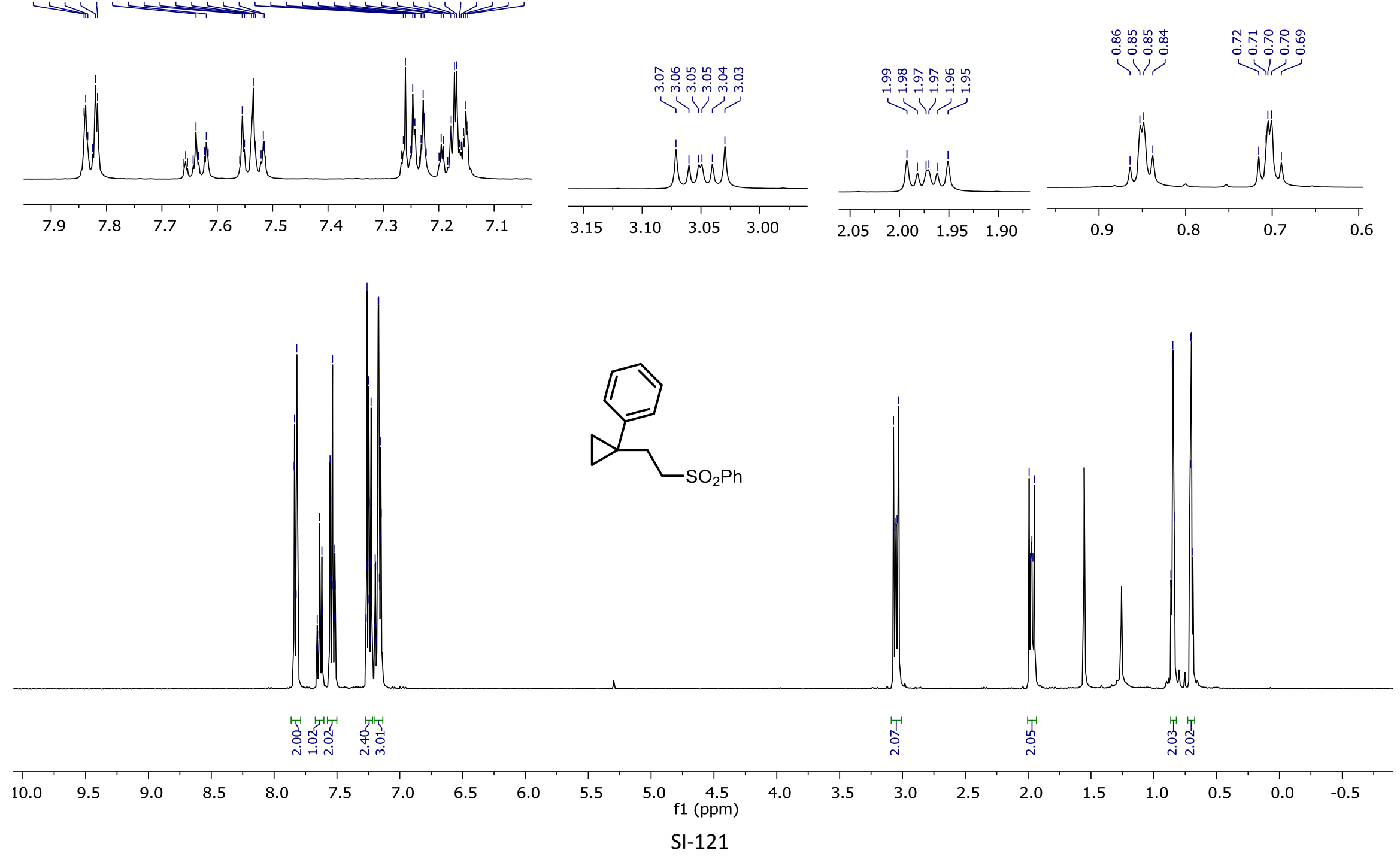
${ }^{13} \mathrm{C}-\mathrm{NMR}\left(101 \mathrm{MHz}, \mathrm{CDCl}_{3}\right)$ for compound 4I

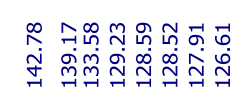

1 | 111

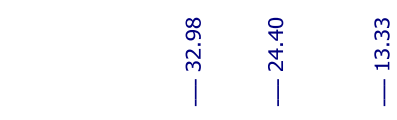

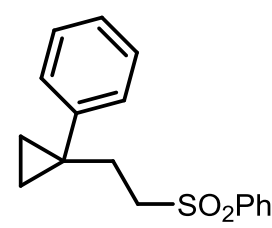

\begin{tabular}{|c|c|c|c|c|c|c|c|c|c|c|c|c|c|c|c|c|c|c|c|c|c|c|c|}
\hline 210 & 20 & & 190 & 180 & 170 & 160 & 150 & 140 & 130 & 120 & 110 & $\begin{array}{c}100 \\
\mathrm{f} 1(\mathrm{ppm})\end{array}$ & 90 & 80 & 70 & 60 & 50 & 40 & 30 & 20 & 10 & 0 & -10 \\
\hline & & & & & & & & & & & & SI-122 & & & & & & & & & & & \\
\hline
\end{tabular}

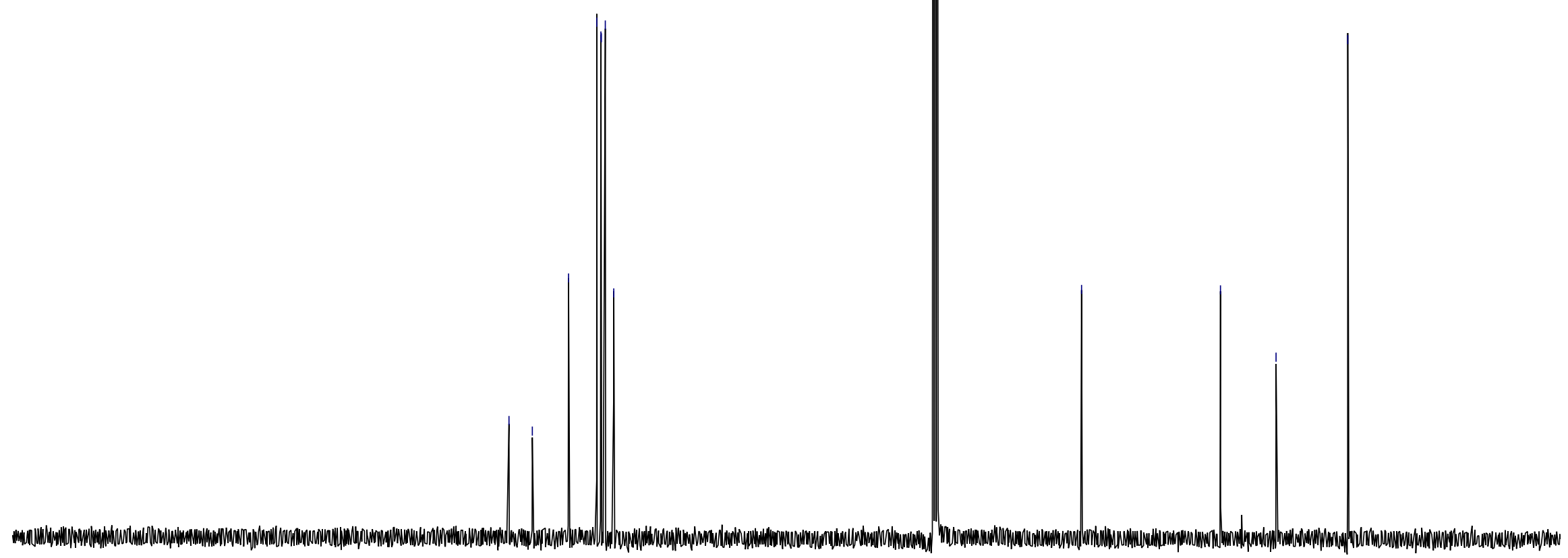


${ }^{1} \mathrm{H}-\mathrm{NMR}\left(400 \mathrm{MHz}, \mathrm{CDCl}_{3}\right.$ ) for compound $\mathbf{4 m}$

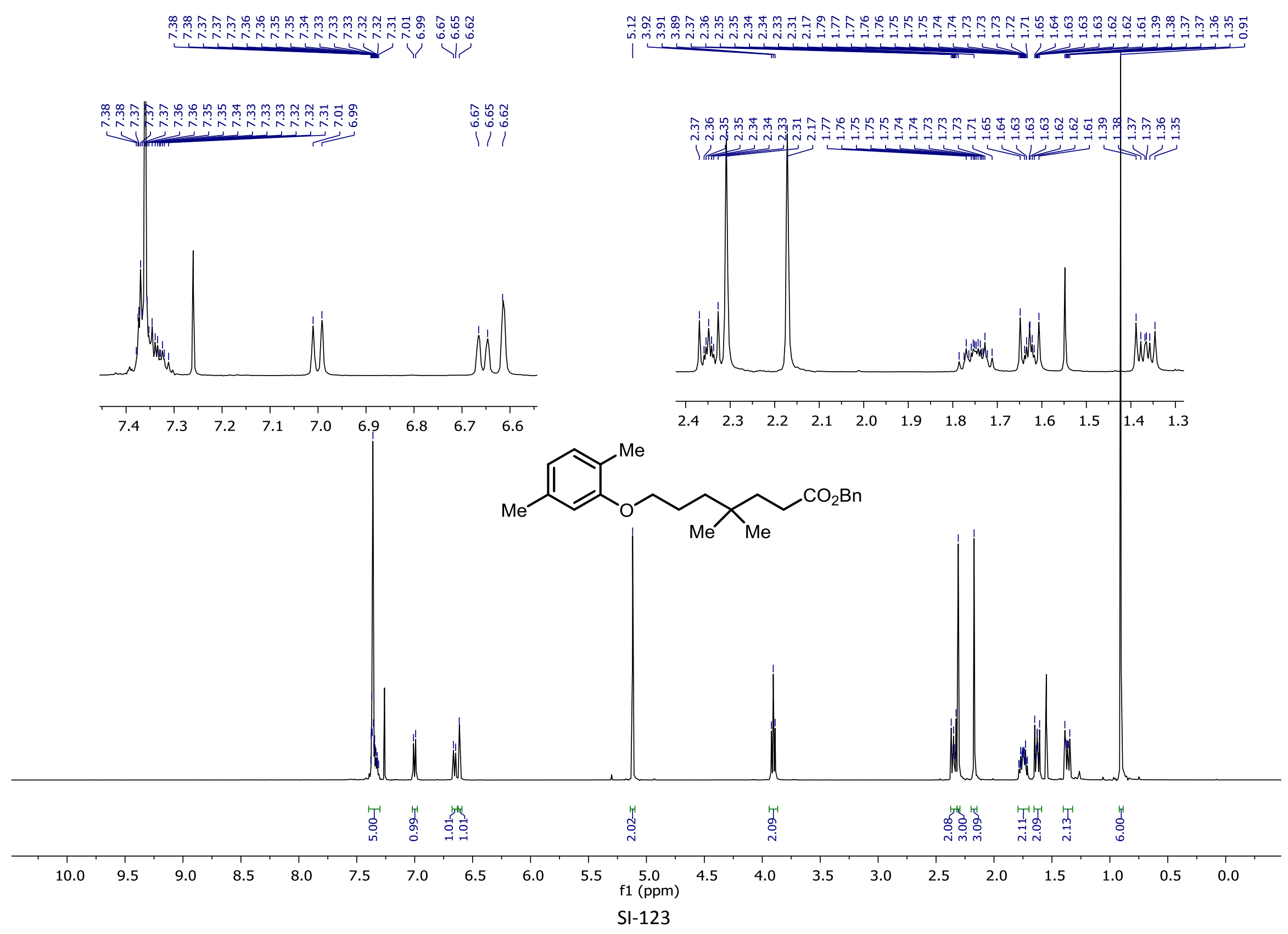


${ }^{13} \mathrm{C}-\mathrm{NMR}(101 \mathrm{MHz}, \mathrm{CDCl} / 3)$ for compound $\mathbf{4 m}$

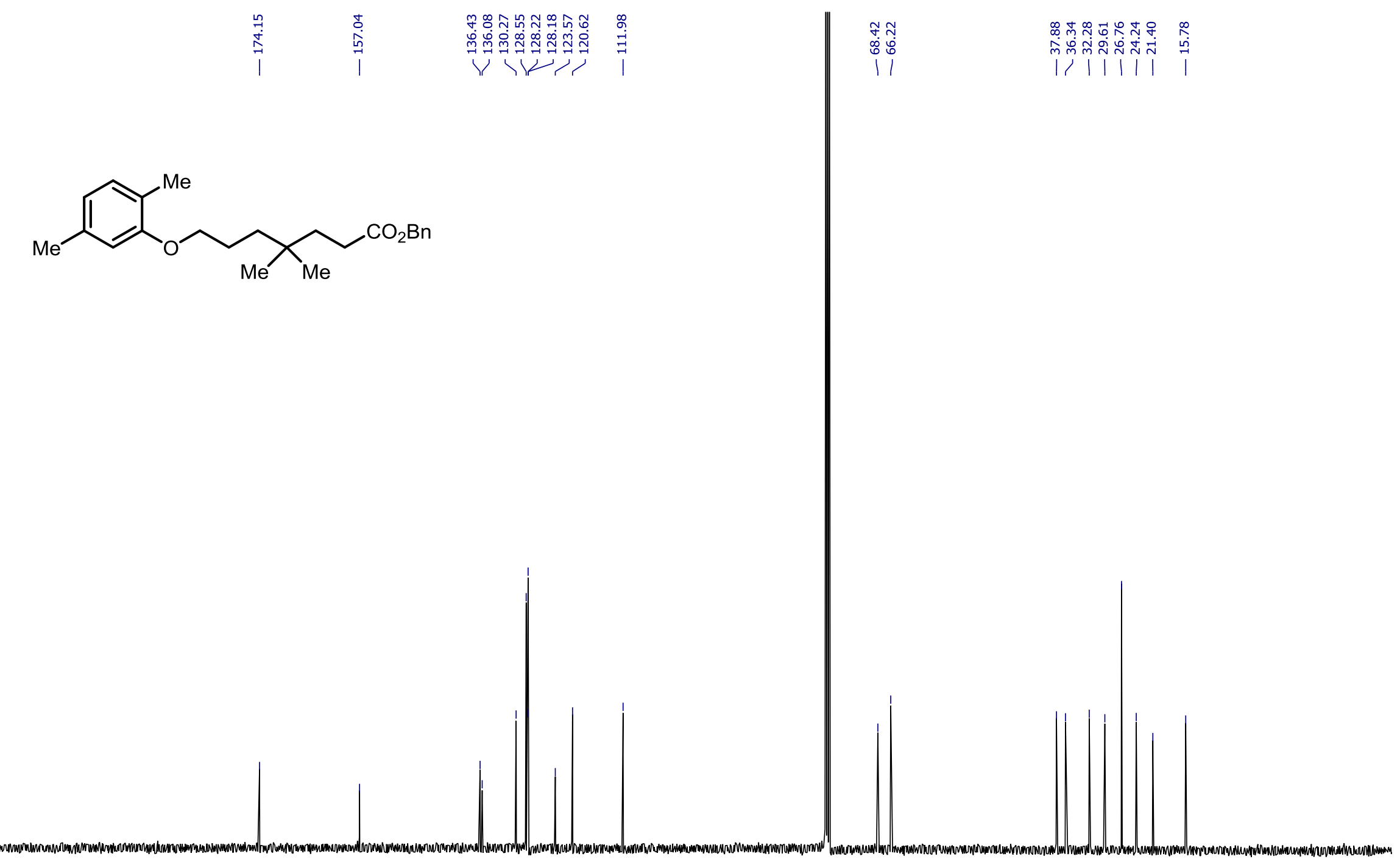

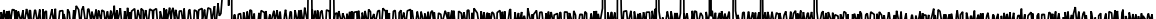

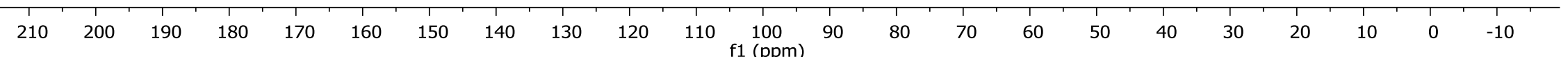

SI-124 


\section{${ }^{1} \mathrm{H}-\mathrm{NMR}\left(400 \mathrm{MHz}, \mathrm{CDCl}_{3}\right)$ for compound $4 \mathrm{n}$}

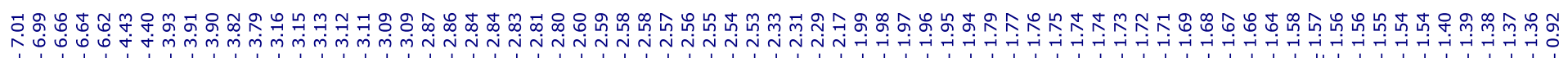

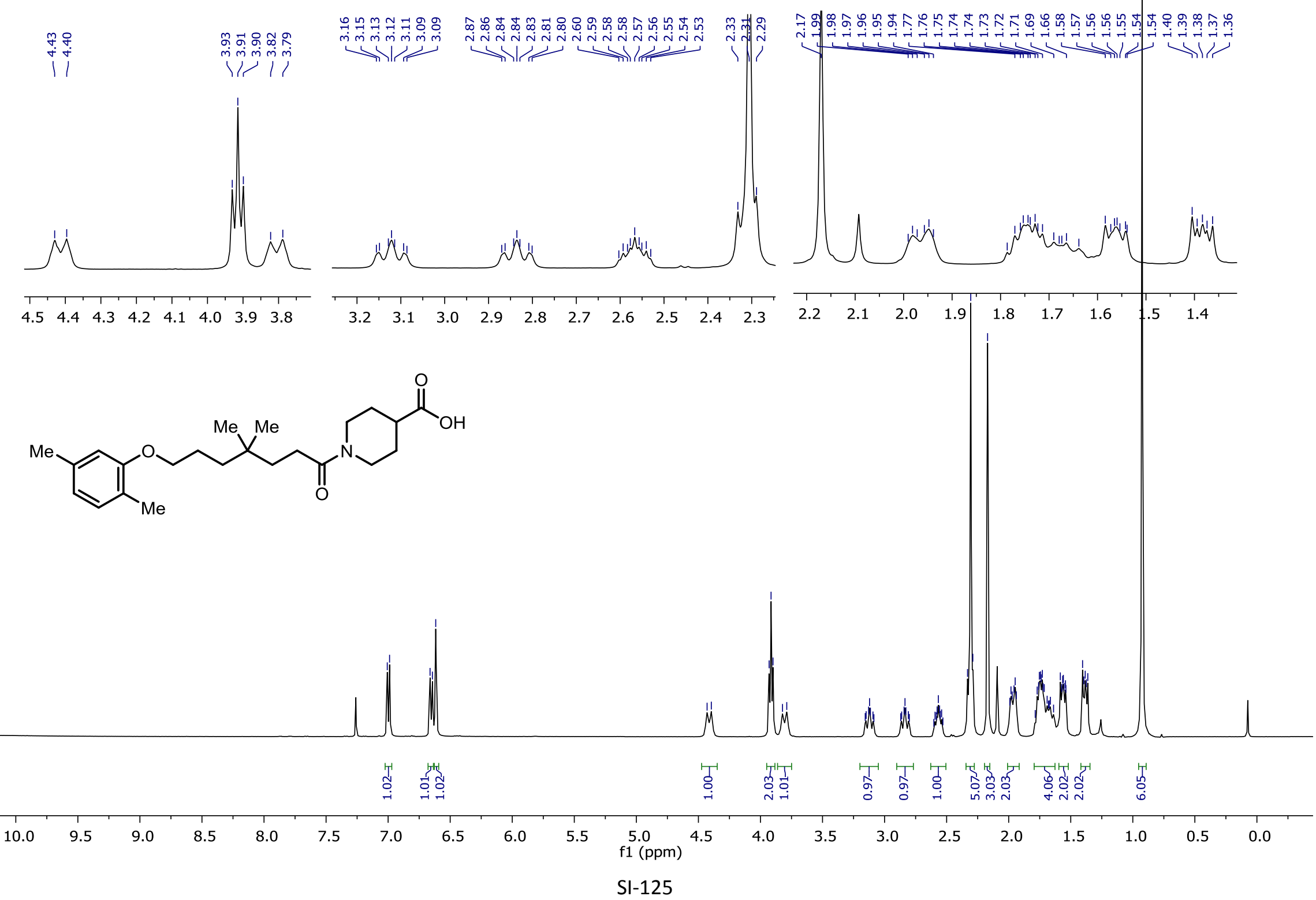


${ }^{13} \mathrm{C}-\mathrm{NMR}\left(101 \mathrm{MHz}, \mathrm{CDCl}_{3}\right)$ for compound $4 n$

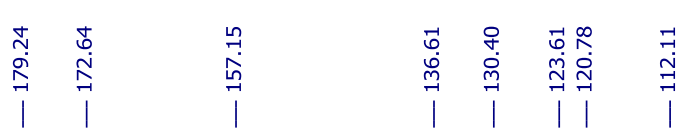

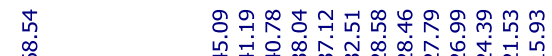

每

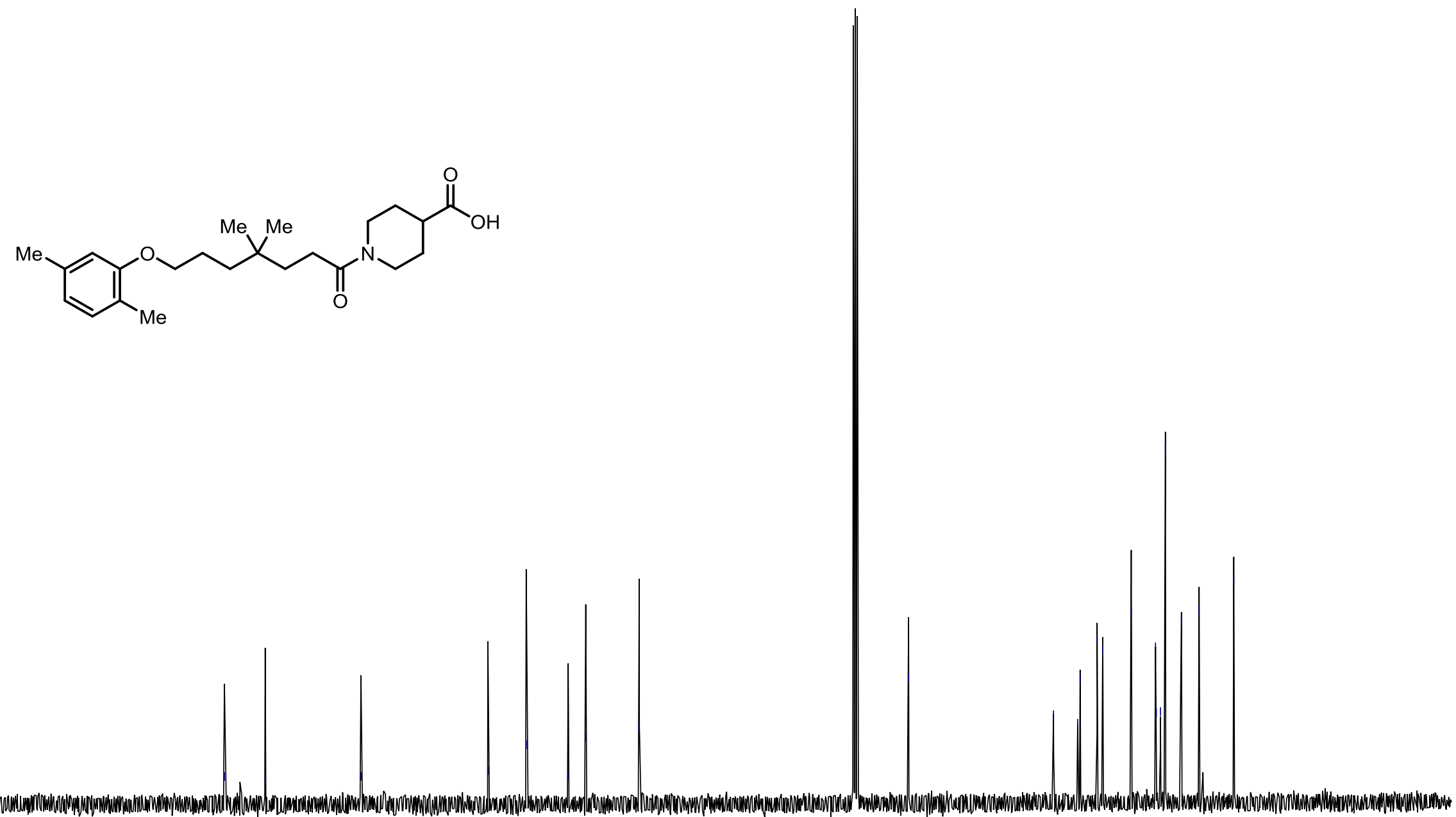

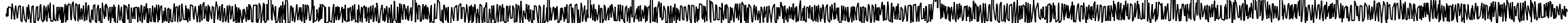




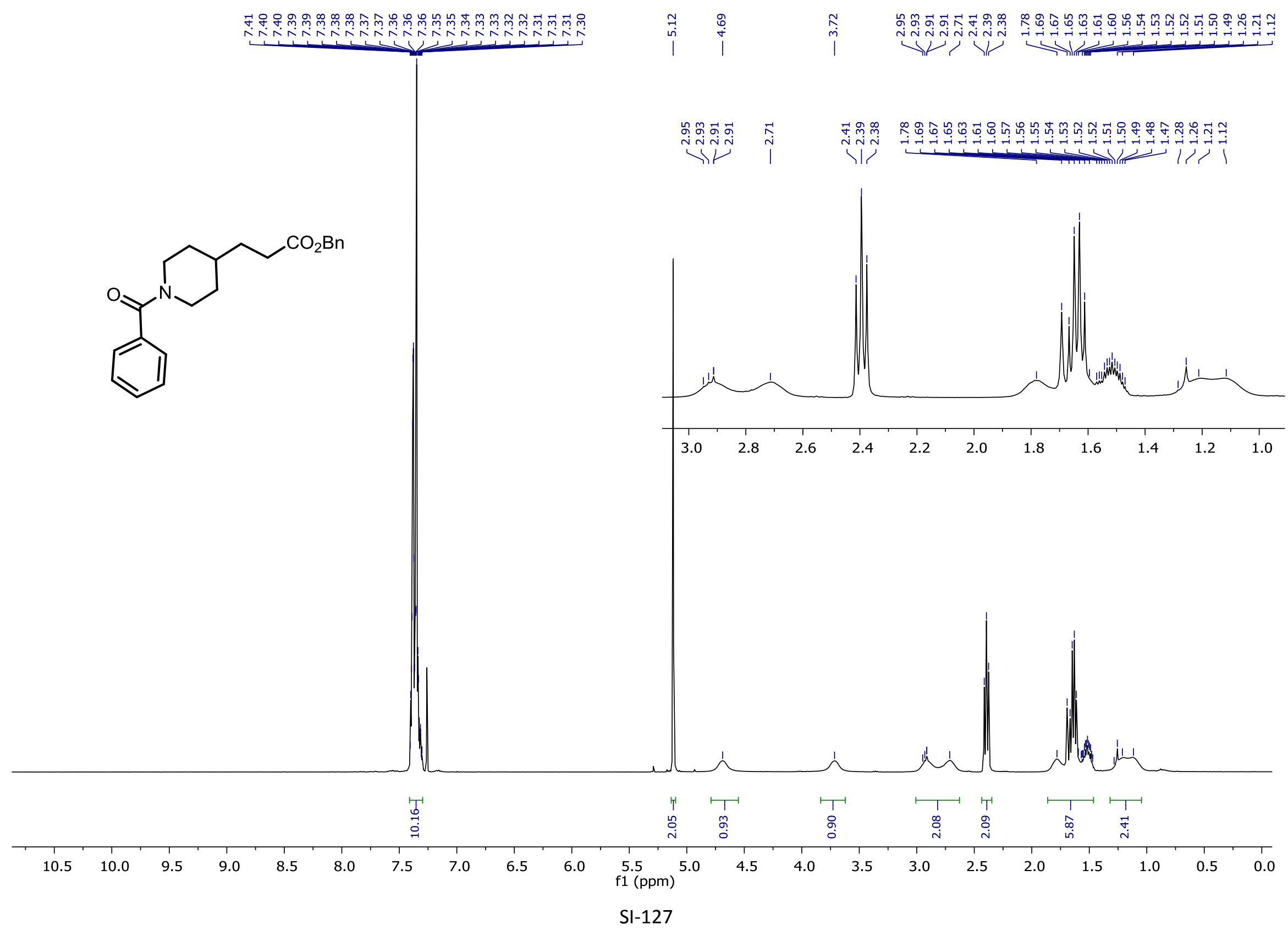


${ }^{13} \mathrm{C}-\mathrm{NMR}(101 \mathrm{MHz}, \mathrm{CDCl} / 3)$ for compound 40

\begin{tabular}{|c|c|c|c|}
\hline 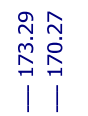 & 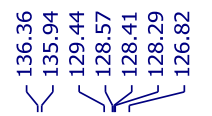 & $\infty$ & \\
\hline
\end{tabular}
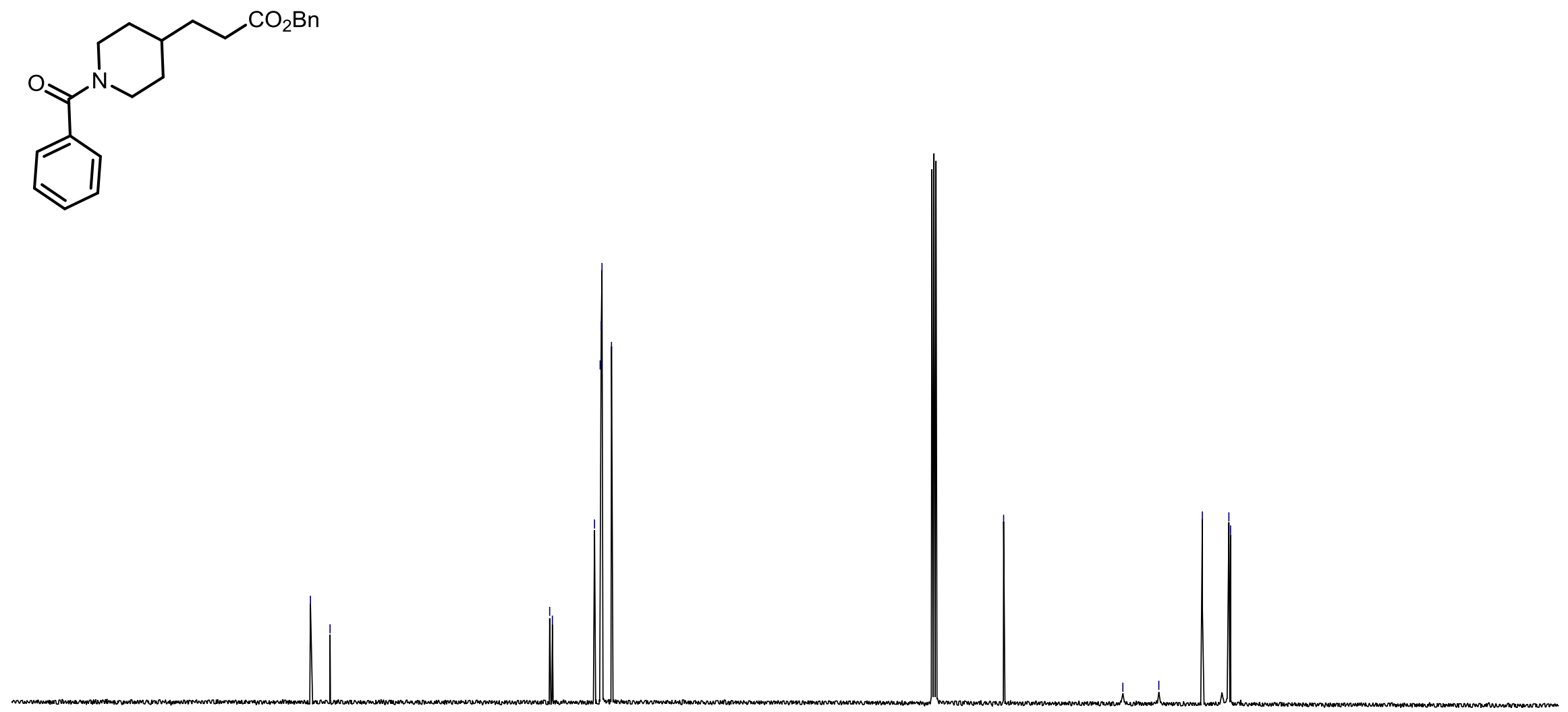

$210 \quad 200$

160

$150 \quad 140$

$130 \quad 120 \quad 110 \begin{gathered}100 \\ \mathrm{f} 1(\mathrm{ppm})\end{gathered}$

80

$70 \quad 60$

50

40

SI-128 


\section{${ }^{1} \mathrm{H}-\mathrm{NMR}\left(400 \mathrm{MHz}, \mathrm{CDCl}_{3}\right)$ for compound $4 p$}

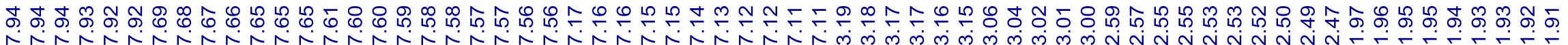

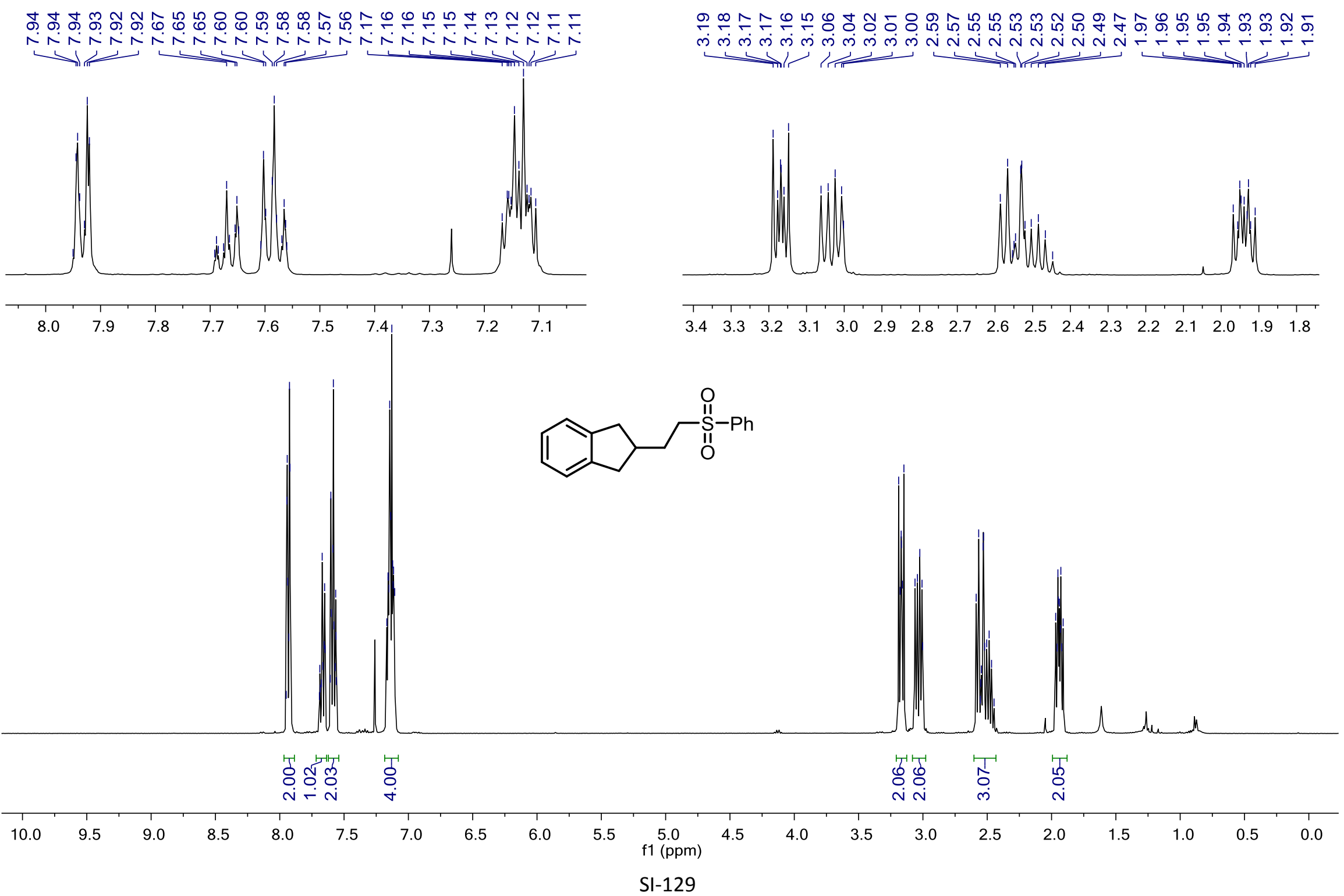


${ }^{13} \mathrm{C}-\mathrm{NMR}\left(101 \mathrm{MHz}, \mathrm{CDCl}_{3}\right)$ for compound $4 p$

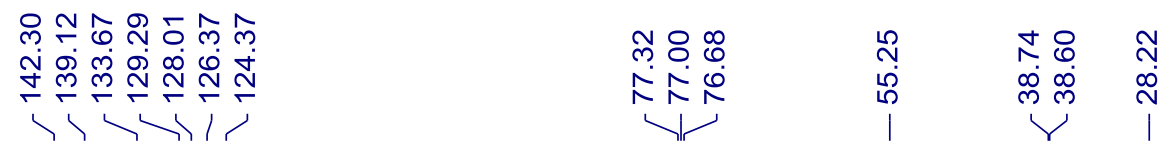
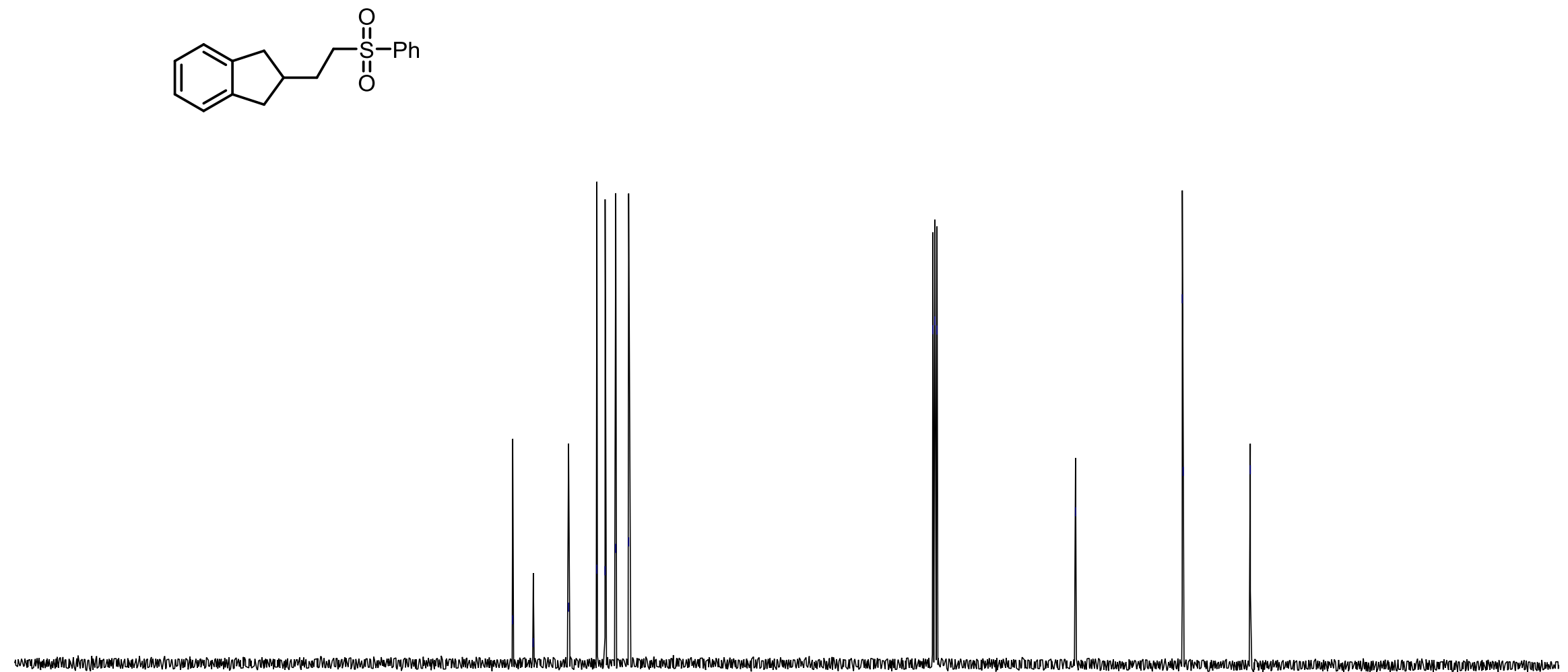

\begin{tabular}{|c|c|c|c|c|c|c|c|c|c|c|c|c|c|c|c|c|c|c|c|c|c|c|}
\hline 210 & 200 & 190 & 180 & 170 & 160 & 150 & 140 & 130 & 120 & 110 & $\begin{array}{c}100 \\
\mathrm{f} 1(\mathrm{ppm})\end{array}$ & 90 & 80 & 70 & 60 & 50 & 40 & 30 & 20 & 10 & 0 & -10 \\
\hline & & & & & & & & & & & SI-130 & & & & & & & & & & & \\
\hline
\end{tabular}




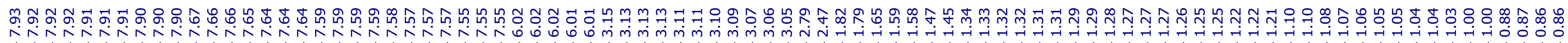
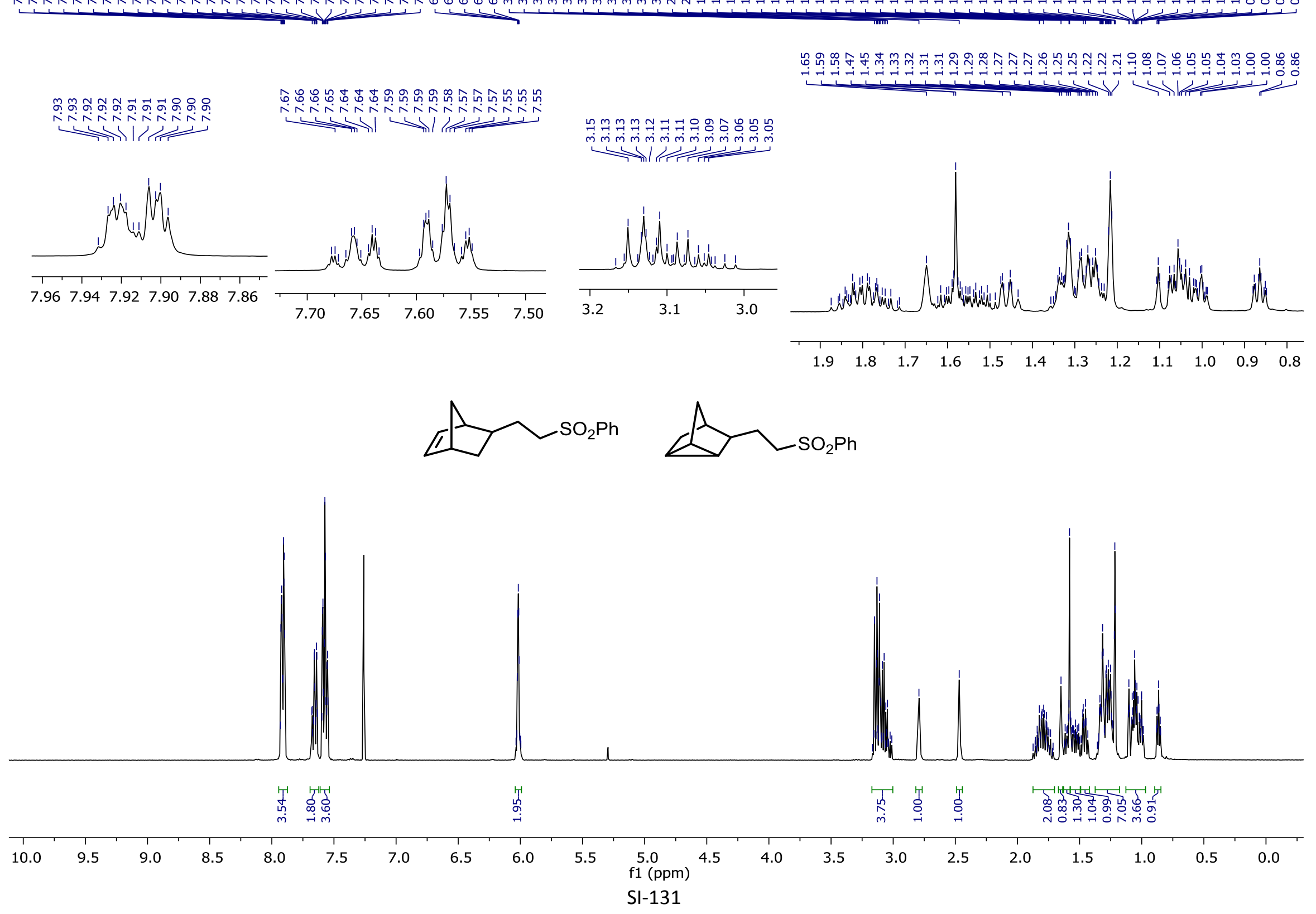
${ }^{13} \mathrm{C}-\mathrm{NMR}\left(101 \mathrm{MHz}, \mathrm{CDCl}_{3}\right)$ for compound $\mathbf{4 q}$ and $\mathbf{4 q} \mathbf{q}^{\prime}$

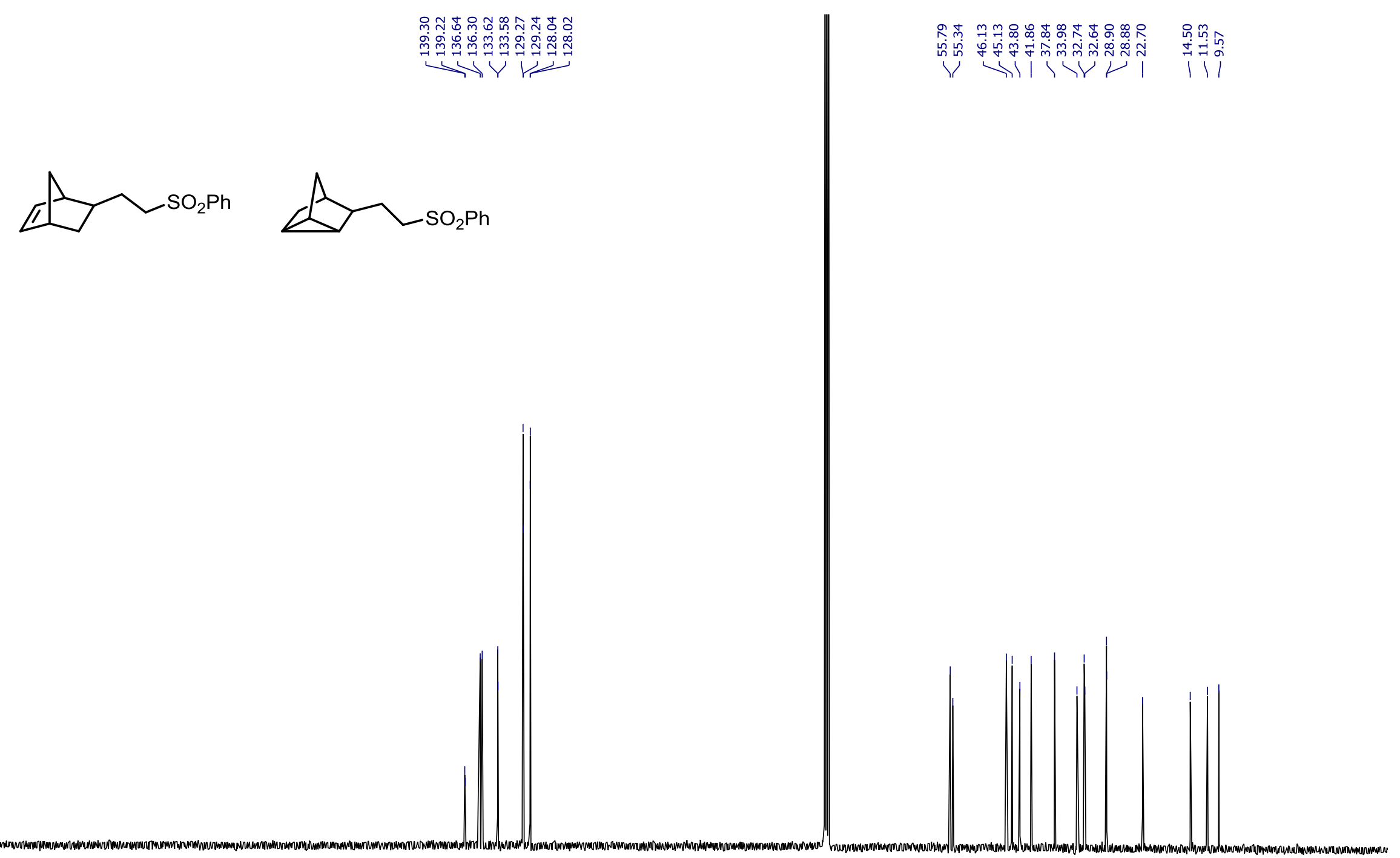

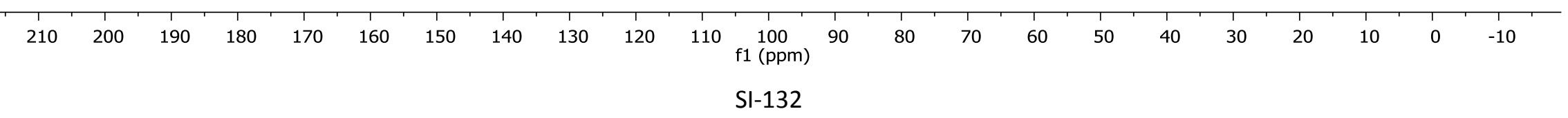


DEPT $135 \mathrm{NMR}\left(101 \mathrm{MHz}, \mathrm{CDCl}_{3}\right)$ for compound $\mathbf{4 q}$ and $\mathbf{4 q ^ { \prime }}$
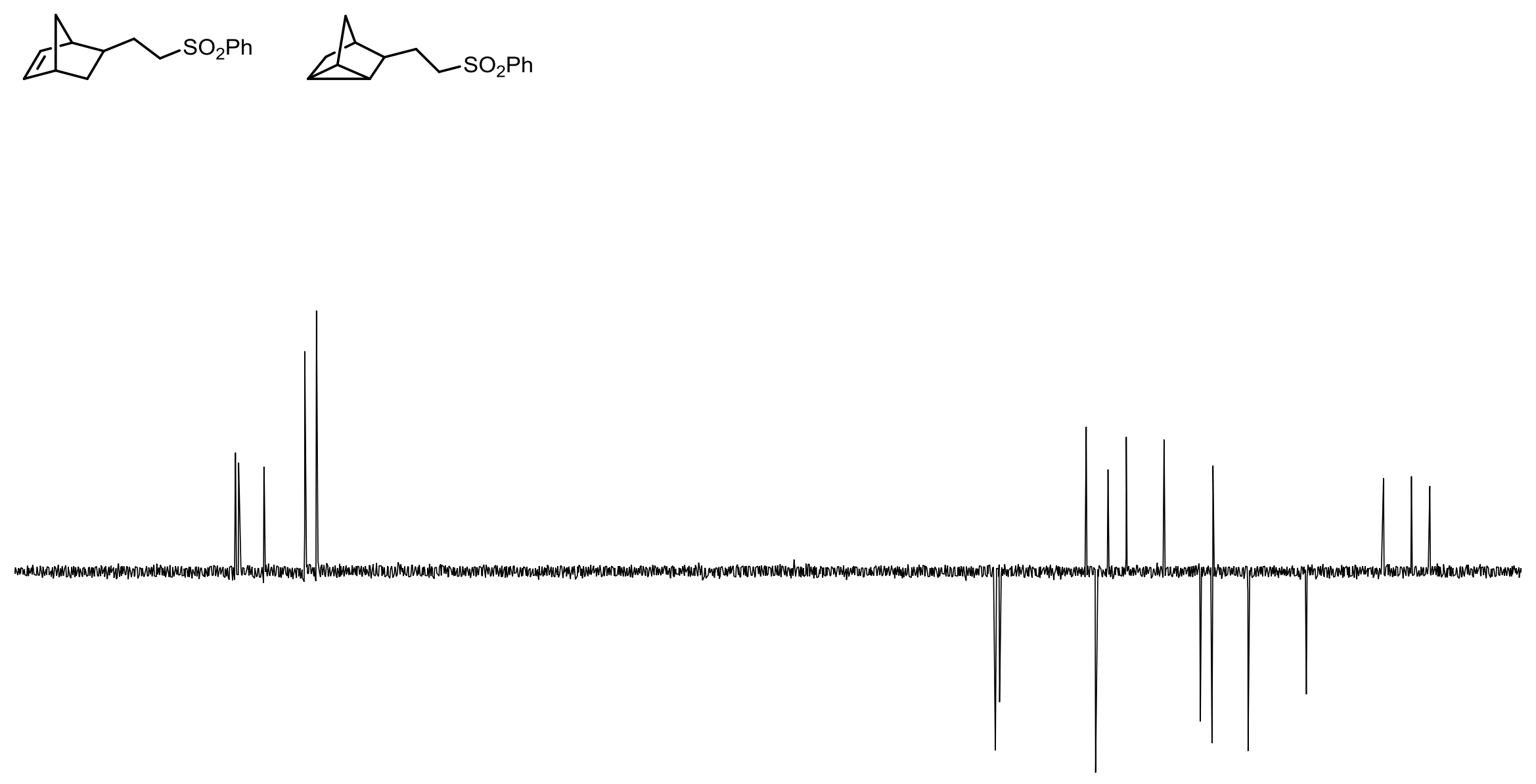

60

$150 \quad 140$

130

120

110

100

90

f1 $\stackrel{80}{(\mathrm{ppm})}$

70

60

50

40

30

20

10

SI-133 
$\operatorname{COSY} \mathrm{NMR}\left(400 \mathrm{MHz}, \mathrm{CDCl}_{3}\right)$ for compound $\mathbf{4 q}$ and $\mathbf{4 \boldsymbol { q } ^ { \prime }}$

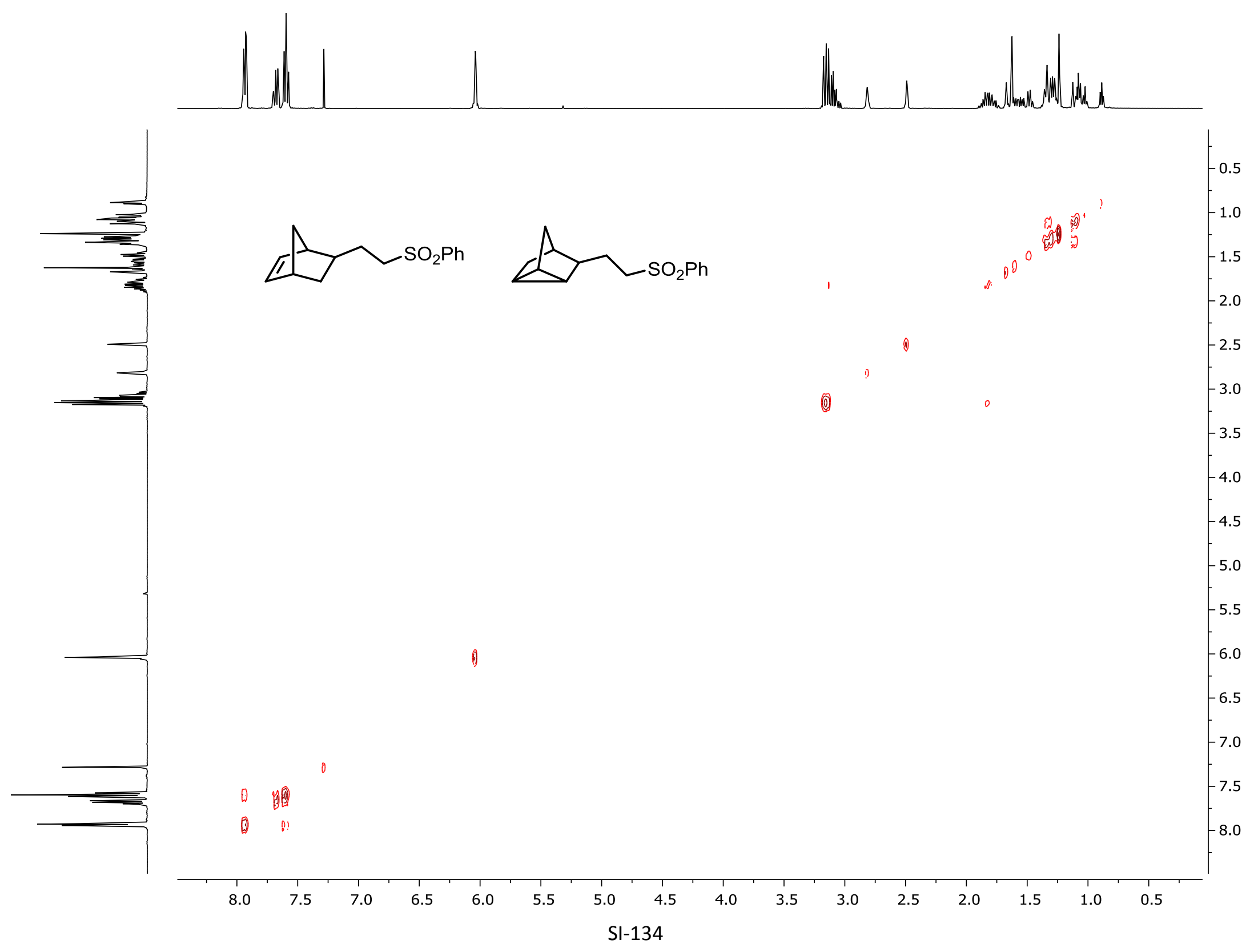


HSQC-NMR (400 MHz, CDCl $)_{3}$ for compound $\mathbf{4 q}$ and $\mathbf{4 q ^ { \prime }}$

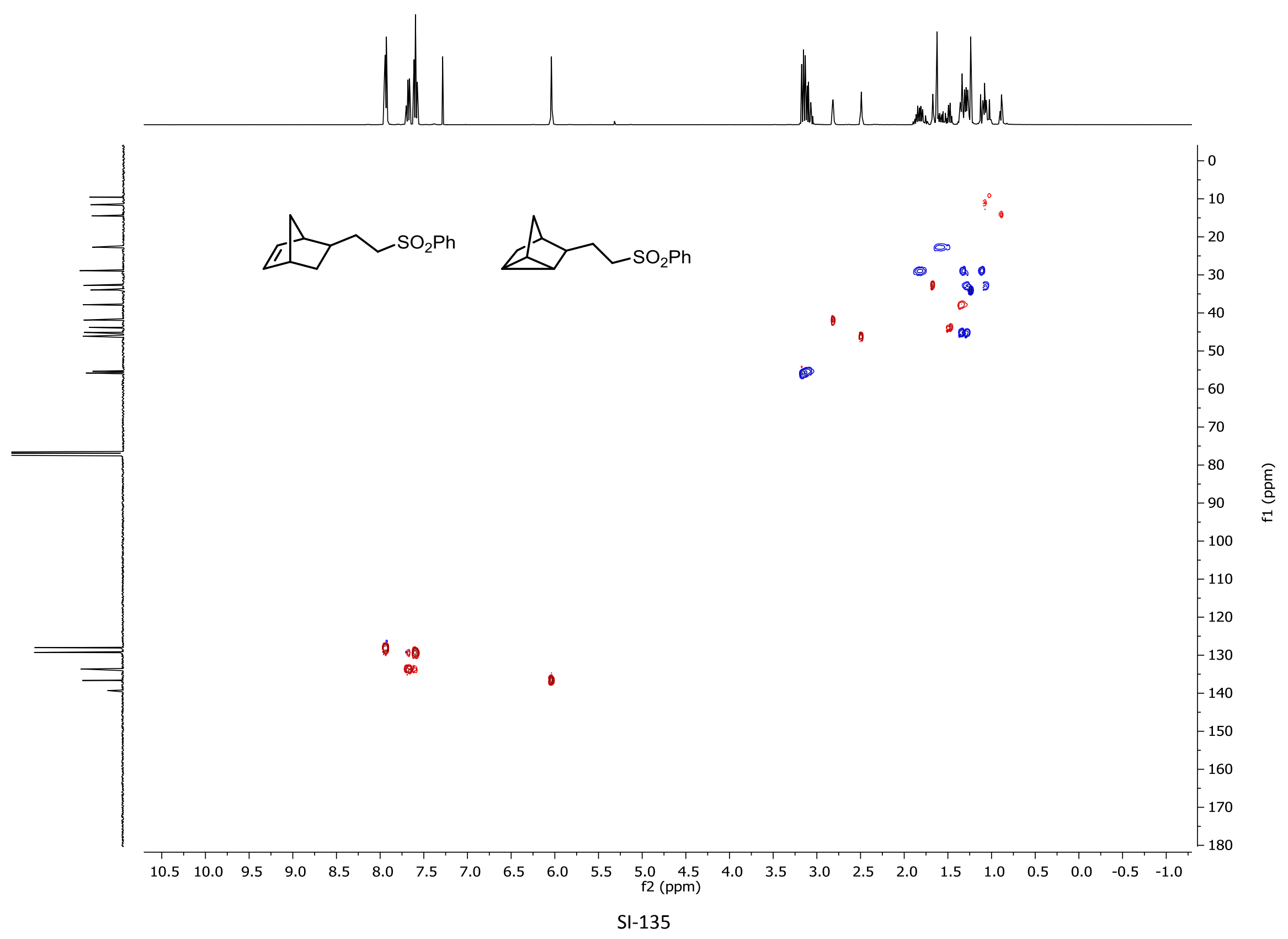




\section{${ }^{1} \mathrm{H}-\mathrm{NMR}\left(400 \mathrm{MHz}, \mathrm{CDCl}_{3}\right)$ for compound $4 r$}

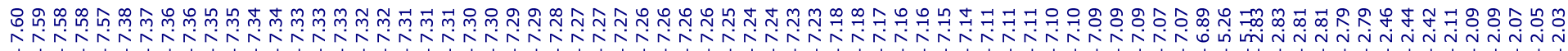

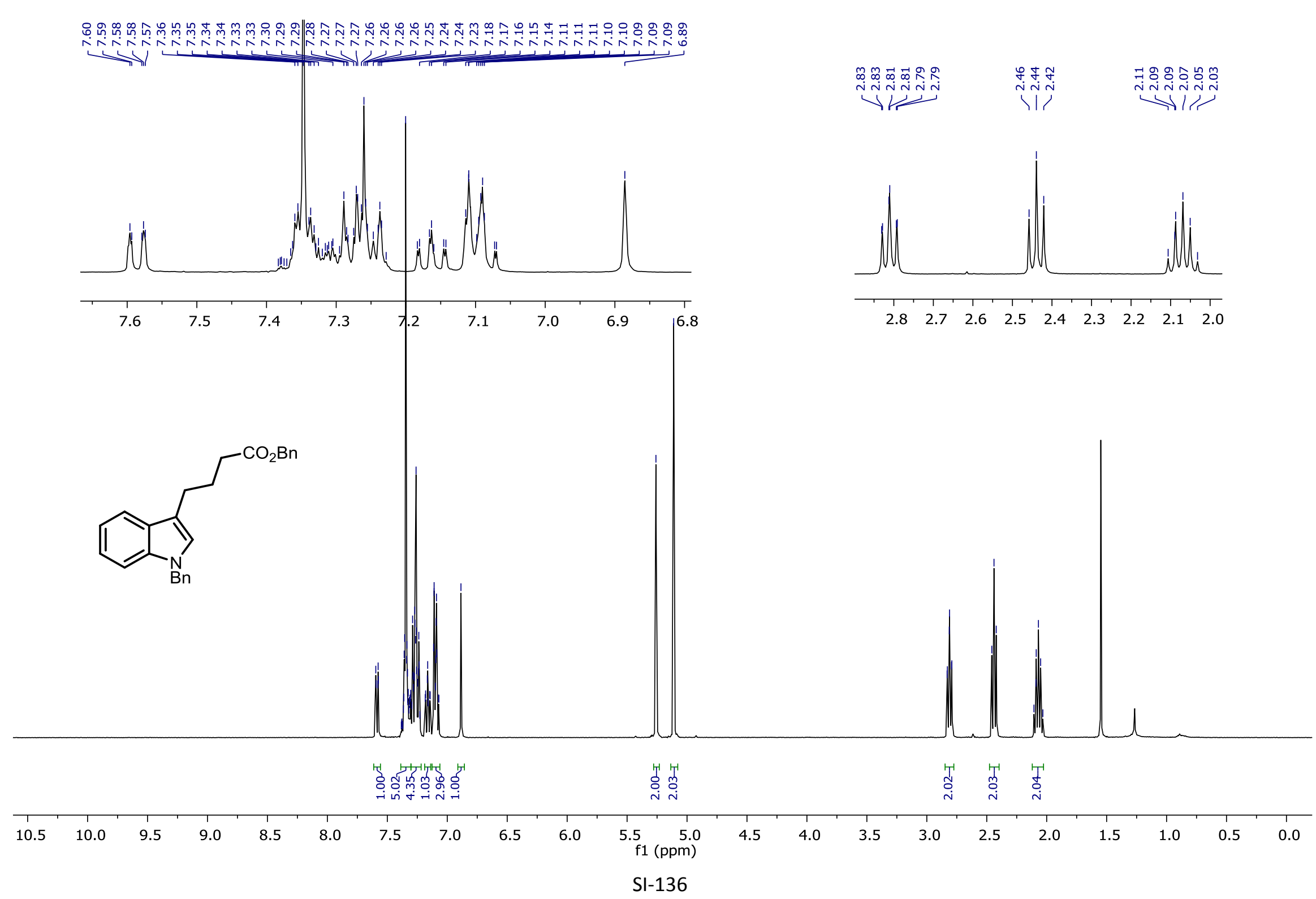


${ }^{13} \mathrm{C}-\mathrm{NMR}\left(101 \mathrm{MHz}, \mathrm{CDCl}_{3}\right)$ for compound $4 \mathrm{r}$
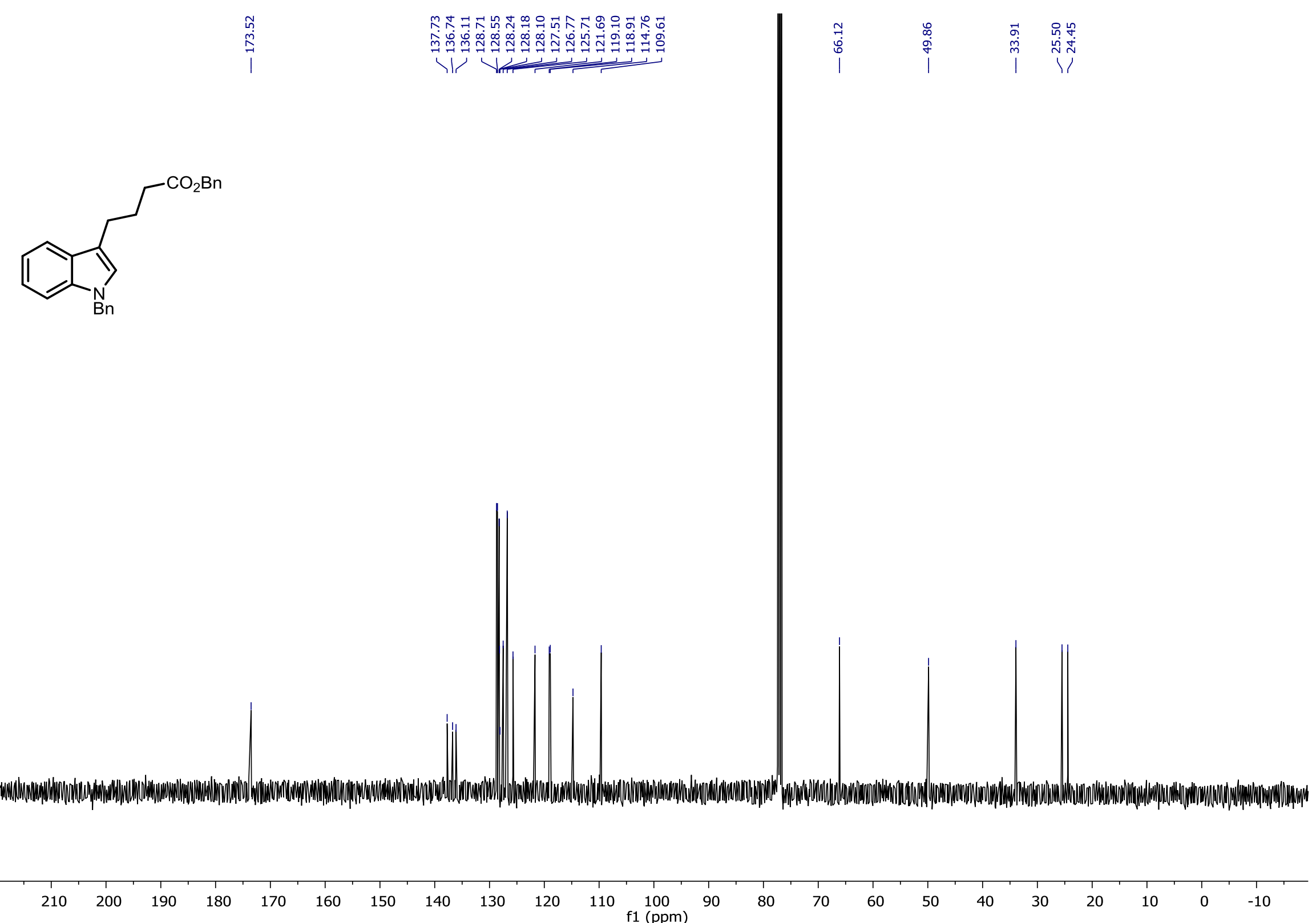

SI-137 


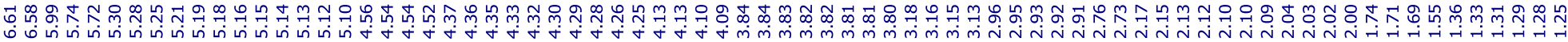
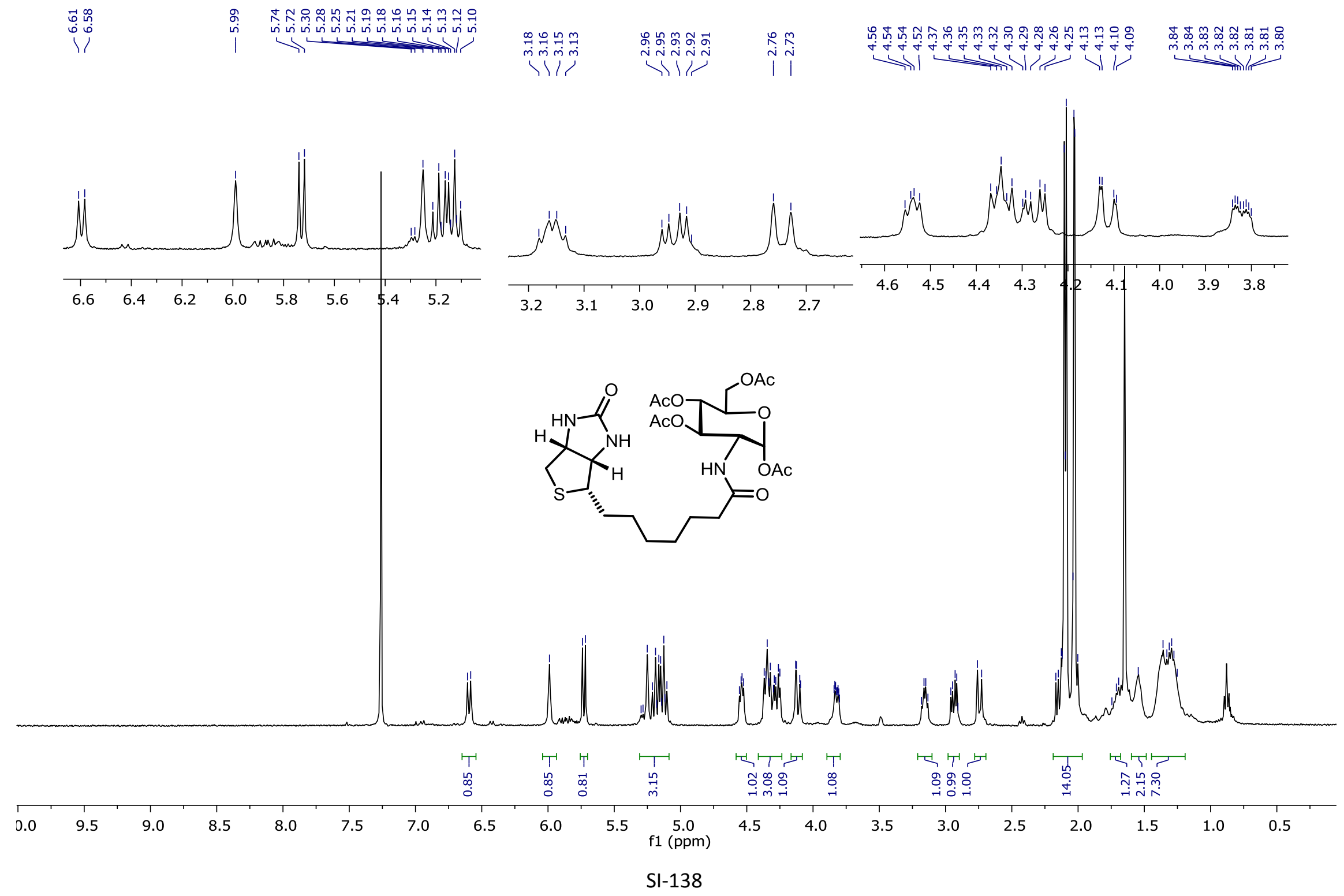
${ }^{13} \mathrm{C}-\mathrm{NMR}(101 \mathrm{MHz}, \mathrm{CDCl} 3)$ for compound $4 \mathrm{~s}$

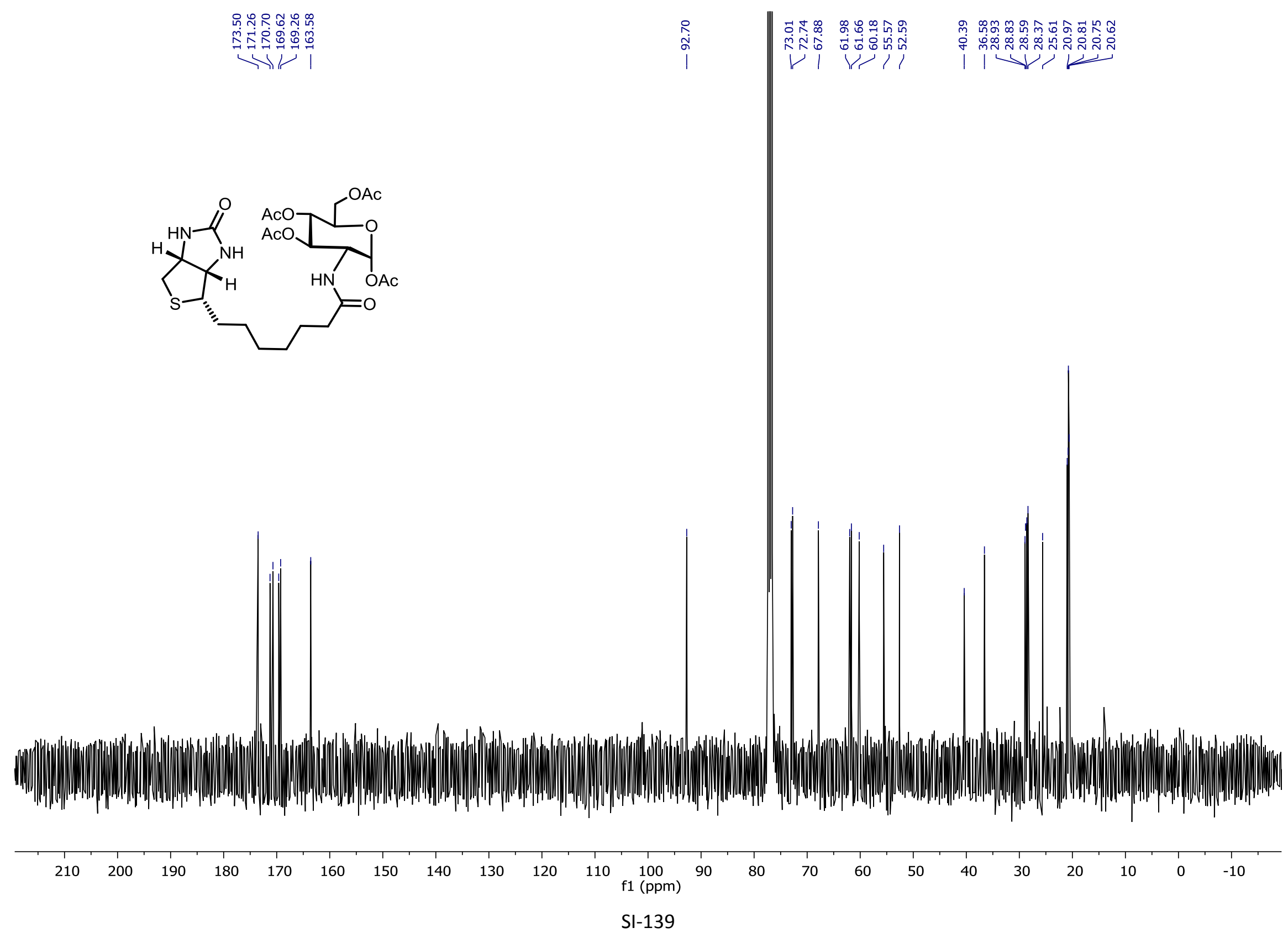




\section{${ }^{1} \mathrm{H}-\mathrm{NMR}\left(400 \mathrm{MHz}, \mathrm{CDCl}_{3}\right)$ for compound $4 \mathrm{t}$}

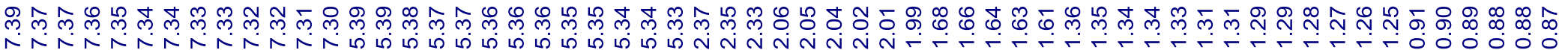
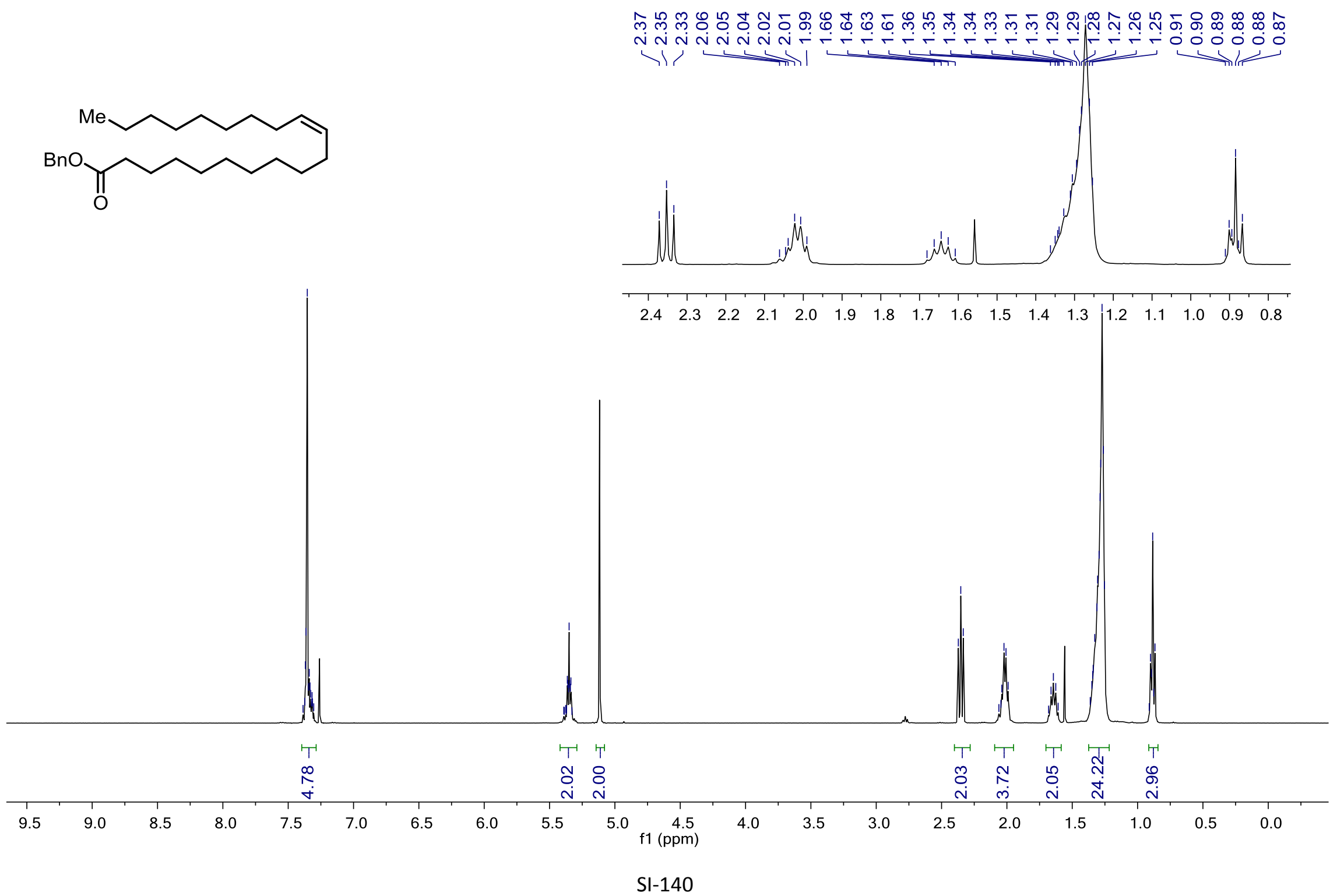
${ }^{13} \mathrm{C}-\mathrm{NMR}(101 \mathrm{MHz}, \mathrm{CDCl} 3)$ for compound $4 \boldsymbol{t}$

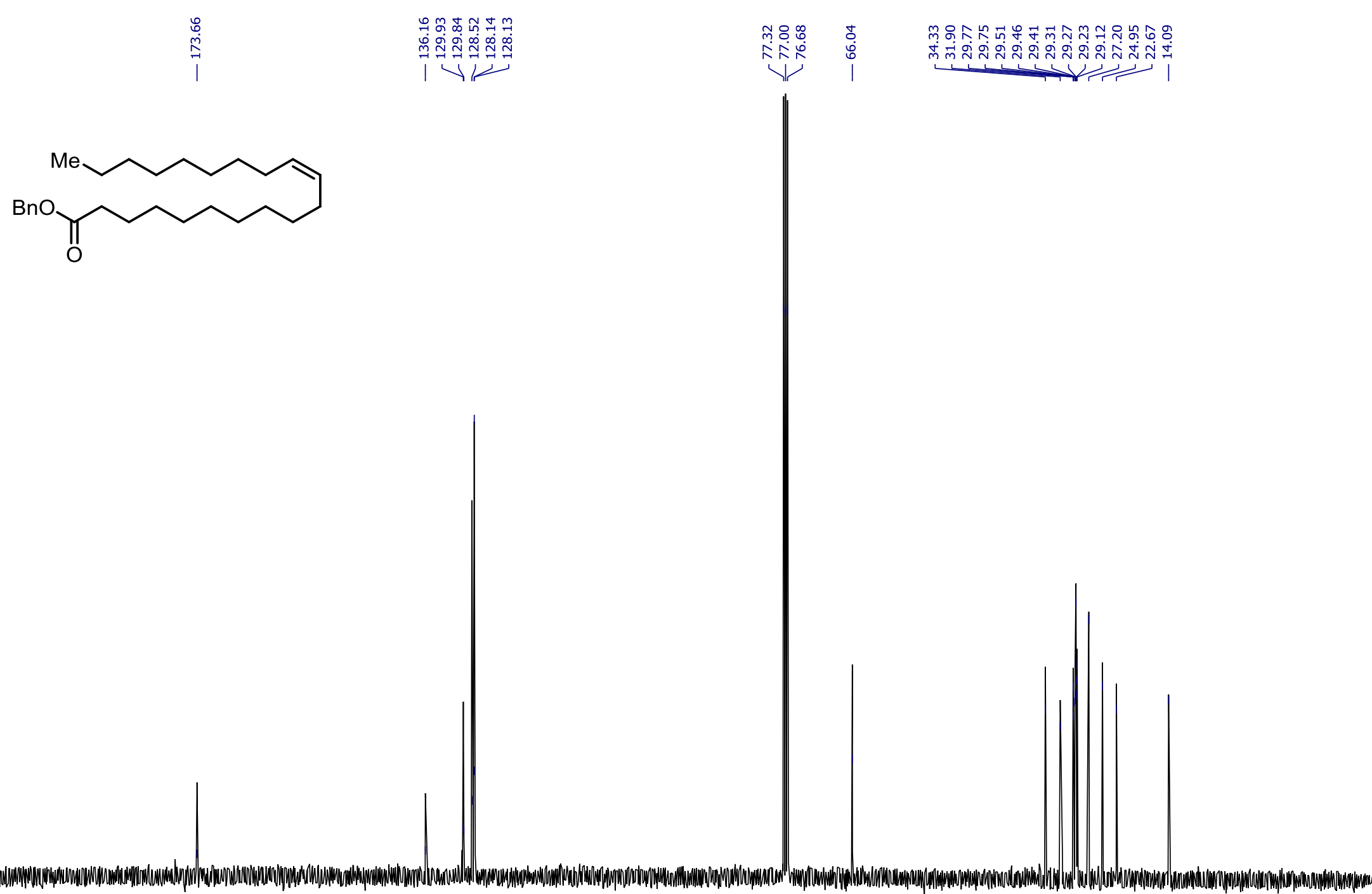

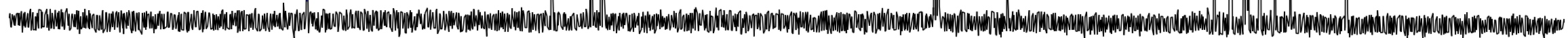

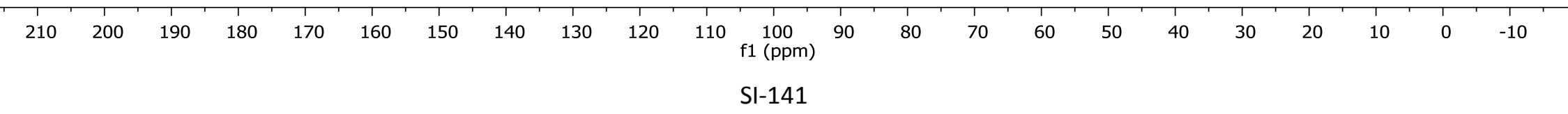




\section{${ }^{1} \mathrm{H}-\mathrm{NMR}\left(400 \mathrm{MHz}, \mathrm{CDCl}_{3}\right)$ for compound $4 \mathrm{u}$}

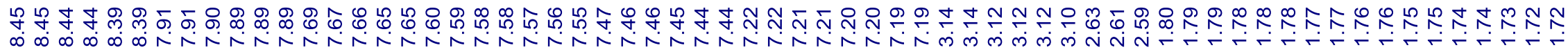

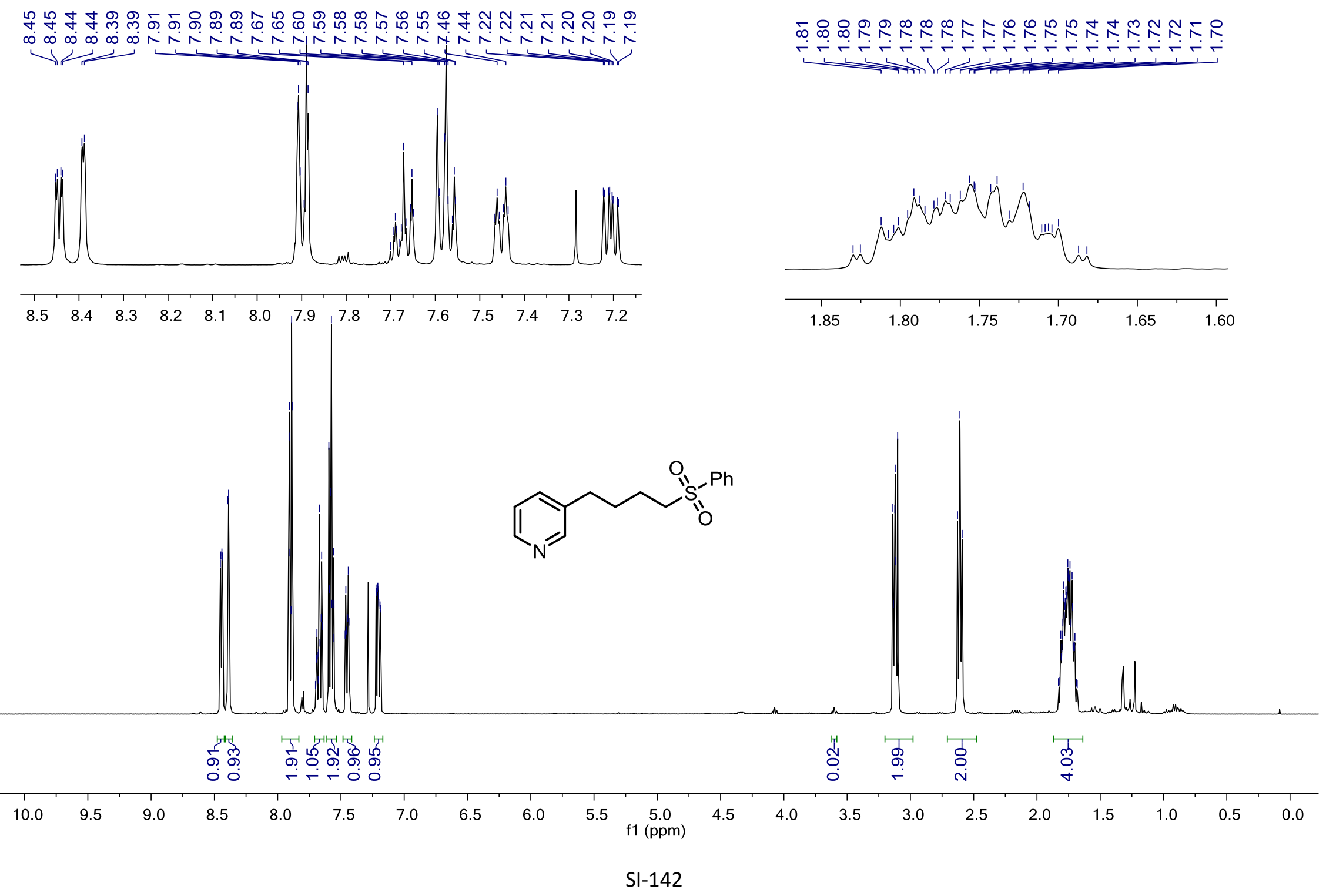


${ }^{13} \mathrm{C}-\mathrm{NMR}(101 \mathrm{MHz}, \mathrm{CDCl} / 3)$ for compound $\mathbf{4 u}$

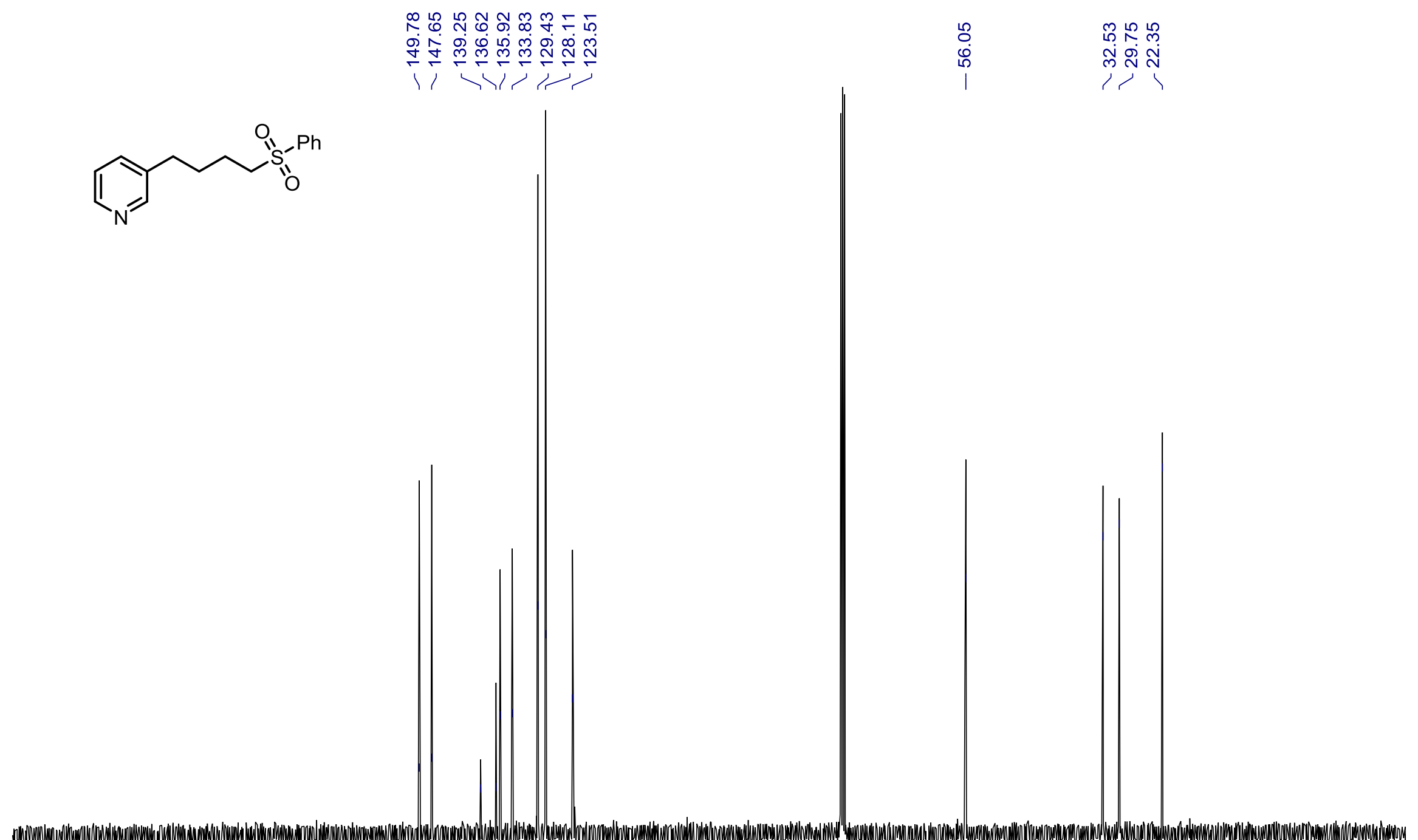

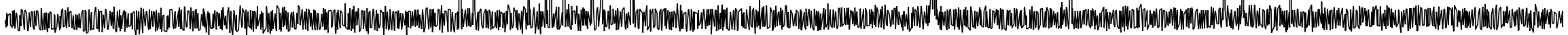

\begin{tabular}{|c|c|c|c|c|c|c|c|c|c|c|c|c|c|c|c|c|c|c|c|c|c|c|}
\hline \multirow[t]{2}{*}{210} & \multirow[t]{2}{*}{200} & \multirow[t]{2}{*}{190} & \multirow[t]{2}{*}{180} & \multirow[t]{2}{*}{170} & \multirow[t]{2}{*}{160} & \multirow[t]{2}{*}{150} & \multirow[t]{2}{*}{140} & \multirow[t]{2}{*}{130} & \multirow[t]{2}{*}{120} & \multicolumn{2}{|c|}{$\begin{array}{cc}110 & 100 \\
\mathrm{f} 1(\mathrm{ppm})\end{array}$} & & 80 & 70 & 60 & 50 & 40 & 30 & 20 & 10 & \multirow[t]{2}{*}{0} & \multirow[t]{2}{*}{-10} \\
\hline & & & & & & & & & & & SI-143 & & & & & & & & & & & \\
\hline
\end{tabular}



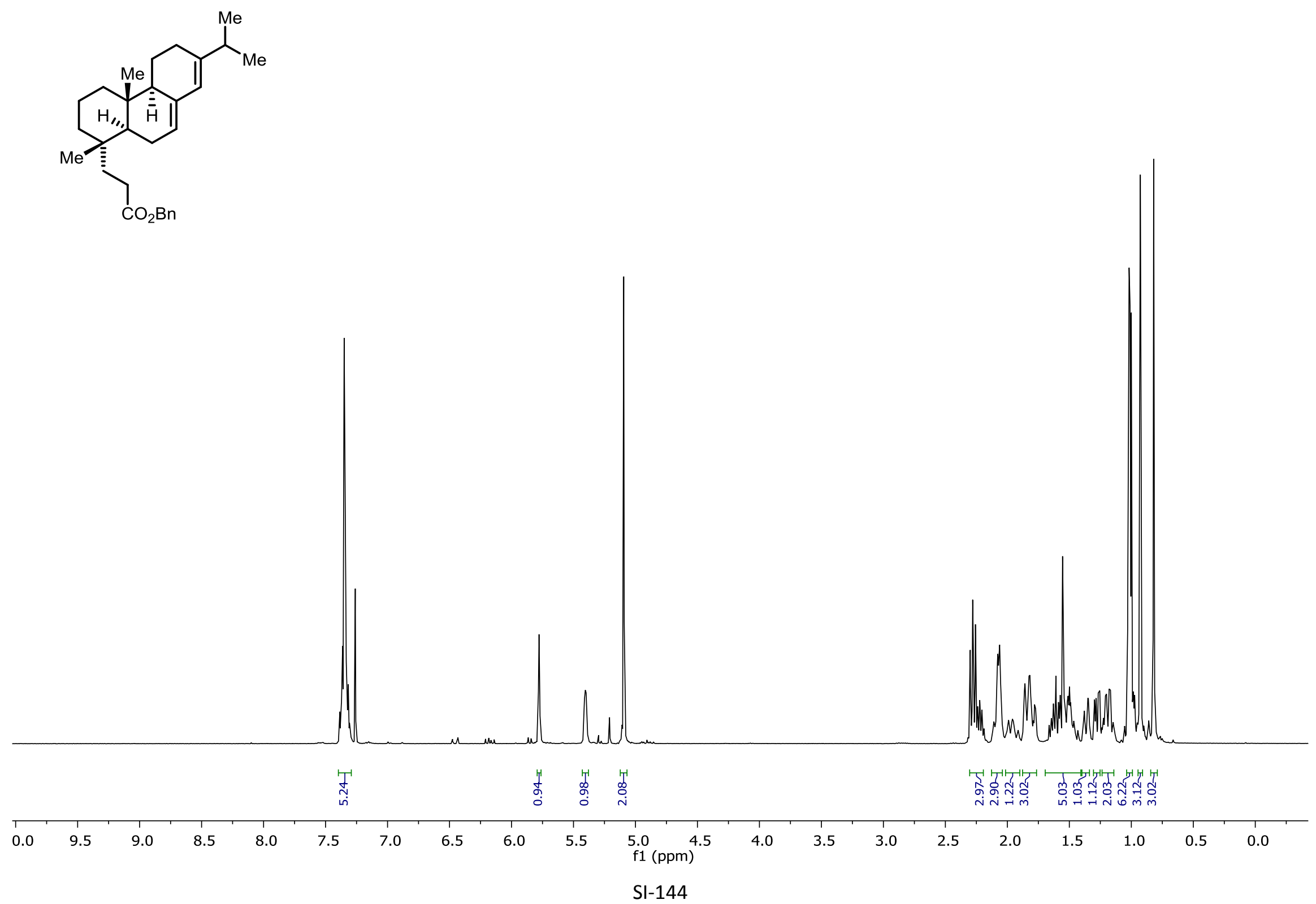
${ }^{13} \mathrm{C}-\mathrm{NMR}\left(101 \mathrm{MHz}, \mathrm{CDCl}_{3}\right)$ for compound $\mathbf{4 v}$

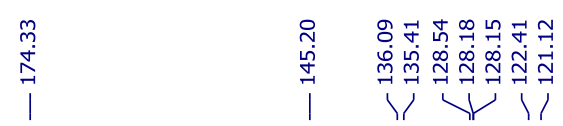

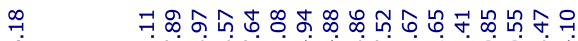

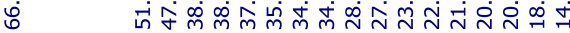

$1<1<11$
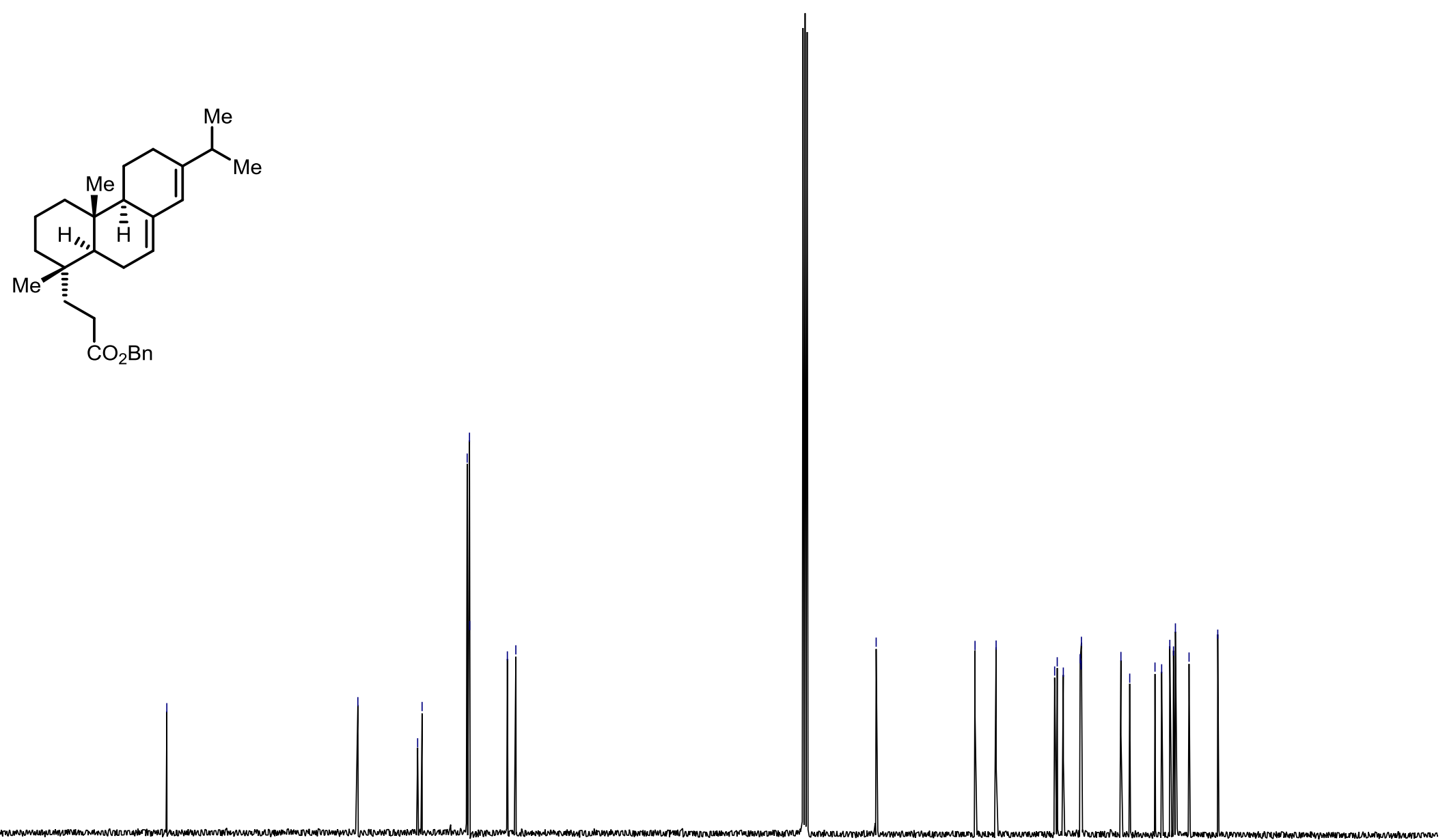

$210 \quad 200$ 


\section{${ }^{1} \mathrm{H}-\mathrm{NMR}\left(400 \mathrm{MHz}, \mathrm{CDCl}_{3}\right)$ for compound $4 \mathrm{w}$}

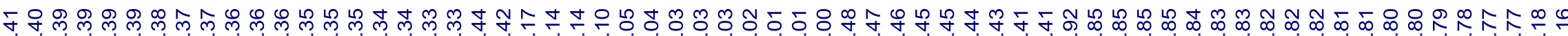

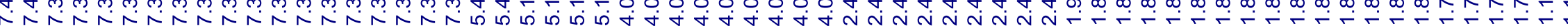

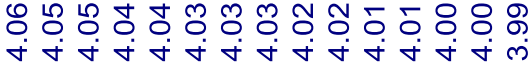

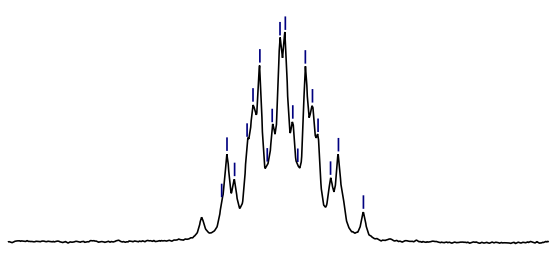

$\begin{array}{lllllll}4.20 & 4.15 & 4.10 & 4.05 & 4.00 & 3.95 & 3.90\end{array}$

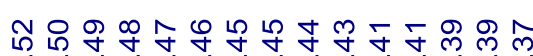
hinininininisin

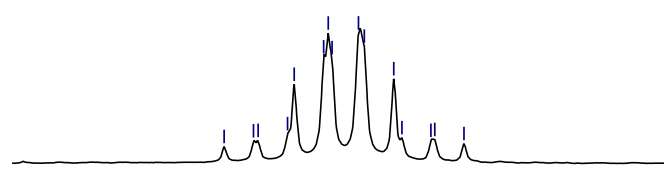

$\begin{array}{lllllll}2.60 & 2.55 & 2.50 & 2.45 & 2.40 & 2.35 & 2.30\end{array}$<smiles>CC(C)CCC(=O)OCc1ccccc1</smiles>

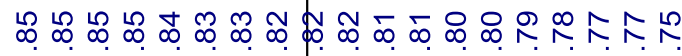
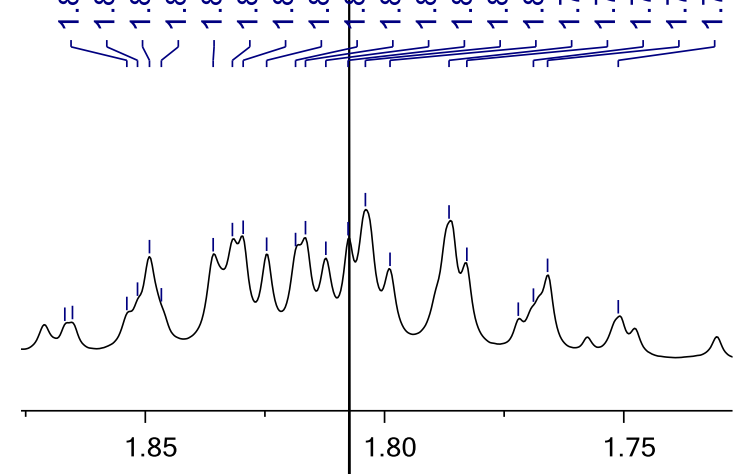

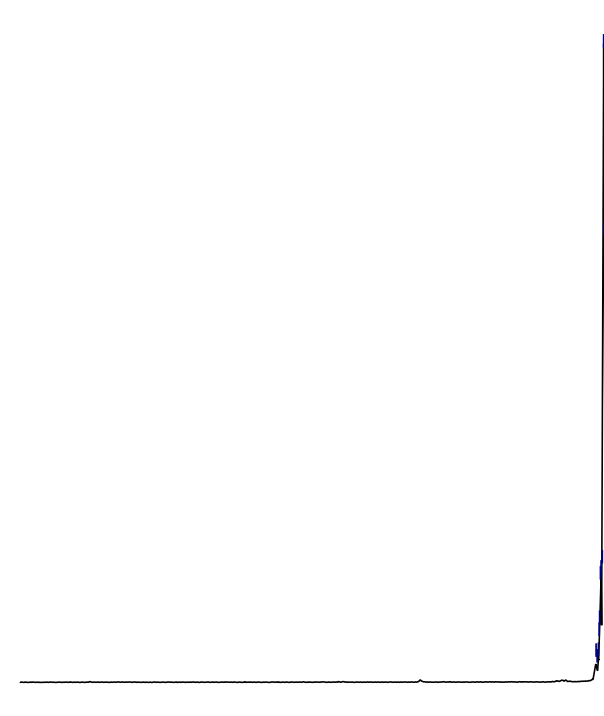

(1)

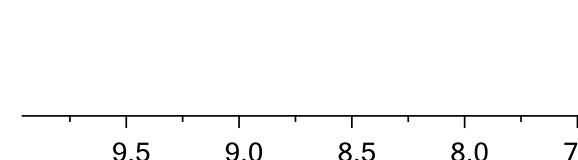

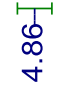

กำ

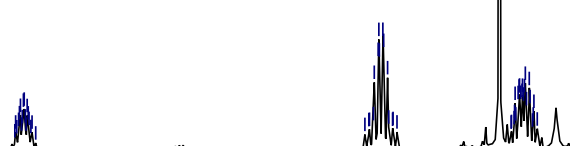

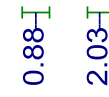

(1)

'ำ

9.5
9.0

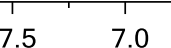

6.5

6.0

5.0
$\mathrm{f} 1(\mathrm{p}$

5.0
f1 (ppm)

$4.5 \quad 4.0$

SI-146 
${ }^{13} \mathrm{C}-\mathrm{NMR}(101 \mathrm{MHz}, \mathrm{CDCl})$ for compound $\mathbf{4 w}$

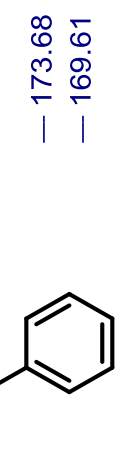

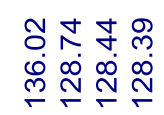<smiles>CC(CCC(=O)OCc1ccccc1)NC(C)(C)C</smiles>

$\mid$ |
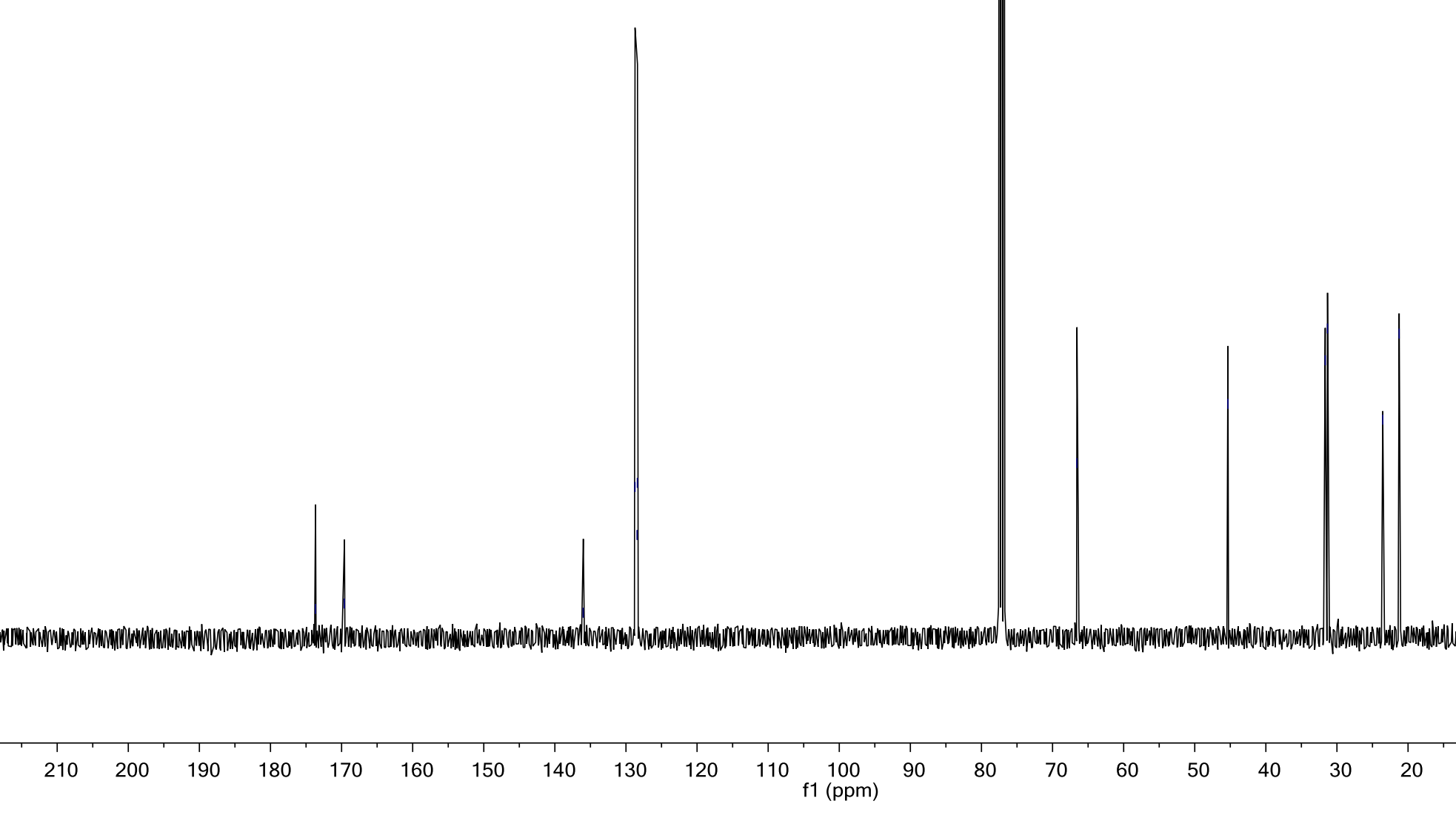


\section{${ }^{1} \mathrm{H}-\mathrm{NMR}\left(400 \mathrm{MHz}, \mathrm{CDCl}_{3}\right)$ for compound $\mathbf{4 x}$}

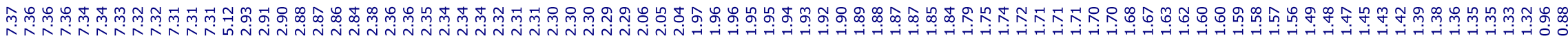

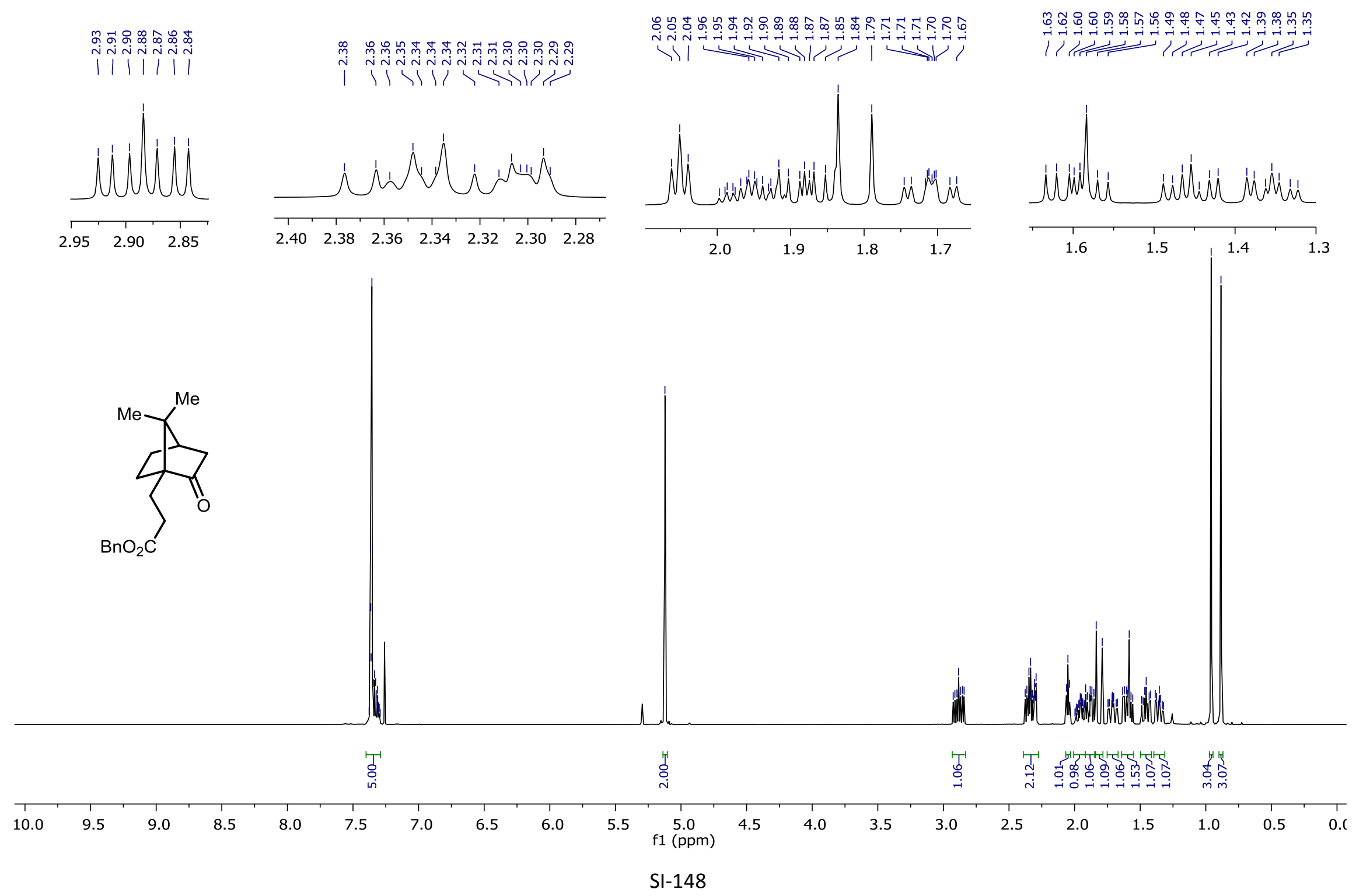


${ }^{13} \mathrm{C}-\mathrm{NMR}(101 \mathrm{MHz}, \mathrm{CDCl} / 3)$ for compound $4 \mathrm{x}$

$\stackrel{3}{0}$
$\dot{0}$
$\stackrel{1}{v}$
$\mid$

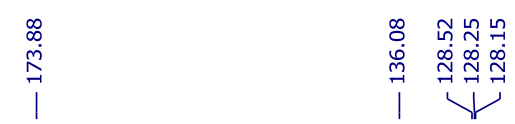

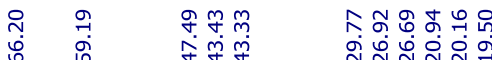

i
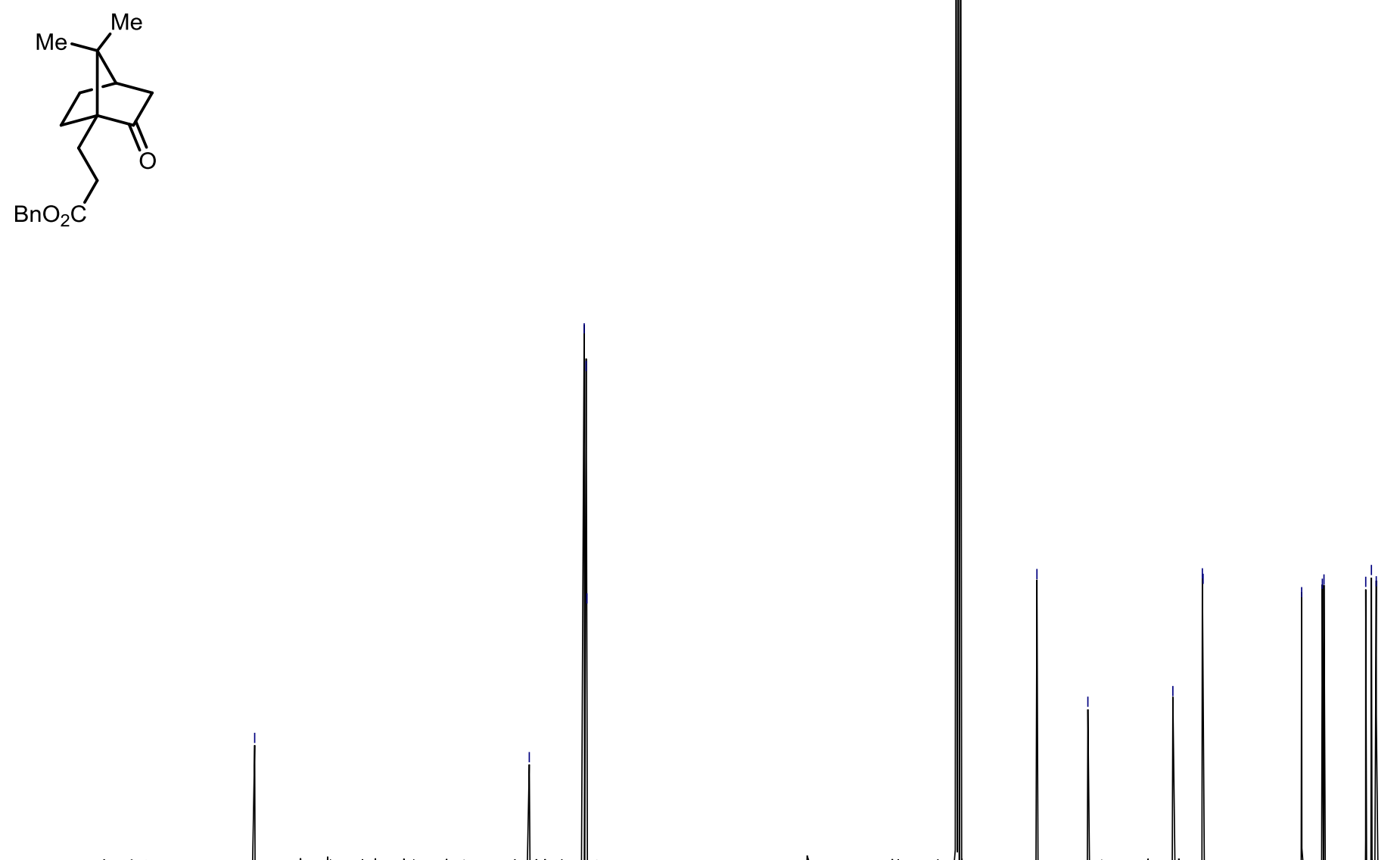

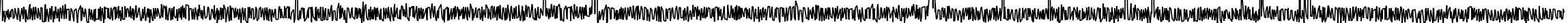

\begin{tabular}{|c|c|c|c|c|c|c|c|c|c|c|c|c|c|c|c|c|c|c|c|c|c|c|}
\hline 210 & 200 & 190 & 180 & 170 & 160 & 150 & 140 & 130 & 120 & 110 & $\begin{array}{c}100 \\
\mathrm{f} 1(\mathrm{ppm})\end{array}$ & 90 & 80 & 70 & 60 & 50 & 40 & 30 & 20 & 10 & 0 & -10 \\
\hline & & & & & & & & & & & SI-149 & & & & & & & & & & & \\
\hline
\end{tabular}


${ }^{1} \mathrm{H}-\mathrm{NMR}\left(400 \mathrm{MHz}, \mathrm{CDCl}_{3}\right)$ for compound $4 \mathrm{y}$

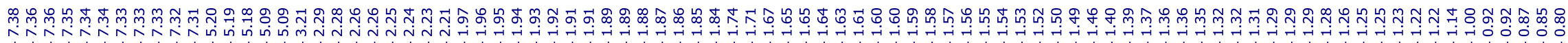

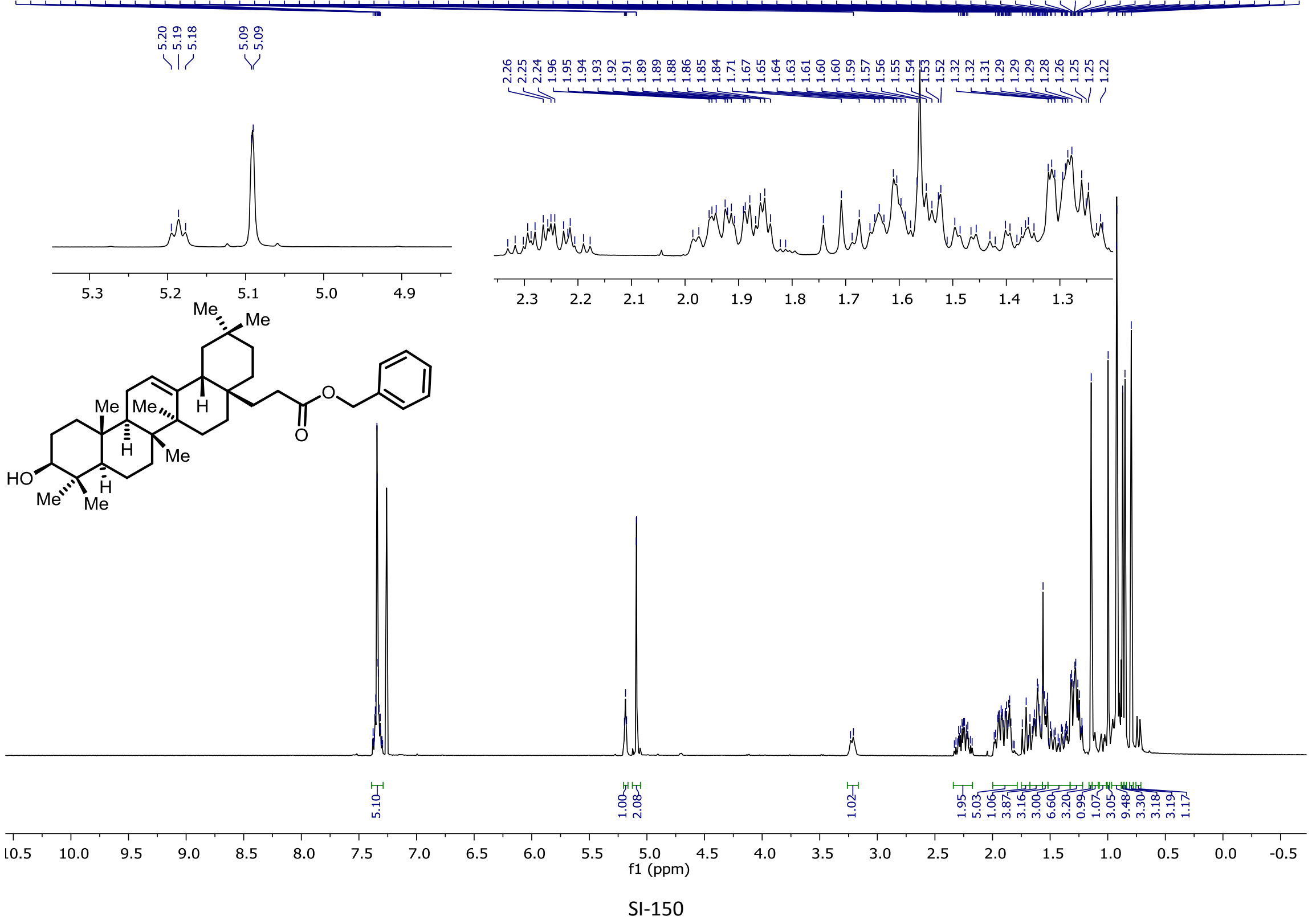


${ }^{13} \mathrm{C}-\mathrm{NMR}(101 \mathrm{MHz}, \mathrm{CDCl} / 3)$ for compound $4 \boldsymbol{y}$
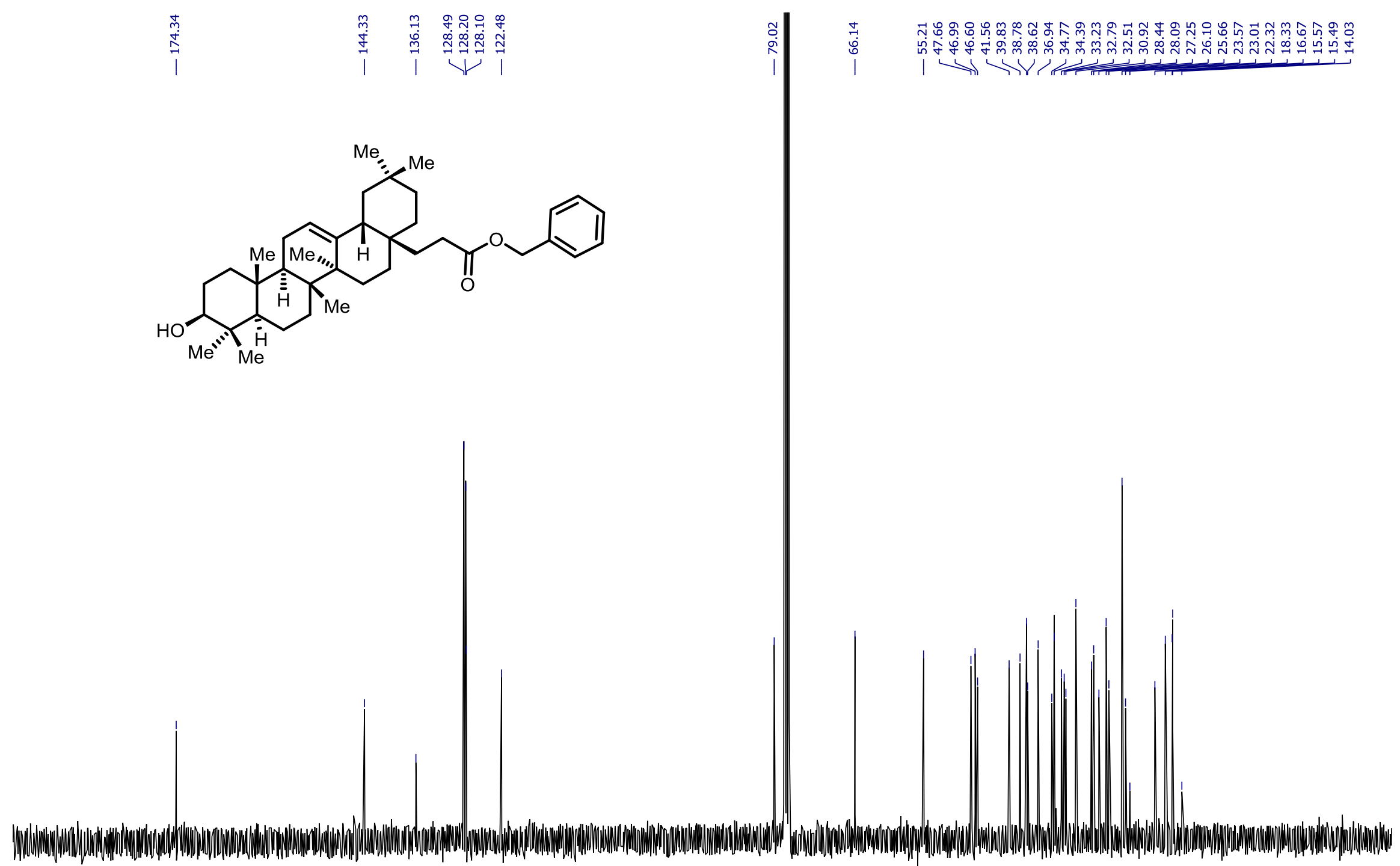


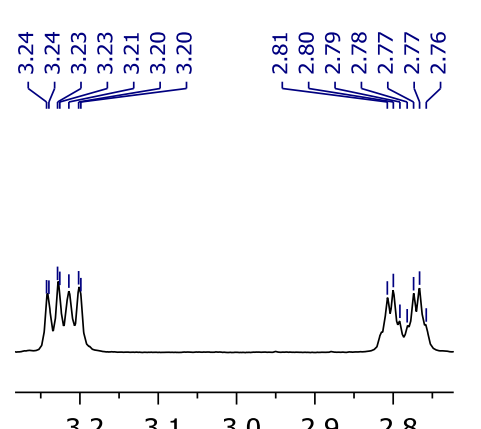

$\begin{array}{lllll}3.2 & 3.1 & 3.0 & 2.9 & 2.8\end{array}$

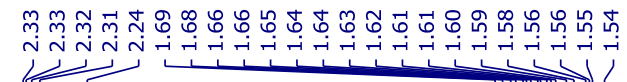

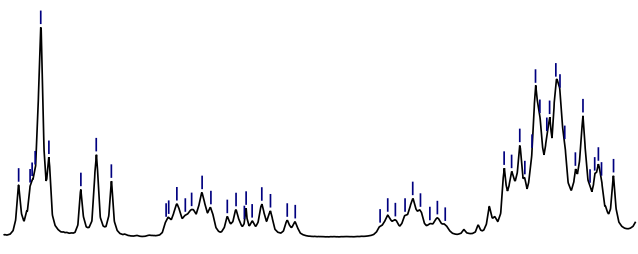

$\begin{array}{llllllll}2.3 & 2.2 & 2.1 & 2.0 & 1.9 & 1.8 & 1.7 & 1.6\end{array}$

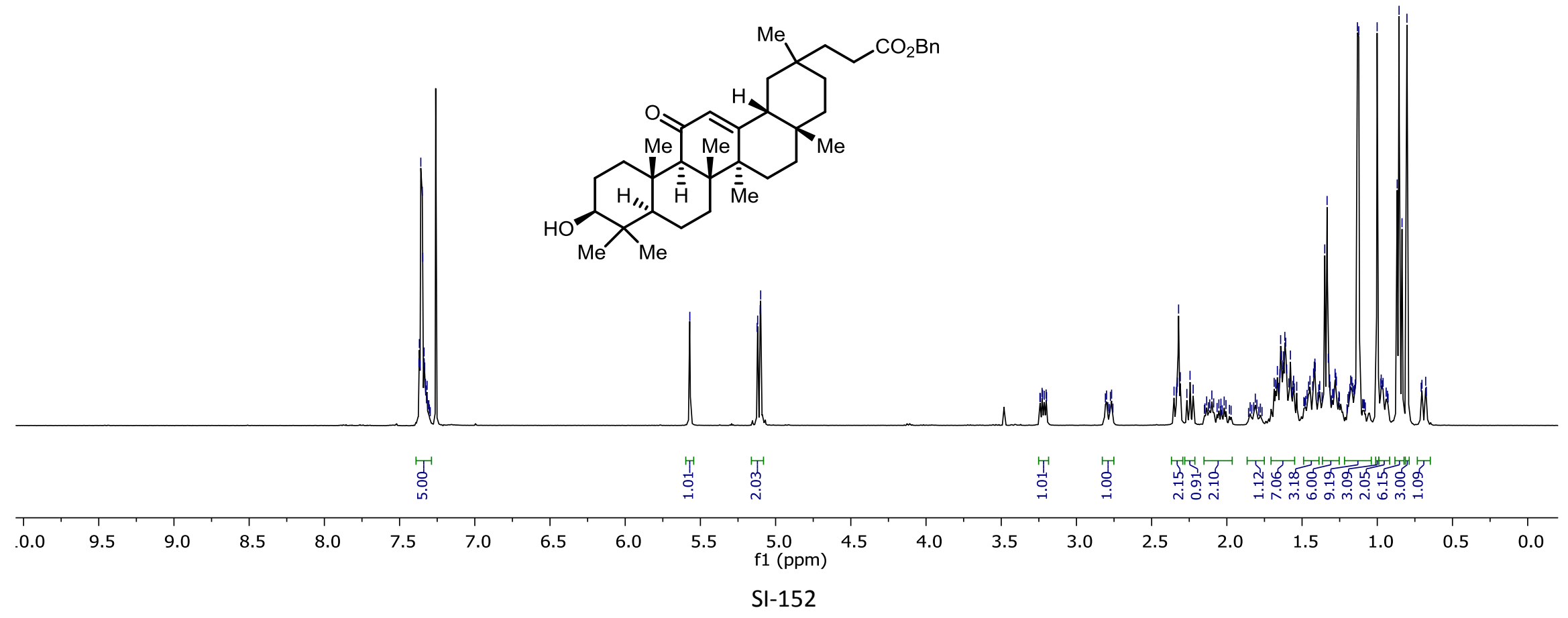

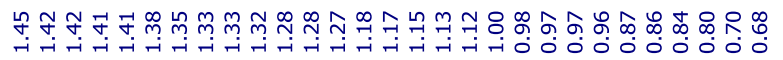
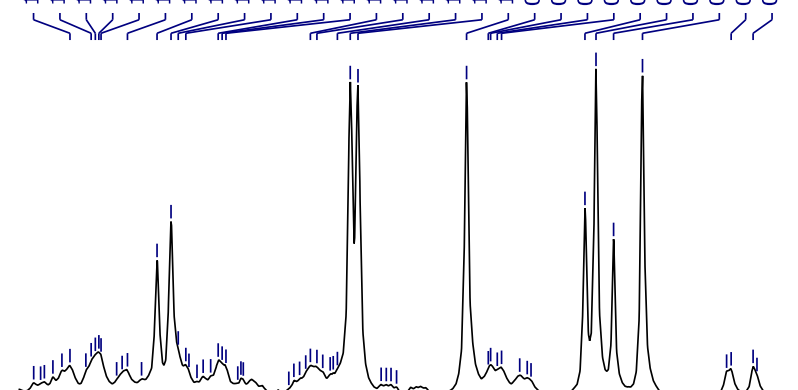

$\begin{array}{lllllllll}1.5 & 1.4 & 1.3 & 1.2 & 1.1 & 1.0 & 0.9 & 0.8 & 0.7\end{array}$ 
${ }^{13} \mathrm{C}-\mathrm{NMR}(101 \mathrm{MHz}, \mathrm{CDCl} 3)$ for compound $\mathbf{4 z}$

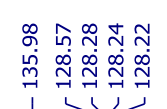

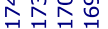

N

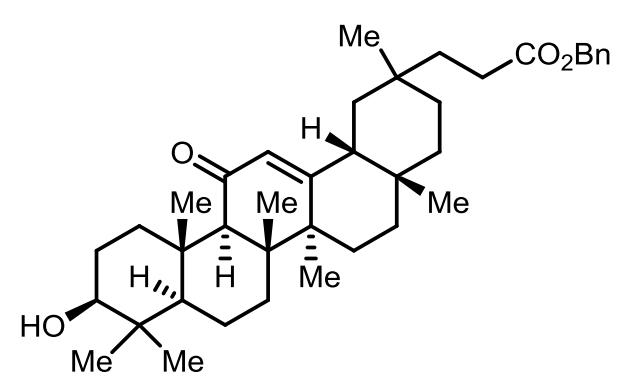

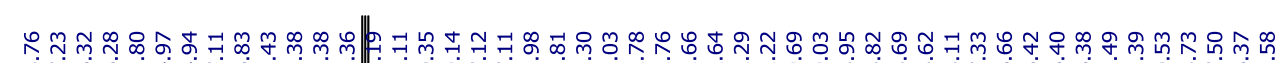

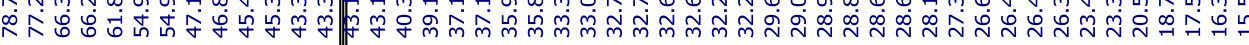

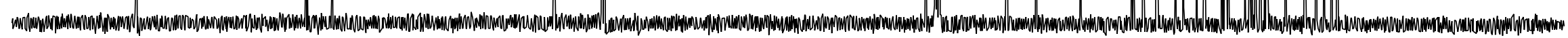

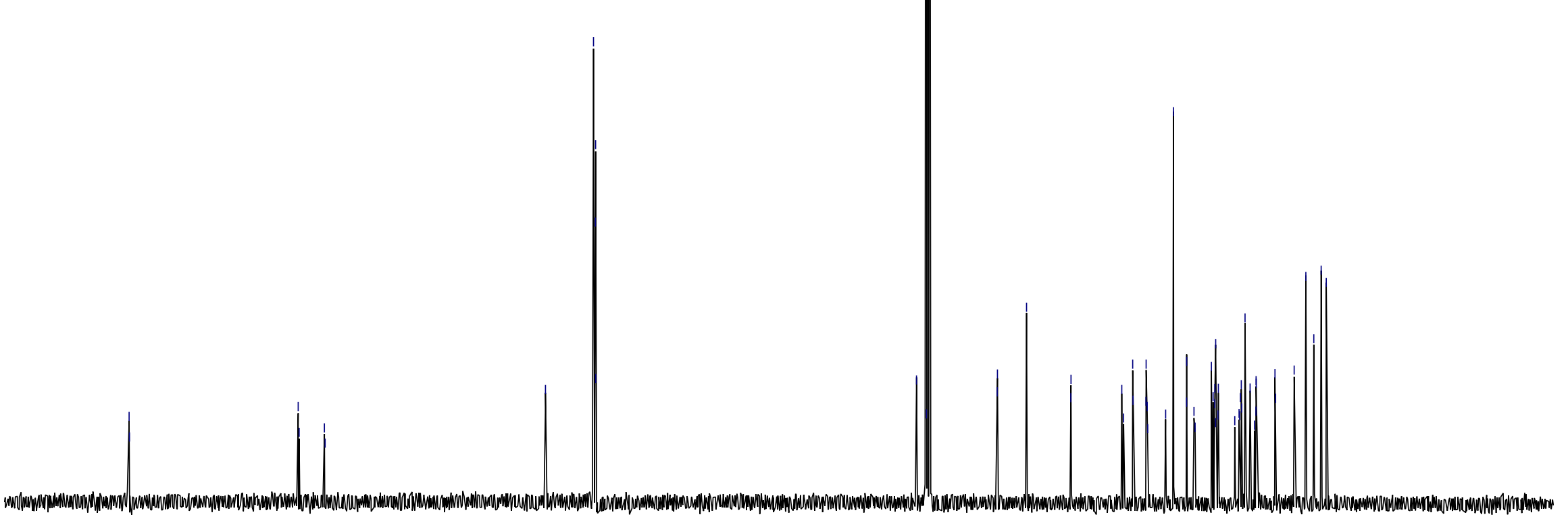

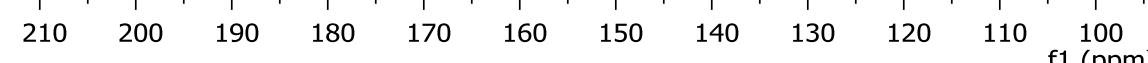

80

$70 \quad 60$

50

40

SI-153 


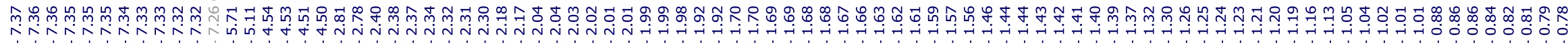

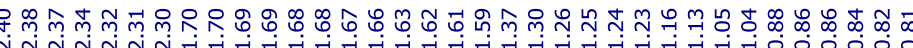

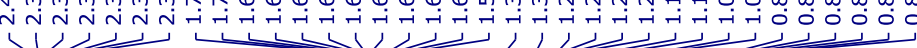

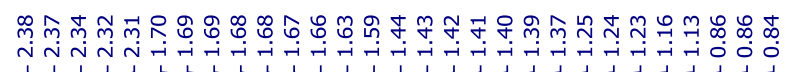

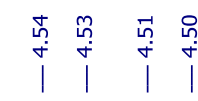
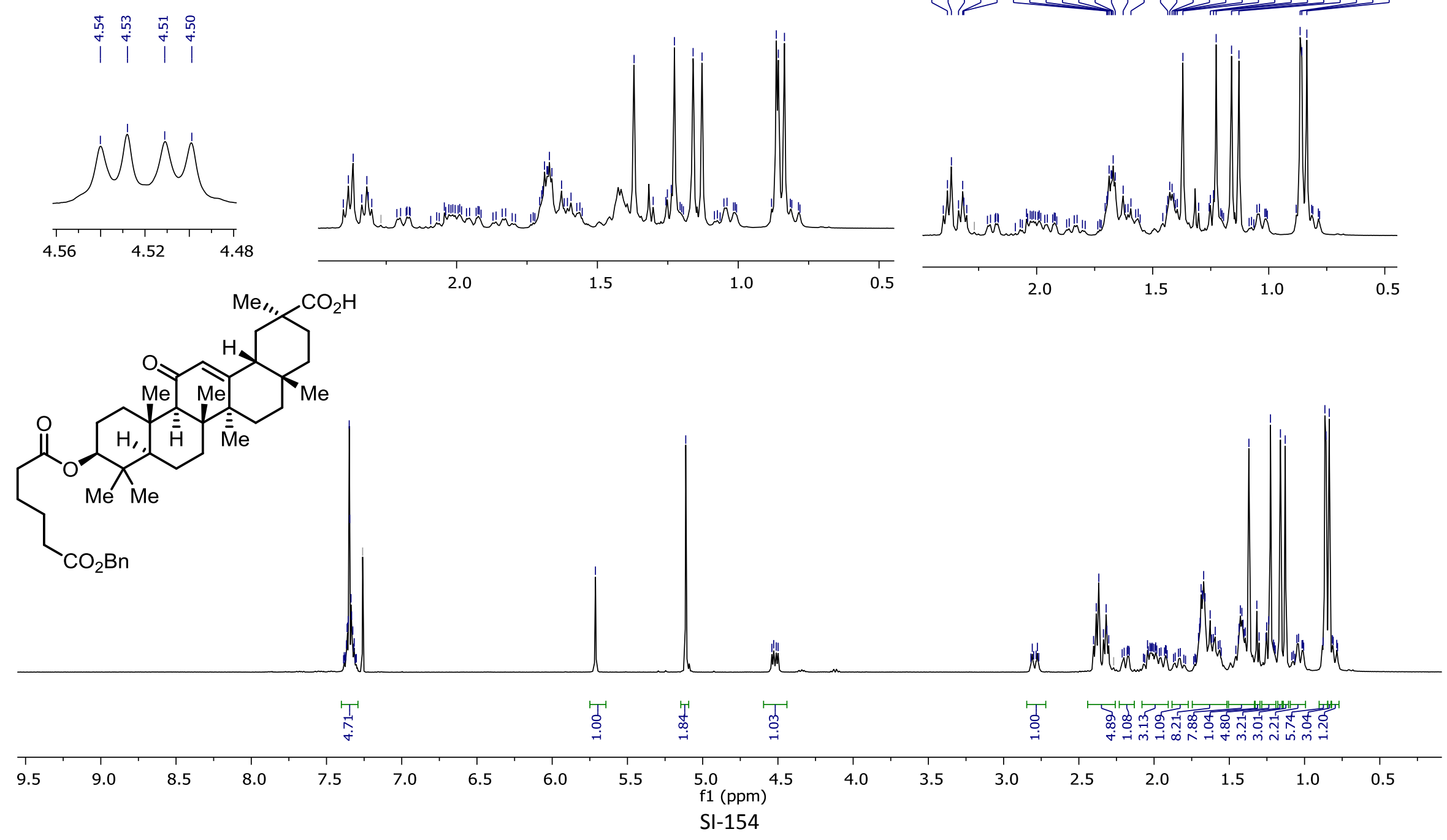
${ }^{13} \mathrm{C}-\mathrm{NMR}\left(400 \mathrm{MHz}, \mathrm{CDCl}_{3}\right)$ for compound $4 \mathrm{aa}$

|

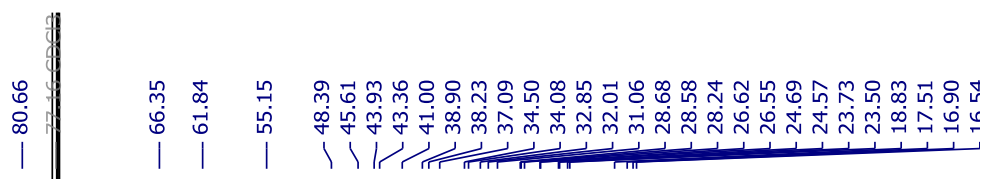
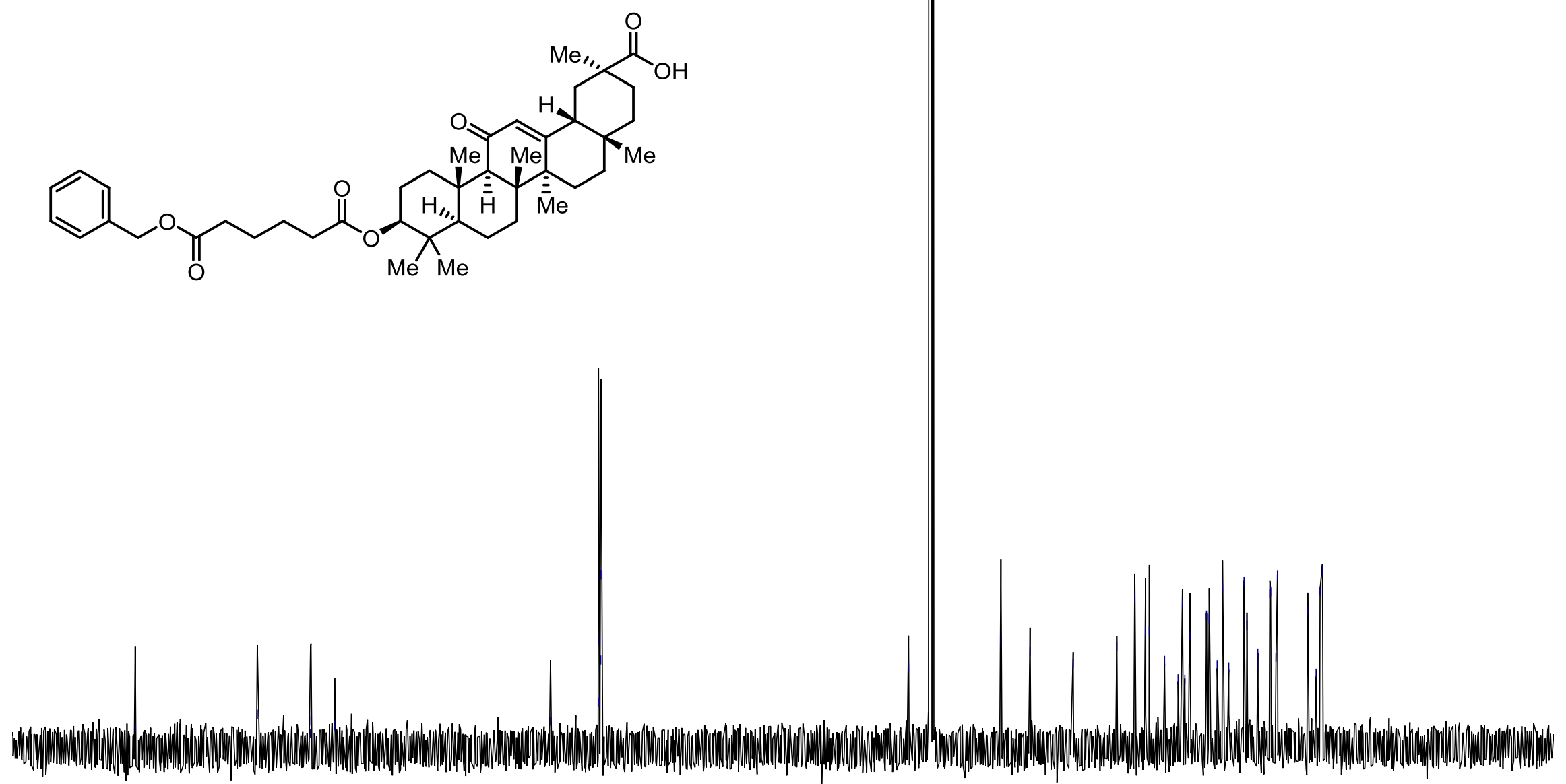

$210 \quad 200$

$170 \quad 160$

$150 \quad 140$

$120 \quad 110$

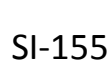

$80 \quad 70 \quad 60$

50

40 
DEPT $135 \mathrm{NMR}\left(101 \mathrm{MHz}, \mathrm{CDCl}_{3}\right)$ for compound $4 a a$

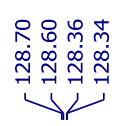

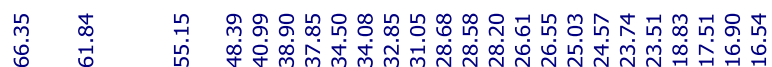

i i
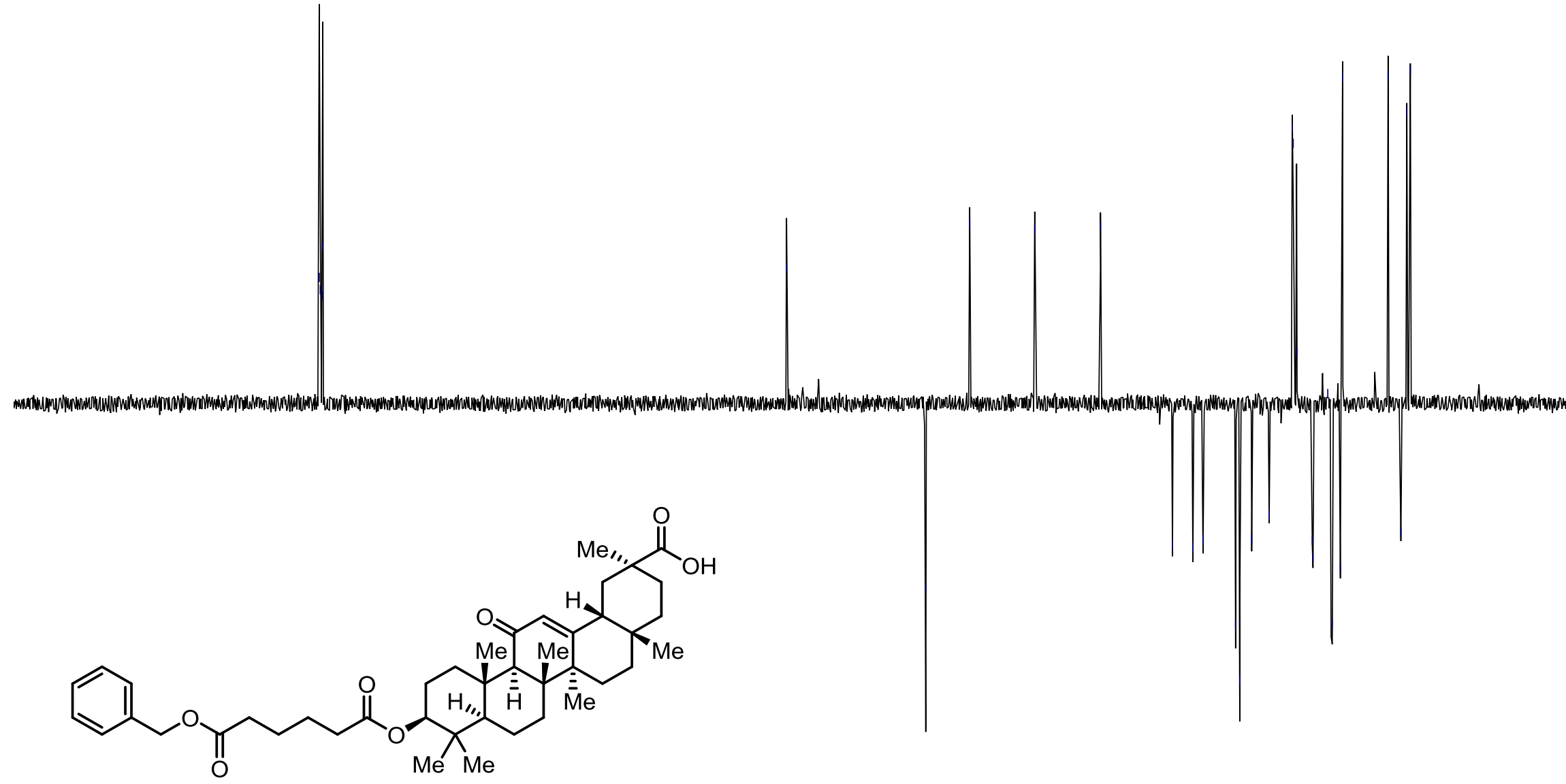

150 140 130 120 110 100 90 
HSQC NMR $\left(\mathrm{CDCl}_{3}\right)$ for compound 4aa

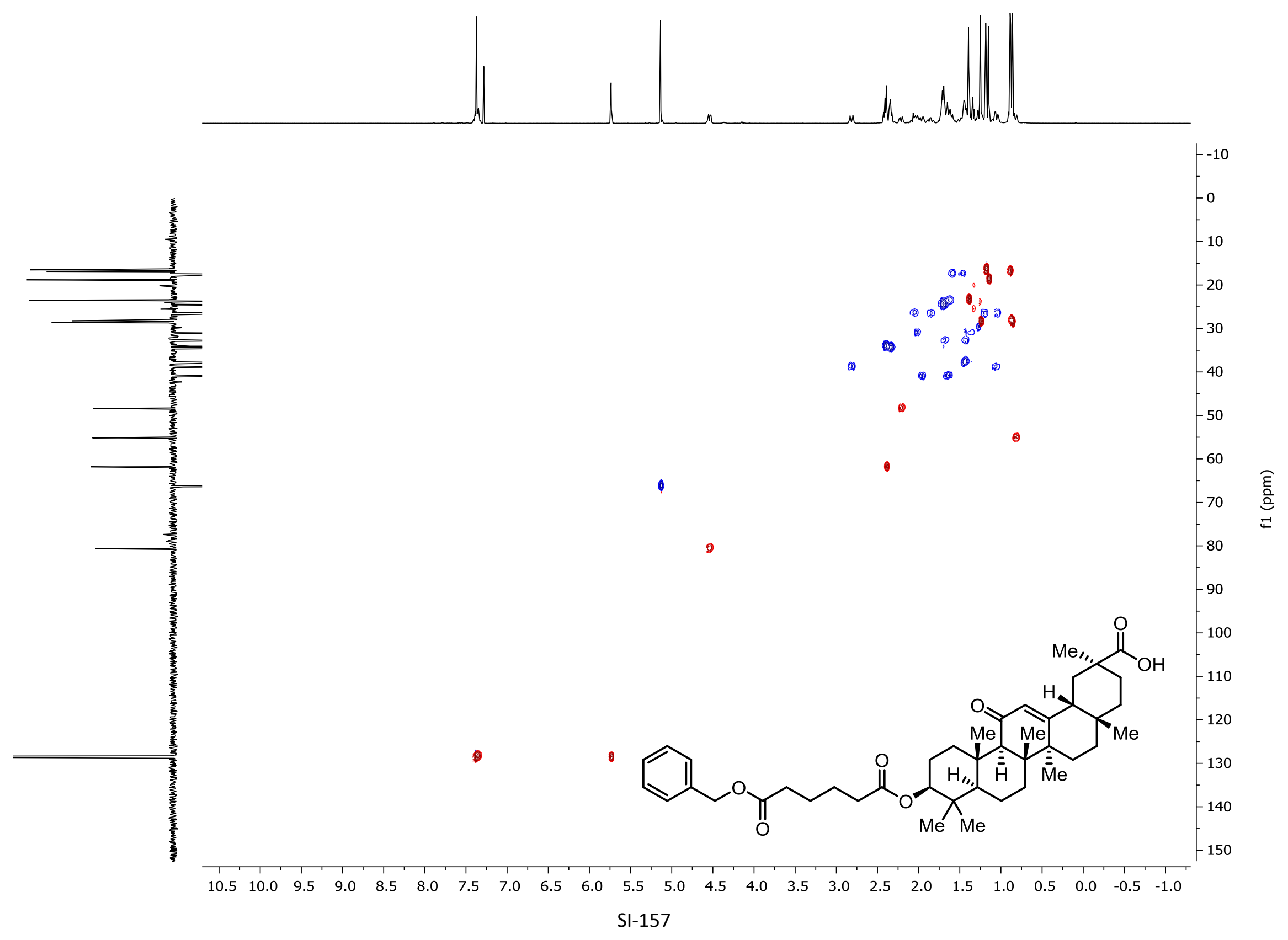




\section{${ }^{1} \mathrm{H}-\mathrm{NMR}\left(400 \mathrm{MHz}, \mathrm{CDCl}_{3}\right)$ for compound $4 a b$}

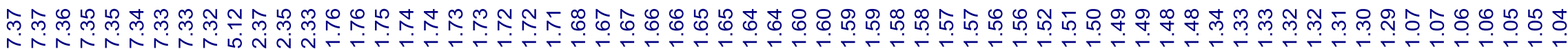

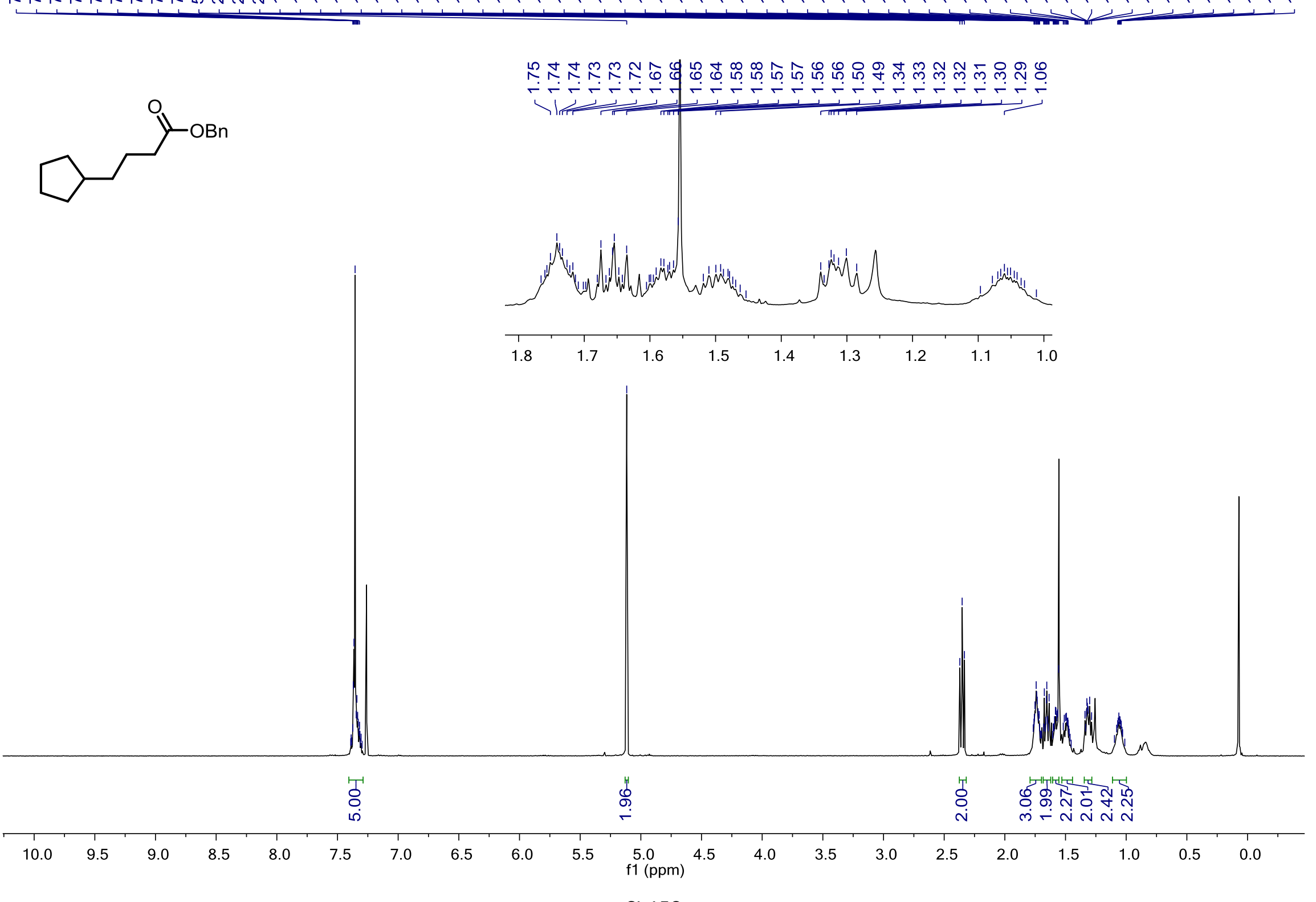

SI-158 
${ }^{13} \mathrm{C}-\mathrm{NMR}\left(101 \mathrm{MHz}, \mathrm{CDCl}_{3}\right.$ ) for compound $\mathbf{4 a b}$

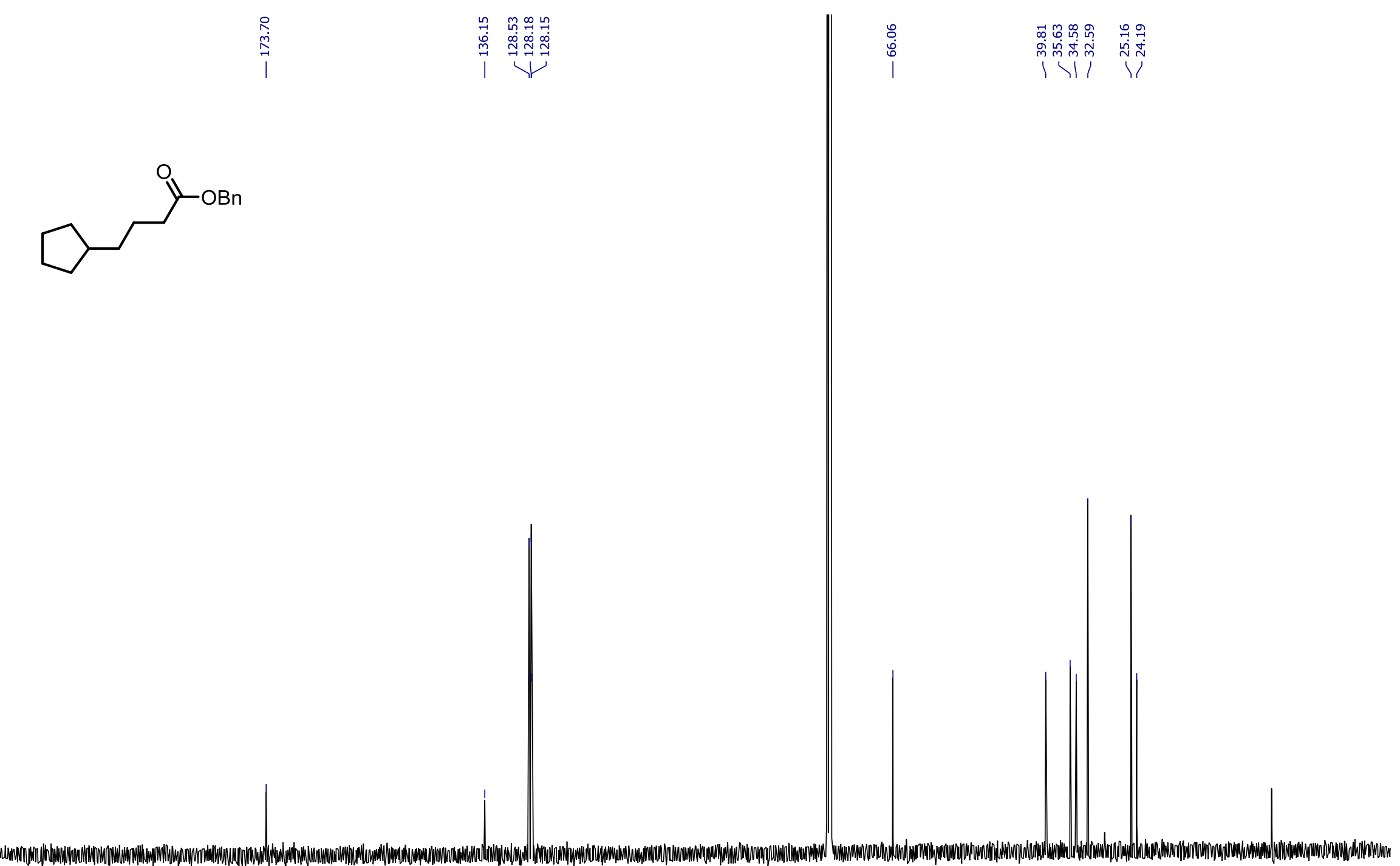

\begin{tabular}{|c|c|c|c|c|c|c|c|c|c|c|c|c|c|c|c|c|c|c|c|c|c|c|}
\hline 210 & 200 & 190 & 180 & 170 & 160 & 150 & 140 & 130 & 120 & 110 & $\begin{array}{c}100 \\
\mathrm{f} 1(\mathrm{ppm})\end{array}$ & 90 & 80 & 70 & 60 & 50 & 40 & 30 & 20 & 10 & 0 & -10 \\
\hline & & & & & & & & & & & SI-159 & & & & & & & & & & & \\
\hline
\end{tabular}


${ }^{1} \mathrm{H}-\mathrm{NMR}\left(400 \mathrm{MHz}, \mathrm{CDCl}_{3}\right.$ ) for compound $\mathbf{4 a \boldsymbol { b }}$ '

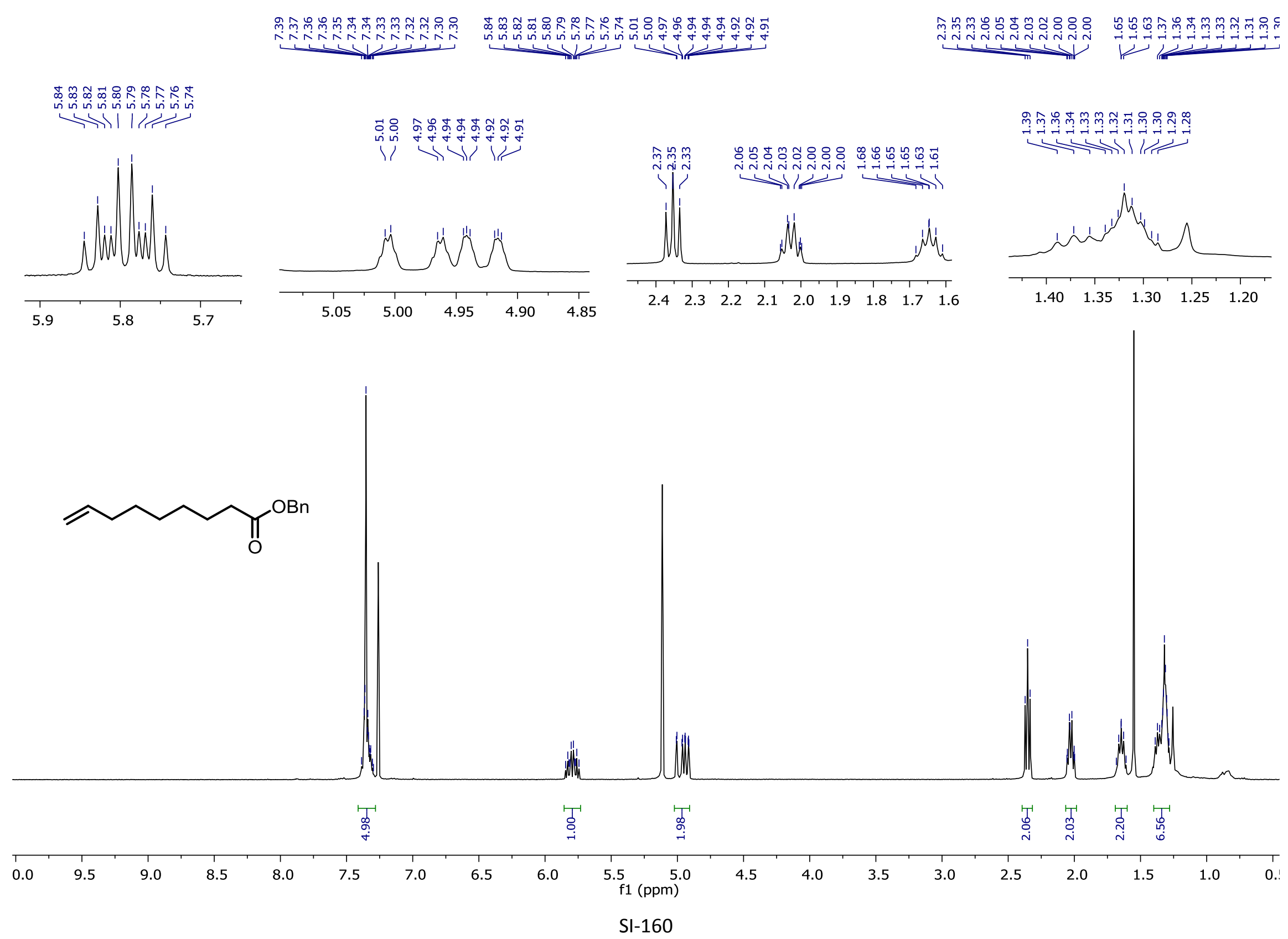


${ }^{13} \mathrm{C}-\mathrm{NMR}\left(101 \mathrm{MHz}, \mathrm{CDCl}_{3}\right)$ for compound 4ab'

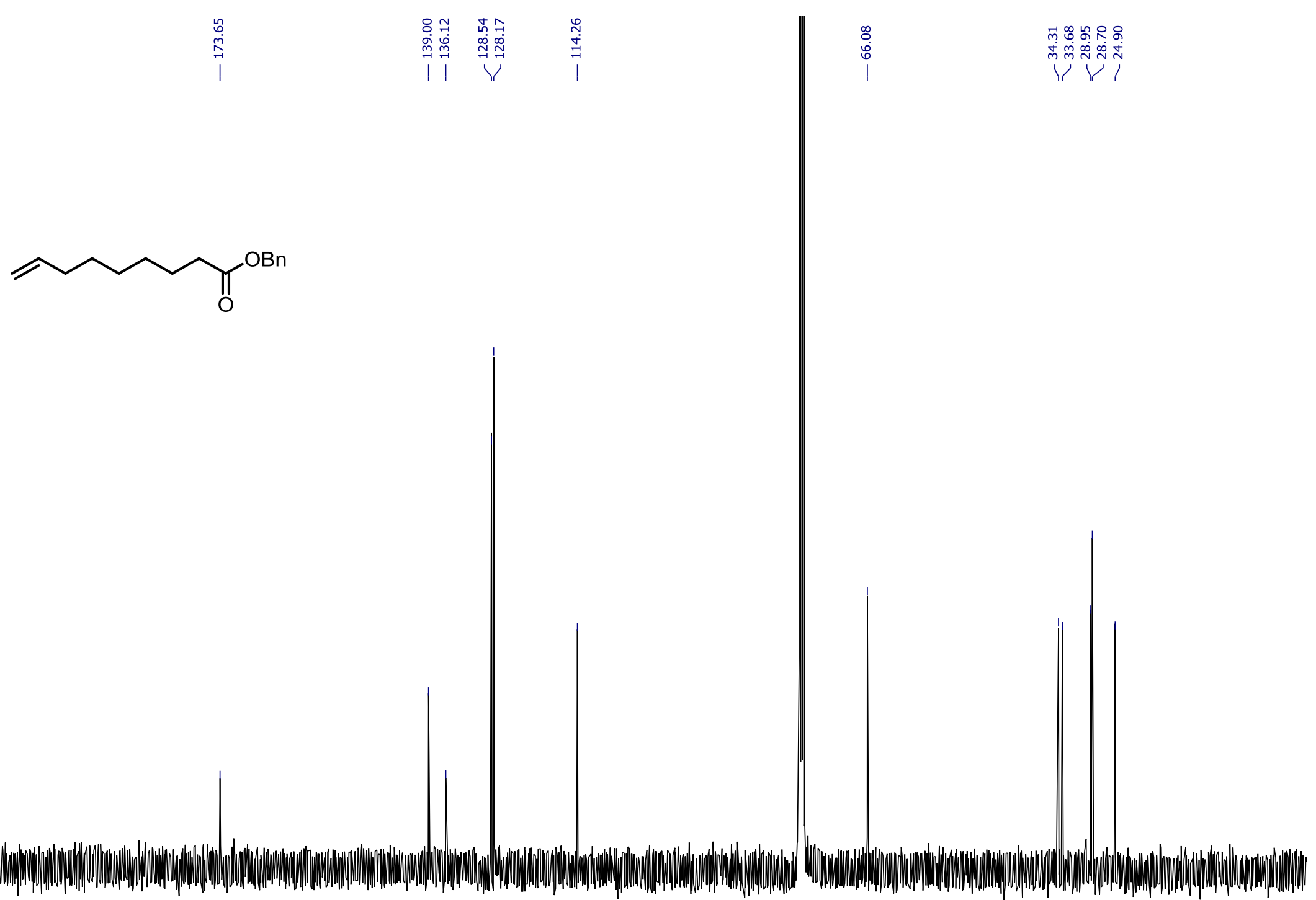

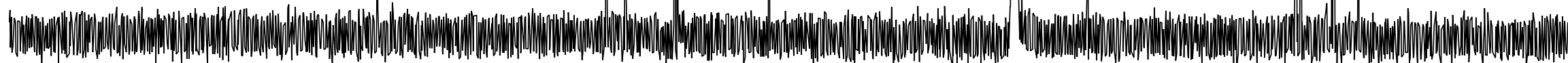

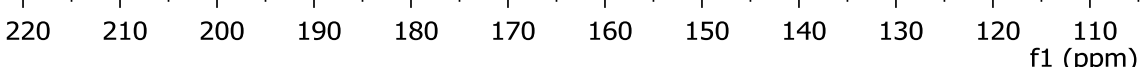

SI-161 


\section{${ }^{1} \mathrm{H}-\mathrm{NMR}\left(400 \mathrm{MHz}, \mathrm{CDCl}_{3}\right)$ for compound $4 a-\boldsymbol{d}_{1}$}

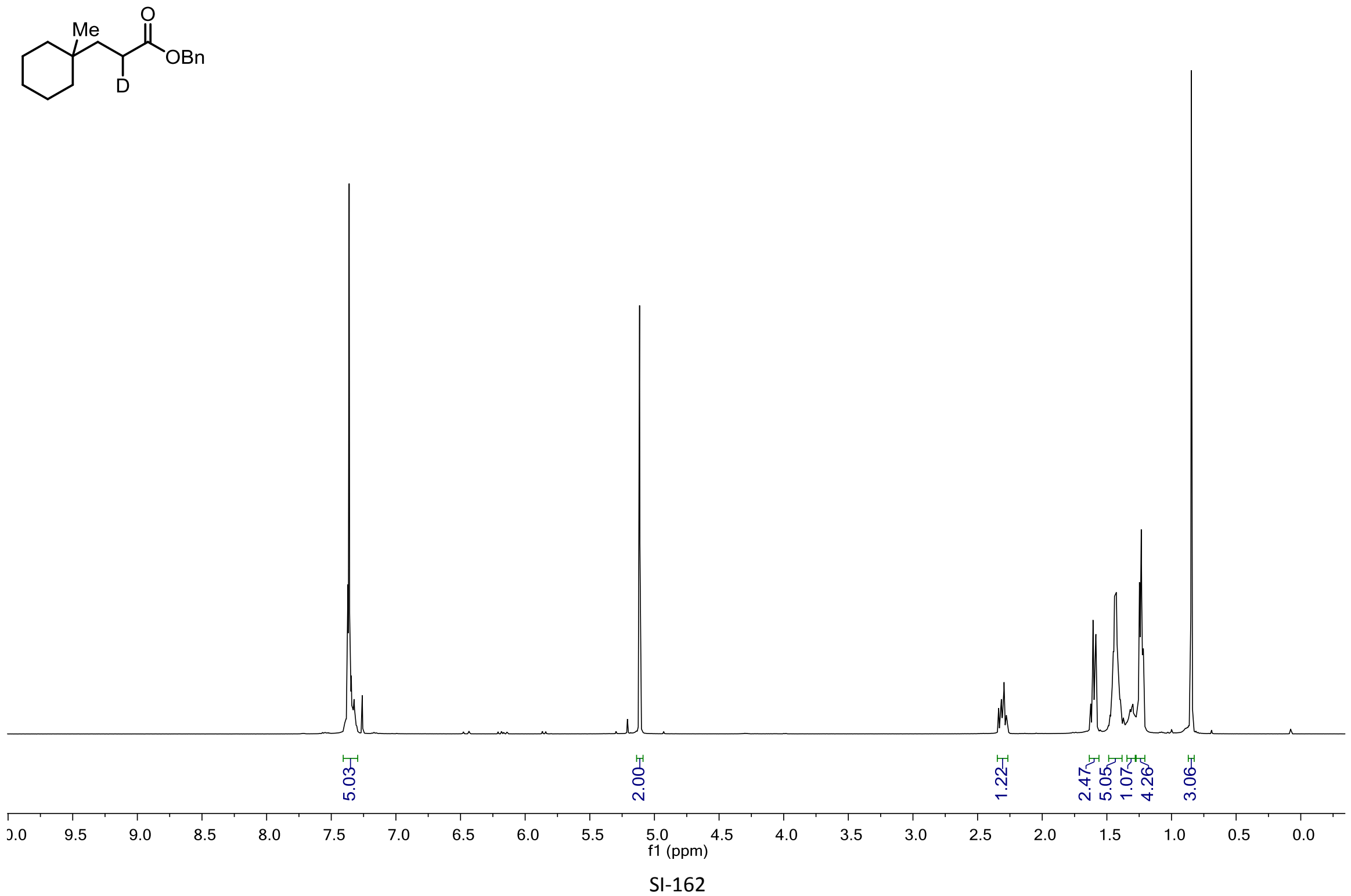


${ }^{13} \mathrm{C}-\mathrm{NMR}\left(101 \mathrm{MHz}, \mathrm{CDCl}\right.$ ) for compound $\mathbf{4 a - d _ { 1 }}$

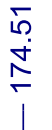
듀유ำ

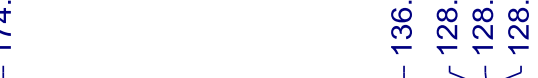

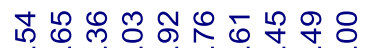
ஸे山<smiles>[2H]C(CC1(C)CCCCC1)C(=O)OCc1ccccc1</smiles>

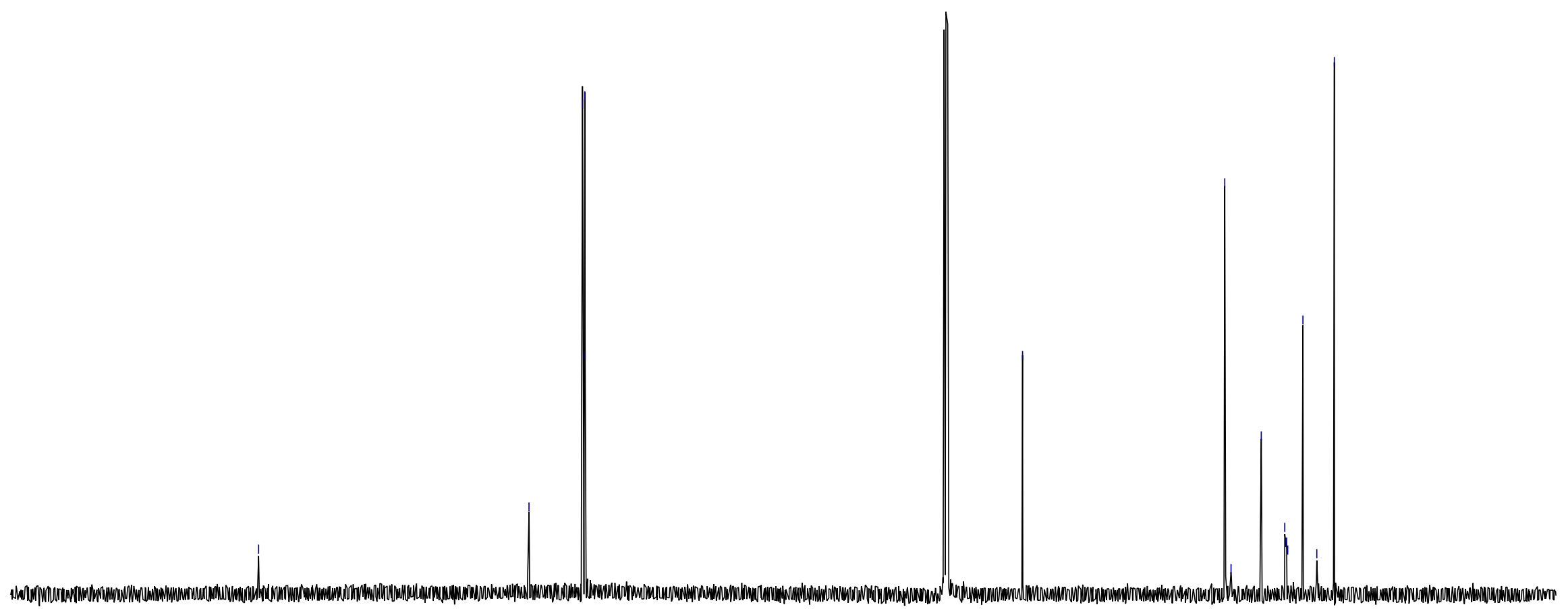

